\title{
Steady-State Flammable Gas Release Rate Calculation and Lower Flammability Level Evaluation for Hanford Tank Waste
}

\author{
J. E. Meacham \\ Washington River Protection Solutions \\ Richland, WA 99352 \\ U.S. Department of Energy Contract DE-AC27-08RV14800 \\ EDT/ECN: $\quad 721735 \mathrm{R} 13 \quad$ UC: $\mathrm{N} / \mathrm{A}$ \\ Cost Center: $\quad$ Charge Code: \\ B\&R Code: N/A Total Pages: 98
}

Key Words: Double-shell tank, single-shell tank, DST, SST, flammability, time to lower flammability limit, TTLFL, hydrogen and headspace.

Abstract: This report assesses the steady state flammability level under off normal ventilation conditions in the tank headspace for 28 double-shell tanks (DST) and 149 single shell-tanks (SST) at the Hanford Site. Flammability was calculated using estimated gas release rates, Le Chatelier's rule, and lower flammability limits of fuels in an air mixture. This revision updates the hydrogen generation rate input data for all 177 tanks using waste composition information from the Best Basis Inventory Detail Report (data effective as of August 4, 2008). Assuming only barometric breathing, the shortest time to reach $25 \%$ of the lower flammability limit is 11 days for DSTs (i.e., tank 241-AZ-101) and 36 days for SSTs (i.e., tank 241-B-203). Assuming zero ventilation, the shortest time to reach $25 \%$ of the lower flammability limit is 10 days for DSTs (i.e., tank 241-AZ-101) and 34 days for SSTs (i.e., tank 241-B-203).

TRADEMARK DISCLAIMER. Reference herein to any specific commercial product, process, or service by trade name, trademark, manufacturer, or otherwise, does not necessarily constitute or imply its endorsement, recommendation, or favoring by the United States Government or any agency thereof or its contractors or subcontractors.
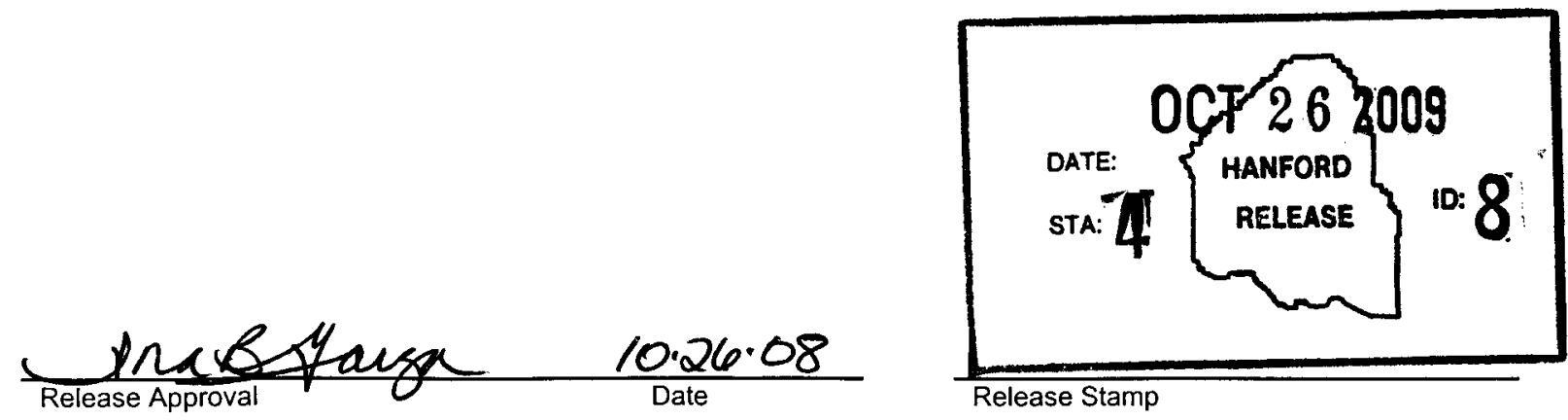

Approved For Public Release 


\begin{tabular}{|c|c|c|c|}
\hline \multicolumn{2}{|r|}{$\begin{array}{l}\text { Tank Farm Contractor (TFC) } \\
\text { RECORD OF REVISION }\end{array}$} & $\begin{array}{l}\text { (1) Document Number: } \\
\text { RPP-5926 }\end{array}$ & Page 1 \\
\hline \multicolumn{4}{|c|}{$\begin{array}{l}\text { (2) Title: } \\
\text { Steady-State Flammable Gas Release Rate Calculation and Lower Flammability Level Evaluation for Hanford Tank Waste }\end{array}$} \\
\hline \multicolumn{4}{|c|}{ Change Control Record } \\
\hline \multirow{2}{*}{$\begin{array}{c}\text { (3) } \\
\text { Revision } \\
\end{array}$} & \multirow{2}{*}{ (4) Description of Change - Replace, Add, and Delete Pages } & \multicolumn{2}{|c|}{ Authorized for Release } \\
\hline & & (5) Resp. Engr. (print/sign/date) & (6) Resp. Mgr. (print/sign/date) \\
\hline 0 & Original Release via EDT 628264 & $\begin{array}{l}\text { T. A. Hu } \\
4 / 27 / 2000\end{array}$ & $\begin{array}{l}\text { K. M. Hodgson } \\
4 / 27 / 2000\end{array}$ \\
\hline 1 & $\begin{array}{l}\text { Total revision to address comments resulting from DOE- } \\
\text { ORP review (ECN 664031) }\end{array}$ & $\begin{array}{l}\text { T. A. Hu } \\
2 / 23 / 2001\end{array}$ & $\begin{array}{l}\text { J. S. Garfield } \\
2 / 23 / 2001\end{array}$ \\
\hline 2 & $\begin{array}{l}\text { Total revision to update the hydrogen rates and } \\
\text { flammability to Nov. } 2001 \text { BBI data. (ECN 665039) }\end{array}$ & $\begin{array}{l}\text { T. A. Hu } \\
6 / 30 / 2002\end{array}$ & $\begin{array}{l}\text { N. W. Kirch } \\
6 / 30 / 2002\end{array}$ \\
\hline $2 \mathrm{~A}$ & $\begin{array}{l}\text { Add Appendix I, flammability evaluation of } 5 \text { DSTs with } \\
\text { highest LFL. (ECN 720321) }\end{array}$ & $\begin{array}{l}\text { T. A. Hu } \\
3 / 20 / 2003\end{array}$ & $\begin{array}{l}\text { N. W. Kirch } \\
3 / 20 / 2003\end{array}$ \\
\hline 3 & $\begin{array}{l}\text { Total revision to update the hydrogen rates and } \\
\text { flammability to July } 2003 \mathrm{BBI} \text { data. }\end{array}$ & $\begin{array}{l}\text { T. A. Hu } \\
9 / 30 / 2003\end{array}$ & $\begin{array}{l}\text { N. W. Kirch } \\
9 / 30 / 2003\end{array}$ \\
\hline $3 \mathrm{~A}$ & $\begin{array}{l}\text { Add Appendix J, flammability evaluation of vacuum } \\
\text { retrieval project of } 241 \text {-C Tank Farm 200-Series }\end{array}$ & $\begin{array}{l}\text { T. A. Hu } \\
11 / 19 / 2003\end{array}$ & $\begin{array}{l}\text { T. M. Horner } \\
11 / 19 / 2003\end{array}$ \\
\hline $3 \mathrm{~B}$ & $\begin{array}{l}\text { Add Appendix K, flammability evaluation to raise DST } \\
\text { operation waste level to } 460 \text { inch. }\end{array}$ & $\begin{array}{l}\text { T. A. Hu } \\
12 / 23 / 2003\end{array}$ & $\begin{array}{l}\text { T. M. Horner } \\
12 / 23 / 2003\end{array}$ \\
\hline $3 \mathrm{C}$ & $\begin{array}{l}\text { Add Appendix L, flammability evaluation of retrieval } \\
\text { project on } 241-\mathrm{C}-103 \text { and } 241-\mathrm{C}-105\end{array}$ & $\begin{array}{l}\text { T. A. Hu } \\
1 / 22 / 2004\end{array}$ & $\begin{array}{l}\text { T. M. Horner } \\
1 / 22 / 2004\end{array}$ \\
\hline $3 \mathrm{D}$ & $\begin{array}{l}\text { Add Appendix M, flammability evaluation of 241-AY- } \\
102 \text { to support receiving condensate }\end{array}$ & $\begin{array}{l}\text { T. A. Hu } \\
4 / 20 / 2004\end{array}$ & $\begin{array}{l}\text { T. M. Horner } \\
4 / 20 / 2004\end{array}$ \\
\hline $3 \mathrm{E}$ & $\begin{array}{l}\text { Update Appendix K, (Title, see Rev 3B) ECN-721735- } \\
\text { R1 }\end{array}$ & $\begin{array}{l}\text { T. A. Hu } \\
6 / 2 / 2004\end{array}$ & $\begin{array}{l}\text { T. M. Horner } \\
6 / 2 / 2004\end{array}$ \\
\hline 4 & $\begin{array}{l}\text { Complete revision of document as annual update per } \\
\text { ECN-721735-R2 }\end{array}$ & $\begin{array}{l}\text { T. A. Hu } \\
10 / 27 / 2004\end{array}$ & $\begin{array}{l}\text { T. M. Horner } \\
10 / 27 / 2004\end{array}$ \\
\hline $4 \mathrm{~A}$ & $\begin{array}{l}\text { Address ORP comments on Rev } 4 \text { and correct editorial } \\
\text { errors per ECN-721735-R3 }\end{array}$ & $\begin{array}{l}\text { T. A. } \mathrm{Hu} \\
2 / 17 / 2005\end{array}$ & $\begin{array}{l}\text { T. M. Horner } \\
2 / 17 / 2005\end{array}$ \\
\hline 4B & $\begin{array}{l}\text { Add Appendix I, flammability evaluation on raising the } \\
\text { waste level to } 460 \text { inch for DSTs per ECN-721735-R4 }\end{array}$ & $\begin{array}{l}\text { T. A. Hu } \\
4 / 27 / 2005\end{array}$ & $\begin{array}{l}\text { T. M. Horner } \\
4 / 27 / 2005\end{array}$ \\
\hline $4 \mathrm{C}$ & Delete the content of Appendix I per ECN-721735-R5 & $\begin{array}{l}\text { T. A. Hu } \\
6 / 15 / 2005\end{array}$ & $\begin{array}{l}\text { T. M. Horner } \\
6 / 15 / 2005\end{array}$ \\
\hline $4 \mathrm{D}$ & $\begin{array}{l}\text { Add Appendix I content with the flammability evaluation } \\
\text { on the uncovered solids for DSTs per ECN-721735-6 }\end{array}$ & $\begin{array}{l}\text { T. A. Hu } \\
7 / 28 / 2005\end{array}$ & $\begin{array}{l}\text { T. M. Horner } \\
7 / 27 / 2005\end{array}$ \\
\hline $4 \mathrm{E}$ & $\begin{array}{l}\text { Add Appendix J content with the flammability } \\
\text { evaluation on tank } 241-\mathrm{C}-103 \text { retrieval per ECN-721735- } \\
\text { R7 }\end{array}$ & $\begin{array}{l}\text { T. A. Hu } \\
9 / 12 / 2005\end{array}$ & $\begin{array}{l}\text { T. M. Horner } \\
9 / 12 / 2005\end{array}$ \\
\hline 5 & $\begin{array}{l}\text { Complete revision of document as annual update per } \\
\text { ECN-721735-R8 }\end{array}$ & $\begin{array}{l}\text { T. A. Hu } \\
10 / 27 / 2005\end{array}$ & $\begin{array}{l}\text { M. A. Knight } \\
10 / 27 / 2005\end{array}$ \\
\hline 6 & $\begin{array}{l}\text { Complete revision of document as annual update per } \\
\text { ECN-721735-R9 }\end{array}$ & $\begin{array}{l}\text { T. A. Hu } \\
10 / 27 / 2006\end{array}$ & $\begin{array}{l}\text { N. W. Kirch } \\
10 / 27 / 2006\end{array}$ \\
\hline 7 & $\begin{array}{l}\text { Complete revision of document as annual update per } \\
\text { ECN-721735-R10 }\end{array}$ & $\begin{array}{l}\text { T. A. Hu } \\
10 / 25 / 2007\end{array}$ & $\begin{array}{l}\text { N. W. Kirch } \\
10 / 25 / 2007\end{array}$ \\
\hline 8 & $\begin{array}{l}\text { Complete revision of document as annual update per } \\
\text { ECN-721735-R11 }\end{array}$ & $\begin{array}{l}\text { J. E. Meacham } \\
11 / 17 / 08\end{array}$ & $\begin{array}{l}\text { N. W. Kirch } \\
11 / 17 / 08\end{array}$ \\
\hline
\end{tabular}




\begin{tabular}{|c|c|c|c|}
\hline \multicolumn{2}{|r|}{$\begin{array}{l}\text { Tank Farm Contractor (TFC) } \\
\text { RECORD OF REVISION }\end{array}$} & $\begin{array}{l}\text { (1) Document Number: } \\
\text { RPP-5926 }\end{array}$ & Page 2 \\
\hline \multicolumn{4}{|c|}{ Change Control Record } \\
\hline \multirow{2}{*}{$\begin{array}{c}(3) \\
\text { Revision }\end{array}$} & \multirow{2}{*}{ (4) Description of Change - Replace, Add, and Delete Pages } & \multicolumn{2}{|c|}{ Authorized for Release } \\
\hline & & (5) Resp. Engr. (print/sign/date) & (6) Resp. Mgr. (print/sign/date) \\
\hline $8 \mathrm{~A}$ & $\begin{array}{l}\text { Add discussion of SST temperature trends (App. E) per } \\
\text { ECN-721735-R12 }\end{array}$ & $\begin{array}{l}\text { J. M. Conner } \\
08 / 19 / 2009\end{array}$ & $\begin{array}{l}\text { N. W. Kirch } \\
08 / 20 / 2009\end{array}$ \\
\hline R5 9 & $\begin{array}{l}\text { Revision of document as annual update per ECN- } \\
721735-\mathrm{R} 13\end{array}$ & J. E. Meacham /L/ha & N. W. Kirch 1 WKKul \\
\hline
\end{tabular}


RPP-5926

Revision 9

\section{Steady-State Flammable Gas \\ Release Rate Calculation and Lower \\ Flammability Level Evaluation for \\ Hanford Tank Waste}

J. E. Meacham

Washington River Protection Solutions

Date Published

October 2009

Prepared for the U.S. Department of Energy

Assistant Secretary for Environmental Management

Contractor for the U.S. Department of Energy

Office of River Protection under Contract DE-AC27-08RV14800

washington river

protectionsolutions

Washington River Protection Solutions LLC

P.O. Box 850

Richland, Washington 


\section{EXECUTIVE SUMMARY}

Flammable gases such as hydrogen, ammonia, and methane are observed in the tank headspace of the Hanford Site high-level waste tanks. This report assesses the steady-state flammability level under off-normal ventilation conditions in the tank headspace for 28 double-shell tanks (DST) and 149 single-shell tanks (SST) at the Hanford Site. Flammability was calculated using estimated gas release rates, Le Chatelier's rule, and lower flammability limits of fuels in an air mixture. A time-dependent equation of gas concentration, which is a function of the gas release and ventilation rates in the headspace, was developed. With this dynamic model, the time required to reach the specified flammability level at a given ventilation rate was calculated. Hydrogen generation rates were calculated using the empirical rate equation model provided in Empirical Rate Equation Model and Rate Calculations of Hydrogen Generation for Hanford Tank Waste, HNF-3851. The off-normal ventilation rate is assumed to be barometric breathing only or zero ventilation.

This revision updates the hydrogen generation rate input data for all 177 tanks using waste composition information from the Best Basis Inventory Detail Report in the Tank Waste Information Network System (effective date July 6, 2009), and the waste temperature data in the Surveillance Analysis Computer System. All DST waste temperatures were increased $5{ }^{\circ} \mathrm{C}$ to address potential small temperature increases. A 10,000 gallon water addition was also assumed for all DSTs and 100-series SSTs to address operations than involve small water additions. The smaller 200-series SSTs were assumed to have 1,000 gallon water additions. Gas diffusion through the concrete dome is included in the mass balance equations for SSTs under the zero ventilation condition.

Assuming only barometric breathing, the shortest time to reach $25 \%$ of the lower flammability limit is 11 days for DSTs (i.e., tank 241-AZ-101) and 36 days for SSTs (i.e., tank 241-B-203). Assuming zero ventilation, the shortest time to reach $25 \%$ of the lower flammability limit is 10 days for DSTs (i.e., tank 241-AZ-101) and 34 days for SSTs (i.e., tank 241-B-203). 


\section{CONTENTS}

1.0 PURPOSE

2.0

3.0

BACKGROU

ASSUMPTIONS..

4.0

METHODOLOGY

4.1 HYDROGEN GENERATION SEMI-EMPIRICAL RATE EQUATIONS............3

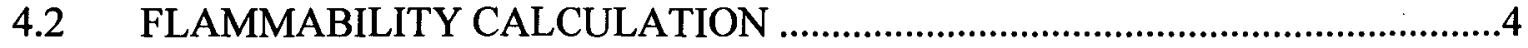

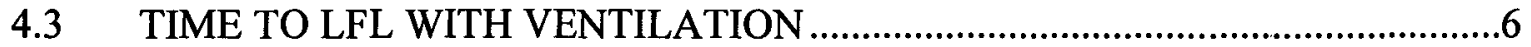

4.4 TIME TO LFL WITH ZERO VENTILATION..................................................8

4.5 MINIMUM VENTILATION RATE TO MAINTAIN HEADSPACE BELOW 25 OR 100 PERCENT OF THE LFL

5.0 INPUT DATA.

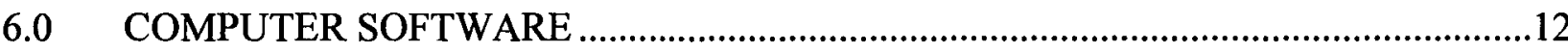

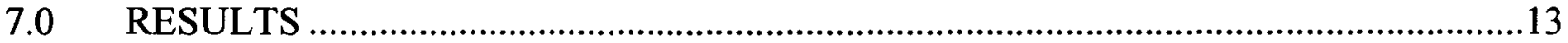

7.1 DOUBLE-SHELL TANK RESULTS ...........................................................13

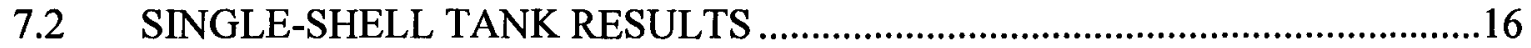

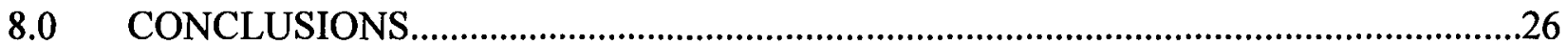

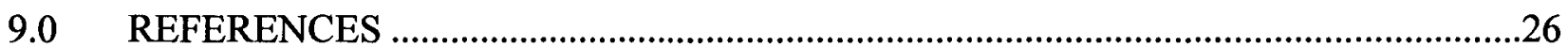

\section{APPENDICES}

A INPUT DATA FOR TIME TO THE LOWER FLAMMABILITY LIMIT CALCULATIONS

B CALCULATIONS OF THE GAS DIFFUSION COEFFICIENTS FOR THE CONCRETE DOME OF SINGLE-SHELL TANKS

C AMMONIA CONTRIBUTION TO FLAMMABILITY

D EVALUATION OF SST TEMPERATURE TRENDS

D-1

E PEER REVIEW CHECKLIST 


\section{LIST OF TABLES}

Table 5-1. Curie to Watt Conversion Factors

Table 7-1. Double-Shell Tank Steady-State Flammable Gas Concentration and Time to Lower Flammability Limit Under Barometric Breathing

Table 7-2. Double-Shell Tank Time to Lower Flammability Limit Under Zero Ventilation

Table 7-3. Double-Shell Tank Hydrogen Generation Rate and Minimum Ventilation Rate Required to Maintain Headspace Flammable Gas Concentration Below Specified Level.

Table 7-4. Single-Shell Tank Steady-State Flammable Gas Concentration and Time to Lower Flammability Limit Under Barometric Breathing.

Table 7-5. Single-Shell Tank Time to Lower Flammability Limit Under Zero Ventilation

Table 7-6. Single-Shell Tank Hydrogen Generation Rate and Minimum Ventilation Rate Required to Maintain Headspace Flammable Gas Concentration Below Specified Level. 


\section{LIST OF TERMS}

BBI

DST

GRE

HEPA

HGR

LFL

SACS

SST

TCD

TOC

TTLFL

TWINS best-basis inventory

double-shell tank

gas release event

high-efficiency particulate air (filter)

hydrogen generation rate

lower flammability limit

Surveillance Analysis Computer System

single-shell tank

Tank Characterization Database

total organic carbon

time to lower flammability limit

Tank Waste Information Network System 


\subsection{PURPOSE}

This report evaluates steady-state flammability levels in tank headspaces under off-normal ventilation conditions for 177 underground nuclear waste storage tanks at the Hanford Site.

\subsection{BACKGROUND}

This document provides the technical basis for establishing flammable gas controls. The focus of this report is the loss of ventilation transient such that technical safety requirement surveillance frequencies and action completion times can be established. This report also provides the following:

- Estimates of the time to reach $25 \%$ of the lower flammability limit (LFL) and $100 \%$ of the LFL following the loss of normal ventilation in actively and passively ventilated double-shell tanks (DST) and single-shell tanks (SST)

- Estimates of the minimum ventilation rate required to maintain less than $25 \%$ and $100 \%$ of the LFL

Steady-state implies that no active operational changes and no gas release events (defined as a 500 -ppm rise in hydrogen concentration followed by exponential decay of the released flammable gas) are occurring. 


\subsection{ASSUMPTIONS}

Barometric breathing is taken to be the yearly average rate and variations brought about by seasonal effects over short durations are neglected.

Methane generation is assumed to be $10 \%$ of the model-calculated hydrogen generation from thermolysis and radiolysis. This is a reasonable estimate because, for all tanks, methane is less than $10 \%$ of the hydrogen in the generated gas as given in the gas generation study (PNNL-12181) and retained gas sampler results (PNNL-13000).

Peak waste temperatures for hydrogen generation in DSTs are assumed to be $5{ }^{\circ} \mathrm{C}$ higher than actually measured for the year to address potential small temperature increases.

Calculations include a 10,000 gallon water addition (1,000 gallon water addition to 200-series SSTs) to address operations that involve small water additions.

Hypothetical water additions are assumed to not significantly influence the overall tank hydrogen generation efficiency coefficient from corrosion $\left(\mathrm{E}_{\mathrm{H}_{2}}\right)$.

When the solids and supernatant layers have different hydrogen generation efficiency coefficients from corrosion, the higher of the two (i.e., 0.5 ) is conservatively selected to represent the tank. This assumption simplifies the calculations and does not significantly affect results.

Tank headspaces are assumed to start at $2 \%$ of the LFL to account for ammonia contribution to flammability (see Appendix C).

Hydrogen and methane starting concentrations are assumed negligible.

Beta and gamma heat loads are assumed to be comprised of ${ }^{90} \mathrm{Sr}$ and ${ }^{137} \mathrm{Cs}$. Alpha heat load was assumed to be comprised of ${ }^{241} \mathrm{Am},{ }^{240} \mathrm{Pu},{ }^{239} \mathrm{Pu}$, and ${ }^{238} \mathrm{Pu}$. These are reasonable simplifying assumptions given that these radionuclides dominate their respective heat loads.

Nitrous oxide concentration is assumed to be below $8 \%$ and therefore does not decrease the LFL for hydrogen, methane, or ammonia (see discussion in Section 4.2). 


\subsection{METHODOLOGY}

The time to the lower flammability limit (TTLFL) is a matter of determining the rate of flammable gas accumulation in the tank headspace and then calculating the time necessary to reach flammability. The first step is to calculate the hydrogen generation (Section 4.1) and the gas mixture flammability (Section 4.2). Flammability at any given time is a balance between gas generation and the headspace ventilation rate (Sections 4.3 through 4.5 ).

\subsection{HYDROGEN GENERATION SEMI-EMPIRICAL RATE EQUATIONS}

A comprehensive hydrogen generation rate (HGR) model was developed and validated based on a large body of tank waste data (HNF-3851). The model constructed a set of semi-empirical rate equations to simulate the gas generation mechanisms of thermal chemical reactions, radiolysis of water and organic components, and corrosion processes. The rate equations are formulated as a function of physical and chemical properties of tank waste and are able to estimate the HGR of waste under current conditions, including the rates for newly mixed waste with known properties.

The empirical rate equation for hydrogen generation in Hanford Site waste contains contributions from thermal reactions, $\mathrm{HGR}_{\mathrm{thm}}$, radiolysis of water and organic, $\mathrm{HGR}_{\mathrm{rad}}$, and corrosion, $\mathrm{HGR}_{\text {corr }}$. This rate equation is a function of waste composition (TOC, $\mathrm{Al}^{+3}, \mathrm{Na}^{+}, \mathrm{NO}_{2}^{-}, \mathrm{NO}_{3}^{-}$, and $\left.\mathrm{OH}^{-}\right)$, radiation dose, temperature, liquid fraction, and tank wetted area. Both the thermal and organic radiolysis rates follow Arrhenius behavior with a derived activation energy. The equation for HGR in the units of moles per kilogram per day can be summarized as follows (Equation 4-1):

Where:

$$
\mathrm{HGR}=\mathrm{HGR}_{\mathrm{thm}}+\mathrm{HGR}_{\mathrm{rad}}+\mathrm{HGR}_{\mathrm{corr}}
$$

$$
\begin{aligned}
& \mathrm{HGR}_{\text {thm }}=\mathrm{a}_{\mathrm{thm}} \times\left(\mathrm{r}_{\mathrm{f}} \times[\mathrm{TOC}]\right) \times\left[\mathrm{Al}^{+3}\right]^{0.4} \times \mathrm{L}_{\mathrm{f}} \times \exp ^{\left(-\mathrm{E}_{\mathrm{thm}} / \mathrm{RT}\right)}
\end{aligned}
$$

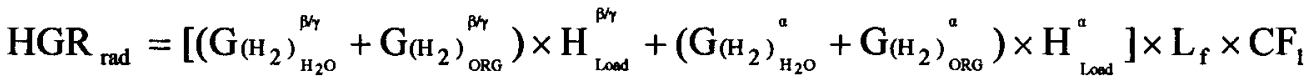

$$
\begin{aligned}
& \mathrm{HGR}_{\text {corr }}=\mathrm{R}_{\text {corr }} \times \mathrm{E}_{\mathrm{H}_{2}} \times \mathrm{A}_{\text {wetted }} / \mathrm{M}_{\text {tank }} \times \mathrm{CF}_{2}
\end{aligned}
$$

with:

$$
\begin{aligned}
& \mathrm{G}_{\left(\mathrm{H}_{2}\right)_{\mathrm{ORG}}{ }^{\beta / \gamma}}=\mathrm{a}_{\mathrm{rad}} \times \exp ^{\left(-\mathrm{E}_{\mathrm{rad}} / \mathrm{RT}\right)} \times\left(\mathrm{r}_{\mathrm{f}} \times[\mathrm{TOC}]\right) \\
& \mathrm{G}_{\left(\mathrm{H}_{2}\right)_{\mathrm{H}_{2} \mathrm{O}}^{\mathrm{B} / \mathrm{\gamma}}}=\frac{0.32}{1+2.4\left[\mathrm{NO}_{3}^{-}\right]+0.62\left[\mathrm{NO}_{2}^{-}\right]+0.31\left[\mathrm{Na}^{+}\right]_{\mathrm{ex}}^{2}}+\frac{0.13}{1+139\left[\mathrm{NO}_{3}^{-}\right]+54\left[\mathrm{NO}_{2}^{-}\right]}
\end{aligned}
$$

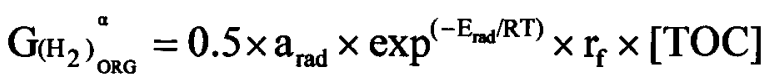

$$
\begin{aligned}
& \mathrm{G}_{\left(\mathrm{H}_{2}\right)_{\mathrm{H}_{2} \mathrm{O}}{ }^{\alpha}}=\frac{1.05}{1+2.4\left[\mathrm{NO}_{3}^{-}\right]+0.63\left[\mathrm{NO}_{2}^{-}\right]}+\frac{0.35}{1+3900\left[\mathrm{NO}_{3}^{-}\right]+1400\left[\mathrm{NO}_{2}^{-}\right]}
\end{aligned}
$$

Where:

$$
\begin{aligned}
\mathrm{E}_{\mathrm{thm}} & =89.6 \mathrm{~kJ} / \mathrm{mole} \text {, the activation energy for the thermal reaction } \\
\mathrm{a}_{\mathrm{thm}} & =3.94 \mathrm{E}+09 \text { moles } / \mathrm{kg} \text {-day, pre-exponential factor of the thermal rate }
\end{aligned}
$$


$\mathrm{E}_{\mathrm{rad}}=48.8 \mathrm{~kJ} / \mathrm{mole}$, activation energy in organic radiolysis, $\mathrm{G}$

$a_{\text {rad }}=1.11 \mathrm{E}+07 \mathrm{H}_{2} / 100 \mathrm{eV}$, the pre-exponential term in organic radiolysis, $\mathrm{G}$

$r_{\mathrm{f}}=0.6$ for DSTs and 0.3 for SSTs (dimensionless), the total organic carbon reactivity coefficient. The 0.3 for SSTs is an average value from the tanks tested. If tank has a high fraction of energetic organic compounds, $r_{f}$ can be adjusted to as high as 0.6.

$\mathrm{R}=8.314 \mathrm{~J} / \mathrm{mole}-\mathrm{K}$, gas constant

$\mathrm{R}_{\text {corr }}=6.0 \mathrm{E}-08$ for DSTs and 2.4 E-07 for SSTs $\left(\mathrm{ft}^{3} / \mathrm{min}-\mathrm{ft}^{2}\right)$, corrosion coefficient

$\mathrm{E}_{\mathrm{H}_{2}}=$ hydrogen generation efficiency coefficient from corrosion is 0.20 if $\left[\mathrm{NO}_{3}{ }^{-}\right],\left[\mathrm{NO}_{2}{ }^{-}\right]$, and $\left[\mathrm{OH}^{-}\right]>0.1$, otherwise it is 0.50 .

$[\mathrm{TOC}]=$ total organic carbon concentration in the liquid waste, wt\% Note: Insoluble energetic organic compounds (excluding oxalate) in the solid layer should be considered case by case when data is available

$\left[\mathrm{Al}^{+3}\right]=$ aluminum ion concentration in liquid waste, $\mathrm{wt} \%$

$\left[\mathrm{NO}_{3}{ }^{-}\right]=$nitrate ion concentration in the liquid waste, moles $/ \mathrm{L}$

$\left[\mathrm{NO}_{2}{ }^{-}\right]=$nitrite ion concentration in the liquid waste, moles $/ \mathrm{L}$

$\left[\mathrm{Na}^{+}\right]_{\mathrm{ex}}=$ concentration of sodium minus nitrate and nitrite concentration in liquid waste, moles/L

$\mathrm{H}_{\text {Lood }}^{\mathrm{B} / \mathrm{r}}=$ total heat load of the tank from beta/gamma, watt $/ \mathrm{kg}$

$\mathbf{H}_{\text {Lood }}^{\alpha}=$ total heat load of the tank from total alpha, watt $/ \mathrm{kg}$

$\mathrm{L}_{\mathrm{f}}=$ liquid weight fraction in the waste, dimensionless

$\mathrm{T}=$ temperature of waste, $\mathrm{K}$

$\mathrm{A}_{\text {wetted }}=$ area of steel exposed to moisture-containing waste, $\mathrm{ft}^{2}$

$\mathrm{M}_{\text {tank }}=$ total waste mass in the tank, $\mathrm{kg}$

$\mathrm{CF}_{1}=$ conversion factor from $\mathrm{H}_{2}$-watt $/ 100 \mathrm{eV}-\mathrm{kg}$ to moles $/ \mathrm{kg}$-day

$\mathrm{CF}_{2}=$ conversion factor from $\mathrm{m}^{3} / \mathrm{kg}-\mathrm{min}$ to moles $/ \mathrm{kg}$-day

In general, hydrogen generation from chemical reactions follows Arrhenius behavior and the rate from radiolysis is proportional to the radiation dose. Hydrogen generation from radiation effects in tank waste samples have been observed to be temperature dependent as provided in PNNL-12181. The analysis of all available gas generation data provided in HNF-3851 shows that this temperature-dependent reaction follows Arrhenius behavior, which may result from a multi-step degradation of organic compounds initiated by radiolysis, followed by thermal reactions. The water radiolysis rate is temperature independent, with the value reduced by scavenging effects.

\subsection{FLAMMABILITY CALCULATION}

The National Fire Protection Agency codes require that operations be terminated when the concentration of flammable gases exceeds $25 \%$ of the LFL. Based on the empirical rule developed by Le Chatelier in the late 19th century, the LFL of mixtures of multiple flammable gases in air can be determined. Le Chatelier's rule (Limits of Flammability of Gases and Vapors, [Coward and Jones 1952]) can be written in terms of the LFL concentration of the fuel mixture, $\mathrm{LFL}_{\mathrm{m}}$, as follows: 


$$
\mathrm{LFL}_{\mathrm{m}}=\frac{100}{\sum_{i}^{n}\left(\frac{\mathrm{C}_{\mathrm{i}}}{\mathrm{LFL}_{\mathrm{i}}}\right)}
$$

where:

$\mathrm{C}_{\mathrm{i}} \quad=$ volume percent of fuel gas $i$ in the fuel gas mixture

$\mathrm{LFL}_{\mathrm{i}}=$ volume percent of fuel gas $i$ at its LFL in air alone.

In the tank headspace, hydrogen gas, ammonia, and methane are the flammable gases of concern. For tank waste conditions, the volume percents of the flammable gases vary over time depending on flammable gas release rates into the tank headspace, and thus, Equation 4-2 cannot be directly applied. WHC-SD-WM-ES-219 showed experimentally that the total flammability level $\left(\mathrm{LFL}_{\mathrm{f}}\right)$ of a hydrogen, ammonia, and methane mixture in an air-nitrous oxide atmosphere can be computed using Le Chatelier's principle (Equation 4-3).

where:

$$
\mathrm{LFL}_{\mathrm{f}}=\frac{\left[\mathrm{H}_{2}\right]}{\mathrm{LFL}_{\mathrm{H}_{2}}}+\frac{\left[\mathrm{NH}_{3}\right]}{\mathrm{LFL}_{\mathrm{NH}_{3}}}+\frac{\left[\mathrm{CH}_{4}\right]}{\mathrm{LFL}_{\mathrm{CH}_{4}}}
$$

$\left[\mathrm{H}_{2}\right]=$ volume percent of hydrogen in the tank headspace $\left[\mathrm{NH}_{3}\right]=$ volume percent of ammonia in the tank headspace $\left[\mathrm{CH}_{4}\right]=$ volume percent of methane in the tank headspace $\mathrm{LFL}_{\mathrm{H}_{2}}=$ volume percent of hydrogen at its LFL in air alone $\mathrm{LFL}_{\mathrm{NH}_{3}}=$ volume percent of ammonia at its LFL in air alone $\mathrm{LFL}_{\mathrm{CH}_{4}}=$ volume percent of methane at its LFL in air alone.

For simplicity, flammable gases with concentrations lower than that of methane are omitted. The flammability of the individual gases in air is fairly well characterized. The upward propagation LFL for hydrogen, ammonia and methane is 4,15 , and 5\%, respectively. In addition to the flammable gases included in Equation 4-3, nitrous oxide was observed in the tank headspace. Nitrous oxide plays the role of oxidizer in the mixture and has the potential to decrease the amount of fuel required to reach the LFL. The effect of the multiple oxidizers (e.g., $\mathrm{N}_{2} \mathrm{O}$ and $\mathrm{O}_{2}$ ) was not addressed in Equation 4-3.

Pfahl and Shepherd (1997) from the California Institute of Technology conducted a series of flammability studies on gas mixtures generated from Hanford Site tank waste. The studies show that the LFLs of individual fuels in the air will decrease if concentrated $\mathrm{N}_{2} \mathrm{O}$ is present in the mixtures. For example, an $\mathrm{NH}_{3}-\mathrm{N}_{2} \mathrm{O}$-air mixture with a ratio of 13.2-19.8-67.0 is flammable, although the ammonia volume percent is 13.2 and is lower than the $\mathrm{LFL}_{\mathrm{NH} 3}$ of $15 \%$ in air. This fuel-oxidizer mixture will be flammable with the ammonia volume percent as low as $8 \%$ if the $\mathrm{N}_{2} \mathrm{O}$ concentration is 65\%. For $\mathrm{NH}_{3}-\mathrm{N}_{2} \mathrm{O}$-air mixtures, Pfahl and Shepherd (Figure 32 in 1997 report) suggest that the ammonia flammability limit will be $15 \%$ if the $\mathrm{N}_{2} \mathrm{O}$ does not exceed $8 \%$. These test results are for turbulent conditions, with an ignition energy of $8 \mathrm{~J}$. For a $\mathrm{CH}_{4}-\mathrm{N}_{2} \mathrm{O}$-air mixture, the data show that the ratio of 4.5-10.1-85.4 is not flammable. For an $\mathrm{H}_{2}-\mathrm{N}_{2} \mathrm{O}$-air mixture, the data show that a mixture with the ratio of 5.7-10-84.3 has incomplete combustion. It should be noted that the ignition energy of $8 \mathrm{~J}$ used in these experiments is very large 
compared to the energy in most electrostatic discharge sparks (typically a few millijoules) that might occur inside a Hanford Site tank.

This information suggests that the flammability limit established in the fuel-air mixture for each gas will not be reduced unless the $\mathrm{N}_{2} \mathrm{O}$ volume percent exceeds $8 \%$. In this evaluation, the volume percents of 4,15 , and $5 \%$ will be used for $\mathrm{LFL}_{\mathrm{H} 2}, \mathrm{LFL}_{\mathrm{NH} 3}$, and $\mathrm{LFL}_{\mathrm{CH} 4}$, respectively, in Equation 4-3. In each case, the concentration of nitrous oxide was evaluated and did not exceed $8 \%$, therefore, the oxidizer effect of lowering these flammability limits can be ignored.

For ventilation interruptions, the time for the headspace to build up to flammable gas concentrations exceeding the LFL is one of the main concerns of this evaluation.

A time-dependent flammability level, $\mathrm{LFL}_{\mathrm{f}}(\mathrm{t})$, of the mixture can be calculated using the time-dependent gas concentration and Le Chatelier's principle as provided in Equation 4-4:

$$
L F L_{f}(t)=\frac{\left[\mathrm{H}_{2}\right](t)}{L F L_{\mathrm{H}_{2}}}+\frac{\left[\mathrm{NH}_{3}\right](t)}{L F L_{\mathrm{NH}_{3}}}+\frac{\left[\mathrm{CH}_{4}\right](t)}{L F L_{\mathrm{CH}_{4}}}
$$

where:

$$
\begin{aligned}
{\left[\mathrm{H}_{2}\right](\mathrm{t}) } & =\text { hydrogen concentration (volume percent) at time } \mathrm{t} \\
{\left[\mathrm{NH}_{3}\right](\mathrm{t}) } & =\text { ammonia concentration (volume percent) at time } \mathrm{t} \\
{\left[\mathrm{CH}_{4}\right](\mathrm{t}) } & =\text { methane concentration (volume percent) at time } \mathrm{t} \\
\mathrm{LFL}_{\mathrm{H}_{2}} & =\text { hydrogen LFL in air alone, } 4 \% \\
\mathrm{LFL}_{\mathrm{NH}_{3}} & =\text { ammonia LFL in air alone, } 15 \% \\
\mathrm{LFL}_{\mathrm{CH}_{4}} & =\text { methane LFL in air alone, } 5 \%
\end{aligned}
$$

\subsection{TIME TO LFL WITH VENTILATION}

Under normal operating conditions, ventilation can be either active or passive. All DSTs are equipped with active ventilation systems. For active ventilation systems, the main ventilation flow is designed to go through an inlet filter. The 241-AP Tank Farm is an exception to this system; inlet air flows through cover blocks or gaps in riser covers, and other tank openings. The size of the inlet openings was designed to be either fully adjustable or to have just an on-off valve. These filtered inlets are used to control the ventilation rate and to adjust the negative pressure. For passively ventilated tanks, the ventilation flow goes mainly through high-efficiency particulate air (HEPA) breather filters.

Under normal operating conditions, the active ventilation rates range from 100 to $700 \mathrm{ft}^{3} / \mathrm{min}$, while the passive ventilation rates range from 0.5 to $25 \mathrm{ft}^{3} / \mathrm{min}$. On actively ventilated tanks, the off-normal operating ventilation condition considered in the flammability assessment is that the ventilation system is shut down and the air inlet is closed; on passively ventilated tanks, the HEPA breather filter isolation valve is closed. For these conditions, the only vent flow is through the gaps between the cover blocks or riser covers. The off-normal condition allows three possible results: zero ventilation, ventilation from barometric breathing, or ventilation flow caused by natural breathing, which is a combination of barometric breathing, thermal convection, 
venturi effects, etc. The barometric breathing rate, $\mathrm{V}_{\mathrm{bb}}$, is estimated as $0.45 \%$ of the headspace volume per day (WHC-EP-0651) and is given in Equation 4-5:

$$
V_{b b}=0.45 \% \cdot V o l_{\text {headspace }}\left(\frac{f t^{3}}{d a y}\right)
$$

where:

$$
\mathrm{Vol}_{\text {headspace }}=\text { volume of the tank head space, } \mathrm{ft}^{3} \text {. }
$$

During off-normal operating conditions, the most likely ventilation rate is either natural breathing or barometric breathing. The natural breathing rate is estimated to be 5 to 10 times the barometric breathing rate. The zero-ventilation condition is considered to be impossible because of the many openings into the tanks. However, since 1980, an effort has been made to seal off the openings as much as possible for SSTs to prevent water intrusion. The barometric breathing rate was used for the DST and SST ventilation rate under off-normal operating conditions.

Gas concentration can be formulated from a mass balance as provided in Equation 4-6:

$$
\operatorname{Vol} \frac{\partial\left\lfloor C_{g}\right\rfloor}{\partial t}=R_{g}-V_{r} \cdot\left[C_{g}\right]
$$

where:

$$
\begin{aligned}
& {\left[\mathrm{C}_{\mathrm{g}}\right]=\text { gas concentration, volume percent }} \\
& \mathrm{Vol}_{\mathrm{ol}}=\text { volume of the tank headspace, volume } \\
& \mathrm{R}_{\mathrm{g}}=\text { gas release rate from the waste surface, volume/time } \\
& \mathrm{V}_{\mathrm{r}}=\text { ventilation rate in the headspace, volume/time }
\end{aligned}
$$

Equation 4-6 can be changed to reflect diffusion losses rather than ventilation losses. Assuming that there is zero ventilation, but the gas can diffuse out through the concrete dome, then $\mathrm{V}_{\mathrm{r}} \cdot\left[\mathrm{C}_{\mathrm{g}}\right]$ term in Equation 4-6 is changed to the gas diffusion rate (see Section 4.4). 
As given in Equation 4-6, the gas exhaust rate is simply the gas concentration times the output ventilation rate. By solving the differential Equation 4-6, a time-dependent gas concentration at time $t,\left[C_{g}\right](t)$, in units of volume percent is given in Equation 4-7:

$$
\left[C_{g}\right]_{\%}(t)=\left[C_{g}\right]_{\%}\left(t_{0}\right) \exp (-\lambda t)+\left[\frac{R_{g}}{V_{r}}\right]_{\%}[1-\exp (-\lambda t)]
$$

with

$$
\lambda \quad=\mathrm{V}_{\mathrm{r}} / \mathrm{Vol}
$$

where:

$$
\begin{array}{ll}
\mathrm{R}_{\mathrm{g}} & =\text { hydrogen release rate from the waste surface, volume/time } \\
\mathrm{V}_{\mathrm{r}} & =\text { ventilation rate in the headspace, volume/time } \\
{\left[\mathrm{C}_{\mathrm{g}}\right]\left(\mathrm{t}_{\mathrm{o}}\right)} & =\text { initial gas concentration at time } \mathrm{t}, \text { volume percent } \\
\mathrm{Vol} & =\text { volume of the tank headspace, volume } \\
\lambda & =\text { decay parameter from the venting, inverse time } \\
\mathrm{t} & =\text { time. }
\end{array}
$$

In Equation 4-7, the first term represents how the initial gas concentration decays away exponentially with the factor of $\mathrm{e}^{\left(-\lambda^{t}\right)}$ and second term represents how the gas level builds up in the form of $\left[R_{g} / V_{r}\right]_{\%}$ with the factor of $\left[1-e^{\left(-\lambda^{t}\right)}\right]$ as time goes on.

\subsection{TIME TO LFL WITH ZERO VENTILATION}

Under zero ventilation, the only gas release mechanism from the headspace is gaseous diffusion through the tank domes. For the case of gas exiting by diffusion through the dome, the mass balance equation can be written as a differential equation as given in Equation 4-8:

$$
\operatorname{Vol} \frac{\partial\left\lfloor C_{g}\right\rfloor}{\partial t}=R_{g}-A \times Q=R_{g}-A \times \frac{1}{\sum_{i} \frac{L_{i}}{D_{i}}} \times\left(\left[C_{g}\right]-\left[C_{o}\right]\right)
$$

where:

$$
\begin{aligned}
& {\left[\mathrm{C}_{\mathrm{g}}\right]=\text { gas concentration in the tank headspace, volume percent }} \\
& {\left[\mathrm{C}_{\mathrm{o}}\right]=\text { gas concentration outside the tank, volume percent }} \\
& \mathrm{Vol}=\text { volume of the tank headspace, volume } \\
& \mathrm{R}_{\mathrm{g}}=\text { gas release rate from the waste surface, volume/time } \\
& \mathrm{Q}=\text { the diffusion flux, concentration/area-time } \\
& \mathrm{A}=\text { the area of the dome to diffuse, area } \\
& \mathrm{D}_{\mathrm{i}}=\text { the } \mathrm{i}^{\text {th }} \text { layer effective diffusivity, area/time } \\
& \mathrm{L}_{\mathrm{i}}=\text { the thickness of the } \mathrm{i}^{\text {th }} \text { layer to diffuse, length. }
\end{aligned}
$$

The gas exhaust rate is the gas diffusion rate in Equation 4-8, which is the diffusion flux, $\mathrm{Q}_{\mathrm{i}}$, times the surface area of the dome. The diffusion flux is the gas concentration gradient between the headspace and outside over the path-length for diffusion times the diffusivity. The summation of the diffusion rates in Equation 4-8 accounts for several diffusion resistances in series (e.g., concrete, asphaltic membrane, gunite, and soil for a tank dome). By solving the 
differential Equation 4-8, a time-dependent gas concentration at time $t,\left[\mathrm{C}_{\mathrm{g}}\right](\mathrm{t})$, in units of volume percent is given in Equation 4-9:

$$
\left[C_{g}\right]_{\%}(t)=\left[C_{g}\right]_{\%}\left(t_{0}\right) \exp (-\lambda t)+\left[\frac{R_{g}}{\left.A \times \frac{1}{\sum_{i} \frac{L_{i}}{D_{i}}}\right]_{\%}}[1-\exp (-\lambda t)]\right.
$$

with

where:

$$
\lambda \quad=\mathrm{Ax}\left[1 / \Sigma\left(\mathrm{L}_{\mathrm{i}} / \mathrm{D}_{\mathrm{i}}\right)\right] / \mathrm{Vol}
$$

$$
\begin{array}{ll}
\mathrm{R}_{\mathrm{g}} & =\text { hydrogen release rate from the waste surface, volume/time } \\
{\left[\mathrm{C}_{\mathrm{g}}\right]\left(\mathrm{t}_{\mathbf{o}}\right)} & =\text { initial gas concentration at time } \mathrm{t}, \text { volume percent } \\
\mathrm{Vol} & =\text { volume of the tank headspace, volume } \\
\lambda & =\text { decay parameter from the diffusion, inverse time } \\
\mathrm{t} & =\text { time. }
\end{array}
$$

\subsection{MINIMUM VENTILATION RATE TO MAINTAIN HEADSPACE BELOW 25 OR 100 PERCENT OF THE LFL}

Whether the headspace of a tank will reach the specified flammability limit or not depends on the balance between the gas release rates and the ventilation rates of the system. A ventilation rate, defined as the minimum vent rate, which the steady-state concentrations will just reach the specified flammability limit can be determined using Equations 4-4 and 4-7. If the headspace ventilation rate is larger than this minimum, the system will never reach the specified flammability limit. Ventilation rate is embedded in Equation 4-7, and a macro function is used to calculate the minimum ventilation needed to stay below the specified flammability limit (see Section 6.0). 


\subsection{INPUT DATA}

The calculation requires the following concentration inputs to calculate HGR in the liquid: sodium $(\mathrm{Na})$, aluminum $(\mathrm{Al})$, hydroxide $(\mathrm{OH})$, nitrite $\left(\mathrm{NO}_{2}\right)$, nitrate $\left(\mathrm{NO}_{3}\right)$, and total organic carbon (TOC). The following radionuclide concentrations are also required: cesium-137 $\left({ }^{137} \mathrm{Cs}\right)$, strontium-90 $\left({ }^{90} \mathrm{Sr}\right)$, americium-241 $\left({ }^{241} \mathrm{Am}\right)$, plutonium-238 $\left({ }^{238} \mathrm{Pu}\right)$, plutonium-239 $\left({ }^{239} \mathrm{Pu}\right)$, and plutonium-240 $\left({ }^{240} \mathrm{Pu}\right)$. The peak annual averaged waste and headspace temperatures (or conservatively just the annual peak waste and headspace temperatures), liquid and bulk water concentrations (wt\%), and total waste volumes are also needed. Bulk and liquid specific gravity is required to convert waste volumes into total waste mass. All the above data is needed for both supernatant and solid layers. The Best Basis Inventory (BBI), Tank Characterization Database (TCD) accessed through the Tank Waste Information Network System (TWINS), and the Surveillance Analysis Computer System (SACS) were queried to obtain the required waste properties and compositions and are shown in Appendix A. Data missing from the database were estimated based on the methodology presented in SNL-000198.

The steps described below were necessary to convert raw BBI data to the format required for input data. Inputs and results are documented in a spreadsheet (Raw Input Data for RPP5926_v2.xls, SVF-1570 Rev. 1).

In BBI detail calculations, more than one density was used in a waste layer for a number of tanks. A single representative density for each waste layer was calculated by weighted averaging and results are shown in Appendix A.

Data are required from the supernatant liquid layer, solid layer (the waste below the supernatant liquid layer), and the total waste. Waste compositions from various solid layer waste types were weight averaged.

Concentrations of TOC, nitrate ion, nitrite ion, and aluminum in the liquid phase and the liquid density for solid layer were assigned the same value as the supernatant liquid layer if no interstitial liquid data were available.

$\mathrm{Al}, \mathrm{Na}, \mathrm{NO}_{2}, \mathrm{NO}_{3}, \mathrm{OH}$ and TOC input concentrations were calculated by dividing the inventory of each analyte by waste volume as follows

$$
\text { concentration }\left(\frac{\mu \mathrm{g}}{\mathrm{mL}}\right)=\frac{10^{9}\left(\frac{\mu \mathrm{g}}{\mathrm{kg}}\right) \times \text { Inventory }(\mathrm{kg})}{\text { Volume }(\mathrm{kL}) \times 10^{6}\left(\frac{\mathrm{mL}}{\mathrm{kL}}\right)}
$$

Radionuclide input concentrations were calculated by dividing total inventory by waste volume and representative density as follows:

$$
\text { concentration }\left(\frac{\mu \mathrm{Ci}}{\mathrm{g}}\right)=\frac{10^{6}\left(\frac{\mu \mathrm{Ci}}{\mathrm{Ci}}\right) \times \text { Inventory }(\mathrm{Ci})}{\text { Volume }(\mathrm{kL}) \times 10^{6}\left(\frac{\mathrm{mL}}{\mathrm{kL}}\right) \times \rho\left(\frac{\mathrm{g}}{\mathrm{mL}}\right)}
$$

In BBI development, the total waste volume is divided into three parts: solid, liquid, and retained gas (if there is any). In the detail calculation report, the volume of retained gas is not 
reported because it has no contribution to the analytes inventory. Although the retained gas has no contribution to the HGR calculations, it is part of the total waste volume and will impact the headspace volume calculation, and thus the flammability calculations. The reported retained gas volume is included in determining the waste depth and total waste volume for flammability calculations.

Tank waste and headspace temperature data were obtained for the October 1, 2008 to October 1, 2009 time period. The data were reviewed to find the maximum waste and headspace temperatures for the year and these bounding data (excluding suspect data) were used to calculate TTLFL in this revision except for SSTs 241-A-103 and 241-BY-110. Maximum temperatures were averaged for each waste layer for these two tanks to produce reasonably conservative temperatures. That is, multiple temperature readings within the waste layers were averaged for the day in which the thermocouples measured highest for the year. Using yearly bounding maximums or averaged waste layer maximums conservatively includes seasonal effects and waste tank operations that occurred throughout the year.

Periodic monitoring of DST waste temperatures is conducted to ensure that transfers or storage conditions have not resulted in unanticipated increases in DST waste temperatures. The SST temperature trends are discussed in Appendix D. All SSTs are now passively ventilated (except that active ventilation may be used to support some retrieval activities). All SSTs have flat or decreasing temperature trends. Temperature increases are not possible without significant changes to the waste. Therefore, the $5^{\circ} \mathrm{C}$ added to DST temperatures is not added to SST temperatures and routine temperature monitoring in the SSTs is not required. Significant changes in SST status (e.g., retrieval activities or large chemical additions) are evaluated through the waste compatibility program.

Tank capacity, headspace volume, and diameter are inputs for calculating fill fractions and the wetted surface area for corrosion. These values were obtained from RPP-13019. Gaseous diffusion through SST domes requires knowing the surface area, thickness, and diffusivity through the concrete domes. Thickness and diffusivities for asphaltic membrane, gunite, and soil are also required and were taken from RPP-18491. Data used for gaseous diffusion are shown in Appendix B.

Calculations assume an initial flammability of $2 \%$ of the LFL to account for ammonia contribution. The basis for ammonia contribution to flammability is given in Appendix C.

Radionuclide concentrations are given in curies and need to be converted to watts. Curie to watt conversions are reported in HNF-EP-0063 and shown in Table 5-1.

Table 5-1. Curie to Watt Conversion Factors.

\begin{tabular}{|c|c|c|c|c|c|}
\hline $\begin{array}{c}{ }^{137} \mathbf{C s}^{*} \\
\text { (watt/Ci) }\end{array}$ & $\begin{array}{c}{ }^{90} \mathrm{Sr}^{*} \\
\text { (watt/Ci) }\end{array}$ & $\begin{array}{c}{ }^{241} \mathbf{A m} \\
\text { (watt/Ci) }\end{array}$ & $\begin{array}{c}{ }^{238} \mathbf{P u} \\
(\text { watt/Ci) }\end{array}$ & $\begin{array}{c}{ }^{239} \mathbf{P u} \\
\text { (watt/Ci) }\end{array}$ & $\begin{array}{c}{ }^{240} \mathbf{P u} \\
\text { (watt/Ci) }\end{array}$ \\
\hline $4.82 \mathrm{E}-03$ & $6.70 \mathrm{E}-03$ & $3.34 \mathrm{E}-02$ & $3.32 \mathrm{E}-02$ & $3.11 \mathrm{E}-02$ & $3.12 \mathrm{E}-02$ \\
\hline
\end{tabular}

Note: ${ }^{*}$ Includes daughters with half-life less than parent radionuclides. 


\subsection{COMPUTER SOFTWARE}

TTLFL is calculated by a multiuse spreadsheet that uses Microsoft Excel ${ }^{1} 2007$ software. The file name is DST_and_SST_TTLFL_v4.xlsm. The Spreadsheet Verification and Release Form number is SVF-1536 Rev 3. One macro is used in the spreadsheet to calculate TTLFL. The TTLFL equations required an iteration-based macro function to be created. The TTLFL macro increases time by 0.1 days until the specified flammability (either 25 or $100 \%$ of the LFL) calculated by Equation 4-4 is reached.

\footnotetext{
${ }^{1}$ Excel is a registered trademark of Microsoft Corporation, Redmond, Washington.
} 


\subsection{RESULTS}

Time to reach 25 and $100 \%$ LFLs was calculated for tank headspaces under barometric breathing and zero ventilation conditions. Sections 7.1 and 7.2 present results for DSTs and SSTs, respectively.

\subsection{DOUBLE-SHELL TANK RESULTS}

Table 7-1 presents results for DSTs under barometric breathing, while Table 7-2 gives results for zero ventilation. Table 7-3 shows hydrogen generation in each DST and minimum ventilation rates to keep flammability below 25 and $100 \%$ of the LFL. Results in all three tables include a $5^{\circ} \mathrm{C}$ temperature increase and 10,000 gallon water addition.

Table 7-1. Double-Shell Tank Steady-State Flammable Gas Concentration and

Time to Lower Flammability Limit Under Barometric Breathing.

\begin{tabular}{|c|c|c|c|}
\hline Tank & $\begin{array}{c}\text { Steady-State Concentration } \\
\text { (\% of LFL) }\end{array}$ & $\begin{array}{l}\text { Time to Reach 25\% of LFL } \\
\text { (days) }\end{array}$ & $\begin{array}{l}\text { Time to Reach } 100 \% \text { of LFL } \\
\text { (days) }\end{array}$ \\
\hline 241-AN-101 & 66 & 98 & NA \\
\hline 241-AN-102 & $256 \%$ & 21 & 108 \\
\hline 241-AN-103 & $77 \%$ & 81 & NA \\
\hline 241-AN-104 & $113 \%$ & 52 & 477 \\
\hline $241-\mathrm{AN}-105$ & $101 \%$ & 59 & 1109 \\
\hline $241-\mathrm{AN}-106$ & $47 \%$ & 158 & NA \\
\hline $241-\mathrm{AN}-107$ & $238 \%$ & 23 & 119 \\
\hline 241-AP-101 & $21 \%$ & NA & NA \\
\hline 241-AP-102 & $31 \%$ & 338 & NA \\
\hline 241-AP-103 & $54 \%$ & 130 & NA \\
\hline 241-AP-104 & $70 \%$ & 91 & NA \\
\hline 241-AP-105 & $31 \%$ & 347 & NA \\
\hline 241-AP-106 & $68 \%$ & 94 & NA \\
\hline 241-AP-107 & $57 \%$ & 121 & NA \\
\hline 241-AP-108 & $83 \%$ & 75 & NA \\
\hline 241-AW-101 & $124 \%$ & 47 & 364 \\
\hline 241-AW-102 & $15 \%$ & NA & NA \\
\hline 241-AW-103 & $25 \%$ & 924 & NA \\
\hline $241-A W-104$ & $43 \%$ & 184 & NA \\
\hline 241-AW-105 & $6 \%$ & NA & NA \\
\hline 241-AW-106 & $66 \%$ & 98 & NA \\
\hline 241-AY-101 & $49 \%$ & 151 & NA \\
\hline 241-AY-102 & $365 \%$ & 15 & 70 \\
\hline 241-AZ-101 & $490 \%$ & 11 & 50 \\
\hline 241-AZ-102 & $96 \%$ & 62 & NA \\
\hline 241-SY-101 & $20 \%$ & NA & NA \\
\hline 241-SY-102 & $9 \%$ & NA & NA \\
\hline 241-SY-103 & $44 \%$ & 175 & NA \\
\hline
\end{tabular}

NA = Not Applicable, headspace will not reach specified flammability level. 


\section{RPP-5926 REV 9}

Table 7-2. Double-Shell Tank Time to Lower Flammability Limit Under Zero Ventilation.

\begin{tabular}{|c|c|c|}
\hline Tank & $\begin{array}{l}\text { Time to Reach } 25 \% \text { of LFL } \\
\text { (days) }\end{array}$ & $\begin{array}{l}\text { Time to Reach } 100 \% \text { of LFL } \\
\text { (days) }\end{array}$ \\
\hline 241-AN-101 & 79 & 338 \\
\hline 241-AN-102 & 20 & 86 \\
\hline 241-AN-103 & 68 & 289 \\
\hline 241-AN-104 & 46 & 196 \\
\hline 241-AN-105 & 52 & 221 \\
\hline 241-AN-106 & 113 & 483 \\
\hline 241-AN-107 & 22 & 92 \\
\hline 241-AP-101 & 273 & 1163 \\
\hline 241-AP-102 & 173 & 740 \\
\hline 241-AP-103 & 98 & 420 \\
\hline $241-\mathrm{AP}-104$ & 75 & 320 \\
\hline 241-AP-105 & 175 & 748 \\
\hline 241-AP-106 & 77 & 328 \\
\hline 241-AP-107 & 93 & 398 \\
\hline 241-AP-108 & 63 & 270 \\
\hline 241-AW-101 & 42 & 179 \\
\hline 241-AW-102 & 392 & 1669 \\
\hline 241-AW-103 & 219 & 932 \\
\hline 241-AW-104 & 125 & 534 \\
\hline 241-AW-105 & 1373 & $>1826$ \\
\hline 241-AW-106 & 79 & 338 \\
\hline $241-\mathrm{AY}-101$ & 110 & 467 \\
\hline 241-AY-102 & 14 & 60 \\
\hline $241-A Z-101$ & 10 & 45 \\
\hline $241-A Z-102$ & 54 & 232 \\
\hline 241-SY-101 & 277 & 1180 \\
\hline 241-SY-102 & 684 & $>1826$ \\
\hline 241-SY-103 & 121 & 516 \\
\hline
\end{tabular}


Table 7-3. Double-Shell Tank Hydrogen Generation Rate and Minimum Ventilation Rate Required to Maintain Headspace Flammable Gas Concentration Below Specified Level.

\begin{tabular}{|c|c|c|c|}
\hline Tank & $\begin{array}{c}\text { Total HGR } \\
\left(\mathbf{f t}^{\mathbf{3}} \text { /day) }\right.\end{array}$ & $\begin{array}{c}\text { Ventilation rate required to } \\
\text { stay below 25\% of LFL } \\
\left(\mathbf{f t}^{\mathbf{3}} \text { /min) }\right.\end{array}$ & $\begin{array}{c}\text { Ventilation rate required to } \\
\text { stay below 100\% of LFL } \\
\left(\mathbf{f t}^{\mathbf{3}} \mathbf{\text { min }}\right)\end{array}$ \\
\hline $241-\mathrm{AN}-101$ & 3.7 & 0.30 & $\mathbf{0 . 0 7 0}$ \\
\hline $241-\mathrm{AN}-102$ & 19 & 1.5 & 0.36 \\
\hline $241-\mathrm{AN}-103$ & 6.0 & 0.49 & 0.12 \\
\hline $241-\mathrm{AN}-104$ & 7.5 & 0.61 & 0.14 \\
\hline $241-\mathrm{AN}-105$ & 5.2 & 0.42 & 0.099 \\
\hline $241-\mathrm{AN}-106$ & 6.4 & 0.52 & 0.12 \\
\hline $241-\mathrm{AN}-107$ & 16 & 1.3 & 0.30 \\
\hline $241-\mathrm{AP}-101$ & 1.0 & 0.083 & 0.019 \\
\hline $241-\mathrm{AP}-102$ & 2.1 & 0.17 & 0.039 \\
\hline $241-\mathrm{AP}-103$ & 3.0 & 0.25 & 0.058 \\
\hline $241-\mathrm{AP}-104$ & 14 & 1.1 & 0.26 \\
\hline $241-\mathrm{AP}-105$ & 2.7 & 0.22 & 0.052 \\
\hline $241-\mathrm{AP}-106$ & 3.8 & 0.31 & 0.073 \\
\hline $241-\mathrm{AP}-107$ & 3.4 & 0.27 & 0.064 \\
\hline $241-\mathrm{AP}-108$ & 2.7 & 0.22 & 0.052 \\
\hline $241-\mathrm{AW}-101$ & 6.5 & 0.53 & 0.12 \\
\hline $241-\mathrm{AW}-102$ & 1.0 & 0.080 & 0.019 \\
\hline $241-\mathrm{AW}-103$ & 1.6 & 0.13 & 0.030 \\
\hline $241-\mathrm{AW}-104$ & 3.0 & 0.25 & 0.058 \\
\hline $241-\mathrm{AW}-105$ & 0.8 & 0.066 & 0.016 \\
\hline $241-\mathrm{AW}-106$ & 3.7 & 0.30 & 0.071 \\
\hline $241-\mathrm{AY}-101$ & 4.6 & 0.37 & 0.086 \\
\hline $241-\mathrm{AY}-102$ & 36 & 2.9 & 0.68 \\
\hline $241-\mathrm{AZ}-101$ & 43 & 3.5 & 0.82 \\
\hline $241-\mathrm{AZ}-102$ & 25 & 2.1 & 0.49 \\
\hline $241-\mathrm{SY}-101$ & 1.1 & 0.087 & 0.020 \\
\hline $241-\mathrm{SY}-102$ & 1.4 & 0.11 & 0.026 \\
\hline $241-\mathrm{SY}-103$ & 5.8 & 0.47 & 0.11 \\
\hline
\end{tabular}




\subsection{SINGLE-SHELL TANK RESULTS}

Table 7-4 presents results for SSTs under barometric breathing, while Table 7-5 gives results for zero ventilation. Mass transfer through barometric breathing greatly exceeds that for dome diffusion. Table 7-4 results neglect diffusion losses, while Table 7-5 includes hydrogen and methane diffusion through the SST domes. Table 7-6 shows hydrogen generation in each SST and minimum ventilation rates to keep flammability below 25 and $100 \%$ of the LFL. Minimum ventilation rates also neglect diffusion mass transfer. Results in all three tables include a hypothetical 10,000 gallon water addition in the larger 100-series tanks and a 1,000 gallon water addition in the smaller 200-Series tanks.

Table 7-4. Single-Shell Tank Steady-State Flammable Gas Concentration and

Time to Lower Flammability Limit Under Barometric Breathing.

\begin{tabular}{|c|c|c|c|}
\hline Tank & $\begin{array}{c}\text { Steady-State Concentration } \\
(\% \text { of LFL) }\end{array}$ & $\begin{array}{c}\text { Time to Reach 25\% of LFL } \\
\text { (days) }\end{array}$ & $\begin{array}{l}\text { Time to Reach } 100 \% \text { of LFL } \\
\text { (days) }\end{array}$ \\
\hline $241-A-101$ & \begin{tabular}{|c|}
$10 \%$ \\
\end{tabular} & NA & NA \\
\hline $241-A-102$ & $4 \%$ & NA & NA \\
\hline $241-A-103$ & $14 \%$ & NA & NA \\
\hline 241-A-104 & $3 \%$ & NA & NA \\
\hline $241-\mathrm{A}-105$ & $3 \%$ & NA & NA \\
\hline $241-A-106$ & $14 \%$ & NA & NA \\
\hline $241-\mathrm{AX}-101$ & $11 \%$ & NA & NA \\
\hline 241-AX-102 & $4 \%$ & NA & NA \\
\hline 241-AX-103 & $6 \%$ & NA & NA \\
\hline 241-AX-104 & $5 \%$ & NA & NA \\
\hline 241-B-101 & $5 \%$ & NA & $\mathrm{NA}$ \\
\hline 241-B-102 & $4 \%$ & NA & NA \\
\hline $241-\mathrm{B}-103$ & $4 \%$ & NA & NA \\
\hline $241-\mathrm{B}-104$ & $7 \%$ & NA & NA \\
\hline $241-\mathrm{B}-105$ & $5 \%$ & NA & NA \\
\hline 241-B-106 & $4 \%$ & NA & NA \\
\hline 241-B-107 & $4 \%$ & NA & NA \\
\hline 241-B-108 & $4 \%$ & NA & NA \\
\hline 241-B-109 & $4 \%$ & NA & NA \\
\hline 241-B-110 & $7 \%$ & NA & NA \\
\hline 241-B-111 & $11 \%$ & NA & NA \\
\hline $241-\mathrm{B}-112$ & $4 \%$ & NA & NA \\
\hline $241-\mathrm{B}-201$ & $14 \%$ & NA & NA \\
\hline 241-B-202 & $13 \%$ & NA & NA \\
\hline 241-B-203 & $155 \%$ & 36 & 228 \\
\hline 241-B-204 & $140 \%$ & 41 & 276 \\
\hline 241-BX-101 & $4 \%$ & NA & NA \\
\hline 241-BX-102 & $5 \%$ & NA & NA \\
\hline 241-BX-103 & $8 \%$ & NA & NA \\
\hline 241-BX-104 & $5 \%$ & NA & NA \\
\hline 241-BX-105 & $4 \%$ & NA & NA \\
\hline 241-BX-106 & $4 \%$ & NA & NA \\
\hline 241-BX-107 & $7 \%$ & NA & NA \\
\hline 241-BX-108 & $4 \%$ & NA & NA \\
\hline $241-\mathrm{BX}-109$ & $7 \%$ & NA & NA \\
\hline $241-\mathrm{BX}-110$ & $6 \%$ & NA & NA \\
\hline 241-BX-111 & $5 \%$ & NA & NA \\
\hline
\end{tabular}


Table 7-4. Single-Shell Tank Steady-State Flammable Gas Concentration and

Time to Lower Flammability Limit Under Barometric Breathing.

\begin{tabular}{|c|c|c|c|}
\hline Tank & \begin{tabular}{|c|}
$\begin{array}{c}\text { Steady-State Concentration } \\
(\% \text { of LFL })\end{array}$ \\
\end{tabular} & $\begin{array}{c}\text { Time to Reach } 25 \% \text { of LFL } \\
\text { (days) }\end{array}$ & \begin{tabular}{|c}
$\begin{array}{c}\text { Time to Reach } 100 \% \text { of LFL } \\
\text { (days) }\end{array}$ \\
\end{tabular} \\
\hline 241-BX-112 & $5 \%$ & $\mathrm{NA}$ & NA \\
\hline 241-BY-101 & $9 \%$ & NA & NA \\
\hline 241-BY-102 & $5 \%$ & NA & NA \\
\hline 241-BY-103 & $6 \%$ & NA & NA \\
\hline 241-BY-104 & $13 \%$ & NA & NA \\
\hline 241-BY-105 & $9 \%$ & NA & NA \\
\hline 241-BY-106 & $11 \%$ & NA & NA \\
\hline 241-BY-107 & $6 \%$ & NA & NA \\
\hline 241-BY-108 & $6 \%$ & NA & NA \\
\hline 241-BY-109 & $5 \%$ & NA & NA \\
\hline 241-BY-110 & $19 \%$ & NA & NA \\
\hline 241-BY-111 & $6 \%$ & NA & NA \\
\hline 241-BY-112 & $5 \%$ & NA & NA \\
\hline $241-C-101$ & $4 \%$ & NA & NA \\
\hline $241-C-102$ & $8 \%$ & NA & NA \\
\hline $241-C-103$ & $6 \%$ & NA & NA \\
\hline $241-C-104$ & $17 \%$ & NA & NA \\
\hline $241-C-105$ & $8 \%$ & NA & NA \\
\hline $241-C-106$ & $9 \%$ & NA & NA \\
\hline $241-C-107$ & $42 \%$ & 188 & NA \\
\hline $241-C-108$ & $6 \%$ & NA & NA \\
\hline 241-C-109 & $4 \%$ & NA & NA \\
\hline $241-C-110$ & $4 \%$ & NA & NA \\
\hline $241-C-111$ & $11 \%$ & NA & NA \\
\hline $241-C-112$ & $13 \%$ & NA & NA \\
\hline 241-C-201 & $7 \%$ & NA & NA \\
\hline $241-C-202$ & $7 \%$ & NA & NA \\
\hline $241-C-203$ & $6 \%$ & NA & NA \\
\hline 241-C-204 & $6 \%$ & NA & NA \\
\hline $241-S-101$ & $10 \%$ & NA & NA \\
\hline 241-S-102 & $4 \%$ & NA & NA \\
\hline $241-S-103$ & $6 \%$ & NA & NA \\
\hline 241-S-104 & $7 \%$ & NA & NA \\
\hline 241-S-105 & $6 \%$ & NA & NA \\
\hline 241-S-106 & $7 \%$ & NA & NA \\
\hline 241-S-107 & $8 \%$ & NA & NA \\
\hline $241-S-108$ & $7 \%$ & NA & NA \\
\hline 241-S-109 & $7 \%$ & NA & NA \\
\hline $241-S-110$ & $8 \%$ & NA & NA \\
\hline 241-S-111 & $9 \%$ & NA & NA \\
\hline $241-S-112$ & $5 \%$ & NA & NA \\
\hline 241-SX-101 & $7 \%$ & NA & NA \\
\hline 241-SX-102 & $13 \%$ & NA & NA \\
\hline 241-SX-103 & $52 \%$ & 135 & NA \\
\hline 241-SX-104 & $21 \%$ & NA & NA \\
\hline 241-SX-105 & $40 \%$ & 202 & NA \\
\hline 241-SX-106 & $7 \%$ & NA & NA \\
\hline 241-SX-107 & $10 \%$ & NA & NA \\
\hline 241-SX-108 & $5 \%$ & NA & NA \\
\hline 241-SX-109 & $32 \%$ & 329 & NA \\
\hline
\end{tabular}


Table 7-4. Single-Shell Tank Steady-State Flammable Gas Concentration and

Time to Lower Flammability Limit Under Barometric Breathing.

\begin{tabular}{|c|c|c|c|}
\hline Tank & $\begin{array}{c}\text { Steady-State Concentration } \\
\text { (\% of LFL) }\end{array}$ & $\begin{array}{l}\text { Time to Reach 25\% of LFL } \\
\text { (days) }\end{array}$ & $\begin{array}{l}\text { Time to Reach } 100 \% \text { of LFL } \\
\text { (days) }\end{array}$ \\
\hline $241-S X-110$ & \begin{tabular}{|c|}
$15 \%$ \\
\end{tabular} & NA & NA \\
\hline 241-SX-111 & $19 \%$ & NA & NA \\
\hline $241-\mathrm{SX}-112$ & $7 \%$ & NA & NA \\
\hline $241-S X-113$ & $3 \%$ & NA & NA \\
\hline 241-SX-114 & $20 \%$ & NA & NA \\
\hline 241-SX-115 & $4 \%$ & NA & NA \\
\hline 241-T-101 & $4 \%$ & NA & NA \\
\hline 241-T-102 & $6 \%$ & NA & NA \\
\hline $241-T-103$ & $4 \%$ & NA & NA \\
\hline 241-T-104 & $6 \%$ & NA & NA \\
\hline $241-T-105$ & $5 \%$ & NA & NA \\
\hline $241-T-106$ & $4 \%$ & NA & NA \\
\hline 241-T-107 & $6 \%$ & NA & NA \\
\hline $241-\mathrm{T}-108$ & $4 \%$ & NA & NA \\
\hline 241-T-109 & $4 \%$ & NA & NA \\
\hline 241-T-110 & $13 \%$ & NA & NA \\
\hline $241-T-111$ & $8 \%$ & NA & NA \\
\hline 241-T-112 & $4 \%$ & NA & NA \\
\hline $241-T-201$ & $33 \%$ & 304 & NA \\
\hline 241-T-202 & $9 \%$ & NA & NA \\
\hline $241-T-203$ & $20 \%$ & NA & NA \\
\hline $241-T-204$ & $20 \%$ & NA & NA \\
\hline 241-TX-101 & $4 \%$ & NA & NA \\
\hline 241-TX-102 & $5 \%$ & NA & NA \\
\hline 241-TX-103 & $4 \%$ & NA & NA \\
\hline 241-TX-104 & $4 \%$ & NA & NA \\
\hline 241-TX-105 & $14 \%$ & NA & NA \\
\hline 241-TX-106 & $6 \%$ & NA & NA \\
\hline 241-TX-107 & $3 \%$ & NA & NA \\
\hline 241-TX-108 & $4 \%$ & NA & NA \\
\hline 241-TX-109 & $8 \%$ & NA & NA \\
\hline 241-TX-110 & $8 \%$ & NA & NA \\
\hline 241-TX-111 & $7 \%$ & NA & NA \\
\hline 241-TX-112 & $12 \%$ & NA & NA \\
\hline $241-\mathrm{TX}-113$ & $10 \%$ & NA & NA \\
\hline 241-TX-114 & $8 \%$ & NA & NA \\
\hline 241-TX-115 & $9 \%$ & NA & NA \\
\hline 241-TX-116 & $8 \%$ & NA & NA \\
\hline 241-TX-117 & $6 \%$ & NA & NA \\
\hline 241-TX-118 & $8 \%$ & NA & NA \\
\hline 241-TY-101 & $4 \%$ & NA & NA \\
\hline 241-TY-102 & $4 \%$ & NA & NA \\
\hline 241-TY-103 & $5 \%$ & NA & NA \\
\hline 241-TY-104 & $4 \%$ & NA & NA \\
\hline 241-TY-105 & $6 \%$ & NA & NA \\
\hline 241-TY-106 & $3 \%$ & NA & NA \\
\hline $241-U-101$ & $4 \%$ & NA & NA \\
\hline 241-U-102 & $13 \%$ & NA & NA \\
\hline $241-U-103$ & $21 \%$ & NA & NA \\
\hline 241-U-104 & $4 \%$ & NA & NA \\
\hline
\end{tabular}


Table 7-4. Single-Shell Tank Steady-State Flammable Gas Concentration and Time to Lower Flammability Limit Under Barometric Breathing.

\begin{tabular}{|c|c|c|c|}
\hline Tank & $\begin{array}{c}\text { Steady-State Concentration } \\
\text { (\% of LFL) }\end{array}$ & $\begin{array}{c}\text { Time to Reach 25\% of LFL } \\
\text { (days) }\end{array}$ & $\begin{array}{c}\text { Time to Reach 100\% of LFL } \\
\text { (days) }\end{array}$ \\
\hline $241-U-105$ & $22 \%$ & NA & NA \\
\hline $241-U-106$ & $10 \%$ & NA & NA \\
\hline $241-U-107$ & $7 \%$ & NA & NA \\
\hline $241-U-108$ & $14 \%$ & NA & NA \\
\hline $241-U-109$ & $12 \%$ & NA & NA \\
\hline $241-U-110$ & $6 \%$ & NA & NA \\
\hline $241-U-111$ & $7 \%$ & NA & NA \\
\hline $241-U-112$ & $4 \%$ & NA & NA \\
\hline $241-U-201$ & $4 \%$ & NA & NA \\
\hline $241-U-202$ & $4 \%$ & NA & NA \\
\hline $241-U-203$ & $4 \%$ & NA & NA \\
\hline $241-U-204$ & $7 \%$ & NA & \\
\hline
\end{tabular}

NA = Not Applicable, headspace will not reach specified flammability level.

Table 7-5. Single-Shell Tank Time to Lower Flammability Limit Under Zero Ventilation.

\begin{tabular}{|c|c|c|}
\hline Tank & $\begin{array}{l}\text { Time to Reach } 25 \% \text { of LFL } \\
\text { (days) }\end{array}$ & $\begin{array}{l}\text { Time to Reach } 100 \% \text { of LFL } \\
\text { (days) }\end{array}$ \\
\hline $241-A-101$ & 635 & $>1826$ \\
\hline $241-\mathrm{A}-102$ & $>1826$ & $>1826$ \\
\hline $241-\mathrm{A}-103$ & 427 & $>1826$ \\
\hline $241-\mathrm{A}-104$ & $>1826$ & $>1826$ \\
\hline $241-\mathrm{A}-105$ & $>1826$ & $>1826$ \\
\hline $241-A-106$ & 434 & $>1826$ \\
\hline 241-AX-101 & not occur & not occur \\
\hline $241-\mathrm{AX}-102$ & not occur & not occur \\
\hline $241-A X-103$ & not occur & not occur \\
\hline $241-A X-104$ & not occur & not occur \\
\hline $241-B-101$ & 1728 & $>1826$ \\
\hline 241-B-102 & $>1826$ & $>1826$ \\
\hline 241-B-103 & $>1826$ & $>1826$ \\
\hline 241-B-104 & 1196 & $>1826$ \\
\hline 241-B-105 & 1588 & $>1826$ \\
\hline $241-B-106$ & $>1826$ & $>1826$ \\
\hline 241-B-107 & $>1826$ & $>1826$ \\
\hline 241-B-108 & $>1826$ & $>1826$ \\
\hline 241-B-109 & $>1826$ & $>1826$ \\
\hline $241-B-110$ & 1028 & $>1826$ \\
\hline 241-B-111 & 564 & $>1826$ \\
\hline 241-B-112 & $>1826$ & $>1826$ \\
\hline 241-B-201 & 418 & $>1826$ \\
\hline 241-B-202 & 457 & $>1826$ \\
\hline 241-B-203 & 34 & 145 \\
\hline 241-B-204 & 37 & 160 \\
\hline 241-BX-101 & $>1826$ & $>1826$ \\
\hline 241-BX-102 & $>1826$ & $>1826$ \\
\hline 241-BX-103 & 856 & $>1826$ \\
\hline 241-BX-104 & $>1826$ & $>1826$ \\
\hline 241-BX-105 & $>1826$ & $>1826$ \\
\hline
\end{tabular}


Table 7-5. Single-Shell Tank Time to Lower Flammability Limit Under Zero Ventilation.

\begin{tabular}{|c|c|c|}
\hline Tank & $\begin{array}{c}\text { Time to Reach } 25 \% \text { of LFL } \\
\text { (days) }\end{array}$ & $\begin{array}{l}\text { Time to Reach } 100 \% \text { of LFL } \\
\text { (days) }\end{array}$ \\
\hline 241-BX-106 & $>1826$ & $>1826$ \\
\hline 241-BX-107 & 1101 & $>1826$ \\
\hline 241-BX-108 & $>1826$ & $>1826$ \\
\hline 241-BX-109 & 1176 & $>1826$ \\
\hline 241-BX-110 & 1472 & $>1826$ \\
\hline 241-BX-111 & $>1826$ & $>1826$ \\
\hline $241-\mathrm{BX}-112$ & $>1826$ & $>1826$ \\
\hline 241-BY-101 & 740 & $>1826$ \\
\hline $241-B Y-102$ & $>1826$ & $>1826$ \\
\hline 241-BY-103 & 1230 & $>1826$ \\
\hline 241-BY-104 & 495 & $>1826$ \\
\hline 241-BY-105 & 785 & $>1826$ \\
\hline 241-BY-106 & 588 & $>1826$ \\
\hline 241-BY-107 & 1194 & $>1826$ \\
\hline 241-BY-108 & 1301 & $>1826$ \\
\hline 241-BY-109 & 1783 & $>1826$ \\
\hline 241-BY-110 & 306 & 1358 \\
\hline 241-BY-111 & 1246 & $>1826$ \\
\hline 241-BY-112 & 1623 & $>1826$ \\
\hline $241-\mathrm{C}-101$ & $>1826$ & $>1826$ \\
\hline $241-C-102$ & 825 & $>1826$ \\
\hline $241-C-103$ & 1319 & $>1826$ \\
\hline $241-C-104$ & 335 & 1505 \\
\hline $241-C-105$ & 864 & $>1826$ \\
\hline $241-C-106$ & 761 & $>1826$ \\
\hline $241-C-107$ & 128 & 554 \\
\hline $241-C-108$ & 1318 & $>1826$ \\
\hline $241-C-109$ & $>1826$ & $>1826$ \\
\hline $241-C-110$ & $>1826$ & $>1826$ \\
\hline $241-C-111$ & 561 & $>1826$ \\
\hline $241-C-112$ & 474 & $>1826$ \\
\hline $241-C-201$ & 1155 & $>1826$ \\
\hline $241-C-202$ & 1136 & $>1826$ \\
\hline $241-C-203$ & 1182 & $>1826$ \\
\hline $241-C-204$ & 1195 & $>1826$ \\
\hline $241-S-101$ & 653 & $>1826$ \\
\hline $241-S-102$ & $>1826$ & $>1826$ \\
\hline $241-S-103$ & 1307 & $>1826$ \\
\hline 241-S-104 & 1148 & $>1826$ \\
\hline $241-S-105$ & 1488 & $>1826$ \\
\hline $241-S-106$ & 992 & $>1826$ \\
\hline $241-S-107$ & 857 & $>1826$ \\
\hline 241-S-108 & 1052 & $>1826$ \\
\hline 241-S-109 & 1080 & $>1826$ \\
\hline $241-S-110$ & 926 & $>1826$ \\
\hline 241-S-111 & 795 & $>1826$ \\
\hline $241-S-112$ & 1710 & $>1826$ \\
\hline $241-\mathrm{SX}-101$ & not occur & not occur \\
\hline $241-\mathrm{SX}-102$ & not occur & not occur \\
\hline 241-SX-103 & 280 & not occur \\
\hline 241-SX-104 & not occur & not occur \\
\hline
\end{tabular}


Table 7-5. Single-Shell Tank Time to Lower Flammability Limit Under Zero Ventilation.

\begin{tabular}{|c|c|c|}
\hline Tank & $\begin{array}{l}\text { Time to Reach } 25 \% \text { of LFL } \\
\text { (days) }\end{array}$ & $\begin{array}{l}\text { Time to Reach } 100 \% \text { of LFL } \\
\text { (days) }\end{array}$ \\
\hline 241-SX-105 & 741 & not occur \\
\hline 241-SX-106 & not occur & not occur \\
\hline 241-SX-107 & not occur & not occur \\
\hline $241-S X-108$ & not occur & not occur \\
\hline 241-SX-109 & not occur & not occur \\
\hline 241-SX-110 & not occur & not occur \\
\hline 241-SX-111 & not occur & not occur \\
\hline $241-\mathrm{SX}-112$ & not occur & not occur \\
\hline $241-\mathrm{SX}-113$ & not occur & not occur \\
\hline 241-SX-114 & not occur & not occur \\
\hline $241-S X-115$ & not occur & not occur \\
\hline $241-\mathrm{T}-101$ & $>1826$ & $>1826$ \\
\hline $241-\mathrm{T}-102$ & 1226 & $>1826$ \\
\hline $241-\mathrm{T}-103$ & $>1826$ & $>1826$ \\
\hline 241-T-104 & 1338 & $>1826$ \\
\hline 241-T-105 & $>1826$ & $>1826$ \\
\hline $241-T-106$ & $>1826$ & $>1826$ \\
\hline 241-T-107 & 1361 & $>1826$ \\
\hline 241-T-108 & $>1826$ & $>1826$ \\
\hline 241-T-109 & $>1826$ & $>1826$ \\
\hline 241-T-110 & 475 & $>1826$ \\
\hline 241-T-111 & 971 & $>1826$ \\
\hline 241-T-112 & $>1826$ & $>1826$ \\
\hline 241-T-201 & 166 & 722 \\
\hline 241-T-202 & 737 & $>1826$ \\
\hline 241-T-203 & 291 & 1287 \\
\hline 241-T-204 & 290 & 1283 \\
\hline 241-TX-101 & $>1826$ & $>1826$ \\
\hline 241-TX-102 & $>1826$ & $>1826$ \\
\hline 241-TX-103 & $>1826$ & $>1826$ \\
\hline 241-TX-104 & $>1826$ & $>1826$ \\
\hline 241-TX-105 & 429 & $>1826$ \\
\hline 241-TX-106 & 1226 & $>1826$ \\
\hline 241-TX-107 & $>1826$ & $>1826$ \\
\hline 241-TX-108 & $>1826$ & $>1826$ \\
\hline 241-TX-109 & 915 & $>1826$ \\
\hline 241-TX-110 & 841 & $>1826$ \\
\hline 241-TX-111 & 1166 & $>1826$ \\
\hline $241-\mathrm{TX}-112$ & 551 & $>1826$ \\
\hline 241-TX-113 & 701 & $>1826$ \\
\hline 241-TX-114 & 927 & $>1826$ \\
\hline 241-TX-115 & 716 & $>1826$ \\
\hline $241-T X-116$ & 878 & $>1826$ \\
\hline 241-TX-117 & 1201 & $>1826$ \\
\hline 241-TX-118 & 922 & $>1826$ \\
\hline 241-TY-101 & $>1826$ & $>1826$ \\
\hline 241-TY-102 & $>1826$ & $>1826$ \\
\hline $241-T Y-103$ & $>1826$ & $>1826$ \\
\hline 241-TY-104 & $>1826$ & $>1826$ \\
\hline 241-TY-105 & 1307 & $>1826$ \\
\hline 241-TY-106 & $>1826$ & $>1826$ \\
\hline
\end{tabular}


Table 7-5. Single-Shell Tank Time to Lower Flammability Limit Under Zero Ventilation.

\begin{tabular}{|c|c|c|}
\hline Tank & $\begin{array}{l}\text { Time to Reach } 25 \% \text { of LFL } \\
\text { (days) }\end{array}$ & $\begin{array}{c}\text { Time to Reach } 100 \% \text { of LFL } \\
\text { (days) }\end{array}$ \\
\hline 241-U-101 & $>1826$ & $>1826$ \\
\hline 241-U-102 & 487 & $>1826$ \\
\hline 241-U-103 & 275 & 1251 \\
\hline 241-U-104 & $>1826$ & $>1826$ \\
\hline 241-U-105 & 254 & 1133 \\
\hline 241-U-106 & 657 & $>1826$ \\
\hline 241-U-107 & 982 & $>1826$ \\
\hline 241-U-108 & 429 & $>1826$ \\
\hline 241-U-109 & 528 & $>1826$ \\
\hline $241-\mathrm{U}-110$ & 1253 & $>1826$ \\
\hline 241-U-111 & 1193 & $>1826$ \\
\hline 241-U-112 & $>1826$ & $>1826$ \\
\hline 241-U-201 & $>1826$ & $>1826$ \\
\hline 241-U-202 & $>1826$ & $>1826$ \\
\hline 241-U-203 & $>1826$ & $>1826$ \\
\hline 241-U-204 & 951 & $>1826$ \\
\hline
\end{tabular}

not occur $=$ Gaseous molecular diffusion through the tank domes prevents significant build up of flammable gases in AX and SX tanks as documented in RPP-18491.

Table 7-6. Single-Shell Tank Hydrogen Generation Rate and Minimum Ventilation Rate Required to Maintain Headspace Flammable Gas Concentration Below Specified Level.

\begin{tabular}{|c|c|c|c|}
\hline Tank & $\begin{array}{c}\text { Total HGR } \\
\left(\mathrm{ft}^{\mathbf{3}} / \mathrm{day}\right)\end{array}$ & $\begin{array}{c}\text { Ventilation rate required to } \\
\text { stay below } 25 \% \text { of } \mathrm{LFL} \\
\left(\mathrm{ft}^{3} / \mathrm{min}\right)\end{array}$ & $\begin{array}{c}\text { Ventilation rate required to } \\
\text { stay below } 100 \% \text { of } \mathrm{LFL} \\
\left(\mathrm{ft}^{3} / \mathrm{min}\right)\end{array}$ \\
\hline $241-\mathrm{A}-101$ & 1.6 & 0.12 & 0.029 \\
\hline $241-\mathrm{A}-102$ & 0.56 & 0.044 & 0.010 \\
\hline $241-\mathrm{A}-103$ & 2.6 & 0.21 & 0.048 \\
\hline $241-A-104$ & 0.32 & 0.024 & 0.0057 \\
\hline $241-A-105$ & 0.33 & 0.025 & 0.0058 \\
\hline $241-A-106$ & 3.3 & 0.27 & 0.063 \\
\hline $241-\mathrm{AX}-101$ & 2.0 & 0.16 & 0.037 \\
\hline $241-A X-102$ & 0.74 & 0.058 & 0.014 \\
\hline $241-A X-103$ & 1.2 & 0.094 & 0.022 \\
\hline $241-A X-104$ & 1.05 & 0.083 & 0.020 \\
\hline 241-B-101 & 0.54 & 0.042 & 0.010 \\
\hline 241-B-102 & 0.33 & 0.025 & 0.0058 \\
\hline 241-B-103 & 0.34 & 0.026 & 0.0060 \\
\hline 241-B-104 & 0.51 & 0.039 & 0.0091 \\
\hline 241-B-105 & 0.46 & 0.035 & 0.0081 \\
\hline 241-B-106 & 0.42 & 0.032 & 0.0076 \\
\hline 241-B-107 & 0.40 & 0.030 & 0.0071 \\
\hline 241-B-108 & 0.36 & 0.027 & 0.0064 \\
\hline 241-B-109 & 0.37 & 0.028 & 0.0066 \\
\hline 241-B-110 & 0.72 & 0.056 & 0.013 \\
\hline 241-B-111 & 1.3 & 0.10 & 0.024 \\
\hline 241-B-112 & 0.37 & 0.028 & 0.0067 \\
\hline 241-B-201 & 0.086 & 0.0065 & 0.0015 \\
\hline 241-B-202 & 0.081 & 0.0061 & 0.0014 \\
\hline 241-B-203 & 0.30 & 0.022 & 0.0052 \\
\hline
\end{tabular}


Table 7-6. Single-Shell Tank Hydrogen Generation Rate and Minimum Ventilation Rate Required to Maintain Headspace Flammable Gas Concentration Below Specified Level.

\begin{tabular}{|c|c|c|c|}
\hline Tank & $\begin{array}{c}\text { Total HGR } \\
\left(\mathrm{ft}^{3} / \mathrm{day}\right)\end{array}$ & $\begin{array}{c}\text { Ventilation rate required to } \\
\text { stay below } 25 \% \text { of } \mathrm{LFL} \\
\left(\mathrm{ft}^{\mathbf{3}} / \mathrm{min}\right)\end{array}$ & $\begin{array}{c}\text { Ventilation rate required to } \\
\text { stay below } 100 \% \text { of LFL } \\
\left(\mathrm{ft}^{3} / \mathrm{min}\right)\end{array}$ \\
\hline 241-B-204 & 0.29 & 0.022 & 0.0052 \\
\hline $241-\mathrm{BX}-101$ & 0.37 & 0.029 & 0.0067 \\
\hline 241-BX-102 & 0.49 & 0.038 & 0.0090 \\
\hline 241-BX-103 & 1.07 & 0.085 & 0.020 \\
\hline 241-BX-104 & 0.51 & 0.040 & 0.0093 \\
\hline 241-BX-105 & 0.40 & 0.031 & 0.0072 \\
\hline 241-BX-106 & 0.46 & 0.036 & 0.0083 \\
\hline 241-BX-107 & 0.58 & 0.044 & 0.010 \\
\hline 241-BX-108 & 0.34 & 0.026 & 0.0061 \\
\hline 241-BX-109 & 0.69 & 0.053 & 0.013 \\
\hline $241-\mathrm{BX}-110$ & 0.54 & 0.042 & 0.010 \\
\hline 241-BX-111 & 0.44 & 0.034 & 0.0079 \\
\hline $241-\mathrm{BX}-112$ & 0.47 & 0.036 & 0.0084 \\
\hline 241-BY-101 & 1.1 & 0.086 & 0.020 \\
\hline 241-BY-102 & 0.53 & 0.040 & 0.0094 \\
\hline 241-BY-103 & 0.65 & 0.050 & 0.012 \\
\hline 241-BY-104 & 1.5 & 0.12 & 0.028 \\
\hline 241-BY-105 & 0.88 & 0.068 & 0.016 \\
\hline 241-BY-106 & 1.2 & 0.099 & 0.023 \\
\hline 241-BY-107 & 0.79 & 0.062 & 0.015 \\
\hline 241-BY-108 & 0.78 & 0.061 & 0.014 \\
\hline 241-BY-109 & 0.54 & 0.042 & 0.010 \\
\hline 241-BY-110 & 2.6 & 0.21 & 0.048 \\
\hline 241-BY-111 & 0.65 & 0.050 & 0.012 \\
\hline 241-BY-112 & 0.59 & 0.045 & 0.011 \\
\hline 241-C-101 & 0.44 & 0.034 & 0.0079 \\
\hline $241-C-102$ & 0.79 & 0.061 & 0.014 \\
\hline 241-C-103 & 0.81 & 0.061 & 0.014 \\
\hline 241-C-104 & 2.0 & 0.16 & 0.038 \\
\hline 241-C-105 & 1.0 & 0.078 & 0.018 \\
\hline $241-\mathrm{C}-106$ & 1.34 & 0.10 & 0.025 \\
\hline $241-C-107$ & 5.3 & 0.43 & 0.10 \\
\hline $241-C-108$ & 0.81 & 0.061 & 0.014 \\
\hline 241-C-109 & 0.32 & 0.024 & 0.0056 \\
\hline $241-C-110$ & 0.33 & 0.025 & 0.0059 \\
\hline 241-C-111 & 1.6 & 0.13 & 0.031 \\
\hline $241-\mathrm{C}-112$ & 1.8 & 0.15 & 0.034 \\
\hline $241-C-201$ & 0.063 & 0.0048 & 0.0011 \\
\hline $241-\mathrm{C}-202$ & 0.064 & 0.0048 & 0.0011 \\
\hline $241-C-203$ & 0.061 & 0.0046 & 0.0011 \\
\hline $241-C-204$ & 0.061 & 0.0046 & 0.0011 \\
\hline $241-S-101$ & 1.3 & 0.10 & 0.024 \\
\hline $241-S-102$ & 0.37 & 0.028 & 0.0067 \\
\hline $241-S-103$ & 0.76 & 0.060 & 0.014 \\
\hline $241-S-104$ & 0.81 & 0.063 & 0.015 \\
\hline 241-S-105 & 0.55 & 0.042 & 0.010 \\
\hline 241-S-106 & 0.74 & 0.057 & 0.013 \\
\hline $241-S-107$ & 0.97 & 0.076 & 0.018 \\
\hline $241-S-108$ & 0.6 & 0.046 & 0.011 \\
\hline
\end{tabular}


Table 7-6. Single-Shell Tank Hydrogen Generation Rate and Minimum Ventilation Rate Required to Maintain Headspace Flammable Gas Concentration Below Specified Level.

\begin{tabular}{|c|c|c|c|}
\hline Tank & $\begin{array}{c}\text { Total HGR } \\
\left(\mathrm{ft}^{3} / \text { day }\right)\end{array}$ & $\begin{array}{c}\text { Ventilation rate required to } \\
\text { stay below } 25 \% \text { of } \mathrm{LFL} \\
\left(\mathrm{ft}^{\mathbf{3}} / \mathrm{min}\right)\end{array}$ & $\begin{array}{c}\begin{array}{c}\text { Ventilation rate required to } \\
\text { stay below } 100 \% \text { of } L F L \\
\left(\mathrm{ft}^{3} / \mathrm{min}\right)\end{array} \\
\end{array}$ \\
\hline 241-S-109 & 0.61 & 0.046 & 0.011 \\
\hline $241-S-110$ & 0.90 & 0.068 & 0.016 \\
\hline $241-S-111$ & 0.87 & 0.068 & 0.016 \\
\hline 241-S-112 & 0.78 & 0.059 & 0.014 \\
\hline 241-SX-101 & 0.99 & 0.078 & 0.018 \\
\hline 241-SX-102 & 2.3 & 0.19 & 0.043 \\
\hline 241-SX-103 & 8.8 & 0.71 & 0.17 \\
\hline 241-SX-104 & 3.7 & 0.30 & 0.069 \\
\hline 241-SX-105 & 7.8 & 0.64 & 0.15 \\
\hline 241-SX-106 & 1.1 & 0.086 & 0.020 \\
\hline 241-SX-107 & 2.2 & 0.18 & 0.042 \\
\hline 241-SX-108 & 0.73 & 0.058 & 0.014 \\
\hline 241-SX-109 & 7.0 & 0.56 & 0.13 \\
\hline 241-SX-110 & 3.7 & 0.30 & 0.070 \\
\hline 241-SX-111 & 4.5 & 0.37 & 0.087 \\
\hline $241-\mathrm{SX}-112$ & 1.4 & 0.11 & 0.026 \\
\hline $241-\mathrm{SX}-113$ & 0.33 & 0.025 & 0.0059 \\
\hline 241-SX-114 & 4.5 & 0.37 & 0.086 \\
\hline 241-SX-115 & 0.47 & 0.037 & 0.0086 \\
\hline $241-\mathrm{T}-101$ & 0.42 & 0.032 & 0.0074 \\
\hline $241-\mathrm{T}-102$ & 0.84 & 0.064 & 0.015 \\
\hline $241-\mathrm{T}-103$ & 0.33 & 0.025 & 0.0059 \\
\hline $241-T-104$ & 0.51 & 0.039 & 0.0091 \\
\hline 241-T-105 & 0.44 & 0.034 & 0.0079 \\
\hline $241-T-106$ & 0.32 & 0.024 & 0.0057 \\
\hline $241-\mathrm{T}-107$ & 0.62 & 0.048 & 0.011 \\
\hline $241-T-108$ & 0.32 & 0.024 & 0.0056 \\
\hline $241-\mathrm{T}-109$ & 0.34 & 0.026 & 0.0061 \\
\hline $241-T-110$ & 1.2 & 0.094 & 0.022 \\
\hline $241-T-111$ & 0.53 & 0.040 & 0.009 \\
\hline $241-T-112$ & 0.35 & 0.027 & 0.0062 \\
\hline $241-\mathrm{T}-201$ & 0.21 & 0.016 & 0.0037 \\
\hline $241-T-202$ & 0.064 & 0.0049 & 0.0011 \\
\hline $241-T-203$ & 0.095 & 0.0072 & 0.0017 \\
\hline $241-\mathrm{T}-204$ & 0.095 & 0.0072 & 0.0017 \\
\hline 241-TX-101 & 0.51 & 0.039 & 0.0092 \\
\hline 241-TX-102 & 0.56 & 0.044 & 0.010 \\
\hline 241-TX-103 & 0.46 & 0.035 & 0.0082 \\
\hline 241-TX-104 & 0.43 & 0.033 & 0.0078 \\
\hline 241-TX-105 & 1.3 & 0.102 & 0.024 \\
\hline 241-TX-106 & 0.71 & 0.055 & 0.013 \\
\hline 241-TX-107 & 0.34 & 0.026 & 0.0061 \\
\hline 241-TX-108 & 0.43 & 0.033 & 0.0077 \\
\hline 241-TX-109 & 0.91 & 0.071 & 0.017 \\
\hline 241-TX-110 & 0.84 & 0.065 & 0.015 \\
\hline 241-TX-111 & 0.72 & 0.056 & 0.013 \\
\hline 241-TX-112 & 0.91 & 0.071 & 0.017 \\
\hline 241-TX-113 & 0.73 & 0.055 & 0.013 \\
\hline 241-TX-114 & 0.69 & 0.053 & 0.012 \\
\hline
\end{tabular}


Table 7-6. Single-Shell Tank Hydrogen Generation Rate and Minimum Ventilation Rate Required to Maintain Headspace Flammable Gas Concentration Below Specified Level.

\begin{tabular}{|c|c|c|c|}
\hline Tank & $\begin{array}{c}\text { Total HGR } \\
\left(\mathbf{f t}^{\mathbf{3}} \text { /day) }\right.\end{array}$ & $\begin{array}{c}\text { Ventilation rate required to } \\
\text { stay below 25\% of LFL } \\
\left(\mathbf{f t}^{\mathbf{3}} / \mathbf{m i n}\right)\end{array}$ & $\begin{array}{c}\text { Ventilation rate required to } \\
\text { stay below 100\% of LFL } \\
\left(\mathbf{f t}^{\mathbf{3}} / \mathbf{m i n}\right)\end{array}$ \\
\hline $241-\mathrm{TX}-115$ & 0.84 & 0.065 & $\mathbf{0 . 0 1 5}$ \\
\hline $241-\mathrm{TX}-116$ & 0.65 & 0.049 & 0.011 \\
\hline $241-\mathrm{TX}-117$ & 0.6 & 0.05 & 0.011 \\
\hline $241-\mathrm{TX}-118$ & 1.0 & 0.082 & $\mathbf{0 . 0 1 9}$ \\
\hline $241-\mathrm{TY}-101$ & 0.38 & 0.029 & 0.0067 \\
\hline $241-\mathrm{TY}-102$ & 0.36 & 0.027 & 0.0063 \\
\hline $241-\mathrm{TY}-103$ & 0.55 & 0.043 & 0.010 \\
\hline $241-\mathrm{TY}-104$ & 0.39 & 0.030 & 0.0070 \\
\hline $241-\mathrm{TY}-105$ & 0.77 & 0.060 & 0.014 \\
\hline $241-\mathrm{TY}-106$ & 0.34 & 0.026 & 0.0061 \\
\hline $241-\mathrm{U}-101$ & 0.40 & 0.030 & 0.0071 \\
\hline $241-\mathrm{U}-102$ & 1.3 & 0.100 & 0.024 \\
\hline $241-\mathrm{U}-103$ & 1.5 & 0.12 & 0.028 \\
\hline $241-\mathrm{U}-104$ & 0.40 & 0.031 & 0.0072 \\
\hline $241-\mathrm{U}-105$ & 2.2 & 0.18 & 0.042 \\
\hline $241-\mathrm{U}-106$ & 1.2 & 0.10 & 0.023 \\
\hline $241-\mathrm{U}-107$ & 0.70 & 0.054 & 0.013 \\
\hline $241-\mathrm{U}-108$ & 1.1 & 0.090 & 0.021 \\
\hline $241-\mathrm{U}-109$ & 0.83 & 0.065 & 0.015 \\
\hline $241-\mathrm{U}-110$ & 0.66 & 0.052 & 0.012 \\
\hline $241-\mathrm{U}-111$ & 0.65 & 0.051 & 0.012 \\
\hline $241-\mathrm{U}-112$ & 0.41 & 0.031 & 0.0073 \\
\hline $241-\mathrm{U}-201$ & 0.031 & 0.0024 & 0.0006 \\
\hline $241-\mathrm{U}-202$ & 0.031 & 0.0023 & 0.0005 \\
\hline $241-\mathrm{U}-203$ & 0.030 & 0.0023 & 0.0005 \\
\hline $241-\mathrm{U}-204$ & 0.073 & 0.0055 & 0.0013 \\
\hline
\end{tabular}




\subsection{CONCLUSIONS}

Flammable gas concentrations, in terms of percent of the LFL in the tank headspace, were evaluated under off-normal conditions for all 177 tanks. Assuming barometric breathing only, the shortest time to reach $25 \%$ of the LFL is 36 days for SST $241-\mathrm{B}-203$ and 11 days for DST 241-AZ-101. Assuming zero ventilation, the shortest time to reach $25 \%$ of the LFL is 34 days for SST 241-B-203 and 10 days for DST 241-AZ-101.

The steady-state flammability level in the tank headspace was evaluated based on the steady-state flammable gas concentrations and Le Chatelier's principle. Overall, the calculations under off-normal conditions show that 23 DSTs and 7 SSTs will reach $25 \%$ of the LFL and 7 DSTs and 2 SSTs will reach $100 \%$ of the LFL if allowed to reach steady-state under barometric breathing only.

Results can be used to establish technical safety requirements for ventilation in tanks, to establish maximum permissible down times for active ventilation systems, to develop procedures for operations that require closing vent valves to HEPA filters on passively ventilated tanks, and to support the technical basis for flammable gas control strategies.

\subsection{REFERENCES}

Coward, H. F., and G. W. Jones, 1952, Limits of Flammability of Gases and Vapors, Bureau of Mines Bulletin 503, U.S. Bureau of Mines, Washington, D.C..

HNF-3851, 2004, Empirical Rate Equation Model and Rate Calculations of Hydrogen Generation for Hanford Tank Waste, Rev. 1, CH2M HILL Hanford Group, Inc., Richland, Washington.

HNF-EP-0063, 2008, Hanford Site Solid Waste Acceptance Criteria, Rev. 14 (reissue), Fluor Hanford, Richland, Washington.

Pfahl, U., and J. Shepherd, 1997, Flammability and Flame Propagation in $\mathrm{H}_{2}-\mathrm{N}_{2} \mathrm{O}-\mathrm{CH}_{4}-\mathrm{NH}_{3}-\mathrm{O}_{2}-\mathrm{N}_{2}$ Mixtures, Explosion Dynamics Laboratory Report FM97-4, Graduate Aeronautical Laboratories, California Institute of Technology, Pasadena, California.

PNNL-12181, 1999, Thermal and Radiolytic Gas Generation Tests on Material from Tanks 241-U-103, 241-AW-101, 241-S-106, and 241-S-102: Status Report, Pacific Northwest National Laboratory, Richland, Washington.

PNNL-13000, 1999, Retained Gas Sampling Results for the Flammable Gas Program, Rev. 0, Pacific Northwest National Laboratory, Richland, Washington.

RPP-13019, 2003, Determination of Hanford Waste Tank Volumes, Rev. 0, CH2M HILL Hanford Group, Inc., Richland, Washington. 
RPP-18491, 2003, Flammable Gas Diffusion Through Single-Shell Tank Domes, Rev. 0, CH2M HILL Hanford Group, Inc., Richland, Washington.

Surveillance Analysis Computer System (SACS), Queried October 1, 2009, [Tank temperature data October 1, 2008 through October 1, 2009], HISI ID No. 242, http://www7.rl.gov/hisiconsol/Form/AppUpdate.cfm?AppID=242\&WasSearch=Y.

SNL-000198, 1999, Flammable Gas Safety Analysis Data Review, Sandia National Laboratory, Albuquerque, New Mexico.

SVF-1536, 2009, DST_and_SST_TTLFL_v4.xlsm, Version 4, Rev. 3, Washington River Protection Solutions, Richland, Washington.

SVF-1570, 2009, Raw Input Data for RPP-5926_v2.xls, Version 2, Rev. 1, Washington River Protection Solutions, Richland, Washington.

Tank Waste Information Network System (TWINS), Queried October 1, 2009, [Best Basis Inventory data], internet address: http://twins.pnl.gov/twins.htm.

WHC-EP-0651, 1993, Barometric Pressure Variations, Westinghouse Hanford Company, Richland, Washington.

WHC-SD-WM-ES-219, 1992, Laboratory Flammability Studies of Mixtures of Hydrogen, Nitrous Oxide, and Air, Rev. 0, Westinghouse Hanford Company, Richland, Washington. 
RPP-5926 REV 9

APPENDIX A

INPUT DATA FOR TIME TO THE LOWER FLAMMABILITY LIMIT CALCULATIONS 
Table A-1. Tank Waste Chemical Concentrations.

\begin{tabular}{|c|c|c|c|c|c|c|c|}
\hline Tank & Layer & $\begin{array}{c}\mathbf{O H} \\
(\mu \mathrm{g} / \mathrm{mL})\end{array}$ & $\begin{array}{c}\text { TOC } \\
(\mu \mathrm{g} / \mathrm{mL})\end{array}$ & $\begin{array}{c}\mathrm{NO}_{2}^{-} \\
(\mu \mathrm{g} / \mathrm{mL})\end{array}$ & $\begin{array}{c}\mathrm{NO}_{3}^{-} \\
(\mu \mathrm{g} / \mathrm{mL})\end{array}$ & $\begin{array}{c}\mathrm{Na}^{+} \\
(\mu \mathrm{g} / \mathrm{mL})\end{array}$ & $\begin{array}{c}\mathrm{Al}^{+3} \\
(\mu \mathrm{g} / \mathrm{mL})\end{array}$ \\
\hline 241-AN-101 & Supernatant & $3.28 \mathrm{E}+04$ & $5.37 \mathrm{E}+03$ & $1.11 \mathrm{E}+05$ & $1.62 \mathrm{E}+05$ & $2.10 \mathrm{E}+05$ & $1.91 \mathrm{E}+04$ \\
\hline 241-AN-101 & Solid & $4.81 \mathrm{E}+04$ & $4.04 \mathrm{E}+03$ & $1.22 \mathrm{E}+05$ & $1.90 \mathrm{E}+05$ & $2.36 \mathrm{E}+05$ & $3.79 E+04$ \\
\hline $241-\mathrm{AN}-102$ & Supernatant & $8.18 \mathrm{E}+03$ & $2.41 \mathrm{E}+04$ & $8.71 \mathrm{E}+04$ & $1.97 \mathrm{E}+05$ & $2.12 \mathrm{E}+05$ & $1.32 \mathrm{E}+04$ \\
\hline 241-AN-102 & Solid & $8.18 \mathrm{E}+03$ & $2.41 \mathrm{E}+04$ & $8.71 E+04$ & $1.97 \mathrm{E}+05$ & $2.12 E+05$ & $1.32 \mathrm{E}+04$ \\
\hline $241-\mathrm{AN}-103$ & Supernatant & $6.73 E+04$ & $3.05 \mathrm{E}+03$ & $1.30 \mathrm{E}+05$ & $1.28 \mathrm{E}+05$ & $2.70 \mathrm{E}+05$ & $3.00 \mathrm{E}+04$ \\
\hline $241-\mathrm{AN}-103$ & Solid & $6.73 \mathrm{E}+04$ & $3.05 \mathrm{E}+03$ & $1.30 \mathrm{E}+05$ & $1.28 \mathrm{E}+05$ & $2.70 \mathrm{E}+05$ & $3.00 \mathrm{E}+04$ \\
\hline 241-AN-104 & Supernatant & $6.53 \mathrm{E}+04$ & $3.14 \mathrm{E}+03$ & $1.19 \mathrm{E}+05$ & $1.89 \mathrm{E}+05$ & $2.58 \mathrm{E}+05$ & $3.90 \mathrm{E}+04$ \\
\hline $241-\mathrm{AN}-104$ & Solid & $6.53 \mathrm{E}+04$ & $3.14 \mathrm{E}+03$ & $1.19 \mathrm{E}+05$ & $1.89 \mathrm{E}+05$ & $2.58 \mathrm{E}+05$ & $3.90 \mathrm{E}+04$ \\
\hline 241-AN-105 & Supernatant & $5.98 \mathrm{E}+04$ & $2.76 \mathrm{E}+03$ & $1.19 \mathrm{E}+05$ & $1.56 \mathrm{E}+05$ & $2.47 \mathrm{E}+05$ & $4.17 \mathrm{E}+04$ \\
\hline $241-\mathrm{AN}-105$ & Solid & $5.98 \mathrm{E}+04$ & $2.76 \mathrm{E}+03$ & $1.19 \mathrm{E}+05$ & $1.56 \mathrm{E}+05$ & $2.47 \mathrm{E}+05$ & $4.17 E+04$ \\
\hline 241-AN-106 & Supernatant & $1.46 \mathrm{E}+02$ & $1.12 \mathrm{E}+03$ & $2.73 \mathrm{E}+04$ & $4.03 \mathrm{E}+04$ & $8.39 \mathrm{E}+04$ & $2.79 \mathrm{E}+03$ \\
\hline 241-AN-106 & Solid & $1.46 \mathrm{E}+02$ & $1.12 \mathrm{E}+03$ & $2.73 \mathrm{E}+04$ & $4.03 \mathrm{E}+04$ & $8.39 \mathrm{E}+04$ & $2.79 \mathrm{E}+03$ \\
\hline $241-\mathrm{AN}-107$ & Supernatant & $1.80 \mathrm{E}+04$ & $3.25 \mathrm{E}+04$ & $6.67 \mathrm{E}+04$ & $2.09 E+05$ & $2.08 \mathrm{E}+05$ & $1.12 \mathrm{E}+03$ \\
\hline $241-\mathrm{AN}-107$ & Solid & $1.80 \mathrm{E}+04$ & $3.25 E+04$ & $6.67 \mathrm{E}+04$ & $2.09 \mathrm{E}+05$ & $2.08 \mathrm{E}+05$ & $1.12 \mathrm{E}+03$ \\
\hline 241-AP-101 & Supernatant & $1.79 E+04$ & $9.97 \mathrm{E}+02$ & $2.10 \mathrm{E}+04$ & $1.71 \mathrm{E}+05$ & $1.11 \mathrm{E}+05$ & $5.80 \mathrm{E}+03$ \\
\hline 241-AP-101 & Solid & $1.79 E+04$ & $9.97 \mathrm{E}+02$ & $2.10 \mathrm{E}+04$ & $1.71 \mathrm{E}+05$ & $1.11 \mathrm{E}+05$ & $5.80 \mathrm{E}+03$ \\
\hline 241-AP-102 & Supernatant & $3.42 \mathrm{E}+04$ & $2.38 \mathrm{E}+03$ & $9.57 \mathrm{E}+04$ & $1.67 \mathrm{E}+05$ & $1.85 \mathrm{E}+05$ & $2.42 \mathrm{E}+04$ \\
\hline 241-AP-102 & Solid & $3.42 \mathrm{E}+04$ & $2.38 \mathrm{E}+03$ & $9.57 \mathrm{E}+04$ & $1.67 \mathrm{E}+05$ & $1.85 \mathrm{E}+05$ & $2.42 \mathrm{E}+04$ \\
\hline 241-AP-103 & Supernatant & $1.73 E+04$ & $4.91 \mathrm{E}+03$ & $9.10 \mathrm{E}+04$ & $1.68 \mathrm{E}+05$ & $1.87 \mathrm{E}+\mathbf{0 5}$ & $1.84 \mathrm{E}+04$ \\
\hline 241-AP-103 & Solid & $1.73 E+04$ & $4.91 \mathrm{E}+03$ & $9.10 \mathrm{E}+04$ & $1.68 \mathrm{E}+05$ & $1.87 \mathrm{E}+05$ & $1.84 \mathrm{E}+04$ \\
\hline 241-AP-104 & Supernatant & $2.69 \mathrm{E}+04$ & $6.76 E+03$ & $9.50 \mathrm{E}+04$ & $1.43 \mathrm{E}+05$ & $1.92 \mathrm{E}+05$ & $2.34 \mathrm{E}+04$ \\
\hline 241-AP-104 & Solid & $2.69 \mathrm{E}+04$ & $6.76 \mathrm{E}+03$ & $9.50 \mathrm{E}+04$ & $1.43 \mathrm{E}+05$ & $1.92 \mathrm{E}+05$ & $2.34 \mathrm{E}+04$ \\
\hline 241-AP-105 & Supernatant & $1.18 \mathrm{E}+04$ & $1.51 \mathrm{E}+03$ & $3.17 \mathrm{E}+04$ & $1.22 \mathrm{E}+05$ & $1.06 \mathrm{E}+05$ & $6.69 \mathrm{E}+03$ \\
\hline 241-AP-105 & Solid & $1.18 E+04$ & $1.51 \mathrm{E}+03$ & $3.17 \mathrm{E}+04$ & $1.22 \mathrm{E}+05$ & $1.06 \mathrm{E}+05$ & $6.69 \mathrm{E}+03$ \\
\hline 241-AP-106 & Supernatant & $7.36 \mathrm{E}+03$ & $3.46 \mathrm{E}+03$ & $4.13 \mathrm{E}+04$ & $7.06 \mathrm{E}+04$ & $1.04 \mathrm{E}+05$ & $1.35 \mathrm{E}+04$ \\
\hline 241-AP-106 & Solid & $7.36 \mathrm{E}+03$ & $3.46 \mathrm{E}+03$ & $4.13 \mathrm{E}+04$ & $7.06 \mathrm{E}+04$ & $1.04 \mathrm{E}+05$ & $1.35 \mathrm{E}+04$ \\
\hline 241-AP-107 & Supernatant & $1.37 \mathrm{E}+04$ & $1.46 \mathrm{E}+03$ & $3.35 \mathrm{E}+04$ & $1.10 \mathrm{E}+05$ & $1.06 \mathrm{E}+05$ & $6.53 \mathrm{E}+03$ \\
\hline 241-AP-107 & Solid & $1.37 \mathrm{E}+04$ & $1.46 \mathrm{E}+03$ & $3.35 \mathrm{E}+04$ & $1.10 \mathrm{E}+05$ & $1.06 \mathrm{E}+05$ & $6.53 \mathrm{E}+03$ \\
\hline 241-AP-108 & Supernatant & $3.82 \mathrm{E}+04$ & $2.89 \mathrm{E}+03$ & $8.69 \mathrm{E}+04$ & $1.95 \mathrm{E}+05$ & $2.01 \mathrm{E}+05$ & $2.30 \mathrm{E}+04$ \\
\hline 241-AP-108 & Solid & $3.82 \mathrm{E}+04$ & $2.89 E+03$ & $8.69 \mathrm{E}+04$ & $1.95 \mathrm{E}+05$ & $2.01 \mathrm{E}+05$ & $2.30 \mathrm{E}+04$ \\
\hline 241-AW-101 & Supernatant & $9.95 E+04$ & $2.61 \mathrm{E}+03$ & $1.05 E+05$ & $1.71 E+05$ & $2.33 E+05$ & $2.95 \mathrm{E}+04$ \\
\hline $241-\mathrm{AW}-101$ & Solid & $9.95 \mathrm{E}+04$ & $2.61 \mathrm{E}+03$ & $1.05 \mathrm{E}+05$ & $1.71 \mathrm{E}+05$ & $2.33 \mathrm{E}+05$ & $2.95 \mathrm{E}+04$ \\
\hline $241-\mathrm{AW}-102$ & Supernatant & $1.56 \mathrm{E}+04$ & $1.04 \mathrm{E}+03$ & $2.08 \mathrm{E}+04$ & $1.59 \mathrm{E}+05$ & $1.04 \mathrm{E}+05$ & $5.82 \mathrm{E}+03$ \\
\hline 241-AW-102 & Solid & $1.56 \mathrm{E}+04$ & $1.04 E+03$ & $2.08 \mathrm{E}+04$ & $1.59 \mathrm{E}+05$ & $1.04 \mathrm{E}+05$ & $5.82 \mathrm{E}+03$ \\
\hline 241-AW-103 & Supernatant & $1.31 \mathrm{E}+04$ & $1.88 \mathrm{E}+03$ & $3.36 \mathrm{E}+04$ & $9.66 \mathrm{E}+04$ & $1.01 \mathrm{E}+05$ & $8.12 \mathrm{E}+03$ \\
\hline 241-AW-103 & Solid & $2.72 \mathrm{E}+04$ & $6.11 \mathrm{E}+03$ & $9.31 \mathrm{E}+04$ & $2.47 \mathrm{E}+05$ & $2.01 \mathrm{E}+05$ & $8.08 \mathrm{E}+03$ \\
\hline 241-AW-104 & Supernatant & $2.56 \mathrm{E}+04$ & $4.33 \mathrm{E}+03$ & $7.52 \mathrm{E}+04$ & $1.14 \mathrm{E}+05$ & $1.72 E+05$ & $2.58 \mathrm{E}+04$ \\
\hline 241-AW-104 & Solid & $3.63 \mathrm{E}+04$ & $3.26 \mathrm{E}+03$ & $7.33 \mathrm{E}+04$ & $1.08 \mathrm{E}+05$ & $2.26 \mathrm{E}+05$ & $2.91 \mathrm{E}+04$ \\
\hline 241-AW-105 & Supernatant & $4.81 \mathrm{E}+03$ & $5.56 \mathrm{E}+02$ & $3.06 \mathrm{E}+03$ & $2.73 \mathrm{E}+04$ & $2.30 \mathrm{E}+04$ & $1.90 \mathrm{E}+02$ \\
\hline $241-\mathrm{AW}-105$ & Solid & $4.81 \mathrm{E}+03$ & $5.56 \mathrm{E}+02$ & $3.06 \mathrm{E}+03$ & $2.73 E+04$ & $2.30 \mathrm{E}+04$ & $1.90 \mathrm{E}+02$ \\
\hline 241-AW-106 & Supernatant & $1.00 \mathrm{E}+04$ & $2.12 \mathrm{E}+03$ & $3.36 \mathrm{E}+04$ & $1.07 \mathrm{E}+05$ & $1.09 \mathrm{E}+05$ & $8.12 E+03$ \\
\hline 241-AW-106 & Solid & $1.00 \mathrm{E}+04$ & $2.12 \mathrm{E}+03$ & $3.36 \mathrm{E}+04$ & $1.07 \mathrm{E}+05$ & $1.09 \mathrm{E}+05$ & $8.12 \mathrm{E}+03$ \\
\hline 241-AY-101 & Supernatant & $7.57 \mathrm{E}+03$ & $5.92 \mathrm{E}+02$ & $8.94 \mathrm{E}+03$ & $3.67 \mathrm{E}+04$ & $3.88 \mathrm{E}+04$ & $1.99 \mathrm{E}+03$ \\
\hline 241-AY-101 & Solid & $3.75 \mathrm{E}+02$ & $1.03 \mathrm{E}+03$ & $3.93 \mathrm{E}+04$ & $3.77 \mathrm{E}+03$ & $1.27 \mathrm{E}+05$ & $8.63 \mathrm{E}+02$ \\
\hline $241-\mathrm{AY}-102$ & Supernatant & $3.93 \mathrm{E}+04$ & $1.92 \mathrm{E}+03$ & $4.23 \mathrm{E}+04$ & $1.15 E+05$ & $1.31 \mathrm{E}+05$ & $7.22 E+03$ \\
\hline 241-AY-102 & Solid & $5.52 \mathrm{E}+02$ & $1.53 \mathrm{E}+03$ & $7.25 \mathrm{E}+03$ & $3.88 \mathrm{E}+02$ & $7.03 \mathrm{E}+04$ & $3.41 \mathrm{E}+02$ \\
\hline 241-AZ-101 & Supernatant & $1.09 \mathrm{E}+04$ & $6.77 \mathrm{E}+02$ & $6.07 \mathrm{E}+04$ & $5.15 \mathrm{E}+04$ & $1.15 \mathrm{E}+05$ & $5.79 E+03$ \\
\hline $241-\mathrm{AZ}-101$ & Solid & $1.09 E+04$ & $6.77 \mathrm{E}+02$ & $6.07 \mathrm{E}+04$ & $5.15 \mathrm{E}+04$ & $1.15 E+05$ & $5.79 \mathrm{E}+03$ \\
\hline 241-AZ-102 & Supernatant & $2.30 \mathrm{E}+03$ & $1.20 \mathrm{E}+03$ & $3.88 \mathrm{E}+04$ & $1.53 \mathrm{E}+04$ & $6.57 \mathrm{E}+04$ & $6.37 \mathrm{E}+02$ \\
\hline $241-\mathrm{AZ}-102$ & Solid & $2.30 \mathrm{E}+03$ & $1.20 \mathrm{E}+03$ & $3.88 \mathrm{E}+04$ & $1.53 \mathrm{E}+04$ & $6.57 \mathrm{E}+04$ & $6.37 \mathrm{E}+02$ \\
\hline
\end{tabular}


Table A-1. Tank Waste Chemical Concentrations.

\begin{tabular}{|c|c|c|c|c|c|c|c|}
\hline Tank & Layer & $\begin{array}{c}\mathbf{O H}^{-} \\
(\mu \mathrm{g} / \mathrm{mL})\end{array}$ & $\begin{array}{c}\text { TOC } \\
(\mu \mathrm{g} / \mathrm{mL})\end{array}$ & $\begin{array}{c}\mathrm{NO}_{2}^{-} \\
(\mu \mathrm{g} / \mathrm{mL})\end{array}$ & $\begin{array}{c}\mathrm{NO}_{3}^{-} \\
(\mu \mathrm{g} / \mathrm{mL})\end{array}$ & $\begin{array}{c}\mathrm{Na}^{+} \\
(\mu \mathrm{g} / \mathrm{mL})\end{array}$ & $\begin{array}{c}\mathrm{Al}^{+3} \\
(\mu \mathrm{g} / \mathrm{mL})\end{array}$ \\
\hline 241-SY-101 & Supernatant & $1.11 \mathrm{E}+04$ & $9.90 \mathrm{E}+02$ & $9.29 E+03$ & $5.76 \mathrm{E}+04$ & $5.63 \mathrm{E}+04$ & $3.78 \mathrm{E}+03$ \\
\hline 241-SY-101 & Solid & $1.11 \mathrm{E}+04$ & $9.90 \mathrm{E}+02$ & $9.29 \mathrm{E}+03$ & $5.76 \mathrm{E}+04$ & $5.63 \mathrm{E}+04$ & $3.78 \mathrm{E}+03$ \\
\hline 241-SY-102 & Supernatant & $8.83 E+03$ & $7.95 E+02$ & $7.73 E+03$ & $4.62 \mathrm{E}+04$ & $4.41 \mathrm{E}+04$ & $2.93 \mathrm{E}+03$ \\
\hline 241-SY-102 & Solid & $2.07 \mathrm{E}+04$ & $3.61 E+03$ & $5.61 \mathrm{E}+04$ & $1.27 \mathrm{E}+05$ & $1.34 \mathrm{E}+05$ & $7.61 \mathrm{E}+03$ \\
\hline $241-S Y-103$ & Supernatant & $3.74 \mathrm{E}+04$ & $6.23 E+03$ & $1.52 \mathrm{E}+05$ & $1.68 \mathrm{E}+05$ & $2.15 E+05$ & $4.07 \mathrm{E}+04$ \\
\hline 241-SY-103 & Solid & $3.74 \mathrm{E}+04$ & $6.23 E+03$ & $1.52 E+05$ & $1.68 \mathrm{E}+05$ & $2.15 E+05$ & $4.07 \mathrm{E}+04$ \\
\hline $241-A-101$ & Supernatant & $4.27 \mathrm{E}+04$ & $0.00 \mathrm{E}+00$ & $0.00 \mathrm{E}+00$ & $0.00 \mathrm{E}+00$ & $0.00 \mathrm{E}+00$ & $0.00 \mathrm{E}+00$ \\
\hline $241-\mathrm{A}-101$ & Solid & $4.27 \mathrm{E}+04$ & $3.74 E+03$ & $1.65 E+05$ & $2.33 E+05$ & $2.78 \mathrm{E}+05$ & $5.18 \mathrm{E}+04$ \\
\hline $241-\mathrm{A}-102$ & Supernatant & $1.73 E+04$ & $4.42 \mathrm{E}+03$ & $1.73 E+05$ & $1.89 \mathrm{E}+05$ & $2.00 \mathrm{E}+05$ & $4.10 \mathrm{E}+04$ \\
\hline $241-A-102$ & Solid & $1.73 E+04$ & $4.42 \mathrm{E}+03$ & $1.72 E+05$ & $1.89 E+05$ & $2.00 \mathrm{E}+05$ & $4.09 \mathrm{E}+04$ \\
\hline $241-A-103$ & Supernatant & $4.89 \mathrm{E}+04$ & $8.29 \mathrm{E}+03$ & $1.24 E+05$ & $2.42 \mathrm{E}+05$ & $2.35 \mathrm{E}+05$ & $3.55 \mathrm{E}+04$ \\
\hline $241-A-103$ & Solid & $4.89 \mathrm{E}+04$ & $8.31 \mathrm{E}+03$ & $1.24 E+05$ & $2.42 \mathrm{E}+05$ & $2.35 E+05$ & $3.55 \mathrm{E}+04$ \\
\hline $241-A-104$ & Supernatant & $0.00 \mathrm{E}+00$ & $0.00 \mathrm{E}+00$ & $0.00 \mathrm{E}+00$ & $0.00 \mathrm{E}+00$ & $0.00 \mathrm{E}+00$ & $0.00 \mathrm{E}+00$ \\
\hline $241-A-104$ & Solid & $4.27 \mathrm{E}+04$ & $2.98 \mathrm{E}+03$ & $3.60 \mathrm{E}+04$ & $7.96 \mathrm{E}+04$ & $8.32 \mathrm{E}+04$ & $4.94 \mathrm{E}+03$ \\
\hline $241-A-105$ & Supernatant & $0.00 \mathrm{E}+00$ & $0.00 \mathrm{E}+00$ & $0.00 \mathrm{E}+00$ & $0.00 \mathrm{E}+00$ & $0.00 \mathrm{E}+00$ & $0.00 \mathrm{E}+00$ \\
\hline $241-A-105$ & Solid & $4.27 \mathrm{E}+04$ & $2.98 \mathrm{E}+03$ & $3.60 \mathrm{E}+04$ & $7.96 \mathrm{E}+04$ & $8.32 \mathrm{E}+04$ & $4.94 \mathrm{E}+03$ \\
\hline $241-A-106$ & Supernatant & $0.00 \mathrm{E}+00$ & $0.00 \mathrm{E}+00$ & $0.00 \mathrm{E}+00$ & $0.00 \mathrm{E}+00$ & $0.00 \mathrm{E}+00$ & $0.00 \mathrm{E}+00$ \\
\hline $241-\mathrm{A}-106$ & Solid & $4.27 \mathrm{E}+04$ & $1.10 \mathrm{E}+04$ & $3.60 \mathrm{E}+04$ & $7.96 \mathrm{E}+04$ & $8.32 E+04$ & $4.94 \mathrm{E}+03$ \\
\hline 241-AX-101 & Supernatant & $3.90 \mathrm{E}+04$ & $0.00 \mathrm{E}+00$ & $0.00 \mathrm{E}+00$ & $0.00 \mathrm{E}+00$ & $0.00 \mathrm{E}+00$ & $0.00 \mathrm{E}+00$ \\
\hline 241-AX-101 & Solid & $3.90 \mathrm{E}+04$ & $3.98 \mathrm{E}+03$ & $1.73 \mathrm{E}+05$ & $2.72 \mathrm{E}+05$ & $2.82 \mathrm{E}+05$ & $5.03 E+04$ \\
\hline $241-\mathrm{AX}-102$ & Supernatant & $0.00 \mathrm{E}+00$ & $0.00 \mathrm{E}+00$ & $0.00 \mathrm{E}+00$ & $0.00 \mathrm{E}+00$ & $0.00 \mathrm{E}+00$ & $0.00 \mathrm{E}+00$ \\
\hline 241-AX-102 & Solid & $3.89 \mathrm{E}+04$ & $2.30 \mathrm{E}+04$ & $1.07 \mathrm{E}+05$ & $1.76 \mathrm{E}+05$ & $1.98 \mathrm{E}+05$ & $2.75 \mathrm{E}+04$ \\
\hline $241-\mathrm{AX}-103$ & Supernatant & $4.81 \mathrm{E}+04$ & $0.00 \mathrm{E}+00$ & $0.00 \mathrm{E}+00$ & $0.00 \mathrm{E}+00$ & $0.00 \mathrm{E}+00$ & $0.00 \mathrm{E}+00$ \\
\hline 241-AX-103 & Solid & $4.81 \mathrm{E}+04$ & $4.03 E+03$ & $1.22 \mathrm{E}+05$ & $1.91 \mathrm{E}+05$ & $2.36 \mathrm{E}+05$ & $3.79 \mathrm{E}+04$ \\
\hline 241-AX-104 & Supernatant & $0.00 \mathrm{E}+00$ & $0.00 \mathrm{E}+00$ & $0.00 \mathrm{E}+00$ & $0.00 \mathrm{E}+00$ & $0.00 \mathrm{E}+00$ & $0.00 \mathrm{E}+00$ \\
\hline 241-AX-104 & Solid & $3.89 \mathrm{E}+04$ & $2.98 \mathrm{E}+03$ & $3.60 \mathrm{E}+04$ & $7.96 \mathrm{E}+04$ & $8.32 \mathrm{E}+04$ & $4.94 \mathrm{E}+03$ \\
\hline 241-B-101 & Supernatant & $3.37 \mathrm{E}+04$ & $0.00 \mathrm{E}+00$ & $0.00 \mathrm{E}+00$ & $0.00 \mathrm{E}+00$ & $0.00 \mathrm{E}+00$ & $0.00 \mathrm{E}+00$ \\
\hline $241-\mathrm{B}-101$ & Solid & $3.37 \mathrm{E}+04$ & $6.77 \mathrm{E}+00$ & $1.32 \mathrm{E}+05$ & $3.56 \mathrm{E}+05$ & $2.26 \mathrm{E}+05$ & $7.23 \mathrm{E}+02$ \\
\hline 241-B-102 & Supernatant & $1.20 \mathrm{E}+03$ & $3.38 \mathrm{E}+00$ & $8.47 E+03$ & $2.17 \mathrm{E}+05$ & $1.13 E+05$ & $3.61 \mathrm{E}+02$ \\
\hline 241-B-102 & Solid & $1.20 \mathrm{E}+03$ & $3.38 \mathrm{E}+00$ & $8.52 \mathrm{E}+03$ & $2.17 \mathrm{E}+05$ & $1.13 E+05$ & $3.61 \mathrm{E}+02$ \\
\hline $241-\mathrm{B}-103$ & Supernatant & $1.83 \mathrm{E}+03$ & $0.00 \mathrm{E}+00$ & $0.00 \mathrm{E}+00$ & $0.00 \mathrm{E}+00$ & $0.00 \mathrm{E}+00$ & $0.00 \mathrm{E}+00$ \\
\hline 241-B-103 & Solid & $1.83 \mathrm{E}+03$ & $3.38 \mathrm{E}+00$ & $8.50 \mathrm{E}+03$ & $2.18 \mathrm{E}+05$ & $1.13 E+05$ & $3.62 \mathrm{E}+02$ \\
\hline 241-B-104 & Supernatant & $5.77 \mathrm{E}+00$ & $0.00 \mathrm{E}+00$ & $0.00 \mathrm{E}+00$ & $0.00 \mathrm{E}+00$ & $0.00 \mathrm{E}+00$ & $0.00 \mathrm{E}+00$ \\
\hline 241-B-104 & Solid & $5.77 \mathrm{E}+00$ & $3.38 \mathrm{E}+00$ & $8.49 \mathrm{E}+03$ & $2.16 \mathrm{E}+05$ & $1.13 E+05$ & $3.62 \mathrm{E}+02$ \\
\hline 241-B-105 & Supernatant & $5.16 \mathrm{E}+03$ & $0.00 \mathrm{E}+00$ & $0.00 \mathrm{E}+00$ & $0.00 \mathrm{E}+00$ & $0.00 \mathrm{E}+00$ & $0.00 \mathrm{E}+00$ \\
\hline $241-B-105$ & Solid & $5.16 \mathrm{E}+03$ & $3.38 \mathrm{E}+00$ & $8.51 \mathrm{E}+03$ & $2.16 \mathrm{E}+05$ & $1.13 E+05$ & $3.62 \mathrm{E}+02$ \\
\hline 241-B-106 & Supernatant & $5.24 \mathrm{E}+00$ & $3.38 \mathrm{E}+00$ & $8.50 \mathrm{E}+03$ & $2.17 \mathrm{E}+05$ & $1.13 \mathrm{E}+05$ & $3.60 \mathrm{E}+02$ \\
\hline 241-B-106 & Solid & $5.24 \mathrm{E}+00$ & $3.38 \mathrm{E}+00$ & $8.50 \mathrm{E}+03$ & $2.17 \mathrm{E}+05$ & $1.13 E+05$ & $3.60 \mathrm{E}+02$ \\
\hline 241-B-107 & Supernatant & $5.39 \mathrm{E}+00$ & $0.00 \mathrm{E}+00$ & $0.00 \mathrm{E}+00$ & $0.00 \mathrm{E}+00$ & $0.00 \mathrm{E}+00$ & $0.00 \mathrm{E}+00$ \\
\hline 241-B-107 & Solid & $5.39 \mathrm{E}+00$ & $5.04 \mathrm{E}+00$ & $6.51 \mathrm{E}+03$ & $3.31 E+05$ & $1.46 \mathrm{E}+05$ & $2.01 E+01$ \\
\hline 241-B-108 & Supernatant & $2.42 \mathrm{E}+04$ & $0.00 \mathrm{E}+00$ & $0.00 \mathrm{E}+00$ & $0.00 \mathrm{E}+00$ & $0.00 \mathrm{E}+00$ & $0.00 \mathrm{E}+00$ \\
\hline 241-B-108 & Solid & $2.42 E+04$ & $3.71 \mathrm{E}+00$ & $5.38 \mathrm{E}+04$ & $3.76 \mathrm{E}+05$ & $1.74 \mathrm{E}+05$ & $8.98 \mathrm{E}+02$ \\
\hline 241-B-109 & Supernatant & $1.89 \mathrm{E}+03$ & $0.00 \mathrm{E}+00$ & $0.00 \mathrm{E}+00$ & $0.00 \mathrm{E}+00$ & $0.00 \mathrm{E}+00$ & $0.00 \mathrm{E}+00$ \\
\hline 241-B-109 & Solid & $1.89 \mathrm{E}+03$ & $3.39 \mathrm{E}+00$ & $8.51 E+03$ & $2.17 \mathrm{E}+05$ & $1.13 \mathrm{E}+05$ & $3.61 \mathrm{E}+02$ \\
\hline $241-B-110$ & Supernatant & $5.56 \mathrm{E}+03$ & $5.55 \mathrm{E}+03$ & $3.95 \mathrm{E}+04$ & $7.78 \mathrm{E}+04$ & $9.55 \mathrm{E}+04$ & $1.04 \mathrm{E}+04$ \\
\hline 241-B-110 & Solid & $5.56 \mathrm{E}+03$ & $5.55 E+03$ & $3.95 \mathrm{E}+04$ & $7.78 E+04$ & $9.55 \mathrm{E}+04$ & $1.04 \mathrm{E}+04$ \\
\hline 241-B-111 & Supernatant & $4.35 \mathrm{E}-02$ & $5.55 \mathrm{E}+03$ & $3.95 \mathrm{E}+04$ & $7.78 \mathrm{E}+04$ & $9.55 \mathrm{E}+04$ & $1.04 \mathrm{E}+04$ \\
\hline 241-B-111 & Solid & $4.35 \mathrm{E}-02$ & $5.55 \mathrm{E}+03$ & $3.95 \mathrm{E}+04$ & $7.78 \mathrm{E}+04$ & $9.55 \mathrm{E}+04$ & $1.04 \mathrm{E}+04$ \\
\hline $241-\mathrm{B}-112$ & Supernatant & $1.51 \mathrm{E}+04$ & $1.82 \mathrm{E}+04$ & $1.17 \mathrm{E}+05$ & $2.17 \mathrm{E}+05$ & $2.55 \mathrm{E}+05$ & $4.73 \mathrm{E}+04$ \\
\hline 241-B-112 & Solid & $1.51 \mathrm{E}+04$ & $1.82 E+04$ & $1.17 \mathrm{E}+05$ & $2.17 \mathrm{E}+05$ & $2.55 E+05$ & $4.73 \mathrm{E}+04$ \\
\hline
\end{tabular}


Table A-1. Tank Waste Chemical Concentrations.

\begin{tabular}{|c|c|c|c|c|c|c|c|}
\hline Tank & Layer & $\underset{(\mu \mathrm{g} / \mathrm{mL})}{\mathrm{OH}^{-}}$ & $\underset{(\mu \mathrm{g} / \mathrm{mL})}{\operatorname{TOC}}$ & $\begin{array}{c}\mathrm{NO}_{2}^{-} \\
(\mu \mathrm{g} / \mathrm{mL})\end{array}$ & $\begin{array}{c}\mathrm{NO}_{3}^{-} \\
(\mu \mathrm{g} / \mathrm{mL})\end{array}$ & $\begin{array}{c}\mathrm{Na}^{+} \\
(\mu \mathrm{g} / \mathrm{mL})\end{array}$ & $\begin{array}{c}\mathrm{Al}^{+3} \\
(\mu \mathrm{g} / \mathrm{mL})\end{array}$ \\
\hline $241-\mathrm{B}-201$ & Supernatant & $0.00 \mathrm{E}+00$ & $0.00 \mathrm{E}+00$ & $0.00 \mathrm{E}+00$ & $0.00 \mathrm{E}+00$ & $0.00 \mathrm{E}+00$ & $0.00 \mathrm{E}+00$ \\
\hline $241-\mathrm{B}-201$ & Solid & $1.70 \mathrm{E}+04$ & $2.98 \mathrm{E}+03$ & $3.60 \mathrm{E}+04$ & $7.96 \mathrm{E}+04$ & $8.32 \mathrm{E}+04$ & $4.94 \mathrm{E}+03$ \\
\hline $241-\mathrm{B}-202$ & Supernatant & $0.00 \mathrm{E}+00$ & $0.00 \mathrm{E}+00$ & $0.00 \mathrm{E}+00$ & $0.00 \mathrm{E}+00$ & $0.00 \mathrm{E}+00$ & $0.00 \mathrm{E}+00$ \\
\hline $241-\mathrm{B}-202$ & Solid & $1.70 \mathrm{E}+04$ & $2.98 \mathrm{E}+03$ & $3.60 \mathrm{E}+04$ & $7.96 \mathrm{E}+04$ & $8.32 \mathrm{E}+04$ & $4.94 \mathrm{E}+03$ \\
\hline 241-B-203 & Supernatant & $1.36 \mathrm{E}+02$ & $9.60 \mathrm{E}+01$ & $1.57 \mathrm{E}+03$ & $5.65 E+04$ & $3.12 \mathrm{E}+04$ & $0.00 \mathrm{E}+00$ \\
\hline 241-B-203 & Solid & $1.36 \mathrm{E}+02$ & $9.60 \mathrm{E}+01$ & $1.57 \mathrm{E}+03$ & $5.65 \mathrm{E}+04$ & $3.12 \mathrm{E}+04$ & $0.00 \mathrm{E}+00$ \\
\hline $241-B-204$ & Supernatant & $9.18 \mathrm{E}+02$ & $7.53 \mathrm{E}+01$ & $1.36 \mathrm{E}+03$ & $3.47 \mathrm{E}+04$ & $2.78 \mathrm{E}+04$ & $0.00 \mathrm{E}+00$ \\
\hline 241-B-204 & Solid & $9.18 \mathrm{E}+02$ & $7.53 \mathrm{E}+01$ & $1.36 \mathrm{E}+03$ & $3.47 \mathrm{E}+04$ & $2.78 \mathrm{E}+04$ & $0.00 \mathrm{E}+00$ \\
\hline 241-BX-101 & Supernatant & $0.00 \mathrm{E}+00$ & $0.00 \mathrm{E}+00$ & $0.00 \mathrm{E}+00$ & $0.00 \mathrm{E}+00$ & $0.00 \mathrm{E}+00$ & $0.00 \mathrm{E}+00$ \\
\hline 241-BX-101 & Solid & $1.70 \mathrm{E}+04$ & $4.98 \mathrm{E}+03$ & $6.43 \mathrm{E}+04$ & $1.21 \mathrm{E}+05$ & $1.42 \mathrm{E}+05$ & $5.17 \mathrm{E}+03$ \\
\hline $241-\mathrm{BX}-102$ & Supernatant & $0.00 \mathrm{E}+00$ & $0.00 \mathrm{E}+00$ & $0.00 \mathrm{E}+00$ & $0.00 \mathrm{E}+00$ & $0.00 \mathrm{E}+00$ & $0.00 \mathrm{E}+00$ \\
\hline $241-\mathrm{BX}-102$ & Solid & $1.70 \mathrm{E}+04$ & $2.98 \mathrm{E}+03$ & $3.60 \mathrm{E}+04$ & $7.96 \mathrm{E}+04$ & $8.32 \mathrm{E}+04$ & $4.94 \mathrm{E}+03$ \\
\hline $241-\mathrm{BX}-103$ & Supernatant & $5.96 \mathrm{E}+01$ & $3.64 \mathrm{E}+03$ & $1.37 \mathrm{E}+04$ & $4.44 \mathrm{E}+04$ & $3.88 \mathrm{E}+04$ & $1.77 \mathrm{E}+04$ \\
\hline $241-\mathrm{BX}-103$ & Solid & $5.96 \mathrm{E}+01$ & $3.64 \mathrm{E}+03$ & $1.37 \mathrm{E}+04$ & $4.44 \mathrm{E}+04$ & $3.88 \mathrm{E}+04$ & $1.77 \mathrm{E}+04$ \\
\hline 241-BX-104 & Superna & $2.24 \mathrm{E}+03$ & $3.19 \mathrm{E}+03$ & $6.37 \mathrm{E}+04$ & $1.34 \mathrm{E}+05$ & $1.40 \mathrm{E}+05$ & $4 \mathrm{E}+03$ \\
\hline $\mathrm{X}-104$ & Sol & $2.24 \mathrm{E}+03$ & $3.19 \mathrm{E}+03$ & $6.37 \mathrm{E}+04$ & $1.34 \mathrm{E}+05$ & $1.40 \mathrm{E}+05$ & $2.84 \mathrm{E}+03$ \\
\hline 241-BX & Supernatant & $3.77 \mathrm{E}+03$ & $9.44 \mathrm{E}+03$ & $4.00 \mathrm{E}+04$ & $1.17 \mathrm{E}+05$ & $1.24 \mathrm{E}+05$ & $4 \mathrm{E}+03$ \\
\hline 241-BX-105 & Solid & $3.77 \mathrm{E}+03$ & $9.44 \mathrm{E}+03$ & $4.00 \mathrm{E}+04$ & $1.17 \mathrm{E}+05$ & $1.24 \mathrm{E}+05$ & $5.94 \mathrm{E}+03$ \\
\hline 241-BX-106 & Supernatant & $8.90 \mathrm{E}+01$ & $0.00 \mathrm{E}+00$ & $0.00 \mathrm{E}+00$ & $0.00 \mathrm{E}+00$ & $0.00 \mathrm{E}+00$ & $0.00 \mathrm{E}+00$ \\
\hline 241-BX-106 & Solid & $8.90 \mathrm{E}+01$ & $1.82 \mathrm{E}+04$ & $1.17 \mathrm{E}+05$ & $2.17 \mathrm{E}+05$ & $2.55 \mathrm{E}+05$ & $4.72 \mathrm{E}+04$ \\
\hline 241-BX-107 & Supernatant & $0.00 \mathrm{E}+00$ & $0.00 \mathrm{E}+00$ & $0.00 \mathrm{E}+00$ & $0.00 \mathrm{E}+00$ & $0.00 \mathrm{E}+00$ & $0.00 \mathrm{E}+00$ \\
\hline 241-BX-107 & Solid & $1.70 \mathrm{E}+04$ & $2.98 \mathrm{E}+03$ & $3.60 \mathrm{E}+04$ & $7.96 \mathrm{E}+04$ & $8.32 \mathrm{E}+04$ & $4.94 \mathrm{E}+03$ \\
\hline 241-BX-108 & Supernatant & $0.00 \mathrm{E}+00$ & $0.00 \mathrm{E}+00$ & $0.00 \mathrm{E}+00$ & $0.00 \mathrm{E}+00$ & $0.00 \mathrm{E}+00$ & $0.00 \mathrm{E}+00$ \\
\hline $241-\mathrm{BX}-108$ & & $1.70 \mathrm{E}+04$ & $2.98 \mathrm{E}+03$ & $3.60 \mathrm{E}+04$ & $7.96 \mathrm{E}+04$ & $8.32 \mathrm{E}+04$ & $\mathrm{E}+03$ \\
\hline $241-B$ & Super & $0.00 \mathrm{E}+00$ & $0.00 \mathrm{E}+00$ & $0.00 \mathrm{E}+00$ & $0.00 \mathrm{E}+00$ & $0.00 \mathrm{E}+00$ & $0 \mathrm{E}+00$ \\
\hline 241-BX-109 & Sol & $1.70 \mathrm{E}+04$ & $2.98 \mathrm{E}+03$ & $3.60 \mathrm{E}+04$ & $7.96 \mathrm{E}+04$ & $8.32 \mathrm{E}+04$ & $4.94 \mathrm{E}+03$ \\
\hline-110 & upernatant & $2.35 \mathrm{E}+04$ & $1.52 \mathrm{E}+04$ & $5.22 \mathrm{E}+04$ & $4.12 \mathrm{E}+05$ & $2.24 \mathrm{E}+05$ & $4.40 \mathrm{E}+03$ \\
\hline $241-\mathrm{BX}-110$ & Solid & $2.35 E+04$ & $1.52 \mathrm{E}+04$ & $5.21 \mathrm{E}+04$ & $4.12 \mathrm{E}+05$ & $2.23 \mathrm{E}+05$ & $4.40 \mathrm{E}+03$ \\
\hline 241-BX-111 & Supernatant & $6.93 \mathrm{E}+04$ & $0.00 \mathrm{E}+00$ & $0.00 \mathrm{E}+00$ & $0.00 \mathrm{E}+00$ & $0.00 \mathrm{E}+00$ & $0.00 \mathrm{E}+00$ \\
\hline $\mathrm{X}-111$ & & $6.93 \mathrm{E}+04$ & $1.70 \mathrm{E}+03$ & $1.06 \mathrm{E}+05$ & $1.85 \mathrm{E}+05$ & $2.26 \mathrm{E}+05$ & $4.87 \mathrm{E}+04$ \\
\hline 241-BX-112 & Supernatant & $2.70 \mathrm{E}+03$ & $0.00 \mathrm{E}+00$ & $3.52 \mathrm{E}+04$ & $9.86 \mathrm{E}+04$ & $7.78 \mathrm{E}+04$ & $4.78 \mathrm{E}+01$ \\
\hline 241-BX-112 & Solid & $2.70 \mathrm{E}+03$ & $0.00 \mathrm{E}+00$ & $3.52 \mathrm{E}+04$ & $9.86 \mathrm{E}+04$ & $7.78 \mathrm{E}+04$ & $4.78 E+01$ \\
\hline 241-BY-101 & Supernatant & $7.00 \mathrm{E}+04$ & $0.00 \mathrm{E}+00$ & $0.00 \mathrm{E}+00$ & $0.00 \mathrm{E}+00$ & $0.00 \mathrm{E}+00$ & $0.00 \mathrm{E}+00$ \\
\hline 241-BY-101 & & $7.00 \mathrm{E}+04$ & $1.82 \mathrm{E}+04$ & $1.17 \mathrm{E}+05$ & $2.17 \mathrm{E}+05$ & $2.56 \mathrm{E}+05$ & $4.73 \mathrm{E}+04$ \\
\hline-102 & $\tan t$ & $\overline{E+04}$ & 0.00 & $E+00$ & $2+00$ & $0.00 \mathrm{E}+00$ & $\overline{E+00}$ \\
\hline 241-BY-102 & & $5.62 \mathrm{E}+04$ & $1.55 \mathrm{E}+03$ & $8.41 \mathrm{E}+04$ & $1.48 \mathrm{E}+05$ & $2.34 \mathrm{E}+05$ & $5.66 \mathrm{E}+04$ \\
\hline 241-BY-103 & upernatant & $3.15 \mathrm{E}+04$ & $0.00 \mathrm{E}+00$ & $0.00 \mathrm{E}+00$ & $0.00 \mathrm{E}+00$ & $0.00 \mathrm{E}+00$ & $0.00 \mathrm{E}+00$ \\
\hline 241-BY-103 & Solid & $3.15 \mathrm{E}+04$ & $1.59 \mathrm{E}+03$ & $4.49 \mathrm{E}+04$ & $1.41 \mathrm{E}+05$ & $1.80 \mathrm{E}+05$ & $3.46 \mathrm{E}+04$ \\
\hline 241-BY-104 & upernatant & $1.68 \mathrm{E}+04$ & $0.00 \mathrm{E}+00$ & $0.00 \mathrm{E}+00$ & $0.00 \mathrm{E}+00$ & $0.00 \mathrm{E}+00$ & $0.00 \mathrm{E}+00$ \\
\hline 241-BY-104 & Solid & $1.68 \mathrm{E}+04$ & $2.37 \mathrm{E}+03$ & $1.11 \mathrm{E}+05$ & $2.07 \mathrm{E}+05$ & $2.48 \mathrm{E}+05$ & $7.19 \mathrm{E}+04$ \\
\hline 241-BY-105 & Supernatant & $3.83 \mathrm{E}+04$ & $0.00 \mathrm{E}+00$ & $0.00 \mathrm{E}+00$ & $0.00 \mathrm{E}+00$ & $0.00 \mathrm{E}+00$ & $0.00 \mathrm{E}+00$ \\
\hline 241-BY-105 & Solid & $3.83 \mathrm{E}+04$ & $2.43 \mathrm{E}+03$ & $6.86 \mathrm{E}+04$ & $1.99 \mathrm{E}+05$ & $2.08 \mathrm{E}+05$ & $3.92 \mathrm{E}+04$ \\
\hline 241-BY-106 & Supernatant & $3.58 \mathrm{E}+04$ & $0.00 \mathrm{E}+00$ & $0.00 \mathrm{E}+00$ & $0.00 \mathrm{E}+00$ & $0.00 \mathrm{E}+00$ & $0.00 \mathrm{E}+00$ \\
\hline 241-BY-106 & Solid & $3.58 \mathrm{E}+04$ & $2.67 \mathrm{E}+03$ & $7.83 \mathrm{E}+04$ & $1.10 \mathrm{E}+05$ & $1.64 \mathrm{E}+05$ & $3.98 \mathrm{E}+04$ \\
\hline 241-BY-107 & Supernatant & $7.07 \mathrm{E}+04$ & $0.00 \mathrm{E}+00$ & $0.00 \mathrm{E}+00$ & $0.00 \mathrm{E}+00$ & $0.00 \mathrm{E}+00$ & $0.00 \mathrm{E}+00$ \\
\hline 241-BY-107 & Solid & $7.07 \mathrm{E}+04$ & $2.40 \mathrm{E}+03$ & $1.17 \mathrm{E}+05$ & $1.31 \mathrm{E}+05$ & $2.43 \mathrm{E}+05$ & $5.12 E+04$ \\
\hline 241-BY-108 & Supernatant & $8.38 \mathrm{E}+04$ & $0.00 \mathrm{E}+00$ & $0.00 \mathrm{E}+00$ & $0.00 \mathrm{E}+00$ & $0.00 \mathrm{E}+00$ & $0.00 \mathrm{E}+00$ \\
\hline 241-BY-108 & & $8.38 \mathrm{E}+04$ & $2.37 \mathrm{E}+03$ & $7.85 \mathrm{E}+04$ & $2.94 \mathrm{E}+05$ & $2.82 \mathrm{E}+05$ & $3.63 \mathrm{E}+04$ \\
\hline 241-BY-109 & Supernatant & $3.93 \mathrm{E}+04$ & $0.00 \mathrm{E}+00$ & $0.00 \mathrm{E}+00$ & $0.00 \mathrm{E}+00$ & $0.00 \mathrm{E}+00$ & $0.00 \mathrm{E}+00$ \\
\hline 241-BY-109 & Solid & $3.93 \mathrm{E}+04$ & $1.88 \mathrm{E}+03$ & $8.29 E+04$ & $1.90 \mathrm{E}+05$ & $2.10 \mathrm{E}+05$ & $3.88 \mathrm{E}+04$ \\
\hline
\end{tabular}


Table A-1. Tank Waste Chemical Concentrations.

\begin{tabular}{|c|c|c|c|c|c|c|c|}
\hline Tank & Layer & $\underset{(\mu \mathrm{g} / \mathbf{m L})}{\mathrm{OH}^{-}}$ & $\begin{array}{c}\text { TOC } \\
(\mu \mathrm{g} / \mathrm{mL})\end{array}$ & $\begin{array}{c}\mathrm{NO}_{2}^{-} \\
(\mu \mathrm{g} / \mathrm{mL})\end{array}$ & $\begin{array}{c}\mathrm{NO}_{3}^{-} \\
(\mu \mathrm{g} / \mathrm{mL})\end{array}$ & $\begin{array}{c}\mathrm{Na}^{+} \\
(\mu \mathrm{g} / \mathrm{mL})\end{array}$ & $\begin{array}{c}\mathrm{Al}^{+3} \\
(\mu \mathrm{g} / \mathrm{mL})\end{array}$ \\
\hline 241-BY-110 & Supernatant & $5.11 \mathrm{E}+04$ & $0.00 \mathrm{E}+00$ & $0.00 \mathrm{E}+00$ & $0.00 \mathrm{E}+00$ & $0.00 \mathrm{E}+00$ & $0.00 \mathrm{E}+00$ \\
\hline $241-\mathrm{BY}-110$ & Solid & $5.11 \mathrm{E}+04$ & $1.94 \mathrm{E}+04$ & $1.24 \mathrm{E}+05$ & $1.24 \mathrm{E}+05$ & $2.54 \mathrm{E}+05$ & $6.58 \mathrm{E}+04$ \\
\hline 241-BY-111 & Supernatant & $6.05 \mathrm{E}+04$ & $0.00 \mathrm{E}+00$ & $0.00 \mathrm{E}+00$ & $0.00 \mathrm{E}+00$ & $0.00 \mathrm{E}+00$ & $0.00 \mathrm{E}+00$ \\
\hline 241-BY-111 & Solid & $6.05 \mathrm{E}+04$ & $1.28 \mathrm{E}+03$ & $5.46 \mathrm{E}+04$ & $2.01 \mathrm{E}+05$ & $2.17 \mathrm{E}+05$ & $4.30 \mathrm{E}+04$ \\
\hline 241-BY-112 & Supernatant & $8.79 E+04$ & $0.00 \mathrm{E}+00$ & $0.00 \mathrm{E}+00$ & $0.00 \mathrm{E}+00$ & $0.00 \mathrm{E}+00$ & $0.00 \mathrm{E}+00$ \\
\hline $241-\mathrm{BY}-112$ & Solid & $8.79 \mathrm{E}+04$ & $1.72 \mathrm{E}+03$ & $1.41 \mathrm{E}+05$ & $1.90 \mathrm{E}+05$ & $2.44 \mathrm{E}+05$ & $5.54 \mathrm{E}+04$ \\
\hline 241-C-101 & Supernatant & $0.00 \mathrm{E}+00$ & $0.00 \mathrm{E}+00$ & $0.00 \mathrm{E}+00$ & $0.00 \mathrm{E}+00$ & $0.00 \mathrm{E}+00$ & $0.00 \mathrm{E}+00$ \\
\hline $241-C-101$ & Solid & $1.70 \mathrm{E}+04$ & $2.98 \mathrm{E}+03$ & $3.60 \mathrm{E}+04$ & $7.96 \mathrm{E}+04$ & $8.32 \mathrm{E}+04$ & $4.94 \mathrm{E}+03$ \\
\hline $241-\mathrm{C}-102$ & upernatant & $0.00 \mathrm{E}+00$ & $0.00 \mathrm{E}+00$ & $0.00 \mathrm{E}+00$ & $0.00 \mathrm{E}+00$ & $0.00 \mathrm{E}+00$ & $0.00 \mathrm{E}+00$ \\
\hline 241-C-102 & Solid & $1.70 \mathrm{E}+04$ & $2.98 \mathrm{E}+03$ & $3.60 \mathrm{E}+04$ & $7.96 \mathrm{E}+04$ & $8.32 E+04$ & $4.94 \mathrm{E}+03$ \\
\hline $241-C-103$ & Supernatant & $1.01 \mathrm{E}-01$ & $5.30 \mathrm{E}-01$ & $3.85 \mathrm{E}+01$ & $5.13 \mathrm{E}+01$ & $1.26 \mathrm{E}+02$ & $1.20 \mathrm{E}+00$ \\
\hline $241-\mathrm{C}-103$ & Solid & $1.01 \mathrm{E}-01$ & $5.30 \mathrm{E}-01$ & $3.85 \mathrm{E}+01$ & $5.13 \mathrm{E}+01$ & $1.26 \mathrm{E}+02$ & $1.20 \mathrm{E}+00$ \\
\hline $241-\mathrm{C}-104$ & Supernatant & $0.00 \mathrm{E}+00$ & $0.00 \mathrm{E}+00$ & $0.00 \mathrm{E}+00$ & $0.00 \mathrm{E}+00$ & $0.00 \mathrm{E}+00$ & $0.00 \mathrm{E}+00$ \\
\hline $241-\mathrm{C}-104$ & Solid & $1.70 \mathrm{E}+04$ & $5.60 \mathrm{E}+03$ & $3.60 \mathrm{E}+04$ & $7.96 \mathrm{E}+04$ & $8.32 \mathrm{E}+04$ & $4.94 \mathrm{E}+03$ \\
\hline $241-\mathrm{C}-105$ & Supernatant & $0.00 \mathrm{E}+00$ & $0.00 \mathrm{E}+00$ & $0.00 \mathrm{E}+00$ & $0.00 \mathrm{E}+00$ & $0.00 \mathrm{E}+00$ & $0.00 \mathrm{E}+00$ \\
\hline 241-C-105 & & $1.70 \mathrm{E}+04$ & $2.87 \mathrm{E}+03$ & $3.70 \mathrm{E}+04$ & $3.43 E+04$ & $1.14 \mathrm{E}+05$ & $9.02 E+00$ \\
\hline & $\tan t$ & $7.02 \mathrm{E}$ & & $5.43 \mathrm{E}+01$ & & $9.78 \mathrm{E}+03$ & $9.38 \mathrm{E}+01$ \\
\hline & & 7.02 & & $5.43 \mathrm{E}+01$ & 6.02 & $9.78 \mathrm{E}+03$ & $\mathrm{E}+01$ \\
\hline 241 & $\operatorname{ant}$ & 0.00 & +00 & $0.00 \mathrm{E}+00$ & 0.00 & $0.00 \mathrm{E}+00$ & $\mathrm{E}+00$ \\
\hline & & 1.70 & +03 & $3.60 \mathrm{E}+04$ & 7.96 & $8.32 \mathrm{E}+04$ & $\mathrm{E}+03$ \\
\hline & $\operatorname{tant}$ & 6.83 & +02 & $2.86 \mathrm{E}+04$ & $E+03$ & $5.37 \mathrm{E}+04$ & $5 \mathrm{E}+03$ \\
\hline & & & & $2.86 \mathrm{E}+04$ & & $5.37 \mathrm{E}+04$ & $E+03$ \\
\hline $241-C-109$ & upernatant & $0.00 \mathrm{E}$ & & $0.00 \mathrm{E}+00$ & $0.00 \mathrm{E}+00$ & $0.00 \mathrm{E}+00$ & $0.00 \mathrm{E}+00$ \\
\hline $241-C$ & Solid & $1.70 \mathrm{E}+04$ & $2.98 \mathrm{E}+03$ & $3.60 \mathrm{E}+04$ & $7.96 \mathrm{E}+04$ & $8.32 \mathrm{E}+04$ & $4.94 \mathrm{E}+03$ \\
\hline $241-\mathrm{C}$ & natant & $1.70 \mathrm{E}+04$ & $0.00 \mathrm{E}+00$ & $0.00 \mathrm{E}+00$ & $0.00 \mathrm{E}+00$ & $0.00 \mathrm{E}+00$ & $0.00 \mathrm{E}+00$ \\
\hline $241-\mathrm{C}-110$ & & $1.70 \mathrm{E}+04$ & $5.80 \mathrm{E}+02$ & $4.60 \mathrm{E}+03$ & $6.03 E+04$ & $3.05 \mathrm{E}+04$ & $2.09 \mathrm{E}+02$ \\
\hline & Supe & & $0.00 \mathrm{E}+00$ & $0.00 \mathrm{E}+00$ & $0.00 \mathrm{E}+00$ & $0.00 \mathrm{E}+00$ & $0.00 \mathrm{E}+00$ \\
\hline & & & & & & $8.32 \mathrm{E}+04$ & $4 \mathrm{E}+03$ \\
\hline & & & & 00 & 0.00 & $0.00 \mathrm{E}+00$ & $E+00$ \\
\hline 241 & & 1.70 & +03 & 3.60 & 7.96 & $8.32 \mathrm{E}+04$ & $\mathrm{E}+03$ \\
\hline & Supe & 1.70 & +00 & 0.00 & 0.00 & $0.00 \mathrm{E}+00$ & $E+00$ \\
\hline & & 1.70 & +00 & $0.00 \mathrm{E}+00$ & +00 & $0.00 \mathrm{E}+00$ & $\mathrm{E}+00$ \\
\hline 241 & tent & +04 & +00 & +00 & +00 & $0.00 \mathrm{E}+00$ & $0 \mathrm{E}+00$ \\
\hline $241-C$ & & $1.70 \mathrm{E}+04$ & $\mathrm{E}+00$ & $0.00 \mathrm{E}+00$ & $0.00 \mathrm{E}+00$ & $0.00 \mathrm{E}+00$ & $0.00 \mathrm{E}+00$ \\
\hline $241-\mathrm{C}$ & Supernatant & $1.70 \mathrm{E}+04$ & $0.00 \mathrm{E}+00$ & $0.00 \mathrm{E}+00$ & $0.00 \mathrm{E}+00$ & $0.00 \mathrm{E}+00$ & $0.00 \mathrm{E}+00$ \\
\hline $241-\mathrm{C}-203$ & Solid & $1.70 \mathrm{E}+04$ & $0.00 \mathrm{E}+00$ & $0.00 \mathrm{E}+00$ & $0.00 \mathrm{E}+00$ & $0.00 \mathrm{E}+00$ & $0.00 \mathrm{E}+00$ \\
\hline $241-C-204$ & Supernatant & $1.70 \mathrm{E}+04$ & $0.00 \mathrm{E}+00$ & $0.00 \mathrm{E}+00$ & $0.00 \mathrm{E}+00$ & $0.00 \mathrm{E}+00$ & $0.00 \mathrm{E}+00$ \\
\hline 241-C-204 & Solid & $1.70 \mathrm{E}+04$ & $0.00 \mathrm{E}+00$ & $0.00 \mathrm{E}+00$ & $0.00 \mathrm{E}+00$ & $0.00 \mathrm{E}+00$ & $0.00 \mathrm{E}+00$ \\
\hline & Supernatant & $4.76 \mathrm{E}+04$ & $0.00 \mathrm{E}+00$ & $0.00 \mathrm{E}+00$ & $0.00 \mathrm{E}+00$ & $0.00 \mathrm{E}+00$ & $0.00 \mathrm{E}+00$ \\
\hline $241-S-101$ & & & $1.79 \mathrm{E}+03$ & $1.13 \mathrm{E}+05$ & $2.14 \mathrm{E}+05$ & $2.22 \mathrm{E}+05$ & $2.99 \mathrm{E}+04$ \\
\hline & & & & $0.00 \mathrm{E}+00$ & $0.00 \mathrm{E}+00$ & $0.00 \mathrm{E}+00$ & $0 \mathrm{E}+00$ \\
\hline & & 3.76 & $3.76 \mathrm{E}+03$ & $1.27 \mathrm{E}+05$ & $1.55 \mathrm{E}+05$ & $2.34 \mathrm{E}+05$ & $5.62 \mathrm{E}+04$ \\
\hline & $\tan >>$ & & +03 & $1.47 \mathrm{E}+05$ & 2.16 & $2.28 \mathrm{E}+05$ & $3.20 \mathrm{E}+04$ \\
\hline & & $4.11 \mathrm{E}+02$ & $4.68 \mathrm{E}+03$ & $1.47 \mathrm{E}+05$ & $2.16 \mathrm{E}+05$ & $2.28 \mathrm{E}+05$ & $3.20 \mathrm{E}+04$ \\
\hline $241-S$ & $\operatorname{tant}$ & $5.10 \mathrm{E}+03$ & $0.00 \mathrm{E}+00$ & $0.00 \mathrm{E}+00$ & $0.00 \mathrm{E}+00$ & $0.00 \mathrm{E}+00$ & $0.00 \mathrm{E}+00$ \\
\hline $241-5$ & & +03 & $3.96 \mathrm{E}+02$ & $3.82 \mathrm{E}+04$ & $2.68 \mathrm{E}+05$ & $1.98 \mathrm{E}+05$ & $9 \mathrm{E}+04$ \\
\hline $241-S$ & upernatant & $4.81 \mathrm{E}+04$ & $0.00 \mathrm{E}+00$ & $0.00 \mathrm{E}+00$ & $0.00 \mathrm{E}+00$ & $0.00 \mathrm{E}+00$ & $0.00 \mathrm{E}+00$ \\
\hline $241-S-105$ & Solid & $4.81 \mathrm{E}+04$ & $4.03 \mathrm{E}+03$ & $1.22 \mathrm{E}+05$ & $1.90 \mathrm{E}+05$ & $2.36 \mathrm{E}+05$ & $3.78 \mathrm{E}+04$ \\
\hline 241-S-106 & Supernatant & $5.55 \mathrm{E}+04$ & $0.00 \mathrm{E}+00$ & $0.00 \mathrm{E}+00$ & $0.00 \mathrm{E}+00$ & $0.00 \mathrm{E}+00$ & $0.00 \mathrm{E}+00$ \\
\hline $241-S-106$ & Solid & $5.55 \mathrm{E}+04$ & $1.98 \mathrm{E}+03$ & $1.04 \mathrm{E}+05$ & $2.36 \mathrm{E}+05$ & $2.44 \mathrm{E}+05$ & $3.80 \mathrm{E}+04$ \\
\hline
\end{tabular}


Table A-1. Tank Waste Chemical Concentrations.

\begin{tabular}{|c|c|c|c|c|c|c|c|}
\hline Tank & Layer & $\begin{array}{c}\mathbf{O H} \\
(\mu \mathrm{g} / \mathrm{mL})\end{array}$ & $\begin{array}{c}\text { TOC } \\
(\mu \mathrm{g} / \mathrm{mL})\end{array}$ & $\begin{array}{c}\mathrm{NO}_{2}^{-} \\
(\mu \mathrm{g} / \mathrm{mL})\end{array}$ & $\begin{array}{c}\mathrm{NO}_{3}^{-} \\
(\mu \mathrm{g} / \mathrm{mL})\end{array}$ & $\begin{array}{c}\mathrm{Na}^{+} \\
(\mu \mathrm{g} / \mathrm{mL}) \\
\end{array}$ & $\begin{array}{c}\mathbf{A l}^{+3} \\
(\mu \mathrm{g} / \mathrm{mL})\end{array}$ \\
\hline $241-S-107$ & Supernatant & $0.00 \mathrm{E}+00$ & $0.00 \mathrm{E}+00$ & $0.00 \mathrm{E}+00$ & $0.00 \mathrm{E}+00$ & $0.00 \mathrm{E}+00$ & $0.00 \mathrm{E}+00$ \\
\hline $241-S-107$ & Solid & $2.99 \mathrm{E}+04$ & $1.18 E+03$ & $7.11 \mathrm{E}+04$ & $1.14 \mathrm{E}+05$ & $1.56 \mathrm{E}+05$ & $1.07 \mathrm{E}+04$ \\
\hline 241-S-108 & upernatant & $4.81 \mathrm{E}+04$ & $0.00 \mathrm{E}+00$ & $0.00 \mathrm{E}+00$ & $0.00 \mathrm{E}+00$ & $0.00 \mathrm{E}+00$ & $0.00 \mathrm{E}+00$ \\
\hline $241-S-108$ & Solid & $.81 \mathrm{E}+04$ & $4.03 E+03$ & $1.22 \mathrm{E}+05$ & $1.90 \mathrm{E}+05$ & $2.36 \mathrm{E}+05$ & $3.77 \mathrm{E}+04$ \\
\hline 241-S-109 & upernatant & $8.19 \mathrm{E}+04$ & $0.00 \mathrm{E}+00$ & $0.00 \mathrm{E}+00$ & $0.00 \mathrm{E}+00$ & $0.00 \mathrm{E}+00$ & $0.00 \mathrm{E}+00$ \\
\hline 241-S-109 & Solid & $8.19 \mathrm{E}+04$ & $1.11 \mathrm{E}+03$ & $8.49 E+04$ & $1.32 \mathrm{E}+05$ & $2.19 \mathrm{E}+05$ & $4.59 \mathrm{E}+04$ \\
\hline $241-S-110$ & Supernatant & $4.72 \mathrm{E}+04$ & $0.00 \mathrm{E}+00$ & $0.00 \mathrm{E}+00$ & $0.00 \mathrm{E}+00$ & $0.00 \mathrm{E}+00$ & $0.00 \mathrm{E}+00$ \\
\hline $241-\mathrm{S}-110$ & Solid & $4.72 E+04$ & $2.28 \mathrm{E}+03$ & $8.58 \mathrm{E}+04$ & $2.17 \mathrm{E}+05$ & $2.11 \mathrm{E}+05$ & $3.10 \mathrm{E}+04$ \\
\hline $241-S-111$ & Supernatant & $5.10 \mathrm{E}+04$ & $0.00 \mathrm{E}+00$ & $0.00 \mathrm{E}+00$ & $0.00 \mathrm{E}+00$ & $0.00 \mathrm{E}+00$ & $0.00 \mathrm{E}+00$ \\
\hline $241-\mathrm{S}-111$ & Solid & $5.10 \mathrm{E}+04$ & $1.49 \mathrm{E}+03$ & $8.39 \mathrm{E}+04$ & $1.93 \mathrm{E}+05$ & $2.23 E+05$ & $3.64 \mathrm{E}+04$ \\
\hline $241-S-112$ & uper & & $0.00 \mathrm{E}+00$ & & $0.00 \mathrm{E}+00$ & $1.56 \mathrm{E}+05$ & $1.88 \mathrm{E}+04$ \\
\hline $241-$ & & $E+04$ & 0.00 & 0.00 & $0.00 \mathrm{E}+00$ & $1.56 \mathrm{E}+05$ & $\mathrm{E}+04$ \\
\hline 241 & $\operatorname{tant}$ & +04 & 0.00 & 0.00 & $0.00 \mathrm{E}+00$ & $\mathrm{E}+00$ & $\mathrm{E}+00$ \\
\hline $241-\mathrm{S}$ & & +04 & 3.35 & 7.49 & $1.08 \mathrm{E}+05$ & $E+05$ & $\mathrm{E}+04$ \\
\hline 241 & Super & +04 & 0.00 & 0.00 & $0.00 \mathrm{E}+00$ & $8+00$ & $\mathrm{E}+00$ \\
\hline $241-\mathrm{SX}-102$ & & $5.01 \mathrm{E}+04$ & 1.51 & 1.51 & $1.57 \mathrm{E}+05$ & $\overline{E+05}$ & $E+04$ \\
\hline $241-\mathrm{SX}-103$ & Supernatant & $4.52 \mathrm{E}+04$ & $0.00 \mathrm{E}+00$ & $0.00 \mathrm{E}+00$ & $0.00 \mathrm{E}+00$ & $0.00 \mathrm{E}+00$ & $0.00 \mathrm{E}+00$ \\
\hline $241-S X-103$ & Solid & $4.52 \mathrm{E}+04$ & $3.38 \mathrm{E}+03$ & $1.57 \mathrm{E}+05$ & $1.56 \mathrm{E}+05$ & $2.40 \mathrm{E}+05$ & $4.44 \mathrm{E}+04$ \\
\hline 241-SX-104 & Supernatant & $3.05 \mathrm{E}+04$ & $0.00 \mathrm{E}+00$ & $0.00 \mathrm{E}+00$ & $0.00 \mathrm{E}+00$ & $0.00 \mathrm{E}+00$ & $0.00 \mathrm{E}+00$ \\
\hline$\overline{241}$ & & & & & $2.90 \mathrm{E}+05$ & $2.50 \mathrm{E}+05$ & $\mathrm{E}+04$ \\
\hline 241 & uper & & & & +00 & +00 & +00 \\
\hline & & -04 & 3.2 & 1.43 & +05 & \pm 05 & +04 \\
\hline 241 & uper & +04 & 0.00 & 0.00 & $0.00 \mathrm{E}+00$ & $\mathrm{E}+00$ & $E+00$ \\
\hline 241 & & +04 & 3.1 & 1.49 & $2.45 \mathrm{E}+05$ & $\mathrm{E}+05$ & $\mathrm{E}+04$ \\
\hline & ( & & & & 100 & +00 & $E+00$ \\
\hline 241-SX-107 & Sol & +04 & 1.01 & $9.86 \mathrm{E}+04$ & $2.17 \mathrm{E}+05$ & $2.55 \mathrm{E}+05$ & $\bar{E}+04$ \\
\hline 241-SX-108 & upernatant & $0.00 \mathrm{E}+00$ & $0.00 \mathrm{E}+00$ & $0.00 \mathrm{E}+00$ & $0.00 \mathrm{E}+00$ & $0.00 \mathrm{E}+00$ & $E+00$ \\
\hline 241-SX-108 & & $6.07 \mathrm{E}+04$ & $2.98 \mathrm{E}+03$ & $3.60 \mathrm{E}+04$ & $7.96 \mathrm{E}+04$ & $8.32 \mathrm{E}+04$ & $E+03$ \\
\hline & Unernatant & & & & & $0.00 \mathrm{E}+00$ & $\mathrm{E}+00$ \\
\hline & & & & & +05 & $8+05$ & +04 \\
\hline & & & & & +00 & 400 & \\
\hline & & +04 & 2.9 & 3.6 & +04 & +04 & +03 \\
\hline 241 & up & +00 & 0.00 & 0.00 & $0.00 \mathrm{E}+00$ & $0.00 \mathrm{E}+00$ & $E+00$ \\
\hline & & +04 & & +04 & $\mathrm{E}+05$ & $2.55 \mathrm{E}+05$ & $\mathrm{E}+04$ \\
\hline-112 & Supernatant & +00 & $0.00 \mathrm{E}+00$ & $0.00 \mathrm{E}+00$ & $0.00 \mathrm{E}+00$ & $0.00 \mathrm{E}+00$ & $\mathrm{E}+00$ \\
\hline $241-\mathrm{SX}-112$ & Solid & $6.07 \mathrm{E}+04$ & $1.01 \mathrm{E}+03$ & $9.88 \mathrm{E}+04$ & $2.17 \mathrm{E}+05$ & $2.55 \mathrm{E}+05$ & $4.72 \mathrm{E}+04$ \\
\hline 241-SX-113 & Supernatant & $0.00 \mathrm{E}+00$ & $0.00 \mathrm{E}+00$ & $0.00 \mathrm{E}+00$ & $0.00 \mathrm{E}+00$ & $0.00 \mathrm{E}+00$ & $0.00 \mathrm{E}+00$ \\
\hline & & & & $3.60 \mathrm{E}+04$ & $7.96 \mathrm{E}+04$ & $8.32 \mathrm{E}+04$ & $4.94 \mathrm{E}+03$ \\
\hline & & & & & & $0.00 \mathrm{E}+00$ & $0.00 \mathrm{E}+00$ \\
\hline & & & & & & $2.51 \mathrm{E}+05$ & $4.73 E+04$ \\
\hline & & & & & & $0.00 \mathrm{E}+00$ & +00 \\
\hline & & 6.071 & $2.98 \mathrm{E}+03$ & $3.60 \mathrm{E}+04$ & $7.96 \mathrm{E}+04$ & $8.32 \mathrm{E}+04$ & $4.94 \mathrm{E}+03$ \\
\hline & uperr & 4.811 & $0.00 \mathrm{E}+00$ & $0.00 \mathrm{E}+00$ & $0.00 \mathrm{E}+00$ & $0.00 \mathrm{E}+00$ & $0.00 \mathrm{E}+00$ \\
\hline & & +04 & $4.04 \mathrm{E}+03$ & $1.22 \mathrm{E}+05$ & $1.89 \mathrm{E}+05$ & $2.37 \mathrm{E}+05$ & $3.77 \mathrm{E}+04$ \\
\hline $241-\mathrm{T}-102$ & upern & $6.25 \mathrm{E}+01$ & $4.50 \mathrm{E}+02$ & $2.29 \mathrm{E}+04$ & $1.09 \mathrm{E}+05$ & $6.46 \mathrm{E}+04$ & $8.46 \mathrm{E}+03$ \\
\hline $241-T-102$ & Solid & $6.25 \mathrm{E}+01$ & $4.50 \mathrm{E}+02$ & $2.29 \mathrm{E}+04$ & $1.09 \mathrm{E}+05$ & $6.46 \mathrm{E}+04$ & $8.46 \mathrm{E}+03$ \\
\hline $241-T-103$ & Supernatant & $1.58 \mathrm{E}+02$ & $5.55 \mathrm{E}+03$ & $3.96 \mathrm{E}+04$ & $7.80 \mathrm{E}+04$ & $9.53 \mathrm{E}+04$ & $1.04 \mathrm{E}+04$ \\
\hline $241-T-103$ & Solid & $1.58 \mathrm{E}+02$ & $5.55 \mathrm{E}+03$ & $3.96 \mathrm{E}+04$ & $7.80 \mathrm{E}+04$ & $9.53 \mathrm{E}+04$ & $1.04 \mathrm{E}+04$ \\
\hline $241-\mathrm{T}-104$ & Supernatant & $0.00 \mathrm{E}+00$ & $0.00 \mathrm{E}+00$ & $0.00 \mathrm{E}+00$ & $0.00 \mathrm{E}+00$ & $0.00 \mathrm{E}+00$ & $0.00 \mathrm{E}+00$ \\
\hline $241-T-104$ & Solid & $1.70 \mathrm{E}+04$ & $2.98 \mathrm{E}+03$ & $3.60 \mathrm{E}+04$ & $7.96 \mathrm{E}+04$ & $8.32 \mathrm{E}+04$ & $4.94 \mathrm{E}+03$ \\
\hline
\end{tabular}


Table A-1. Tank Waste Chemical Concentrations.

\begin{tabular}{|c|c|c|c|c|c|c|c|}
\hline Tank & Layer & $\begin{array}{c}\mathbf{O H}^{-} \\
(\mu \mathrm{g} / \mathbf{m L})\end{array}$ & $\begin{array}{c}\text { TOC } \\
(\mu \mathrm{g} / \mathrm{mL})\end{array}$ & $\begin{array}{c}\mathrm{NO}_{2}^{-} \\
(\mu \mathrm{g} / \mathrm{mL})\end{array}$ & $\begin{array}{c}\mathrm{NO}_{3}^{-} \\
(\mu \mathrm{g} / \mathrm{mL})\end{array}$ & $\begin{array}{c}\mathrm{Na}^{+} \\
(\mu \mathrm{g} / \mathrm{mL})\end{array}$ & $\begin{array}{c}\mathbf{A l}^{+3} \\
(\mu \mathrm{g} / \mathrm{mL})\end{array}$ \\
\hline 241-T-105 & Supernatant & $0.00 \mathrm{E}+00$ & $0.00 \mathrm{E}+00$ & $0.00 \mathrm{E}+00$ & $0.00 \mathrm{E}+00$ & $0.00 \mathrm{E}+00$ & $0.00 \mathrm{E}+00$ \\
\hline 241-T-105 & Solid & $1.70 \mathrm{E}+04$ & $2.98 \mathrm{E}+03$ & $5.42 \mathrm{E}+04$ & $3.71 \mathrm{E}+04$ & $8.84 \mathrm{E}+04$ & $3.62 \mathrm{E}+02$ \\
\hline 241-T-106 & Supernatant & $0.00 \mathrm{E}+00$ & $0.00 \mathrm{E}+00$ & $0.00 \mathrm{E}+00$ & $0.00 \mathrm{E}+00$ & $0.00 \mathrm{E}+00$ & $0.00 \mathrm{E}+00$ \\
\hline $241-\mathrm{T}-106$ & Solid & $1.70 \mathrm{E}+04$ & $2.98 \mathrm{E}+03$ & $3.60 \mathrm{E}+04$ & $7.96 \mathrm{E}+04$ & $8.32 E+04$ & $4.94 \mathrm{E}+03$ \\
\hline 241-T-107 & Supernatant & $0.00 \mathrm{E}+00$ & $0.00 \mathrm{E}+00$ & $0.00 \mathrm{E}+00$ & $0.00 \mathrm{E}+00$ & $0.00 \mathrm{E}+00$ & $0.00 \mathrm{E}+00$ \\
\hline 241-T-107 & Solid & $1.70 \mathrm{E}+04$ & $2.98 \mathrm{E}+03$ & $3.60 \mathrm{E}+04$ & $7.96 \mathrm{E}+04$ & $8.32 E+04$ & $4.94 \mathrm{E}+03$ \\
\hline 241-T-108 & Supernatant & $2.07 \mathrm{E}+03$ & $0.00 \mathrm{E}+00$ & $0.00 \mathrm{E}+00$ & $0.00 \mathrm{E}+00$ & $0.00 \mathrm{E}+00$ & $0.00 \mathrm{E}+00$ \\
\hline 241-T-108 & Solid & $2.07 \mathrm{E}+03$ & $3.38 \mathrm{E}+00$ & $8.50 \mathrm{E}+03$ & $2.17 \mathrm{E}+05$ & $1.13 \mathrm{E}+05$ & $3.61 \mathrm{E}+02$ \\
\hline 241-T-109 & Supernatant & $6.44 \mathrm{E}+00$ & $0.00 \mathrm{E}+00$ & $0.00 \mathrm{E}+00$ & $0.00 \mathrm{E}+00$ & $0.00 \mathrm{E}+00$ & $0.00 \mathrm{E}+00$ \\
\hline 241-T-109 & Solid & $6.44 \mathrm{E}+00$ & $3.37 \mathrm{E}+00$ & $8.50 \mathrm{E}+03$ & $2.17 \mathrm{E}+05$ & $1.13 \mathrm{E}+05$ & $3.61 \mathrm{E}+02$ \\
\hline $241-\mathrm{T}-110$ & Supernatant & $2.27 \mathrm{E}-02$ & $4.50 \mathrm{E}+01$ & $5.90 \mathrm{E}+01$ & $1.83 \mathrm{E}+04$ & $3.70 \mathrm{E}+04$ & $0.00 \mathrm{E}+00$ \\
\hline $241-T-110$ & Solid & $2.27 \mathrm{E}-02$ & $4.50 \mathrm{E}+01$ & $5.90 \mathrm{E}+01$ & $1.83 \mathrm{E}+04$ & $3.70 \mathrm{E}+04$ & $0.00 \mathrm{E}+00$ \\
\hline 241-T-111 & Superna & $0.00 \mathrm{E}+00$ & $0.00 \mathrm{E}+00$ & $0.00 \mathrm{E}+00$ & $0.00 \mathrm{E}+00$ & $0.00 \mathrm{E}+00$ & $0.00 \mathrm{E}+00$ \\
\hline 241-T-111 & & $1.70 \mathrm{E}+04$ & $2.98 \mathrm{E}+03$ & $3.60 \mathrm{E}+04$ & $7.96 \mathrm{E}+04$ & $8.32 \mathrm{E}+04$ & $4.94 \mathrm{E}+03$ \\
\hline $241-\mathrm{T}-112$ & Supernatant & $2.11 \mathrm{E}+04$ & $1.61 \mathrm{E}+03$ & $3.88 \mathrm{E}+04$ & $2.33 \mathrm{E}+04$ & $5.72 \mathrm{E}+04$ & $5.04 \mathrm{E}+00$ \\
\hline $241-T-112$ & Solid & $2.11 \mathrm{E}+04$ & $1.61 \mathrm{E}+03$ & $3.88 \mathrm{E}+04$ & $2.33 \mathrm{E}+04$ & $5.72 \mathrm{E}+04$ & $5.04 \mathrm{E}+00$ \\
\hline 241-T-201 & Supernatant & $1.05 \mathrm{E}+03$ & $7.78 \mathrm{E}+01$ & $3.35 \mathrm{E}+02$ & $4.61 \mathrm{E}+04$ & $2.51 \mathrm{E}+04$ & $0.00 \mathrm{E}+00$ \\
\hline $241-T-201$ & Solid & $1.05 E+03$ & $7.78 \mathrm{E}+01$ & $3.35 \mathrm{E}+02$ & $4.61 \mathrm{E}+04$ & $2.51 \mathrm{E}+04$ & $0.00 \mathrm{E}+00$ \\
\hline $241-\mathrm{T}-202$ & Supernatant & $0.00 \mathrm{E}+00$ & $0.00 \mathrm{E}+00$ & $0.00 \mathrm{E}+00$ & $0.00 \mathrm{E}+00$ & $0.00 \mathrm{E}+00$ & $0.00 \mathrm{E}+00$ \\
\hline $241-T-202$ & Solid & $1.70 \mathrm{E}+04$ & $2.98 \mathrm{E}+03$ & $3.60 \mathrm{E}+04$ & $7.96 \mathrm{E}+04$ & $8.32 \mathrm{E}+04$ & $4.94 \mathrm{E}+03$ \\
\hline $241-\mathrm{T}-203$ & Supernatant & $0.00 \mathrm{E}+00$ & $0.00 \mathrm{E}+00$ & $0.00 \mathrm{E}+00$ & $0.00 \mathrm{E}+00$ & $0.00 \mathrm{E}+00$ & $0.00 \mathrm{E}+00$ \\
\hline 241-T-203 & & $1.70 \mathrm{E}+04$ & $2.98 \mathrm{E}+03$ & $3.60 \mathrm{E}+04$ & $7.96 \mathrm{E}+04$ & $8.32 \mathrm{E}+04$ & $4.94 \mathrm{E}+03$ \\
\hline 241-T-204 & Supe & +00 & 0.00 & 0.00 & $0.00 \mathrm{E}+00$ & $0.00 \mathrm{E}+00$ & $\mathrm{E}+00$ \\
\hline 241-T-204 & & +04 & 2.98 & 3.60 & $7.96 \mathrm{E}+04$ & $8.32 \mathrm{E}+04$ & $\mathrm{E}+03$ \\
\hline 241-TX-101 & Supernatant & $4.81 \mathrm{E}+04$ & $0.00 \mathrm{E}+00$ & $0.00 \mathrm{E}+00$ & $0.00 \mathrm{E}+00$ & $0.00 \mathrm{E}+00$ & $0 \mathrm{E}+00$ \\
\hline 241-TX-101 & Solid & $4.81 \mathrm{E}+04$ & $4.03 E+03$ & $1.22 \mathrm{E}+05$ & $1.90 \mathrm{E}+05$ & $2.36 \mathrm{E}+05$ & $3.78 \mathrm{E}+04$ \\
\hline 241-TX-102 & Supernatant & $4.81 \mathrm{E}+04$ & 0.00 & $\mathrm{E}+00$ & $0.00 \mathrm{E}+00$ & $0.00 \mathrm{E}+00$ & $0.00 \mathrm{E}+00$ \\
\hline 241-TX-102 & Solid & $4.81 E+04$ & $4.03 E+03$ & $1.22 \mathrm{E}+05$ & $1.90 \mathrm{E}+05$ & $2.36 \mathrm{E}+05$ & $3.78 \mathrm{E}+04$ \\
\hline 241-TX-103 & Supernatant & $5.72 \mathrm{E}+02$ & $0.00 \mathrm{E}+00$ & $0.00 \mathrm{E}+00$ & $0.00 \mathrm{E}+00$ & $0.00 \mathrm{E}+00$ & $0.00 \mathrm{E}+00$ \\
\hline 241-TX-103 & Solid & $5.72 \mathrm{E}+02$ & $3.88 \mathrm{E}+03$ & $1.18 E+05$ & $1.91 \mathrm{E}+05$ & $2.32 \mathrm{E}+05$ & $3.65 \mathrm{E}+04$ \\
\hline 241-TX-104 & Supernatant & $4.51 E+04$ & $1.36 \mathrm{E}+03$ & $9.42 \mathrm{E}+04$ & $3.54 \mathrm{E}+05$ & $2.06 \mathrm{E}+05$ & $3.19 \mathrm{E}+03$ \\
\hline 241-TX-104 & & $4.51 \mathrm{E}+04$ & $\overline{E+03}$ & $8+04$ & $3.53 \mathrm{E}+05$ & $2.05 \mathrm{E}+05$ & $3.19 \mathrm{E}+03$ \\
\hline $241-T$ & natant & $4.81 \mathrm{E}+04$ & 0.00 & 0.00 & $0.00 \mathrm{E}+00$ & $0.00 \mathrm{E}+00$ & $\overline{\mathrm{E}+00}$ \\
\hline 241-TX-105 & lid & $4.81 \mathrm{E}+04$ & $4.03 \mathrm{E}+03$ & $1.22 \mathrm{E}+05$ & $1.90 \mathrm{E}+05$ & $2.36 \mathrm{E}+05$ & $3.78 \mathrm{E}+04$ \\
\hline 241-TX-106 & Supernatant & $4.81 \mathrm{E}+04$ & $0.00 \mathrm{E}+00$ & $0.00 \mathrm{E}+00$ & $0.00 \mathrm{E}+00$ & $0.00 \mathrm{E}+00$ & $0.00 \mathrm{E}+00$ \\
\hline 241-TX-106 & Solid & $4.81 \mathrm{E}+04$ & $4.03 \mathrm{E}+03$ & $1.22 \mathrm{E}+05$ & $1.90 \mathrm{E}+05$ & $2.36 \mathrm{E}+05$ & $3.78 \mathrm{E}+04$ \\
\hline 241-TX-107 & Supernatant & $3.52 \mathrm{E}+04$ & $0.00 \mathrm{E}+00$ & $0.00 \mathrm{E}+00$ & $0.00 \mathrm{E}+00$ & $0.00 \mathrm{E}+00$ & $0.00 \mathrm{E}+00$ \\
\hline 241-TX-107 & Solid & $3.52 \mathrm{E}+04$ & $3.22 \mathrm{E}+03$ & $1.16 \mathrm{E}+05$ & $1.97 \mathrm{E}+05$ & $2.40 \mathrm{E}+05$ & $4.03 E+04$ \\
\hline 241-TX-108 & Supernatant & $4.81 \mathrm{E}+04$ & $0.00 \mathrm{E}+00$ & $0.00 \mathrm{E}+00$ & $0.00 \mathrm{E}+00$ & $0.00 \mathrm{E}+00$ & $0.00 \mathrm{E}+00$ \\
\hline 241-TX-108 & Solid & $4.81 \mathrm{E}+04$ & $4.03 E+03$ & $1.22 \mathrm{E}+05$ & $1.90 \mathrm{E}+05$ & $2.36 \mathrm{E}+05$ & $3.78 \mathrm{E}+04$ \\
\hline 241-TX-109 & Supernatant & $0.00 \mathrm{E}+00$ & $0.00 \mathrm{E}+00$ & $0.00 \mathrm{E}+00$ & $0.00 \mathrm{E}+00$ & $0.00 \mathrm{E}+00$ & $0.00 \mathrm{E}+00$ \\
\hline 241-TX-109 & Solid & $1.70 \mathrm{E}+04$ & $2.98 \mathrm{E}+03$ & $3.60 \mathrm{E}+04$ & $7.96 \mathrm{E}+04$ & $8.32 E+04$ & $4.94 \mathrm{E}+03$ \\
\hline 241-TX-110 & Supernatant & $4.81 \mathrm{E}+04$ & $0.00 \mathrm{E}+00$ & $0.00 \mathrm{E}+00$ & $0.00 \mathrm{E}+00$ & $0.00 \mathrm{E}+00$ & $0.00 \mathrm{E}+00$ \\
\hline 241-TX-110 & & $4.81 \mathrm{E}+04$ & $4.02 \mathrm{E}+03$ & $1.22 \mathrm{E}+05$ & $1.90 \mathrm{E}+05$ & $2.37 \mathrm{E}+05$ & $3.78 \mathrm{E}+04$ \\
\hline 241-TX-111 & Supernatant & $4.81 E+04$ & $0.00 \mathrm{E}+00$ & $0.00 \mathrm{E}+00$ & $0.00 \mathrm{E}+00$ & $0.00 \mathrm{E}+00$ & $0.00 \mathrm{E}+00$ \\
\hline 241-TX-111 & Solid & $4.81 \mathrm{E}+04$ & $4.03 E+03$ & $1.22 \mathrm{E}+05$ & $1.90 \mathrm{E}+05$ & $2.36 \mathrm{E}+05$ & $3.78 \mathrm{E}+04$ \\
\hline 241-TX-112 & Supernatant & $4.14 \mathrm{E}+04$ & $0.00 \mathrm{E}+00$ & $0.00 \mathrm{E}+00$ & $0.00 \mathrm{E}+00$ & $0.00 \mathrm{E}+00$ & $0.00 \mathrm{E}+00$ \\
\hline 241-TX-112 & Solid & $4.14 E+04$ & $3.47 \mathrm{E}+03$ & $1.06 \mathrm{E}+05$ & $1.94 \mathrm{E}+05$ & $2.18 \mathrm{E}+05$ & $3.26 \mathrm{E}+04$ \\
\hline 241-TX-113 & Supernatant & $4.81 \mathrm{E}+04$ & $0.00 \mathrm{E}+00$ & $0.00 \mathrm{E}+00$ & $0.00 \mathrm{E}+00$ & $0.00 \mathrm{E}+00$ & $0.00 \mathrm{E}+00$ \\
\hline 241-TX-113 & Solid & $4.81 \mathrm{E}+04$ & $4.03 E+03$ & $1.22 \mathrm{E}+05$ & $1.90 \mathrm{E}+05$ & $2.36 \mathrm{E}+05$ & $3.78 E+04$ \\
\hline
\end{tabular}


Table A-1. Tank Waste Chemical Concentrations.

\begin{tabular}{|c|c|c|c|c|c|c|c|}
\hline Tank & Layer & $\begin{array}{c}\mathrm{OH}^{-} \\
(\mu \mathrm{g} / \mathrm{mL})\end{array}$ & $\begin{array}{c}\text { TOC } \\
(\mu \mathrm{g} / \mathrm{mL})\end{array}$ & $\begin{array}{c}\mathrm{NO}_{2}^{-} \\
(\mu \mathrm{g} / \mathrm{mL})\end{array}$ & $\begin{array}{c}\mathrm{NO}_{3}^{-} \\
(\mu \mathrm{g} / \mathrm{mL}) \\
\end{array}$ & $\begin{array}{c}\mathrm{Na}^{+} \\
(\mu \mathrm{g} / \mathrm{mL})\end{array}$ & $\begin{array}{c}\mathbf{A l}^{+3} \\
(\mu \mathrm{g} / \mathrm{mL})\end{array}$ \\
\hline 241-TX-114 & Supernatant & $1.82 \mathrm{E}+04$ & $0.00 \mathrm{E}+00$ & $0.00 \mathrm{E}+00$ & $0.00 \mathrm{E}+00$ & $0.00 \mathrm{E}+00$ & $0.00 \mathrm{E}+00$ \\
\hline 241-TX-114 & Solid & $1.82 \mathrm{E}+04$ & $1.53 \mathrm{E}+03$ & $5.15 \mathrm{E}+04$ & $2.07 \mathrm{E}+05$ & $1.60 \mathrm{E}+05$ & $1.45 \mathrm{E}+04$ \\
\hline 241-TX-115 & Supernatant & $4.81 \mathrm{E}+04$ & $0.00 \mathrm{E}+00$ & $0.00 \mathrm{E}+00$ & $0.00 \mathrm{E}+00$ & $0.00 \mathrm{E}+00$ & $0.00 \mathrm{E}+00$ \\
\hline 241-TX-115 & Solid & $4.81 \mathrm{E}+04$ & $4.03 \mathrm{E}+03$ & $1.22 \mathrm{E}+05$ & $1.90 \mathrm{E}+05$ & $2.36 \mathrm{E}+05$ & $3.78 \mathrm{E}+04$ \\
\hline 241-TX-116 & Supernatant & $2.54 \mathrm{E}+03$ & $0.00 \mathrm{E}+00$ & $0.00 \mathrm{E}+00$ & $0.00 \mathrm{E}+00$ & $0.00 \mathrm{E}+00$ & $0.00 \mathrm{E}+00$ \\
\hline 241-TX-116 & Solid & $2.54 \mathrm{E}+03$ & $3.19 \mathrm{E}+02$ & $1.05 \mathrm{E}+04$ & $3.79 \mathrm{E}+05$ & $1.51 \mathrm{E}+05$ & $8.79 E+00$ \\
\hline 241-TX-117 & Supernatant & $9.91 \mathrm{E}-01$ & $0.00 \mathrm{E}+00$ & $0.00 \mathrm{E}+00$ & $0.00 \mathrm{E}+00$ & $0.00 \mathrm{E}+00$ & $0.00 \mathrm{E}+00$ \\
\hline 241-TX-117 & Solid & $9.91 \mathrm{E}-01$ & $3.38 \mathrm{E}+00$ & $8.50 \mathrm{E}+03$ & $2.17 \mathrm{E}+05$ & $1.13 \mathrm{E}+05$ & $3.60 \mathrm{E}+02$ \\
\hline 241-TX-118 & Supernatant & $4.81 \mathrm{E}+04$ & $0.00 \mathrm{E}+00$ & $0.00 \mathrm{E}+00$ & $0.00 \mathrm{E}+00$ & $0.00 \mathrm{E}+00$ & $0.00 \mathrm{E}+00$ \\
\hline 241-TX-118 & Solid & $4.81 \mathrm{E}+04$ & $4.03 E+03$ & $1.22 \mathrm{E}+05$ & $1.90 \mathrm{E}+05$ & $2.36 \mathrm{E}+05$ & $3.78 \mathrm{E}+04$ \\
\hline 241-TY-101 & Supernatant & $2.32 \mathrm{E}-03$ & $0.00 \mathrm{E}+00$ & $0.00 \mathrm{E}+00$ & $0.00 \mathrm{E}+00$ & $0.00 \mathrm{E}+00$ & $0.00 \mathrm{E}+00$ \\
\hline 241-TY-101 & & $2.32 \mathrm{E}-03$ & $3.38 \mathrm{E}+00$ & $8.47 \mathrm{E}+03$ & $2.17 \mathrm{E}+05$ & $1.13 \mathrm{E}+05$ & $3.61 \mathrm{E}+02$ \\
\hline 241-TY-102 & Supernatant & $2.60 \mathrm{E}+04$ & $0.00 \mathrm{E}+00$ & $0.00 \mathrm{E}+00$ & $0.00 \mathrm{E}+00$ & $0.00 \mathrm{E}+00$ & $0.00 \mathrm{E}+00$ \\
\hline 241-TY-102 & & $2.60 \mathrm{E}+04$ & $2.18 \mathrm{E}+03$ & $6.98 \mathrm{E}+04$ & $2.02 \mathrm{E}+05$ & $1.79 \mathrm{E}+05$ & $2.06 \mathrm{E}+04$ \\
\hline $241-\mathrm{T}$ & $\tan t$ & $4.81 \mathrm{~F}$ & $E+00$ & $E+00$ & 0.00 & $0.00 \mathrm{E}+00$ & $0.00 \mathrm{E}+00$ \\
\hline $241-T$ & & $4.81 \mathrm{E}$ & +03 & $\mathrm{E}+05$ & +05 & $2.36 \mathrm{E}+05$ & $3.78 \mathrm{E}+04$ \\
\hline $241-\mathrm{T}$ & $\tan t$ & 2.381 & +03 & $\mathrm{E}+04$ & $E+04$ & $8.34 \mathrm{E}+04$ & $5.70 \mathrm{E}+02$ \\
\hline $241-T$ & & 2.381 & $\mathrm{E}+03$ & $\mathrm{E}+04$ & $9.90 \mathrm{E}+04$ & $8.34 \mathrm{E}+04$ & $5.70 \mathrm{E}+02$ \\
\hline 241-TY-105 & Supe & $0.00 \mathrm{E}$ & $0.00 \mathrm{E}+00$ & $0.00 \mathrm{E}+00$ & $0.00 \mathrm{E}+00$ & $0.00 \mathrm{E}+00$ & $0.00 \mathrm{E}+00$ \\
\hline 241-TY-105 & & $1.70 \mathrm{E}$ & $2.98 \mathrm{E}+03$ & $3.60 \mathrm{E}+04$ & $\mathrm{E}+04$ & $8.32 E+04$ & $4.94 \mathrm{E}+03$ \\
\hline 241-TY-106 & Supernatant & $0.00 \mathrm{E}+00$ & $0.00 \mathrm{E}+00$ & $0.00 \mathrm{E}+00$ & $0.00 \mathrm{E}+00$ & $0.00 \mathrm{E}+00$ & $0.00 \mathrm{E}+00$ \\
\hline 241-TY-106 & Solid & $1.70 \mathrm{E}+04$ & $2.98 \mathrm{E}+03$ & $3.60 \mathrm{E}+04$ & $7.96 \mathrm{E}+04$ & $8.32 \mathrm{E}+04$ & $4.94 \mathrm{E}+03$ \\
\hline 241-U-101 & Supernatant & $0.00 \mathrm{E}+00$ & $0.00 \mathrm{E}+00$ & $0.00 \mathrm{E}+00$ & $0.00 \mathrm{E}+00$ & $0.00 \mathrm{E}+00$ & $0.00 \mathrm{E}+00$ \\
\hline & & $4.91 \mathrm{E}+04$ & $2.98 E+03$ & $3.60 \mathrm{E}+04$ & $7.96 \mathrm{E}+04$ & $8.32 \mathrm{E}+04$ & $4.94 \mathrm{E}+03$ \\
\hline & Supe & $2.93 \mathrm{I}$ & +04 & +05 & $E+05$ & $2.23 E+05$ & $3 E+04$ \\
\hline & & 04 & +04 & +05 & +05 & $2.23 \mathrm{E}+05$ & $E+04$ \\
\hline 241 & 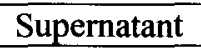 & 04 & +04 & +05 & +05 & $2.31 \mathrm{E}+05$ & $E+04$ \\
\hline 241 & & 3.08 & +04 & +05 & $8+05$ & $2.30 \mathrm{E}+05$ & $E+04$ \\
\hline 24 & Supe & $0.00 \mathrm{E}+00$ & $8+00$ & $\mathrm{E}+00$ & $\mathrm{E}+00$ & $0.00 \mathrm{E}+00$ & $0 \mathrm{E}+00$ \\
\hline & & 4.91 & +03 & +05 & $E+05$ & $2.03 \mathrm{E}+05$ & $1 \mathrm{E}+04$ \\
\hline $241-U-105$ & Supernatant & $2.09 \mathrm{E}+04$ & $0.00 \mathrm{E}+00$ & $0.00 \mathrm{E}+00$ & $0.00 \mathrm{E}+00$ & $0.00 \mathrm{E}+00$ & $0.00 \mathrm{E}+00$ \\
\hline $241-U-105$ & Solid & $2.09 \mathrm{E}+04$ & $1.64 \mathrm{E}+04$ & $1.08 \mathrm{E}+05$ & $1.81 \mathrm{E}+05$ & $2.27 E+05$ & $2.98 \mathrm{E}+04$ \\
\hline $241-U-106$ & Supernatant & $5.11 \mathrm{E}+03$ & $3.38 \mathrm{E}+04$ & $9.90 \mathrm{E}+04$ & $2.48 \mathrm{E}+05$ & $2.23 \mathrm{E}+05$ & $1.20 \mathrm{E}+04$ \\
\hline 241-U-106 & Solid & $5.11 \mathrm{E}+03$ & $3.38 \mathrm{E}+04$ & $9.87 \mathrm{E}+04$ & $2.49 \mathrm{E}+05$ & $2.23 \mathrm{E}+05$ & $1.20 \mathrm{E}+04$ \\
\hline & Supernatant & $3.13 \mathrm{E}+04$ & $0.00 \mathrm{E}+00$ & $0.00 \mathrm{E}+00$ & $0.00 \mathrm{E}+00$ & $0.00 \mathrm{E}+00$ & $0.00 \mathrm{E}+00$ \\
\hline & & & & $1.28 \mathrm{E}+05$ & & $2.05 E+05$ & $4.10 \mathrm{E}+04$ \\
\hline & Sur & & +00 & $E+00$ & +00 & $0.00 \mathrm{E}+00$ & $0 \mathrm{E}+00$ \\
\hline 241 & & 4.92 & $\mathrm{E}+03$ & +05 & $\mathrm{E}+05$ & $2.28 \mathrm{E}+05$ & $5+04$ \\
\hline 241-L & $\operatorname{atan}$ & 4.421 & $0.00 \mathrm{E}+00$ & $0.00 \mathrm{E}+00$ & $0.00 \mathrm{E}+00$ & $0.00 \mathrm{E}+00$ & $0.00 \mathrm{E}+00$ \\
\hline 241-U-109 & & $4.42 \mathrm{E}+04$ & $6.53 \mathrm{E}+03$ & $1.28 \mathrm{E}+05$ & $1.85 \mathrm{E}+05$ & $2.28 \mathrm{E}+05$ & $2.92 \mathrm{E}+04$ \\
\hline 241-U-110 & upernatant & $0.00 \mathrm{E}+00$ & $0.00 \mathrm{E}+00$ & $0.00 \mathrm{E}+00$ & $0.00 \mathrm{E}+00$ & $0.00 \mathrm{E}+00$ & $0.00 \mathrm{E}+00$ \\
\hline 241-U-1 & & $4.91 \mathrm{E}+04$ & $2.98 \mathrm{E}+03$ & $3.60 \mathrm{E}+04$ & $7.96 \mathrm{E}+04$ & $8.32 E+04$ & $4.94 \mathrm{E}+03$ \\
\hline 241-U-111 & Supernatant & $3.39 \mathrm{E}+04$ & $0.00 \mathrm{E}+00$ & $0.00 \mathrm{E}+00$ & $0.00 \mathrm{E}+00$ & $0.00 \mathrm{E}+00$ & $0.00 \mathrm{E}+00$ \\
\hline $241-\mathrm{U}-111$ & Solid & $3.39 \mathrm{E}+04$ & $5.41 \mathrm{E}+03$ & $1.20 \mathrm{E}+05$ & $1.67 \mathrm{E}+05$ & $2.39 \mathrm{E}+05$ & $3.66 \mathrm{E}+04$ \\
\hline $241-\mathrm{U}-112$ & Supernatant & $0.00 \mathrm{E}+00$ & $0.00 \mathrm{E}+00$ & $0.00 \mathrm{E}+00$ & $0.00 \mathrm{E}+00$ & $0.00 \mathrm{E}+00$ & $0.00 \mathrm{E}+00$ \\
\hline $241-\mathrm{U}-112$ & Solid & $4.91 \mathrm{E}+04$ & $2.98 \mathrm{E}+03$ & $3.60 \mathrm{E}+04$ & $7.96 \mathrm{E}+04$ & $8.32 \mathrm{E}+04$ & $4.94 \mathrm{E}+03$ \\
\hline $241-U-201$ & Supernatant & $1.26 \mathrm{E}+04$ & $0.00 \mathrm{E}+00$ & $2.07 \mathrm{E}+04$ & $1.43 \mathrm{E}+05$ & $1.16 \mathrm{E}+05$ & $1.41 \mathrm{E}+02$ \\
\hline $241-U-201$ & Solid & $1.26 \mathrm{E}+04$ & $0.00 \mathrm{E}+00$ & $2.07 \mathrm{E}+04$ & $1.43 \mathrm{E}+05$ & $1.16 \mathrm{E}+05$ & $1.41 \mathrm{E}+02$ \\
\hline 241-U-202 & Supernatant & $1.79 \mathrm{E}+04$ & $1.41 \mathrm{E}+02$ & $2.45 \mathrm{E}+04$ & $1.52 \mathrm{E}+05$ & $1.20 \mathrm{E}+05$ & $2.39 \mathrm{E}+02$ \\
\hline $241-U-202$ & Solid & $1.79 \mathrm{E}+04$ & $1.41 \mathrm{E}+02$ & $2.45 \mathrm{E}+04$ & $1.52 \mathrm{E}+05$ & $1.20 \mathrm{E}+05$ & $2.39 \mathrm{E}+02$ \\
\hline
\end{tabular}


Table A-1. Tank Waste Chemical Concentrations.

\begin{tabular}{|c|c|c|c|c|c|c|c|}
\hline Tank & Layer & $\begin{array}{c}\mathbf{O H}^{-} \\
(\mu \mathrm{g} / \mathbf{m L})\end{array}$ & $\begin{array}{c}\mathbf{T O C} \\
(\mu \mathrm{g} / \mathbf{m L})\end{array}$ & $\begin{array}{c}\mathbf{N O}_{2}^{-} \\
(\mu \mathrm{g} / \mathbf{m L})\end{array}$ & $\begin{array}{c}\mathbf{N O}_{\mathbf{3}}^{-} \\
(\mu \mathrm{g} / \mathbf{m L})\end{array}$ & $\begin{array}{c}\mathbf{N a}^{+} \\
(\mu \mathrm{g} / \mathbf{m L})\end{array}$ & $\begin{array}{c}\mathbf{A l}^{+3} \\
(\mu \mathrm{g} / \mathbf{m L})\end{array}$ \\
\hline $241-\mathrm{U}-203$ & Supernatant & $4.75 \mathrm{E}+03$ & $0.00 \mathrm{E}+00$ & $1.94 \mathrm{E}+04$ & $1.44 \mathrm{E}+05$ & $1.00 \mathrm{E}+05$ & $1.88 \mathrm{E}+02$ \\
\hline $241-\mathrm{U}-203$ & Solid & $4.75 \mathrm{E}+03$ & $0.00 \mathrm{E}+00$ & $1.94 \mathrm{E}+04$ & $1.44 \mathrm{E}+05$ & $1.00 \mathrm{E}+05$ & $1.88 \mathrm{E}+02$ \\
\hline $241-\mathrm{U}-204$ & Supernatant & $1.61 \mathrm{E}+03$ & $8.10 \mathrm{E}+01$ & $7.03 \mathrm{E}+03$ & $5.53 \mathrm{E}+04$ & $3.93 \mathrm{E}+04$ & $1.46 \mathrm{E}+01$ \\
\hline $241-\mathrm{U}-204$ & Solid & $1.61 \mathrm{E}+03$ & $8.10 \mathrm{E}+01$ & $7.03 \mathrm{E}+03$ & $5.53 \mathrm{E}+04$ & $3.93 \mathrm{E}+04$ & $1.46 \mathrm{E}+01$ \\
\hline
\end{tabular}

Table A-2. Tank Waste Radionuclide Concentrations.

\begin{tabular}{|c|c|c|c|c|c|c|c|}
\hline Tank & Layer & $\begin{array}{c}{ }^{90} \mathrm{Sr} \\
(\mu \mathrm{Ci} / \mathrm{g})\end{array}$ & $\begin{array}{l}{ }^{241} \mathrm{Am} \\
(\mu \mathrm{Ci} / \mathrm{g})\end{array}$ & $\begin{array}{c}{ }^{240} \mathrm{Pu} \\
(\mu \mathrm{Ci} / \mathrm{g})\end{array}$ & $\begin{array}{c}{ }^{239} \mathrm{Pu} \\
(\mu \mathrm{Ci} / \mathrm{g})\end{array}$ & $\begin{array}{c}{ }^{238} \mathrm{Pu} \\
(\mu \mathrm{Ci} / \mathrm{g})\end{array}$ & $\begin{array}{c}{ }^{137} \mathrm{Cs} \\
(\mu \mathrm{Ci} / \mathrm{g})\end{array}$ \\
\hline 241-AN-101 & Supernatant & $1.21 \mathrm{E}+00$ & $1.62 \mathrm{E}-03$ & $2.38 \mathrm{E}-05$ & $1.42 \mathrm{E}-04$ & $4.29 \mathrm{E}-06$ & $1.77 \mathrm{E}+02$ \\
\hline 241-AN-101 & Solid & $2.23 \mathrm{E}+01$ & $3.54 \mathrm{E}-02$ & $2.31 \mathrm{E}-03$ & $9.97 \mathrm{E}-03$ & $3.97 \mathrm{E}-04$ & $1.67 \mathrm{E}+02$ \\
\hline $241-\mathrm{AN}-102$ & Supernatant & $5.12 \mathrm{E}+01$ & $1.15 \mathrm{E}-01$ & $8.81 \mathrm{E}-04$ & $3.40 \mathrm{E}-03$ & $2.39 \mathrm{E}-04$ & $2.55 \mathrm{E}+02$ \\
\hline 241-AN-102 & Solid & $1.24 \mathrm{E}+02$ & $5.97 \mathrm{E}-01$ & $1.05 \mathrm{E}-02$ & $4.04 \mathrm{E}-02$ & $2.84 \mathrm{E}-03$ & $2.41 \mathrm{E}+02$ \\
\hline $241-\mathrm{AN}-103$ & Supernatant & $1.08 \mathrm{E}-02$ & $4.09 \mathrm{E}-03$ & $4.47 \mathrm{E}-05$ & $1.72 \mathrm{E}-04$ & $1.21 \mathrm{E}-05$ & $3.90 \mathrm{E}+02$ \\
\hline $241-\mathrm{AN}-103$ & Solid & $2.02 \mathrm{E}+00$ & $3.52 \mathrm{E}-03$ & $3.58 \mathrm{E}-04$ & $1.38 \mathrm{E}-03$ & $9.69 \mathrm{E}-05$ & $1.87 \mathrm{E}+02$ \\
\hline 241-AN-104 & Supernatant & $5.32 \mathrm{E}-02$ & $2.50 \mathrm{E}-04$ & $1.34 \mathrm{E}-05$ & $5.16 \mathrm{E}-05$ & $3.64 \mathrm{E}-06$ & $3.20 \mathrm{E}+02$ \\
\hline 241-AN-104 & Solid & $2.61 \mathrm{E}+01$ & $1.89 \mathrm{E}-02$ & $7.43 \mathrm{E}-04$ & $2.86 \mathrm{E}-03$ & $2.01 \mathrm{E}-04$ & $2.74 \mathrm{E}+02$ \\
\hline 241-AN-105 & Supernatant & $2.22 \mathrm{E}-02$ & $1.01 \mathrm{E}-02$ & $1.10 \mathrm{E}-04$ & $4.24 \mathrm{E}-04$ & $2.98 \mathrm{E}-05$ & $1.88 \mathrm{E}+02$ \\
\hline 241-AN-105 & Solid & $8.64 E+00$ & $6.92 \mathrm{E}-03$ & $4.67 \mathrm{E}-04$ & $1.79 \mathrm{E}-03$ & $1.26 \mathrm{E}-04$ & $2.28 \mathrm{E}+02$ \\
\hline $241-\mathrm{AN}-10$ & Supe & $4.30 \mathrm{E}-02$ & $4.73 \mathrm{E}$ & $1.04 \mathrm{E}-04$ & $4.96 \mathrm{E}-04$ & $2.48 \mathrm{E}-05$ & $1.40 \mathrm{E}+01$ \\
\hline $241-x$ & & $9.16 \mathrm{E}+02$ & $1.11 \mathrm{E}$ & $1.31 \mathrm{E}$ & $6.25 \mathrm{E}$ & $1.88 \mathrm{E}$ & $1.35 \mathrm{E}+02$ \\
\hline 241 & & $4.88 \mathrm{E}+01$ & $5.09 \mathrm{E}$ & $8.21 \mathrm{E}-03$ & $3.16 \mathrm{E}-02$ & $2.23 \mathrm{E}-03$ & $1.90 \mathrm{E}+02$ \\
\hline $241-$ & & $1.51 \mathrm{E}+02$ & $9.30 \mathrm{E}$ & $1.43 \mathrm{E}-02$ & $5.49 \mathrm{E}-02$ & $3.86 \mathrm{E}-03$ & $2.13 \mathrm{E}+02$ \\
\hline 241 & upe & $1.85 \mathrm{E}-01$ & $1.11 E-04$ & $8.48 \mathrm{E}-06$ & $4.64 \mathrm{E}-05$ & $9.11 \mathrm{E}-06$ & $4.84 \mathrm{E}+01$ \\
\hline $241-\mathrm{A}$ & Sol & $0.00 \mathrm{E}+00$ & $0.00 \mathrm{E}+00$ & $0.00 \mathrm{E}+00$ & $0.00 \mathrm{E}+00$ & $0.00 \mathrm{E}+00$ & $0.00 \mathrm{E}+00$ \\
\hline 241-AP-102 & Supernatant & $2.57 \mathrm{E}-01$ & $1.26 \mathrm{E}-03$ & $3.83 \mathrm{E}-05$ & $2.26 \mathrm{E}-04$ & $5.71 \mathrm{E}-06$ & $1.60 \mathrm{E}+02$ \\
\hline 241-AP-102 & Solid & $3.34 \mathrm{E}-01$ & $7.65 \mathrm{E}-04$ & $8.86 \mathrm{E}-05$ & $5.26 \mathrm{E}-04$ & $1.31 \mathrm{E}-05$ & $7.65 \mathrm{E}+01$ \\
\hline $241-A$ & upernatant & $1.45 \mathrm{E}+00$ & $5.09 \mathrm{E}-03$ & $1.72 \mathrm{E}-04$ & $1.03 \mathrm{E}-03$ & $1.40 \mathrm{E}-04$ & $1.60 \mathrm{E}+02$ \\
\hline $241-A$ & & $1.47 \mathrm{E}+01$ & $1.65 \mathrm{E}-02$ & $4.31 \mathrm{E}-03$ & $1.65 \mathrm{E}-02$ & $4.55 \mathrm{E}-03$ & $1.85 \mathrm{E}+02$ \\
\hline 241 & matant & $7.49 \mathrm{E}-01$ & $1.90 \mathrm{E}$ & $5.59 \mathrm{E}-$ & $3.31 \mathrm{E}-04$ & $6.30 \mathrm{E}-05$ & $1.70 \mathrm{E}+02$ \\
\hline 241-AP-104 & & $1.76 \mathrm{E}+01$ & $1.95 \mathrm{E}$ & $3.28 \mathrm{E}-03$ & $1.26 \mathrm{E}-02$ & $8.86 \mathrm{E}-04$ & $2.23 \mathrm{E}+02$ \\
\hline 241-AP-10 & $S$ & $6.94 \mathrm{E}-01$ & $4.24 \mathrm{E}$ & $4.81 \mathrm{E}-04$ & $1.84 \mathrm{E}-03$ & $1.60 \mathrm{E}$ & $2.45 \mathrm{E}+02$ \\
\hline 241 & & $1.62 \mathrm{E}+01$ & $5.27 \mathrm{E}$ & $8.89 \mathrm{E}$ & $3.42 \mathrm{E}$ & $2.40 \mathrm{E}-04$ & $2.05 \mathrm{E}+02$ \\
\hline 241 . & ant & 01 & 4.97 & 1.05 & 05 & $1.66 \mathrm{E}-06$ & $1.32 \mathrm{E}+02$ \\
\hline $241-$ & & $0.00 \mathrm{E}+00$ & $0.00 \mathrm{E}$ & $0.00 \mathrm{E}+00$ & $0.00 \mathrm{E}$ & $0.00 \mathrm{E}+00$ & $0.00 \mathrm{E}+00$ \\
\hline 241 & $\tan t$ & $7.68 \mathrm{E}-01$ & $4.08 \mathrm{E}-04$ & $5.43 \mathrm{E}-04$ & $1.99 \mathrm{E}-03$ & $1.85 \mathrm{E}-04$ & $2.74 \mathrm{E}+02$ \\
\hline $241-$ & & $0.00 \mathrm{E}+00$ & $0.00 \mathrm{E}+00$ & $0.00 \mathrm{E}+00$ & $0.00 \mathrm{E}+00$ & $0.00 \mathrm{E}+00$ & $0.00 \mathrm{E}+00$ \\
\hline P-108 & upernatant & $6.53 \mathrm{E}-01$ & $1.33 \mathrm{E}-03$ & $5.38 \mathrm{E}-05$ & $2.82 \mathrm{E}-04$ & $1.71 \mathrm{E}-05$ & $1.33 \mathrm{E}+02$ \\
\hline 241-AP-108 & Solid & $4.20 \mathrm{E}-01$ & 2.17E-04 & 4.27E-05 & $2.11 \mathrm{E}-04$ & $1.53 \mathrm{E}-05$ & $1.17 \mathrm{E}+02$ \\
\hline $241-\mathrm{AW}-101$ & upernatant & $2.31 \mathrm{E}-01$ & $1.84 \mathrm{E}-04$ & 7.27E-05 & $2.80 \mathrm{E}-04$ & $1.96 \mathrm{E}-05$ & $2.34 \mathrm{E}+02$ \\
\hline 241-AW-101 & Solid & $3.34 \mathrm{E}+01$ & $4.75 \mathrm{E}-02$ & $1.16 \mathrm{E}-02$ & $4.45 \mathrm{E}-02$ & $3.13 \mathrm{E}-03$ & $1.94 \mathrm{E}+02$ \\
\hline 241-AW-102 & Supernatant & $2.19 \mathrm{E}-01$ & $1.71 \mathrm{E}-04$ & $1.12 \mathrm{E}-05$ & $6.33 \mathrm{E}-05$ & $1.23 \mathrm{E}-05$ & $4.79 \mathrm{E}+01$ \\
\hline $241-\mathrm{AW}-102$ & Solid & $4.93 \mathrm{E}+02$ & $1.82 \mathrm{E}+00$ & $9.24 \mathrm{E}-02$ & $4.45 \mathrm{E}-01$ & $7.44 \mathrm{E}-03$ & $6.49 \mathrm{E}+01$ \\
\hline 241-AW-103 & Supernatant & $1.64 \mathrm{E}-01$ & $4.29 \mathrm{E}-05$ & $2.07 \mathrm{E}-05$ & $9.74 \mathrm{E}-05$ & $8.53 \mathrm{E}-06$ & $8.37 \mathrm{E}+01$ \\
\hline 241-AW-103 & Solid & $6.22 \mathrm{E}+00$ & $9.76 \mathrm{E}-02$ & $6.14 \mathrm{E}-02$ & $2.17 \mathrm{E}-01$ & $2.08 \mathrm{E}-02$ & $2.52 \mathrm{E}+01$ \\
\hline 241-AW-104 & Supernatant & $1.10 \mathrm{E}+00$ & $1.16 \mathrm{E}-03$ & $1.89 \mathrm{E}-05$ & $1.06 \mathrm{E}-04$ & $3.26 \mathrm{E}-06$ & $1.62 \mathrm{E}+02$ \\
\hline 241-AW-104 & Solid & $4.99 \mathrm{E}-01$ & $6.91 \mathrm{E}-02$ & $1.38 \mathrm{E}-01$ & $4.92 \mathrm{E}-01$ & $4.77 \mathrm{E}-02$ & $1.46 \mathrm{E}+02$ \\
\hline 241-AW-105 & Supernatant & $1.94 \mathrm{E}-02$ & $7.06 \mathrm{E}-05$ & $6.23 \mathrm{E}-05$ & $2.21 \mathrm{E}-04$ & $2.12 \mathrm{E}-05$ & $8.79 E+00$ \\
\hline 241-AW-105 & Solid & $4.38 \mathrm{E}+01$ & $5.56 \mathrm{E}-01$ & $1.63 \mathrm{E}-01$ & $5.81 \mathrm{E}-01$ & $5.60 \mathrm{E}-02$ & $2.66 \mathrm{E}+01$ \\
\hline
\end{tabular}


Table A-2. Tank Waste Radionuclide Concentrations.

\begin{tabular}{|c|c|c|c|c|c|c|c|}
\hline Tank & Layer & $\begin{array}{c}{ }^{90} \mathrm{Sr} \\
(\mu \mathrm{Ci} / \mathrm{g})\end{array}$ & $\begin{array}{l}{ }^{241} \mathrm{Am} \\
(\mu \mathrm{Ci} / \mathrm{g})\end{array}$ & $\begin{array}{c}{ }^{240} \mathrm{Pu} \\
(\mu \mathrm{Ci} / \mathrm{g})\end{array}$ & $\begin{array}{c}{ }^{239} \mathrm{Pu} \\
(\mu \mathrm{Ci} / \mathrm{g})\end{array}$ & $\begin{array}{c}{ }^{238} \mathrm{Pu} \\
(\mu \mathrm{Ci} / \mathrm{g})\end{array}$ & $\begin{array}{c}{ }^{137} \mathrm{Cs} \\
(\mu \mathrm{Ci} / \mathrm{g})\end{array}$ \\
\hline 241-AW-106 & Supernatant & $6.07 \mathrm{E}-01$ & $6.05 \mathrm{E}-04$ & $4.46 \mathrm{E}-04$ & $1.94 \mathrm{E}-03$ & $1.27 \mathrm{E}-04$ & $2.22 \mathrm{E}+02$ \\
\hline 241-AW-106 & Solid & $2.18 \mathrm{E}+01$ & $2.75 \mathrm{E}-02$ & $2.50 \mathrm{E}-02$ & $9.58 \mathrm{E}-02$ & $6.76 \mathrm{E}-03$ & $2.75 E+02$ \\
\hline 241-AY-101 & Supernatant & $6.31 \mathrm{E}-01$ & $3.17 \mathrm{E}-03$ & $6.50 \mathrm{E}-04$ & $2.91 \mathrm{E}-03$ & $1.10 \mathrm{E}-04$ & $1.65 \mathrm{E}+01$ \\
\hline 241-AY-101 & Solid & $2.99 \mathrm{E}+03$ & $2.11 \mathrm{E}+01$ & $3.33 \mathrm{E}-01$ & $1.08 \mathrm{E}+00$ & $8.97 \mathrm{E}-02$ & $1.01 \mathrm{E}+02$ \\
\hline 241-AY-102 & atant & $1.31 \mathrm{E}-01$ & $1.60 \mathrm{E}-04$ & $4.19 \mathrm{E}-05$ & $1.78 \mathrm{E}-04$ & $2.09 \mathrm{E}-05$ & $9.61 \mathrm{E}+01$ \\
\hline $241-\mathrm{AY}-102$ & Solid & $4.63 \mathrm{E}+03$ & $5.45 \mathrm{E}+00$ & $4.05 \mathrm{E}-01$ & $1.76 \mathrm{E}+00$ & $6.52 \mathrm{E}-02$ & $2.50 \mathrm{E}+02$ \\
\hline $241-\mathrm{AZ}-101$ & Superna & $5.82 \mathrm{E}-01$ & $1.49 \mathrm{E}-04$ & $3.06 \mathrm{E}-04$ & $1.09 \mathrm{E}-03$ & $1.07 \mathrm{E}-04$ & $1.05 \mathrm{E}+03$ \\
\hline $241-\mathrm{AZ}-10$ & & $1.53 \mathrm{E}+04$ & $7.76 \mathrm{E}^{+}$ & $1.01 \mathrm{E}+00$ & $3.57 \mathrm{E}+00$ & $3.40 \mathrm{E}-$ & $7.12 \mathrm{E}+02$ \\
\hline $241-\mathrm{AZ}-102$ & in & $1.79 \mathrm{E}+00$ & $9.15 \mathrm{E}$ & $2.00 \mathrm{E}-03$ & $7.31 \mathrm{E}-03$ & $6.86 \mathrm{E}$ & $8.64 \mathrm{E}+02$ \\
\hline $241-\mathrm{AZ}-102$ & & $6.37 \mathrm{E}+03$ & $5.05 \mathrm{E}-$ & $6.16 \mathrm{E}-01$ & $2.34 \mathrm{E}+00$ & $3.38 \mathrm{E}-01$ & $6.04 \mathrm{E}+02$ \\
\hline $241-S$ & atant & $2.05 \mathrm{E}-02$ & $4.60 \mathrm{E}$ & $7.31 \mathrm{E}-06$ & $3.31 \mathrm{E}-05$ & $2.63 \mathrm{E}-06$ & $2.07 \mathrm{E}+01$ \\
\hline 241-SY-101 & & $5.13 \mathrm{E}+01$ & $4.90 \mathrm{E}$ & $9.26 \mathrm{E}-03$ & $4.31 \mathrm{E}-02$ & $8.99 \mathrm{E}-03$ & $1.33 \mathrm{E}+02$ \\
\hline 241-SY-102 & upernatant & $2.70 \mathrm{E}-01$ & $1.58 \mathrm{E}-03$ & $1.77 \mathrm{E}-04$ & $8.19 \mathrm{E}-04$ & $3.01 \mathrm{E}-05$ & $1.82 \mathrm{E}+01$ \\
\hline $241-S Y-102$ & Solid & $1.65 \mathrm{E}+02$ & $1.72 \mathrm{E}+01$ & $9.43 \mathrm{E}-01$ & $4.09 \mathrm{E}+00$ & $1.88 \mathrm{E}-01$ & $9.55 \mathrm{E}+01$ \\
\hline $241-$ & atant & $1.84 \mathrm{E}+00$ & $6.79 \mathrm{E}-03$ & $7.85 \mathrm{E}-06$ & $3.66 \mathrm{E}-05$ & $1.24 \mathrm{E}-06$ & $2.63 E+02$ \\
\hline $241-5$ & & $2.88 \mathrm{E}+01$ & $6.09 \mathrm{E}$ & $1.21 \mathrm{E}-02$ & $5.66 \mathrm{E}-02$ & $1.91 \mathrm{E}-03$ & $1.93 \mathrm{E}+02$ \\
\hline & uper & $0.00 \mathrm{E}+00$ & $0.00 \mathrm{E}+00$ & $0.00 \mathrm{E}+00$ & $0.00 \mathrm{E}+00$ & $0.00 \mathrm{E}+00$ & $0.00 \mathrm{E}+00$ \\
\hline & & $6.22 \mathrm{E}+01$ & $2.13 \mathrm{E}$ & $1.09 \mathrm{E}-02$ & $4.52 \mathrm{E}-02$ & $1.96 \mathrm{E}$ & $1.56 \mathrm{E}+02$ \\
\hline & $\underline{S}$ & $6.42 \mathrm{E}-01$ & & $5.41 \mathrm{E}-04$ & $2.34 \mathrm{E}-03$ & $9.29 \mathrm{E}$ & $2.88 \mathrm{E}+02$ \\
\hline & & $3.04 \mathrm{E}+02$ & & $3.12 \mathrm{E}$ & $1.35 \mathrm{E}$ & $5.35 \mathrm{H}$ & $1.36 \mathrm{E}+02$ \\
\hline & & $16 \mathrm{E}+00$ & 2.20 & $3.35 \mathrm{E}$ & $1.44 \mathrm{E}$ & $5.73 \mathrm{E}$ & $1.63 E+02$ \\
\hline 241 & & $80 \mathrm{E}+01$ & $1.22 \mathrm{E}$ & $2.61 \mathrm{E}-02$ & $1.14 \mathrm{E}-01$ & $4.37 \mathrm{E}$ & $1.30 \mathrm{E}+02$ \\
\hline 24 & ape & $00 \mathrm{E}+00$ & $0.00 \mathrm{E}$ & $0.00 \mathrm{E}+00$ & $0.00 \mathrm{E}+00$ & $0.00 \mathrm{E}+00$ & $0.00 \mathrm{E}+00$ \\
\hline 24 & & $07 \mathrm{E}+04$ & $8.02 \mathrm{E}-$ & $1.48 \mathrm{E}+00$ & $6.76 \mathrm{E}+00$ & $2.17 \mathrm{E}-01$ & $5.61 \mathrm{E}+02$ \\
\hline 241 & uper & $00 \mathrm{E}+00$ & $0.00 \mathrm{E}+00$ & $0.00 \mathrm{E}+00$ & $0.00 \mathrm{E}+00$ & $0.00 \mathrm{E}+00$ & $0.00 \mathrm{E}+00$ \\
\hline $241-$ & $\mathrm{S}$ & $1.22 \mathrm{E}+04$ & $2.61 \mathrm{E}+01$ & $6.35 \mathrm{E}-01$ & $2.60 \mathrm{E}+00$ & $1.18 \mathrm{E}-01$ & $4.58 \mathrm{E}+02$ \\
\hline 241 & atant & $0.00 \mathrm{E}+00$ & $0.00 \mathrm{E}+00$ & $0.00 \mathrm{E}+00$ & $0.00 \mathrm{E}+00$ & $0.00 \mathrm{E}+00$ & $0.00 \mathrm{E}+00$ \\
\hline 241 & & $8.77 \mathrm{E}+02$ & $1.15 \mathrm{E}$ & $4.64 \mathrm{E}-01$ & $2.03 \mathrm{E}+00$ & $7.67 \mathrm{E}-02$ & $1.15 \mathrm{E}+02$ \\
\hline 241 & $\mathrm{~S}$ & $0.00 \mathrm{E}+00$ & $0.00 \mathrm{E}$ & $0.00 \mathrm{E}+00$ & $0.00 \mathrm{E}+00$ & $0.00 \mathrm{E}+00$ & $0.00 \mathrm{E}+00$ \\
\hline & & $8.73 \mathrm{E}+01$ & 1.701 & $1.17 \mathrm{E}-02$ & $5.02 \mathrm{E}-02$ & $2.01 \mathrm{E}-03$ & $1.61 \mathrm{E}+02$ \\
\hline & & & & & $0.00 \mathrm{E}-$ & $0.00 \mathrm{E}$ & $0.00 \mathrm{E}+00$ \\
\hline & & & & & 01 & $7.64 \mathrm{I}$ & $1.26 \mathrm{E}+02$ \\
\hline & & & & & -00 & 00 & $00 \mathrm{E}+00$ \\
\hline & & $\bar{E}+02$ & & $3.66 \mathrm{E}-$ & $1.45 \mathrm{E}-01$ & 6.581 & $1.65 \mathrm{E}+02$ \\
\hline 241 & $s$ & $0.00 \mathrm{E}+00$ & $0.00 \mathrm{E}$ & $0.00 \mathrm{E}+00$ & $0.00 \mathrm{E}+00$ & $0.00 \mathrm{E}+00$ & $0.00 \mathrm{E}+00$ \\
\hline 241 & & $3.71 \mathrm{E}+04$ & $1.88 \mathrm{E}$ & $1.31 \mathrm{E}+00$ & $5.34 \mathrm{E}+00$ & $2.44 \mathrm{E}-01$ & $8.99 \mathrm{E}+02$ \\
\hline & upe & $0.00 \mathrm{E}+00$ & $0.00 \mathrm{E}-$ & $0.00 \mathrm{E}+00$ & $0.00 \mathrm{E}+00$ & $0.00 \mathrm{E}+00$ & $0.00 \mathrm{E}+00$ \\
\hline & d & $2.96 \mathrm{E}+02$ & $2.28 \mathrm{E}+00$ & $1.55 \mathrm{E}-01$ & $5.02 \mathrm{E}-01$ & 4.27E- 02 & $3.16 \mathrm{E}+00$ \\
\hline 241-E & upernatant & $1.53 \mathrm{E}+00$ & $1.23 \mathrm{E}-03$ & $4.69 \mathrm{E}-04$ & $4.14 \mathrm{E}-03$ & $2.98 \mathrm{E}-05$ & $6.46 \mathrm{E}+00$ \\
\hline 102 & Sol & $5.37 \mathrm{E}-01$ & $4.48 \mathrm{E}-04$ & $3.31 \mathrm{E}-03$ & 2.93E-02 & $2.11 \mathrm{E}-04$ & $2.40 \mathrm{E}+00$ \\
\hline 241-E & upernatant & $0.00 \mathrm{E}+00$ & $0.00 \mathrm{E}+00$ & $0.00 \mathrm{E}+00$ & $0.00 \mathrm{E}+00$ & $0.00 \mathrm{E}+00$ & $0.00 \mathrm{E}+00$ \\
\hline 241-B-103 & Solid & $1.27 \mathrm{E}+00$ & $1.39 \mathrm{E}-03$ & $1.70 \mathrm{E}-02$ & $1.50 \mathrm{E}-01$ & $1.08 \mathrm{E}-03$ & $2.42 \mathrm{E}+00$ \\
\hline & Super & $0.00 \mathrm{E}+00$ & $0.00 \mathrm{E}+00$ & $0.00 \mathrm{E}+00$ & $0.00 \mathrm{E}+00$ & $0.00 \mathrm{E}+00$ & $0.00 \mathrm{E}+00$ \\
\hline 241-B-104 & & $1.80 \mathrm{E}+00$ & $2.47 \mathrm{E}-03$ & $4.71 \mathrm{E}-03$ & $4.40 \mathrm{E}-02$ & $2.93 \mathrm{E}-04$ & $6.01 \mathrm{E}+00$ \\
\hline 241-B-105 & Supernatant & $0.00 \mathrm{E}+00$ & $0.00 \mathrm{E}+00$ & $0.00 \mathrm{E}+00$ & $0.00 \mathrm{E}+00$ & $0.00 \mathrm{E}+00$ & $0.00 \mathrm{E}+00$ \\
\hline & & & $7.91 \mathrm{E}-04$ & & $3.43 \mathrm{E}-02$ & $2.45 \mathrm{E}-04$ & $2.13 \mathrm{E}+00$ \\
\hline & & $1.53 \mathrm{E}+00$ & $1.23 \mathrm{E}-$ & $4.68 \mathrm{E}-04$ & $4.15 \mathrm{E}-03$ & $2.98 \mathrm{E}-05$ & $6.45 \mathrm{E}+00$ \\
\hline & & $4.46 \mathrm{E}+01$ & 9.92E- & $3.14 \mathrm{E}-03$ & $2.92 \mathrm{E}-02$ & $1.98 \mathrm{E}-04$ & $1.41 \mathrm{E}+01$ \\
\hline & Supe & $0.00 \mathrm{E}+00$ & $0.00 \mathrm{E}+00$ & $0.00 \mathrm{E}+00$ & $0.00 \mathrm{E}+00$ & $0.00 \mathrm{E}+00$ & $0.00 \mathrm{E}+00$ \\
\hline 241-B-107 & & $2.92 \mathrm{E}+00$ & $4.62 \mathrm{E}-03$ & $4.74 \mathrm{E}-03$ & $4.03 \mathrm{E}-02$ & $3.17 \mathrm{E}-04$ & $1.00 \mathrm{E}+01$ \\
\hline
\end{tabular}


Table A-2. Tank Waste Radionuclide Concentrations.

\begin{tabular}{|c|c|c|c|c|c|c|c|}
\hline Tank & Layer & $\begin{array}{c}{ }^{90} \mathrm{Sr} \\
(\mu \mathrm{Ci} / \mathrm{g})\end{array}$ & $\begin{array}{l}{ }^{241} \mathrm{Am} \\
(\mu \mathrm{Ci} / \mathrm{g})\end{array}$ & $\begin{array}{c}{ }^{240} \mathrm{Pu} \\
(\mu \mathrm{Ci} / \mathrm{g})\end{array}$ & $\begin{array}{c}{ }^{239} \mathrm{Pu} \\
(\mu \mathrm{Ci} / \mathrm{g})\end{array}$ & $\begin{array}{c}{ }^{238} \mathrm{Pu} \\
(\mu \mathrm{Ci} / \mathrm{g})\end{array}$ & $\begin{array}{c}{ }^{137} \mathrm{Cs} \\
(\mu \mathrm{Ci} / \mathrm{g})\end{array}$ \\
\hline $241-\mathrm{B}-108$ & Supernatant & $0.00 \mathrm{E}+00$ & $0.00 \mathrm{E}+00$ & $0.00 \mathrm{E}+00$ & $0.00 \mathrm{E}+00$ & $0.00 \mathrm{E}+00$ & $0.00 \mathrm{E}+00$ \\
\hline 241-B-108 & Solid & $1.41 \mathrm{E}+00$ & $1.90 \mathrm{E}-04$ & $4.83 \mathrm{E}-04$ & $3.29 \mathrm{E}-03$ & $3.79 \mathrm{E}-05$ & $1.15 \mathrm{E}+01$ \\
\hline 241-B-109 & Supernatant & $0.00 \mathrm{E}+00$ & $0.00 \mathrm{E}+00$ & $0.00 \mathrm{E}+00$ & $0.00 \mathrm{E}+00$ & $0.00 \mathrm{E}+00$ & $0.00 \mathrm{E}+00$ \\
\hline 241-B-109 & Solid & $3.95 \mathrm{E}-01$ & $7.82 \mathrm{E}-04$ & $3.33 \mathrm{E}-03$ & $2.47 \mathrm{E}-02$ & $2.46 \mathrm{E}-04$ & $1.36 \mathrm{E}+00$ \\
\hline 241-B-110 & Supernatant & $1.76 \mathrm{E}+00$ & $5.67 \mathrm{E}-03$ & $9.83 \mathrm{E}-04$ & $4.24 \mathrm{E}-03$ & $1.72 \mathrm{E}-04$ & $1.07 \mathrm{E}+01$ \\
\hline $241-\mathrm{B}-110$ & Solid & $7.06 \mathrm{E}+01$ & $7.20 \mathrm{E}-02$ & $1.08 \mathrm{E}-02$ & $1.01 \mathrm{E}-01$ & $3.04 \mathrm{E}-03$ & $9.90 \mathrm{E}+00$ \\
\hline $241-\mathrm{B}-111$ & Supernatant & $1.76 \mathrm{E}+00$ & $5.67 \mathrm{E}-03$ & $9.83 \mathrm{E}-04$ & $4.24 \mathrm{E}-03$ & $1.72 \mathrm{E}-04$ & $1.07 \mathrm{E}+01$ \\
\hline 241-B-111 & Solid & $1.74 \mathrm{E}+02$ & $8.50 \mathrm{E}-02$ & $1.09 \mathrm{E}-02$ & $8.63 \mathrm{E}-02$ & $2.87 \mathrm{E}-03$ & $1.13 \mathrm{E}+02$ \\
\hline $241-B-112$ & Supernatant & $1.38 \mathrm{E}+00$ & $4.48 \mathrm{E}-03$ & $8.01 \mathrm{E}-04$ & 3.34E-03 & $1.25 \mathrm{E}-04$ & $9.27 \mathrm{E}+01$ \\
\hline 241-B-112 & Solid & $3.84 E+00$ & $1.16 \mathrm{E}-03$ & $3.55 \mathrm{E}-04$ & $2.52 \mathrm{E}-03$ & $3.74 \mathrm{E}-05$ & $3.14 \mathrm{E}+01$ \\
\hline 241-B-201 & Supernatant & $0.00 \mathrm{E}+00$ & $0.00 \mathrm{E}+00$ & $0.00 \mathrm{E}+00$ & $0.00 \mathrm{E}+00$ & $0.00 \mathrm{E}+00$ & $0.00 \mathrm{E}+00$ \\
\hline 241-B-201 & Solid & $1.55 \mathrm{E}+00$ & $2.82 \mathrm{E}-02$ & $4.33 \mathrm{E}-02$ & $7.51 \mathrm{E}-01$ & $6.09 \mathrm{E}-03$ & $1.24 \mathrm{E}-01$ \\
\hline $241-\mathrm{B}-202$ & Supernatant & $0.00 \mathrm{E}+00$ & $0.00 \mathrm{E}+00$ & $0.00 \mathrm{E}+00$ & $0.00 \mathrm{E}+00$ & $0.00 \mathrm{E}+00$ & $0.00 \mathrm{E}+00$ \\
\hline 241-B-202 & Solid & $2.72 \mathrm{E}+00$ & $6.67 \mathrm{E}-02$ & $2.16 \mathrm{E}-02$ & $1.28 \mathrm{E}-01$ & $1.97 \mathrm{E}-03$ & $6.96 \mathrm{E}-02$ \\
\hline $241-\mathrm{B}-203$ & Supernatant & $7.05 \mathrm{E}-05$ & $4.58 \mathrm{E}-05$ & $4.86 \mathrm{E}-04$ & $3.83 \mathrm{E}-03$ & $3.21 \mathrm{E}-05$ & $8.19 \mathrm{E}-05$ \\
\hline 241-B-203 & Solid & $6.30 \mathrm{E}-02$ & $3.46 \mathrm{E}-02$ & $2.95 \mathrm{E}-02$ & $2.33 \mathrm{E}-01$ & $1.96 \mathrm{E}-03$ & $6.03 \mathrm{E}-03$ \\
\hline 241-B-204 & Super & $3.14 \mathrm{E}-05$ & $1.78 \mathrm{E}-07$ & $4.70 \mathrm{E}-06$ & $6.98 \mathrm{E}-05$ & $2.29 \mathrm{E}-07$ & $3.62 \mathrm{E}-05$ \\
\hline 241-B-204 & Solid & $2.37 \mathrm{E}-03$ & $3.85 \mathrm{E}-02$ & $2.51 \mathrm{E}-02$ & $1.99 \mathrm{E}-01$ & $1.67 \mathrm{E}-03$ & $2.39 \mathrm{E}-02$ \\
\hline 241-BX-101 & Supernatant & $0.00 \mathrm{E}+00$ & $0.00 \mathrm{E}+00$ & $0.00 \mathrm{E}+00$ & $0.00 \mathrm{E}+00$ & $0.00 \mathrm{E}+00$ & $0.00 \mathrm{E}+00$ \\
\hline 241-BX-101 & Solid & $2.32 \mathrm{E}+02$ & $1.42 \mathrm{E}-01$ & $1.32 \mathrm{E}-01$ & $6.39 \mathrm{E}-01$ & $2.04 \mathrm{E}-02$ & $8.28 \mathrm{E}+01$ \\
\hline 241-BX-102 & Supernatant & $0.00 \mathrm{E}+00$ & $0.00 \mathrm{E}+00$ & $0.00 \mathrm{E}+00$ & $0.00 \mathrm{E}+00$ & $0.00 \mathrm{E}+00$ & $0.00 \mathrm{E}+00$ \\
\hline 241-BX-102 & Solid & $2.85 \mathrm{E}+02$ & $3.57 \mathrm{E}-03$ & $3.51 \mathrm{E}-03$ & $1.67 \mathrm{E}-02$ & $3.25 \mathrm{E}-04$ & $3.09 \mathrm{E}+01$ \\
\hline 241-BX-103 & Supernatant & $4.43 \mathrm{E}-01$ & $5.44 \mathrm{E}-03$ & $1.16 \mathrm{E}-03$ & $4.92 \mathrm{E}-03$ & $1.12 \mathrm{E}-04$ & $5.23 \mathrm{E}-01$ \\
\hline 241-BX-103 & Solid & $4.23 \mathrm{E}+02$ & $3.44 \mathrm{E}-01$ & $4.94 \mathrm{E}-01$ & $2.18 \mathrm{E}+00$ & $4.72 \mathrm{E}-02$ & $4.59 \mathrm{E}+01$ \\
\hline 241-BX-104 & Supernatant & $1.20 \mathrm{E}+00$ & $2.10 \mathrm{E}-04$ & $3.19 \mathrm{E}-05$ & $1.68 \mathrm{E}-04$ & $2.53 \mathrm{E}-06$ & $8.81 E+01$ \\
\hline 241-BX-104 & & $1.76 \mathrm{E}+02$ & $5.35 \mathrm{E}-01$ & $4.50 \mathrm{E}-02$ & $3.49 \mathrm{E}-01$ & $3.35 \mathrm{E}-03$ & $5.53 \mathrm{E}+01$ \\
\hline 241-BX-105 & Super & $5.30 \mathrm{E}+00$ & $1.91 \mathrm{E}-02$ & $2.53 \mathrm{E}-03$ & $1.08 \mathrm{E}-02$ & $2.46 \mathrm{E}-04$ & $8.44 \mathrm{E}+01$ \\
\hline $241-\mathrm{E}$ & Solid & $1.66 \mathrm{E}+02$ & $5.93 \mathrm{E}-02$ & $1.92 \mathrm{E}-02$ & $9.64 \mathrm{E}-02$ & $1.99 \mathrm{E}-03$ & $6.69 \mathrm{E}+01$ \\
\hline 241-BX-106 & Supernatant & $0.00 \mathrm{E}+00$ & $0.00 \mathrm{E}+00$ & $0.00 \mathrm{E}+00$ & $0.00 \mathrm{E}+00$ & $0.00 \mathrm{E}+00$ & $0.00 \mathrm{E}+00$ \\
\hline $241-$ & & $2.84 \mathrm{E}+02$ & $2.50 \mathrm{E}-01$ & $4.01 \mathrm{E}-02$ & $1.96 \mathrm{E}-01$ & $5.01 \mathrm{E}-03$ & $7.28 \mathrm{E}+01$ \\
\hline 241-BX-107 & Supernatant & $0.00 \mathrm{E}+00$ & $0.00 \mathrm{E}+00$ & $0.00 \mathrm{E}+00$ & $0.00 \mathrm{E}+00$ & $0.00 \mathrm{E}+00$ & $0.00 \mathrm{E}+00$ \\
\hline 241-BX-107 & Solid & $6.82 \mathrm{E}+00$ & $1.30 \mathrm{E}-02$ & $5.61 \mathrm{E}-03$ & $5.16 \mathrm{E}-02$ & $3.51 \mathrm{E}-04$ & $1.22 \mathrm{E}+01$ \\
\hline 241-BX-108 & Supernatant & $0.00 \mathrm{E}+00$ & $0.00 \mathrm{E}+00$ & $0.00 \mathrm{E}+00$ & $0.00 \mathrm{E}+00$ & $0.00 \mathrm{E}+00$ & $0.00 \mathrm{E}+00$ \\
\hline 241-BX-108 & Solid & $1.17 \mathrm{E}+02$ & $1.86 \mathrm{E}-02$ & $5.13 \mathrm{E}-03$ & $4.76 \mathrm{E}-02$ & $3.23 \mathrm{E}-04$ & $2.06 \mathrm{E}+01$ \\
\hline 241-BX-109 & Supernatant & $0.00 \mathrm{E}+00$ & $0.00 \mathrm{E}+00$ & $0.00 \mathrm{E}+00$ & $0.00 \mathrm{E}+00$ & $0.00 \mathrm{E}+00$ & $0.00 \mathrm{E}+00$ \\
\hline 241-BX-109 & & $9.73 E+01$ & $5.34 \mathrm{E}-03$ & $1.05 \mathrm{E}-03$ & $9.73 \mathrm{E}-03$ & $6.57 \mathrm{E}-05$ & $9.82 \mathrm{E}+00$ \\
\hline 241-BX-110 & Supernatant & $1.21 \mathrm{E}+00$ & $3.92 \mathrm{E}-03$ & $7.00 \mathrm{E}-04$ & $2.92 \mathrm{E}-03$ & $1.09 \mathrm{E}-04$ & $8.07 \mathrm{E}+01$ \\
\hline $241-$ & Solid & $7.14 \mathrm{E}+00$ & $4.73 \mathrm{E}-03$ & $1.81 \mathrm{E}-03$ & $1.52 \mathrm{E}-02$ & $1.40 \mathrm{E}-04$ & $4.47 \mathrm{E}+01$ \\
\hline 241-BX-111 & upernatant & $0.00 \mathrm{E}+00$ & $0.00 \mathrm{E}+00$ & $0.00 \mathrm{E}+00$ & $0.00 \mathrm{E}+00$ & $0.00 \mathrm{E}+00$ & $0.00 \mathrm{E}+00$ \\
\hline $\mathrm{X}-111$ & & $1.05 \mathrm{E}+01$ & $3.43 \mathrm{E}-03$ & $1.64 \mathrm{E}-03$ & $1.45 \mathrm{E}-02$ & $1.14 \mathrm{E}-04$ & $5.90 \mathrm{E}+01$ \\
\hline $\mathrm{X}-112$ & Supernatant & $5.64 \mathrm{E}-01$ & $1.38 \mathrm{E}-04$ & $1.92 \mathrm{E}-04$ & $1.35 \mathrm{E}-03$ & $1.30 \mathrm{E}-05$ & $6.66 \mathrm{E}-01$ \\
\hline 241-BX-112 & Solid & $5.28 \mathrm{E}+00$ & $2.41 \mathrm{E}-02$ & $1.58 \mathrm{E}-02$ & $1.45 \mathrm{E}-01$ & $9.91 \mathrm{E}-04$ & $3.70 \mathrm{E}+01$ \\
\hline 241-BY-101 & Supernatant & $0.00 \mathrm{E}+00$ & $0.00 \mathrm{E}+00$ & $0.00 \mathrm{E}+00$ & $0.00 \mathrm{E}+00$ & $0.00 \mathrm{E}+00$ & $0.00 \mathrm{E}+00$ \\
\hline 241-BY-101 & Solid & $5.22 \mathrm{E}+01$ & $9.93 \mathrm{E}-03$ & $1.24 \mathrm{E}-03$ & $1.08 \mathrm{E}-02$ & $9.13 \mathrm{E}-05$ & $8.28 \mathrm{E}+01$ \\
\hline 241-BY-102 & Supernatant & $0.00 \mathrm{E}+00$ & $0.00 \mathrm{E}+00$ & $0.00 \mathrm{E}+00$ & $0.00 \mathrm{E}+00$ & $0.00 \mathrm{E}+00$ & $0.00 \mathrm{E}+00$ \\
\hline 241-BY-102 & Solid & $9.54 \mathrm{E}+00$ & $5.04 \mathrm{E}-02$ & $5.15 \mathrm{E}-03$ & $2.16 \mathrm{E}-02$ & $8.02 \mathrm{E}-04$ & $6.06 \mathrm{E}+01$ \\
\hline 241-BY-103 & Supernatant & $0.00 \mathrm{E}+00$ & $0.00 \mathrm{E}+00$ & $0.00 \mathrm{E}+00$ & $0.00 \mathrm{E}+00$ & $0.00 \mathrm{E}+00$ & $0.00 \mathrm{E}+00$ \\
\hline 241-BY-103 & Solid & $1.03 \mathrm{E}+01$ & $1.16 \mathrm{E}-02$ & $1.34 \mathrm{E}-03$ & $5.61 \mathrm{E}-03$ & $1.99 \mathrm{E}-04$ & $6.08 \mathrm{E}+01$ \\
\hline 241-BY-104 & Supernatant & $0.00 \mathrm{E}+00$ & $0.00 \mathrm{E}+00$ & $0.00 \mathrm{E}+00$ & $0.00 \mathrm{E}+00$ & $0.00 \mathrm{E}+00$ & $0.00 \mathrm{E}+00$ \\
\hline 241-BY-104 & Solid & $1.25 \mathrm{E}+02$ & $5.52 \mathrm{E}-02$ & $5.83 \mathrm{E}-03$ & $2.94 \mathrm{E}-02$ & $8.21 \mathrm{E}-04$ & $7.91 \mathrm{E}+01$ \\
\hline
\end{tabular}


Table A-2. Tank Waste Radionuclide Concentrations.

\begin{tabular}{|c|c|c|c|c|c|c|c|}
\hline Tank & Layer & $\begin{array}{c}{ }^{90} \mathrm{Sr} \\
(\mu \mathrm{Ci} / \mathrm{g})\end{array}$ & $\begin{array}{l}{ }^{241} \mathrm{Am} \\
(\mu \mathrm{Ci} / \mathrm{g})\end{array}$ & $\begin{array}{c}{ }^{240} \mathrm{Pu} \\
(\mu \mathrm{Ci} / \mathrm{g})\end{array}$ & $\begin{array}{c}{ }^{239} \mathrm{Pu} \\
(\mu \mathrm{Ci} / \mathrm{g})\end{array}$ & $\begin{array}{c}{ }^{238} \mathrm{Pu} \\
(\mu \mathrm{Ci} / \mathrm{g})\end{array}$ & $\begin{array}{c}{ }^{137} \mathrm{Cs} \\
(\mu \mathrm{Ci} / \mathrm{g})\end{array}$ \\
\hline 241-BY-105 & Supernatant & $0.00 \mathrm{E}+00$ & $0.00 \mathrm{E}+00$ & $0.00 \mathrm{E}+00$ & $0.00 \mathrm{E}+00$ & $0.00 \mathrm{E}+00$ & $0.00 \mathrm{E}+00$ \\
\hline 241-BY-105 & Solid & $3.89 \mathrm{E}+01$ & $3.60 \mathrm{E}-02$ & $3.91 \mathrm{E}-03$ & $2.38 \mathrm{E}-02$ & $4.73 \mathrm{E}-04$ & $4.24 \mathrm{E}+01$ \\
\hline 241-BY-106 & Supernatant & $0.00 \mathrm{E}+00$ & $0.00 \mathrm{E}+00$ & $0.00 \mathrm{E}+00$ & $0.00 \mathrm{E}+00$ & $0.00 \mathrm{E}+00$ & $0.00 \mathrm{E}+00$ \\
\hline 241-BY-106 & Solid & $4.62 \mathrm{E}+01$ & $1.44 \mathrm{E}-02$ & $1.20 \mathrm{E}-03$ & $8.72 \mathrm{E}-03$ & $1.19 \mathrm{E}-04$ & $1.03 E+02$ \\
\hline 241-BY-107 & Supernatant & $0.00 \mathrm{E}+00$ & $0.00 \mathrm{E}+00$ & $0.00 \mathrm{E}+00$ & $0.00 \mathrm{E}+00$ & $0.00 \mathrm{E}+00$ & $0.00 \mathrm{E}+00$ \\
\hline 241-BY-107 & Solid & $3.90 \mathrm{E}+01$ & $1.18 \mathrm{E}-02$ & $1.33 \mathrm{E}-03$ & $8.10 \mathrm{E}-03$ & $1.61 \mathrm{E}-04$ & $1.04 \mathrm{E}+02$ \\
\hline 241-BY-108 & Supernatant & $0.00 \mathrm{E}+00$ & $0.00 \mathrm{E}+00$ & $0.00 \mathrm{E}+00$ & $0.00 \mathrm{E}+00$ & $0.00 \mathrm{E}+00$ & $0.00 \mathrm{E}+00$ \\
\hline 241-BY-108 & Solid & $9.38 \mathrm{E}+01$ & $3.04 \mathrm{E}-02$ & $3.43 \mathrm{E}-03$ & $2.26 \mathrm{E}-02$ & $3.83 \mathrm{E}-04$ & $3.84 \mathrm{E}+01$ \\
\hline 241-BY-109 & Supernatant & $0.00 \mathrm{E}+00$ & $0.00 \mathrm{E}+00$ & $0.00 \mathrm{E}+00$ & $0.00 \mathrm{E}+00$ & $0.00 \mathrm{E}+00$ & $0.00 \mathrm{E}+00$ \\
\hline 241-BY-109 & Solid & $7.04 \mathrm{E}+00$ & $3.69 \mathrm{E}-02$ & $4.35 \mathrm{E}-03$ & $1.82 \mathrm{E}-02$ & $6.42 \mathrm{E}-04$ & $4.54 \mathrm{E}+01$ \\
\hline 241-BY-110 & Supernatant & $0.00 \mathrm{E}+00$ & $0.00 \mathrm{E}+00$ & $0.00 \mathrm{E}+00$ & $0.00 \mathrm{E}+00$ & $0.00 \mathrm{E}+00$ & $0.00 \mathrm{E}+00$ \\
\hline $241-\mathrm{BY}-110$ & Solid & $6.61 \mathrm{E}+01$ & $2.75 \mathrm{E}-02$ & $3.81 \mathrm{E}-03$ & $2.22 \mathrm{E}-02$ & $4.81 \mathrm{E}-04$ & $7.98 \mathrm{E}+01$ \\
\hline 241-BY-111 & Supernatant & $0.00 \mathrm{E}+00$ & $0.00 \mathrm{E}+00$ & $0.00 \mathrm{E}+00$ & $0.00 \mathrm{E}+00$ & $0.00 \mathrm{E}+00$ & $0.00 \mathrm{E}+00$ \\
\hline 241-BY-111 & Solid & $9.32 \mathrm{E}+00$ & $6.14 \mathrm{E}-02$ & $6.28 \mathrm{E}-03$ & $2.63 \mathrm{E}-02$ & $9.79 \mathrm{E}-04$ & $5.43 \mathrm{E}+01$ \\
\hline 241-BY-112 & Supernatant & $0.00 \mathrm{E}+00$ & $0.00 \mathrm{E}+00$ & $0.00 \mathrm{E}+00$ & $0.00 \mathrm{E}+00$ & $0.00 \mathrm{E}+00$ & $0.00 \mathrm{E}+00$ \\
\hline 241-BY-112 & Solid & $1.07 \mathrm{E}+01$ & $4.29 \mathrm{E}-02$ & $4.40 \mathrm{E}-03$ & $1.84 \mathrm{E}-02$ & $6.87 \mathrm{E}-04$ & $5.80 \mathrm{E}+01$ \\
\hline $241-\mathrm{C}-101$ & Super & $0.00 \mathrm{E}+00$ & $0.00 \mathrm{E}+00$ & $0.00 \mathrm{E}+00$ & $0.00 \mathrm{E}+00$ & $0.00 \mathrm{E}+00$ & $0.00 \mathrm{E}+00$ \\
\hline 241 & & $5.82 \mathrm{E}+01$ & $1.45 \mathrm{~B}$ & $1.39 \mathrm{E}-01$ & $7.86 \mathrm{E}-01$ & $1.07 \mathrm{E}-02$ & $4.63 \mathrm{E}+01$ \\
\hline 241 & Supe & $0.00 \mathrm{E}+00$ & $0.00 \mathrm{E}+00$ & $0.00 \mathrm{E}+00$ & $0.00 \mathrm{E}+00$ & $0.00 \mathrm{E}+00$ & $0.00 \mathrm{E}+00$ \\
\hline 102 & $\mathrm{~S}$ & $1.27 \mathrm{E}+01$ & $5.21 \mathrm{E}-01$ & $4.05 \mathrm{E}-01$ & $1.69 \mathrm{E}+00$ & $4.24 \mathrm{E}-02$ & $1.17 \mathrm{E}+01$ \\
\hline $241-C$ & Supernatant & $6.63 \mathrm{E}-02$ & $5.09 \mathrm{E}-06$ & $1.60 \mathrm{E}-06$ & $1.03 \mathrm{E}-05$ & $2.81 \mathrm{E}-06$ & $9.80 \mathrm{E}-02$ \\
\hline $241-C$ & & $6.51 \mathrm{E}+02$ & $3.54 \mathrm{E}-01$ & $7.48 \mathrm{E}-02$ & $3.59 \mathrm{E}-01$ & $1.03 \mathrm{E}-01$ & $5.75 \mathrm{E}+01$ \\
\hline $241-C-104$ & Supernatant & $0.00 \mathrm{E}+00$ & $0.00 \mathrm{E}+00$ & $0.00 \mathrm{E}+00$ & $0.00 \mathrm{E}+00$ & $0.00 \mathrm{E}+00$ & $0.00 \mathrm{E}+00$ \\
\hline $241-C-104$ & Solid & $2.47 \mathrm{E}+02$ & $2.72 \mathrm{E}+00$ & $4.92 \mathrm{E}-01$ & $1.91 \mathrm{E}+00$ & $2.65 \mathrm{E}-01$ & $4.91 \mathrm{E}+01$ \\
\hline & Supernatant & $0.00 \mathrm{E}+00$ & $0.00 \mathrm{E}+00$ & $0.00 \mathrm{E}+00$ & $0.00 \mathrm{E}+00$ & $0.00 \mathrm{E}+00$ & $0.00 \mathrm{E}+00$ \\
\hline$\overline{241}$ & & $5.18 \mathrm{E}+02$ & $3.89 \mathrm{E}-01$ & $1.40 \mathrm{E}-01$ & $7.07 \mathrm{E}-01$ & $1.11 \mathrm{E}-02$ & $8.99 \mathrm{E}+01$ \\
\hline & & $3.93 \mathrm{E}-02$ & 4.14 & $8.49 \mathrm{E}-07$ & $3.99 \mathrm{E}$ & $6.21 \mathrm{E}-07$ & $3.90 \mathrm{E}-01$ \\
\hline 241 & & $3.79 \mathrm{E}+03$ & $4.10 \mathrm{I}$ & $2.25 \mathrm{E}-01$ & $1.05 \mathrm{E}+00$ & $1.65 \mathrm{E}-01$ & $8.32 \mathrm{E}+01$ \\
\hline 241 & Sup & $0.00 \mathrm{E}+00$ & $0.00 \mathrm{E}+00$ & $0.00 \mathrm{E}+00$ & $0.00 \mathrm{E}+00$ & $0.00 \mathrm{E}+00$ & $0.00 \mathrm{E}+00$ \\
\hline 241 & & $1.31 \mathrm{E}+03$ & $4.59 \mathrm{E}+00$ & $2.44 \mathrm{E}-01$ & $1.51 \mathrm{E}+00$ & $2.96 \mathrm{E}-02$ & $3.71 \mathrm{E}+01$ \\
\hline 241 & natant & $8.32 \mathrm{E}-01$ & $1.88 \mathrm{E}-04$ & $2.97 \mathrm{E}-04$ & $1.41 \mathrm{E}-03$ & $2.00 \mathrm{E}-04$ & $1.70 \mathrm{E}+01$ \\
\hline $241-C-108$ & Solid & $1.96 \mathrm{E}+01$ & $2.90 \mathrm{E}-02$ & $9.18 \mathrm{E}-04$ & $8.44 \mathrm{E}-03$ & $5.74 \mathrm{E}-05$ & $1.91 \mathrm{E}+02$ \\
\hline $241-C-109$ & Supernatant & $0.00 \mathrm{E}+00$ & $0.00 \mathrm{E}+00$ & $0.00 \mathrm{E}+00$ & $0.00 \mathrm{E}+00$ & $0.00 \mathrm{E}+00$ & $0.00 \mathrm{E}+00$ \\
\hline 241-C-109 & Solid & $4.38 \mathrm{E}+00$ & $1.38 \mathrm{E}-02$ & 9.07E-03 & $8.32 \mathrm{E}-02$ & $5.69 \mathrm{E}-04$ & $1.45 \mathrm{E}+01$ \\
\hline & Supernatant & $0.00 \mathrm{E}+00$ & $0.00 \mathrm{E}+00$ & $0.00 \mathrm{E}+00$ & $0.00 \mathrm{E}+00$ & $0.00 \mathrm{E}+00$ & $0.00 \mathrm{E}+00$ \\
\hline & & $3.49 \mathrm{E}+00$ & $4.27 \mathrm{E}-02$ & $7.88 \mathrm{E}-03$ & $7.23 \mathrm{E}-02$ & $4.92 \mathrm{E}-04$ & $1.37 \mathrm{E}+01$ \\
\hline $241-C$ & Supern & $0.00 \mathrm{E}+00$ & $0.00 \mathrm{E}-$ & $0.00 \mathrm{E}+00$ & $0.00 \mathrm{E}+00$ & $0.00 \mathrm{E}+00$ & $0.00 \mathrm{E}+00$ \\
\hline $241-C$ & & $2.38 \mathrm{E}+03$ & $5.50 \mathrm{E}-01$ & $9.56 \mathrm{E}-02$ & $4.92 \mathrm{E}-01$ & $8.75 \mathrm{E}-03$ & $5.19 \mathrm{E}+01$ \\
\hline 241 & atant & $0.00 \mathrm{E}+00$ & $0.00 \mathrm{E}+00$ & $0.00 \mathrm{E}+00$ & $0.00 \mathrm{E}+00$ & $0.00 \mathrm{E}+00$ & $0.00 \mathrm{E}+00$ \\
\hline $241-\mathrm{C}$ & & $7.55 \mathrm{E}+02$ & $6.49 \mathrm{E}-01$ & $1.62 \mathrm{E}-02$ & $1.27 \mathrm{E}-01$ & $1.06 \mathrm{E}-02$ & $3.37 \mathrm{E}+02$ \\
\hline 241-C-201 & upernatant & $0.00 \mathrm{E}+00$ & $0.00 \mathrm{E}+00$ & $0.00 \mathrm{E}+00$ & $0.00 \mathrm{E}+00$ & $0.00 \mathrm{E}+00$ & $0.00 \mathrm{E}+00$ \\
\hline $241-C-201$ & Solid & $2.43 \mathrm{E}+02$ & $2.67 \mathrm{E}+00$ & $3.62 \mathrm{E}+00$ & $1.68 \mathrm{E}+01$ & $5.17 \mathrm{E}-01$ & $9.83 E+00$ \\
\hline $241-C-202$ & Supernatant & $0.00 \mathrm{E}+00$ & $0.00 \mathrm{E}+00$ & $0.00 \mathrm{E}+00$ & $0.00 \mathrm{E}+00$ & $0.00 \mathrm{E}+00$ & $0.00 \mathrm{E}+00$ \\
\hline $241-\mathrm{C}-202$ & Solid & $4.61 \mathrm{E}+02$ & $1.28 \mathrm{E}+00$ & $3.21 \mathrm{E}+00$ & $1.49 \mathrm{E}+01$ & $4.58 \mathrm{E}-01$ & $8.50 \mathrm{E}+00$ \\
\hline $241-\mathrm{C}-203$ & Supernatant & $0.00 \mathrm{E}+00$ & $0.00 \mathrm{E}+00$ & $0.00 \mathrm{E}+00$ & $0.00 \mathrm{E}+00$ & $0.00 \mathrm{E}+00$ & $0.00 \mathrm{E}+00$ \\
\hline $241-C-203$ & Solid & $2.26 \mathrm{E}+02$ & $3.51 \mathrm{E}-02$ & $1.14 \mathrm{E}-01$ & $5.29 \mathrm{E}-01$ & $1.63 \mathrm{E}-02$ & $1.31 \mathrm{E}+01$ \\
\hline $241-C-204$ & Supernatant & $0.00 \mathrm{E}+00$ & $0.00 \mathrm{E}+00$ & $0.00 \mathrm{E}+00$ & $0.00 \mathrm{E}+00$ & $0.00 \mathrm{E}+00$ & $0.00 \mathrm{E}+00$ \\
\hline 241-C-204 & Solid & $1.54 \mathrm{E}+02$ & $3.59 \mathrm{E}-03$ & $2.36 \mathrm{E}-03$ & $1.10 \mathrm{E}-02$ & $3.38 \mathrm{E}-04$ & $6.06 \mathrm{E}+00$ \\
\hline 241-S-101 & Supernatant & $0.00 \mathrm{E}+00$ & $0.00 \mathrm{E}+00$ & $0.00 \mathrm{E}+00$ & $0.00 \mathrm{E}+00$ & $0.00 \mathrm{E}+00$ & $0.00 \mathrm{E}+00$ \\
\hline 241-S-101 & Solid & $1.87 \mathrm{E}+02$ & $1.00 \mathrm{E}-01$ & $2.87 \mathrm{E}-02$ & $1.46 \mathrm{E}-01$ & $2.95 \mathrm{E}-03$ & $1.02 \mathrm{E}+02$ \\
\hline
\end{tabular}


Table A-2. Tank Waste Radionuclide Concentrations.

\begin{tabular}{|c|c|c|c|c|c|c|c|}
\hline Tank & Layer & $\begin{array}{c}{ }^{90} \mathrm{Sr} \\
(\mu \mathrm{Ci} / \mathrm{g})\end{array}$ & $\begin{array}{l}{ }^{241} \mathrm{Am} \\
(\mu \mathrm{Ci} / \mathrm{g})\end{array}$ & $\begin{array}{l}{ }^{240} \mathrm{Pu} \\
(\mu \mathrm{Ci} / \mathrm{g})\end{array}$ & $\begin{array}{c}{ }^{239} \mathrm{Pu} \\
(\mu \mathrm{Ci} / \mathrm{g})\end{array}$ & $\begin{array}{c}{ }^{238} \mathrm{Pu} \\
(\mu \mathrm{Ci} / \mathrm{g})\end{array}$ & $\begin{array}{c}{ }^{137} \mathrm{Cs} \\
(\mu \mathrm{Ci} / \mathrm{g})\end{array}$ \\
\hline $241-S-102$ & Supernatant & $0.00 \mathrm{E}+00$ & $0.00 \mathrm{E}+00$ & $0.00 \mathrm{E}+00$ & $0.00 \mathrm{E}+00$ & $0.00 \mathrm{E}+00$ & $0.00 \mathrm{E}+00$ \\
\hline $241-\mathrm{S}-102$ & Solid & $2.28 \mathrm{E}+02$ & $6.27 \mathrm{E}-02$ & $1.20 \mathrm{E}-02$ & $5.90 \mathrm{E}-02$ & $1.43 \mathrm{E}-03$ & $7.16 \mathrm{E}+01$ \\
\hline $241-S-103$ & Supernatant & $2.31 \mathrm{E}-01$ & $8.09 \mathrm{E}-04$ & $8.45 \mathrm{E}-06$ & $3.93 \mathrm{E}-05$ & $1.33 \mathrm{E}-06$ & $2.03 E+02$ \\
\hline $241-S-103$ & Solid & $3.33 \mathrm{E}+01$ & $1.19 \mathrm{E}-01$ & $1.73 \mathrm{E}-02$ & $8.30 \mathrm{E}-02$ & $2.39 \mathrm{E}-03$ & $1.56 \mathrm{E}+02$ \\
\hline $241-S-104$ & Supernatant & $0.00 \mathrm{E}+00$ & $0.00 \mathrm{E}+00$ & $0.00 \mathrm{E}+00$ & $0.00 \mathrm{E}+00$ & $0.00 \mathrm{E}+00$ & $0.00 \mathrm{E}+00$ \\
\hline $241-S-104$ & Solid & $1.94 \mathrm{E}+02$ & $1.07 \mathrm{E}-01$ & $3.83 \mathrm{E}-02$ & $1.88 \mathrm{E}-01$ & $5.21 \mathrm{E}-03$ & $3.90 \mathrm{E}+01$ \\
\hline $241-\mathrm{S}-1$ & Supe & $0.00 \mathrm{E}+00$ & $0.00 \mathrm{E}-$ & $0.00 \mathrm{E}+00$ & $0.00 \mathrm{E}+00$ & $0.00 \mathrm{E}+00$ & $0.00 \mathrm{E}+00$ \\
\hline $241-S-105$ & & $2.32 \mathrm{E}+00$ & $3.05 \mathrm{E}-03$ & $7.25 \mathrm{E}-04$ & $3.63 \mathrm{E}-03$ & $7.94 \mathrm{E}-05$ & $3.12 \mathrm{E}+01$ \\
\hline $241-S-106$ & Supernatant & $0.00 \mathrm{E}+00$ & $0.00 \mathrm{E}+00$ & $0.00 \mathrm{E}+00$ & $0.00 \mathrm{E}+00$ & $0.00 \mathrm{E}+00$ & $0.00 \mathrm{E}+00$ \\
\hline $241-S-106$ & Solid & $1.11 \mathrm{E}+01$ & $1.96 \mathrm{E}-02$ & $1.32 \mathrm{E}-03$ & $6.04 \mathrm{E}-03$ & $2.06 \mathrm{E}-04$ & $8.32 \mathrm{E}+01$ \\
\hline 241-S-107 & Supernatant & $0.00 \mathrm{E}+00$ & $0.00 \mathrm{E}+00$ & $0.00 \mathrm{E}+00$ & $0.00 \mathrm{E}+00$ & $0.00 \mathrm{E}+00$ & $0.00 \mathrm{E}+00$ \\
\hline $241-S-107$ & Solid & $1.18 \mathrm{E}+02$ & $3.11 \mathrm{E}-01$ & $1.06 \mathrm{E}-01$ & $5.04 \mathrm{E}-01$ & $1.18 \mathrm{E}-02$ & $6.18 \mathrm{E}+01$ \\
\hline $241-S-108$ & Supernatant & $0.00 \mathrm{E}+00$ & $0.00 \mathrm{E}+00$ & $0.00 \mathrm{E}+00$ & $0.00 \mathrm{E}+00$ & $0.00 \mathrm{E}+00$ & $0.00 \mathrm{E}+00$ \\
\hline $241-S-108$ & Solid & $1.90 \mathrm{E}+01$ & $7.69 \mathrm{E}-02$ & $5.96 \mathrm{E}-03$ & $2.79 \mathrm{E}-02$ & $8.76 \mathrm{E}-04$ & $8.73 E+01$ \\
\hline $241-S-109$ & $\tan t$ & $0.00 \mathrm{E}+00$ & $0.00 \mathrm{E}+00$ & $0.00 \mathrm{E}+00$ & $0.00 \mathrm{E}+00$ & $0.00 \mathrm{E}+00$ & $0.00 \mathrm{E}+00$ \\
\hline 241 & & $1.35 \mathrm{E}+01$ & $1.14 \mathrm{E}-02$ & $2.83 \mathrm{E}-03$ & $1.44 \mathrm{E}-02$ & $2.83 \mathrm{E}-04$ & $1.19 \mathrm{E}+01$ \\
\hline 241 & $\tan t$ & $0.00 \mathrm{E}+$ & $0.00 \mathrm{E}$ & $0.00 \mathrm{E}+00$ & $0.00 \mathrm{E}$ & $0.00 \mathrm{E}$ & $0.00 \mathrm{E}+00$ \\
\hline $241-5$ & & $8.53 \mathrm{E}+01$ & $1.23 \mathrm{E}-01$ & $2.49 \mathrm{E}-02$ & $1.26 \mathrm{E}-01$ & $2.55 \mathrm{E}-03$ & $6.63 \mathrm{E}+01$ \\
\hline $241-$ & $\tan t$ & $0.00 \mathrm{E}+00$ & $0.00 \mathrm{E}+00$ & $0.00 \mathrm{E}+00$ & $0.00 \mathrm{E}+00$ & $0.00 \mathrm{E}+00$ & $0.00 \mathrm{E}+00$ \\
\hline $241-\mathrm{S}-111$ & Solid & $1.66 \mathrm{E}+02$ & $1.48 \mathrm{E}-02$ & $1.43 \mathrm{E}-03$ & $6.89 \mathrm{E}-03$ & $1.90 \mathrm{E}-04$ & $9.37 \mathrm{E}+01$ \\
\hline $241-S-112$ & Supernatant & $0.00 \mathrm{E}+00$ & $0.00 \mathrm{E}+00$ & $0.00 \mathrm{E}+00$ & $0.00 \mathrm{E}+00$ & $0.00 \mathrm{E}+00$ & $0.00 \mathrm{E}+00$ \\
\hline 241-S-112 & Solid & $3.01 \mathrm{E}+00$ & $7.80 \mathrm{E}-04$ & $2.68 \mathrm{E}-04$ & $1.38 \mathrm{E}-03$ & $2.48 \mathrm{E}-05$ & $1.80 \mathrm{E}-01$ \\
\hline $241-\mathrm{S} \lambda$ & Supernatant & $0.00 \mathrm{E}+00$ & $0.00 \mathrm{E}+00$ & $0.00 \mathrm{E}+00$ & $0.00 \mathrm{E}+00$ & $0.00 \mathrm{E}+00$ & $0.00 \mathrm{E}+00$ \\
\hline $\mathrm{X}-101$ & & $1.03 \mathrm{E}+02$ & $1.91 \mathrm{E}-01$ & $3.39 \mathrm{E}-02$ & $1.66 \mathrm{E}-01$ & $4.45 \mathrm{E}-03$ & $9.16 \mathrm{E}+01$ \\
\hline & 5 & $0.00 \mathrm{E}+00$ & $0.00 \mathrm{E}-$ & $0.00 \mathrm{E}+00$ & $0.00 \mathrm{E}+$ & $0.00 \mathrm{E}$ & $0.00 \mathrm{E}+00$ \\
\hline 241 & & 01 & 1.22 & 1.211 & $5.77 \mathrm{E}$ & $1.64 \mathrm{~F}$ & $1.28 \mathrm{E}+02$ \\
\hline $241-$ & it & 00 & $0.00 \mathrm{E}$ & $0.00 \mathrm{E}$ & $0.00 \mathrm{E}$ & +00 & $0.00 \mathrm{E}+00$ \\
\hline 241-SX-103 & & $1.40 \mathrm{E}+02$ & $2.15 \mathrm{E}-01$ & $1.56 \mathrm{E}-02$ & $7.61 \mathrm{E}-02$ & $1.98 \mathrm{E}-03$ & $1.13 \mathrm{E}+02$ \\
\hline $241-$ & upernatant & $0.00 \mathrm{E}+00$ & $0.00 \mathrm{E}+00$ & $0.00 \mathrm{E}+00$ & $0.00 \mathrm{E}+00$ & $0.00 \mathrm{E}+00$ & $0.00 \mathrm{E}+00$ \\
\hline 241-SX-104 & Solid & $1.29 \mathrm{E}+02$ & $1.25 \mathrm{E}-01$ & $3.42 \mathrm{E}-02$ & $1.74 \mathrm{E}-01$ & $3.41 \mathrm{E}-03$ & $7.92 \mathrm{E}+01$ \\
\hline 241-SX-105 & Supernatant & $0.00 \mathrm{E}+00$ & $0.00 \mathrm{E}+00$ & $0.00 \mathrm{E}+00$ & $0.00 \mathrm{E}+00$ & $0.00 \mathrm{E}+00$ & $0.00 \mathrm{E}+00$ \\
\hline 241-SX-105 & Solid & $2.29 \mathrm{E}+02$ & $2.56 \mathrm{E}-01$ & $5.74 \mathrm{E}-02$ & $2.89 \mathrm{E}-01$ & $6.42 \mathrm{E}-03$ & $8.64 \mathrm{E}+01$ \\
\hline $241-\mathrm{SX}-106$ & Supernatant & $0.00 \mathrm{E}+00$ & $0.00 \mathrm{E}+00$ & $0.00 \mathrm{E}+00$ & $0.00 \mathrm{E}+00$ & $0.00 \mathrm{E}+00$ & $0.00 \mathrm{E}+00$ \\
\hline 241-SX-106 & lid & $1.10 \mathrm{E}+01$ & $1.47 \mathrm{E}-01$ & $1.86 \mathrm{E}-02$ & $8.68 \mathrm{E}-02$ & $2.93 \mathrm{E}-03$ & $1.20 \mathrm{E}+02$ \\
\hline & & & & $0.00 \mathrm{E}+00$ & 0.00 & $0.00 \mathrm{E}+00$ & $0.00 \mathrm{E}+00$ \\
\hline & & & 01 & 01 & -01 & -02 & $7.47 \mathrm{E}+01$ \\
\hline $241-5$ & Super & +00 & 0.001 & $0.00 \mathrm{E}+00$ & $0.00 \mathrm{~F}$ & $00 \mathrm{E}+00$ & $0.00 \mathrm{E}+00$ \\
\hline $241-$ & & $2.34 \mathrm{E}+03$ & $1.46 \mathrm{E}+00$ & $2.96 \mathrm{E}-01$ & $1.49 E+00$ & $3.66 \mathrm{E}-02$ & $1.50 \mathrm{E}+02$ \\
\hline $241-$ & Supernatant & $0.00 \mathrm{E}+00$ & $0.00 \mathrm{E}+00$ & $0.00 \mathrm{E}+00$ & $0.00 \mathrm{E}+00$ & $0.00 \mathrm{E}+00$ & $0.00 \mathrm{E}+00$ \\
\hline 241-SX-109 & Solid & $5.17 \mathrm{E}+02$ & $2.91 \mathrm{E}-01$ & $3.75 \mathrm{E}-02$ & $1.79 \mathrm{E}-01$ & $6.27 \mathrm{E}-03$ & $7.21 \mathrm{E}+01$ \\
\hline 241-SX-110 & Supernatant & $0.00 \mathrm{E}+00$ & $0.00 \mathrm{E}+00$ & $0.00 \mathrm{E}+00$ & $0.00 \mathrm{E}+00$ & $0.00 \mathrm{E}+00$ & $0.00 \mathrm{E}+00$ \\
\hline 241-SX-110 & Solid & $4.15 \mathrm{E}+03$ & $1.90 \mathrm{E}+00$ & $1.18 \mathrm{E}-01$ & $5.04 \mathrm{E}-01$ & $3.23 \mathrm{E}-02$ & $1.05 \mathrm{E}+02$ \\
\hline $241-\mathrm{SX}-111$ & Supernatant & $0.00 \mathrm{E}+00$ & $0.00 \mathrm{E}+00$ & $0.00 \mathrm{E}+00$ & $0.00 \mathrm{E}+00$ & $0.00 \mathrm{E}+00$ & $0.00 \mathrm{E}+00$ \\
\hline 241-SX-111 & Solid & $2.40 \mathrm{E}+03$ & $1.13 \mathrm{E}+00$ & $1.01 \mathrm{E}-01$ & $4.64 \mathrm{E}-01$ & $2.11 \mathrm{E}-02$ & $8.43 \mathrm{E}+01$ \\
\hline 241-SX-112 & Supernatant & $0.00 \mathrm{E}+00$ & $0.00 \mathrm{E}+00$ & $0.00 \mathrm{E}+00$ & $0.00 \mathrm{E}+00$ & $0.00 \mathrm{E}+00$ & $0.00 \mathrm{E}+00$ \\
\hline 241-SX-112 & Solid & $2.53 \mathrm{E}+03$ & $1.20 \mathrm{E}+00$ & $1.15 \mathrm{E}-01$ & $5.31 \mathrm{E}-01$ & $2.27 \mathrm{E}-02$ & $8.34 \mathrm{E}+01$ \\
\hline 241-SX-113 & Supernatant & $0.00 \mathrm{E}+00$ & $0.00 \mathrm{E}+00$ & $0.00 \mathrm{E}+00$ & $0.00 \mathrm{E}+00$ & $0.00 \mathrm{E}+00$ & $0.00 \mathrm{E}+00$ \\
\hline 241-SX-113 & & $2.50 \mathrm{E}+01$ & $1.08 \mathrm{E}-01$ & $9.47 \mathrm{E}-03$ & $4.88 \mathrm{E}-02$ & $8.77 \mathrm{E}-04$ & $1.84 \mathrm{E}+01$ \\
\hline & Supernatant & $0.00 \mathrm{E}+00$ & $0.00 \mathrm{E}+00$ & $0.00 \mathrm{E}+00$ & $0.00 \mathrm{E}+00$ & $0.00 \mathrm{E}+00$ & $0.00 \mathrm{E}+00$ \\
\hline $241-\mathrm{SX}-114$ & Solid & $1.67 \mathrm{E}+03$ & $8.12 \mathrm{E}-01$ & $9.20 \mathrm{E}-02$ & $4.37 \mathrm{E}-01$ & $1.63 \mathrm{E}-02$ & $7.99 \mathrm{E}+01$ \\
\hline
\end{tabular}


Table A-2. Tank Waste Radionuclide Concentrations.

\begin{tabular}{|c|c|c|c|c|c|c|c|}
\hline Tank & Layer & $\begin{array}{c}{ }^{90} \mathrm{Sr} \\
(\mu \mathrm{Ci} / \mathrm{g})\end{array}$ & $\begin{array}{l}{ }^{241} \mathrm{Am} \\
(\mu \mathrm{Ci} / \mathrm{g})\end{array}$ & $\begin{array}{c}{ }^{240} \mathrm{Pu} \\
(\mu \mathrm{Ci} / \mathrm{g})\end{array}$ & $\begin{array}{c}{ }^{239} \mathrm{Pu} \\
(\mu \mathrm{Ci} / \mathrm{g})\end{array}$ & $\begin{array}{c}{ }^{238} \mathrm{Pu} \\
(\mu \mathrm{Ci} / \mathrm{g})\end{array}$ & $\begin{array}{c}{ }^{137} \mathrm{Cs} \\
(\mu \mathrm{Ci} / \mathrm{g})\end{array}$ \\
\hline $241-\mathrm{SX}-115$ & Supernatant & $0.00 \mathrm{E}+00$ & $0.00 \mathrm{E}+00$ & $0.00 \mathrm{E}+00$ & $0.00 \mathrm{E}+00$ & $0.00 \mathrm{E}+00$ & $0.00 \mathrm{E}+00$ \\
\hline $241-\mathrm{SX}-115$ & Solid & $1.23 \mathrm{E}+04$ & $1.42 \mathrm{E}+01$ & $3.78 \mathrm{E}+00$ & $1.61 \mathrm{E}+01$ & $1.04 \mathrm{E}+00$ & $1.87 \mathrm{E}+01$ \\
\hline $241-\mathrm{T}-101$ & Supernatant & $0.00 \mathrm{E}+00$ & $0.00 \mathrm{E}+00$ & $0.00 \mathrm{E}+00$ & $0.00 \mathrm{E}+00$ & $0.00 \mathrm{E}+00$ & $0.00 \mathrm{E}+00$ \\
\hline $241-\mathrm{T}-101$ & Solid & $6.01 \mathrm{E}-01$ & $1.17 \mathrm{E}-01$ & $7.82 \mathrm{E}-02$ & $3.58 \mathrm{E}-01$ & $9.08 \mathrm{E}-03$ & $6.17 \mathrm{E}+01$ \\
\hline $241-\mathrm{T}-102$ & Supernatant & $7.77 \mathrm{E}-01$ & $3.76 \mathrm{E}-03$ & $1.04 \mathrm{E}-03$ & $4.48 \mathrm{E}-03$ & $1.81 \mathrm{E}-04$ & $4.13 \mathrm{E}+01$ \\
\hline $241-\mathrm{T}-102$ & Solid & $1.16 \mathrm{E}+02$ & $1.63 \mathrm{E}-01$ & $6.88 \mathrm{E}-03$ & $2.95 \mathrm{E}-02$ & $6.65 \mathrm{E}-04$ & $1.50 \mathrm{E}+01$ \\
\hline $241-\mathrm{T}-103$ & Supernatant & $1.76 \mathrm{E}+00$ & $8.57 \mathrm{E}-03$ & $1.48 \mathrm{E}-03$ & $6.39 \mathrm{E}-03$ & $2.59 \mathrm{E}-04$ & $1.07 \mathrm{E}+01$ \\
\hline $241-\mathrm{T}-103$ & & $4.02 \mathrm{E}+00$ & $3.45 \mathrm{E}-03$ & $5.71 \mathrm{E}-03$ & $2.44 \mathrm{E}-02$ & $5.51 \mathrm{E}-04$ & $1.17 \mathrm{E}+00$ \\
\hline $241-\mathrm{T}-104$ & Supernatant & $0.00 \mathrm{E}+00$ & $0.00 \mathrm{E}+00$ & $0.00 \mathrm{E}+00$ & $0.00 \mathrm{E}+00$ & $0.00 \mathrm{E}+00$ & $0.00 \mathrm{E}+00$ \\
\hline $241-T-104$ & Solid & $1.84 \mathrm{E}+00$ & $1.83 \mathrm{E}-02$ & $1.51 \mathrm{E}-02$ & $1.25 \mathrm{E}-01$ & $1.58 \mathrm{E}-03$ & $1.42 \mathrm{E}-01$ \\
\hline 241-T-105 & Supernatant & $0.00 \mathrm{E}+00$ & $0.00 \mathrm{E}+00$ & $0.00 \mathrm{E}+00$ & $0.00 \mathrm{E}+00$ & $0.00 \mathrm{E}+00$ & $0.00 \mathrm{E}+00$ \\
\hline $241-\mathrm{T}-105$ & Solid & $4.72 \mathrm{E}+01$ & $5.84 \mathrm{E}-02$ & $2.93 \mathrm{E}-02$ & $2.77 \mathrm{E}-01$ & $2.01 \mathrm{E}-03$ & $8.42 \mathrm{E}+00$ \\
\hline $241-\mathrm{T}-106$ & Supernatant & $0.00 \mathrm{E}+00$ & $0.00 \mathrm{E}+00$ & $0.00 \mathrm{E}+00$ & $0.00 \mathrm{E}+00$ & $0.00 \mathrm{E}+00$ & $0.00 \mathrm{E}+00$ \\
\hline $241-\mathrm{T}-106$ & Solid & $4.57 \mathrm{E}+00$ & $4.27 \mathrm{E}-02$ & $2.00 \mathrm{E}-02$ & $1.34 \mathrm{E}-01$ & $1.56 \mathrm{E}-03$ & $1.40 \mathrm{E}+01$ \\
\hline $241-\mathrm{T}-107$ & Supernatant & $0.00 \mathrm{E}+00$ & $0.00 \mathrm{E}+00$ & $0.00 \mathrm{E}+00$ & $0.00 \mathrm{E}+00$ & $0.00 \mathrm{E}+00$ & $0.00 \mathrm{E}+00$ \\
\hline $241-\mathrm{T}-107$ & & $9.14 \mathrm{E}+01$ & $5.89 \mathrm{E}-02$ & $3.48 \mathrm{E}-02$ & 3.04E-01 & $2.29 \mathrm{E}-03$ & $1.34 \mathrm{E}+01$ \\
\hline $241-$ & $\tan t$ & $0.00 \mathrm{E}+00$ & $0.00 \mathrm{E}+00$ & $0.00 \mathrm{E}+00$ & $0.00 \mathrm{E}+00$ & $0.00 \mathrm{E}+00$ & $0.00 \mathrm{E}+00$ \\
\hline 241 & & $1.61 \mathrm{E}+00$ & 4.69E-03 & $6.74 \mathrm{E}-03$ & $6.05 \mathrm{E}-02$ & $4.25 \mathrm{E}-04$ & $6.17 \mathrm{E}+00$ \\
\hline $241-\mathrm{T}$ & Supe & $0.00 \mathrm{E}+00$ & $0.00 \mathrm{E}+00$ & $0.00 \mathrm{E}+00$ & $0.00 \mathrm{E}+00$ & $0.00 \mathrm{E}+00$ & $0.00 \mathrm{E}+00$ \\
\hline 241-T-109 & Solid & $2.38 \mathrm{E}-01$ & $2.19 \mathrm{E}-04$ & $1.04 \mathrm{E}-03$ & $9.12 \mathrm{E}-03$ & $6.58 \mathrm{E}-05$ & $1.94 \mathrm{E}+00$ \\
\hline $241-T-110$ & Supernatant & $3.90 \mathrm{E}-04$ & $2.89 \mathrm{E}-06$ & $9.97 \mathrm{E}-06$ & $1.48 \mathrm{E}-04$ & $1.83 \mathrm{E}-06$ & $2.55 \mathrm{E}-03$ \\
\hline $241-\mathrm{T}-110$ & Solid & $2.48 \mathrm{E}-02$ & $4.97 \mathrm{E}-03$ & $5.88 \mathrm{E}-03$ & $5.61 \mathrm{E}-02$ & $3.63 \mathrm{E}-04$ & $1.36 \mathrm{E}-02$ \\
\hline $241-\mathrm{T}-111$ & Supernatant & $0.00 \mathrm{E}+00$ & $0.00 \mathrm{E}+00$ & $0.00 \mathrm{E}+00$ & $0.00 \mathrm{E}+00$ & $0.00 \mathrm{E}+00$ & $0.00 \mathrm{E}+00$ \\
\hline $241-\mathrm{T}-111$ & Solid & $3.71 \mathrm{E}+00$ & $4.68 \mathrm{E}-02$ & $1.44 \mathrm{E}-02$ & $1.24 \mathrm{E}-01$ & $5.89 \mathrm{E}-04$ & $9.28 \mathrm{E}-02$ \\
\hline $241-\mathrm{T}-112$ & Supernatant & $5.52 \mathrm{E}-01$ & $1.03 \mathrm{E}-02$ & $1.78 \mathrm{E}-03$ & $7.69 \mathrm{E}-03$ & $3.12 \mathrm{E}-04$ & $3.37 \mathrm{E}+00$ \\
\hline $\mathrm{T}-112$ & & $1.81 \mathrm{E}-03$ & $1.53 \mathrm{E}-02$ & $2.41 \mathrm{E}-02$ & $2.14 \mathrm{E}-01$ & $1.53 \mathrm{E}-03$ & $3.52 \mathrm{E}-03$ \\
\hline 241-T-201 & upe & $3.21 \mathrm{E}-05$ & $6.34 \mathrm{E}$ & $1.67 \mathrm{E}$ & $2.49 \mathrm{E}-05$ & $8.15 \mathrm{E}$ & $3.71 \mathrm{E}-05$ \\
\hline $241-$ & & $6.11 \mathrm{E}-02$ & $3.96 \mathrm{E}-02$ & $4.52 \mathrm{E}-02$ & $6.71 \mathrm{E}-01$ & $2.20 \mathrm{E}-03$ & $2.02 \mathrm{E}-02$ \\
\hline 241-T-202 & upe & $0.00 \mathrm{E}+00$ & $0.00 \mathrm{E}+00$ & $0.00 \mathrm{E}+00$ & $0.00 \mathrm{E}+00$ & $0.00 \mathrm{E}+00$ & $0.00 \mathrm{E}+00$ \\
\hline 241-T-202 & & $2.04 \mathrm{E}-03$ & $3.22 \mathrm{E}-02$ & $2.11 \mathrm{E}-02$ & $1.67 \mathrm{E}-01$ & $1.40 \mathrm{E}-03$ & $6.17 \mathrm{E}-03$ \\
\hline 241-T-203 & upernatant & $0.00 \mathrm{E}+00$ & $0.00 \mathrm{E}+00$ & $0.00 \mathrm{E}+00$ & $0.00 \mathrm{E}+00$ & $0.00 \mathrm{E}+00$ & $0.00 \mathrm{E}+00$ \\
\hline $241-\mathrm{T}-203$ & Solid & $2.01 \mathrm{E}-03$ & $3.75 \mathrm{E}-02$ & $2.81 \mathrm{E}-02$ & $2.24 \mathrm{E}-01$ & $1.87 \mathrm{E}-03$ & $6.09 \mathrm{E}-03$ \\
\hline $241-\mathrm{T}-204$ & Supernatant & $0.00 \mathrm{E}+00$ & $0.00 \mathrm{E}+00$ & $0.00 \mathrm{E}+00$ & $0.00 \mathrm{E}+00$ & $0.00 \mathrm{E}+00$ & $0.00 \mathrm{E}+00$ \\
\hline $241-\mathrm{T}-204$ & & $3.66 \mathrm{E}-03$ & $2.28 \mathrm{E}-02$ & $2.39 \mathrm{E}-02$ & $1.89 \mathrm{E}-01$ & $1.59 \mathrm{E}-03$ & $6.23 \mathrm{E}-03$ \\
\hline 241-TX-101 & Supernatant & $0.00 \mathrm{E}+00$ & $0.00 \mathrm{E}+00$ & $0.00 \mathrm{E}+00$ & $0.00 \mathrm{E}+00$ & $0.00 \mathrm{E}+00$ & $0.00 \mathrm{E}+00$ \\
\hline 241-TX-101 & & $2.93 \mathrm{E}+02$ & $4.25 \mathrm{E}-01$ & $1.25 \mathrm{E}-01$ & $5.90 \mathrm{E}-01$ & $1.89 \mathrm{E}-02$ & $6.12 \mathrm{E}+01$ \\
\hline 241 & & $0.00 \mathrm{E}+00$ & $0.00 \mathrm{E}+00$ & $0.00 \mathrm{E}+00$ & $0.00 \mathrm{E}+00$ & $0.00 \mathrm{E}+00$ & $0.00 \mathrm{E}+00$ \\
\hline 241- & & $1.43 \mathrm{E}+00$ & $1.45 \mathrm{E}-01$ & $1.52 \mathrm{E}-02$ & $7.23 \mathrm{E}-02$ & $2.46 \mathrm{E}-03$ & $7.97 \mathrm{E}+01$ \\
\hline $241-T$ & natant & $0.00 \mathrm{E}+00$ & $0.00 \mathrm{E}+00$ & $0.00 \mathrm{E}+00$ & $0.00 \mathrm{E}+00$ & $0.00 \mathrm{E}+00$ & $0.00 \mathrm{E}+00$ \\
\hline 241-TX-103 & & $6.62 \mathrm{E}-01$ & $1.43 \mathrm{E}-01$ & $1.50 \mathrm{E}-02$ & $7.18 \mathrm{E}-02$ & $2.43 \mathrm{E}-03$ & $7.96 \mathrm{E}+01$ \\
\hline 241-TX-104 & upernatant & $5.89 \mathrm{E}-01$ & $8.80 \mathrm{E}-03$ & $8.10 \mathrm{E}-05$ & $3.86 \mathrm{E}-04$ & $1.32 \mathrm{E}-05$ & $2.11 E+02$ \\
\hline 241-TX-104 & Solid & $1.49 \mathrm{E}+02$ & $1.59 \mathrm{E}-01$ & $2.17 \mathrm{E}-02$ & $1.06 \mathrm{E}-01$ & $3.05 \mathrm{E}-03$ & $5.91 \mathrm{E}+01$ \\
\hline 241-TX-105 & Supernatant & $0.00 \mathrm{E}+00$ & $0.00 \mathrm{E}+00$ & $0.00 \mathrm{E}+00$ & $0.00 \mathrm{E}+00$ & $0.00 \mathrm{E}+00$ & $0.00 \mathrm{E}+00$ \\
\hline 241-TX-105 & Solid & $1.77 \mathrm{E}+00$ & $1.59 \mathrm{E}-01$ & 1.67E-02 & $7.95 \mathrm{E}-02$ & $2.71 \mathrm{E}-03$ & $6.47 \mathrm{E}+01$ \\
\hline 241-TX-106 & Supernatant & $0.00 \mathrm{E}+00$ & $0.00 \mathrm{E}+00$ & $0.00 \mathrm{E}+00$ & $0.00 \mathrm{E}+00$ & $0.00 \mathrm{E}+00$ & $0.00 \mathrm{E}+00$ \\
\hline 241-TX-106 & Solid & $5.52 \mathrm{E}+00$ & $1.53 \mathrm{E}-01$ & $1.68 \mathrm{E}-02$ & $8.06 \mathrm{E}-02$ & $2.64 \mathrm{E}-03$ & $7.53 \mathrm{E}+01$ \\
\hline 241-TX-107 & Supernatant & $0.00 \mathrm{E}+00$ & $0.00 \mathrm{E}+00$ & $0.00 \mathrm{E}+00$ & $0.00 \mathrm{E}+00$ & $0.00 \mathrm{E}+00$ & $0.00 \mathrm{E}+00$ \\
\hline 241-TX-107 & & $4.97 \mathrm{E}+00$ & $1.69 \mathrm{E}+00$ & $1.77 \mathrm{E}-01$ & $8.41 \mathrm{E}-01$ & $2.88 \mathrm{E}-02$ & $9.28 \mathrm{E}+01$ \\
\hline 241-TX-108 & 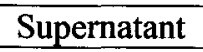 & $0.00 \mathrm{E}+00$ & $0.00 \mathrm{E}+00$ & $0.00 \mathrm{E}+00$ & $0.00 \mathrm{E}+00$ & $0.00 \mathrm{E}+00$ & $0.00 \mathrm{E}+00$ \\
\hline 241-TX-108 & Solid & $4.79 \mathrm{E}+00$ & $1.49 \mathrm{E}-01$ & $1.55 \mathrm{E}-02$ & $7.45 \mathrm{E}-02$ & $2.53 \mathrm{E}-03$ & $6.84 \mathrm{E}+01$ \\
\hline
\end{tabular}


Table A-2. Tank Waste Radionuclide Concentrations.

\begin{tabular}{|c|c|c|c|c|c|c|c|}
\hline Tank & Layer & $\begin{array}{c}{ }^{90} \mathrm{Sr} \\
(\mu \mathrm{Ci} / \mathrm{g})\end{array}$ & $\begin{array}{c}{ }^{241} \mathrm{Am} \\
(\mu \mathrm{Ci} / \mathrm{g})\end{array}$ & $\begin{array}{c}{ }^{240} \mathrm{Pu} \\
(\mu \mathrm{Ci} / \mathrm{g})\end{array}$ & $\begin{array}{c}{ }^{239} \mathrm{Pu} \\
(\mu \mathrm{Ci} / \mathrm{g})\end{array}$ & $\begin{array}{c}{ }^{238} \mathrm{Pu} \\
(\mu \mathrm{Ci} / \mathrm{g})\end{array}$ & $\begin{array}{c}{ }^{137} \mathrm{Cs} \\
(\mu \mathrm{Ci} / \mathrm{g})\end{array}$ \\
\hline 241-TX-109 & Supernatant & $0.00 \mathrm{E}+00$ & $0.00 \mathrm{E}+00$ & $0.00 \mathrm{E}+00$ & $0.00 \mathrm{E}+00$ & $0.00 \mathrm{E}+00$ & $0.00 \mathrm{E}+00$ \\
\hline 241-TX-109 & Solid & $4.38 \mathrm{E}+00$ & $1.38 \mathrm{E}-02$ & $9.05 \mathrm{E}-03$ & $8.34 \mathrm{E}-02$ & $5.70 \mathrm{E}-04$ & $1.44 \mathrm{E}+01$ \\
\hline $241-\mathrm{TX}-110$ & Supernatant & $0.00 \mathrm{E}+00$ & $0.00 \mathrm{E}+00$ & $0.00 \mathrm{E}+00$ & $0.00 \mathrm{E}+00$ & $0.00 \mathrm{E}+00$ & $0.00 \mathrm{E}+00$ \\
\hline $241-\mathrm{TX}-110$ & Solid & $9.33 \mathrm{E}-01$ & $1.54 \mathrm{E}-01$ & $1.66 \mathrm{E}-02$ & $8.20 \mathrm{E}-02$ & $2.64 \mathrm{E}-03$ & $5.97 \mathrm{E}+01$ \\
\hline $241-\mathrm{TX}-111$ & Supernatant & $0.00 \mathrm{E}+00$ & $0.00 \mathrm{E}+00$ & $0.00 \mathrm{E}+00$ & $0.00 \mathrm{E}+00$ & $0.00 \mathrm{E}+00$ & $0.00 \mathrm{E}+00$ \\
\hline 241-TX-111 & Solid & $1.06 \mathrm{E}+00$ & $1.51 \mathrm{E}-01$ & $1.65 \mathrm{E}-02$ & $8.28 \mathrm{E}-02$ & $2.59 \mathrm{E}-03$ & $5.67 \mathrm{E}+01$ \\
\hline 241-TX-112 & Supernatant & $0.00 \mathrm{E}+00$ & $0.00 \mathrm{E}+00$ & $0.00 \mathrm{E}+00$ & $0.00 \mathrm{E}+00$ & $0.00 \mathrm{E}+00$ & $0.00 \mathrm{E}+00$ \\
\hline $241-\mathrm{TX}-112$ & Solid & $6.60 \mathrm{E}-01$ & $1.57 \mathrm{E}-01$ & $1.65 \mathrm{E}-02$ & $7.94 \mathrm{E}-02$ & $2.66 \mathrm{E}-03$ & $6.22 \mathrm{E}+01$ \\
\hline 241-TX-113 & Supernatant & $0.00 \mathrm{E}+00$ & $0.00 \mathrm{E}+00$ & $0.00 \mathrm{E}+00$ & $0.00 \mathrm{E}+00$ & $0.00 \mathrm{E}+00$ & $0.00 \mathrm{E}+00$ \\
\hline 241-TX-113 & Solid & $1.27 \mathrm{E}+00$ & $6.72 \mathrm{E}-03$ & $1.68 \mathrm{E}-03$ & $1.32 \mathrm{E}-02$ & $1.57 \mathrm{E}-04$ & $7.48 \mathrm{E}+00$ \\
\hline $241-\mathrm{TX}-114$ & Supernatant & $0.00 \mathrm{E}+00$ & $0.00 \mathrm{E}+00$ & $0.00 \mathrm{E}+00$ & $0.00 \mathrm{E}+00$ & $0.00 \mathrm{E}+00$ & $0.00 \mathrm{E}+00$ \\
\hline 241-TX-114 & Solid & $6.61 \mathrm{E}-01$ & $1.48 \mathrm{E}-01$ & $1.59 \mathrm{E}-02$ & $7.75 \mathrm{E}-02$ & $2.54 \mathrm{E}-03$ & $5.42 \mathrm{E}+01$ \\
\hline $241-\mathrm{TX}-115$ & Supernatant & $0.00 \mathrm{E}+00$ & $0.00 \mathrm{E}+00$ & $0.00 \mathrm{E}+00$ & $0.00 \mathrm{E}+00$ & $0.00 \mathrm{E}+00$ & $0.00 \mathrm{E}+00$ \\
\hline $241-\mathrm{TX}-115$ & Solid & $1.96 \mathrm{E}+00$ & $1.60 \mathrm{E}-01$ & $1.67 \mathrm{E}-02$ & $7.97 \mathrm{E}-02$ & $2.72 \mathrm{E}-03$ & $6.51 \mathrm{E}+01$ \\
\hline 241-TX-116 & Supernatant & $0.00 \mathrm{E}+00$ & $0.00 \mathrm{E}+00$ & $0.00 \mathrm{E}+00$ & $0.00 \mathrm{E}+00$ & $0.00 \mathrm{E}+00$ & $0.00 \mathrm{E}+00$ \\
\hline 241-TX-116 & Solid & $3.10 \mathrm{E}-01$ & $6.88 \mathrm{E}-02$ & $9.33 \mathrm{E}-03$ & $5.34 \mathrm{E}-02$ & $1.30 \mathrm{E}-03$ & $3.20 \mathrm{E}+01$ \\
\hline $241-\mathrm{TX}-117$ & Supern & $0.00 \mathrm{E}+00$ & $0.00 \mathrm{E}+00$ & $0.00 \mathrm{E}+00$ & $0.00 \mathrm{E}+00$ & $0.00 \mathrm{E}+00$ & $0.00 \mathrm{E}+00$ \\
\hline 241-TX-117 & Solid & $4.63 \mathrm{E}-01$ & $1.00 \mathrm{E}-01$ & $1.20 \mathrm{E}-02$ & $6.32 \mathrm{E}-02$ & $1.79 \mathrm{E}-03$ & $3.54 \mathrm{E}+01$ \\
\hline 241-TX-118 & Supernatant & $0.00 \mathrm{E}+00$ & $0.00 \mathrm{E}+00$ & $0.00 \mathrm{E}+00$ & $0.00 \mathrm{E}+00$ & $0.00 \mathrm{E}+00$ & $0.00 \mathrm{E}+00$ \\
\hline 241-TX-118 & Solid & $1.10 \mathrm{E}+02$ & $3.61 \mathrm{E}+00$ & $4.93 \mathrm{E}-01$ & $2.34 \mathrm{E}+00$ & $8.03 \mathrm{E}-02$ & $4.92 \mathrm{E}+01$ \\
\hline 241-TY-101 & Supernatant & $0.00 \mathrm{E}+00$ & $0.00 \mathrm{E}+00$ & $0.00 \mathrm{E}+00$ & $0.00 \mathrm{E}+00$ & $0.00 \mathrm{E}+00$ & $0.00 \mathrm{E}+00$ \\
\hline 241-TY-101 & & $8.66 \mathrm{E}+00$ & $1.27 \mathrm{E}-02$ & $1.44 \mathrm{E}-02$ & $1.72 \mathrm{E}-01$ & $8.20 \mathrm{E}-04$ & $3.68 \mathrm{E}-01$ \\
\hline $241-\mathrm{TY}-102$ & Supern & $0.00 \mathrm{E}+00$ & $0.00 \mathrm{E}+00$ & $0.00 \mathrm{E}+00$ & $0.00 \mathrm{E}+00$ & $0.00 \mathrm{E}+00$ & $0.00 \mathrm{E}+00$ \\
\hline $241-\mathrm{T}$ & & $2.38 \mathrm{E}+00$ & $2.49 \mathrm{E}-03$ & $2.02 \mathrm{E}-04$ & $1.33 \mathrm{E}-03$ & $2.39 \mathrm{E}-05$ & $2.61 \mathrm{E}+01$ \\
\hline $241-\mathrm{TY}-103$ & Supernatant & $0.00 \mathrm{E}+00$ & $0.00 \mathrm{E}+00$ & $0.00 \mathrm{E}+00$ & $0.00 \mathrm{E}+00$ & $0.00 \mathrm{E}+00$ & $0.00 \mathrm{E}+00$ \\
\hline 241-TY-103 & & $6.37 \mathrm{E}+01$ & $2.45 \mathrm{E}-02$ & $1.93 \mathrm{E}-02$ & $1.60 \mathrm{E}-01$ & $2.03 \mathrm{E}-03$ & $2.54 \mathrm{E}+01$ \\
\hline $241-\mathrm{TY}-104$ & Supernatant & $4.66 \mathrm{E}-02$ & $3.78 \mathrm{E}-04$ & $2.64 \mathrm{E}-04$ & $1.11 \mathrm{E}-03$ & $6.07 \mathrm{E}-05$ & $8.86 \mathrm{E}+00$ \\
\hline $241-\mathrm{TY}-104$ & Solid & $7.87 \mathrm{E}+01$ & $7.93 \mathrm{E}-02$ & $1.36 \mathrm{E}-02$ & $1.72 \mathrm{E}-01$ & $7.57 \mathrm{E}-04$ & $3.21 \mathrm{E}+01$ \\
\hline 241-TY-105 & Supernatant & $0.00 \mathrm{E}+00$ & $0.00 \mathrm{E}+00$ & $0.00 \mathrm{E}+00$ & $0.00 \mathrm{E}+00$ & $0.00 \mathrm{E}+00$ & $0.00 \mathrm{E}+00$ \\
\hline 241-TY-105 & Solid & $1.34 \mathrm{E}+02$ & $7.55 \mathrm{E}-03$ & $2.27 \mathrm{E}-03$ & $2.12 \mathrm{E}-02$ & $1.43 \mathrm{E}-04$ & $5.69 \mathrm{E}+00$ \\
\hline $241-1$ & atant & $0.00 \mathrm{E}+00$ & $0.00 \mathrm{E}+00$ & $0.00 \mathrm{E}+00$ & $0.00 \mathrm{E}+00$ & $0.00 \mathrm{E}+00$ & $0.00 \mathrm{E}+00$ \\
\hline 241-TY-107 & & $1.10 \mathrm{E}+02$ & $1.66 \mathrm{E}-02$ & $2.04 \mathrm{E}-03$ & $1.90 \mathrm{E}-02$ & $1.28 \mathrm{E}-04$ & $5.15 \mathrm{E}+01$ \\
\hline $241-U-101$ & Supernatant & $0.00 \mathrm{E}+00$ & $0.00 \mathrm{E}+00$ & $0.00 \mathrm{E}+00$ & $0.00 \mathrm{E}+00$ & $0.00 \mathrm{E}+00$ & $0.00 \mathrm{E}+00$ \\
\hline 241-U-101 & & $3.38 \mathrm{E}+02$ & $2.05 \mathrm{E}-02$ & $7.86 \mathrm{E}-03$ & $4.06 \mathrm{E}-02$ & $7.27 \mathrm{E}-04$ & $5.22 \mathrm{E}+01$ \\
\hline $241-\mathrm{U}-102$ & Supernatant & $4.14 E+00$ & $7.80 \mathrm{E}-03$ & $6.59 \mathrm{E}-05$ & $3.14 \mathrm{E}-04$ & $1.08 \mathrm{E}-05$ & $2.77 \mathrm{E}+02$ \\
\hline $241-\mathrm{U}-102$ & Solid & $7.67 \mathrm{E}+01$ & $1.18 \mathrm{E}-01$ & $2.30 \mathrm{E}-02$ & $1.14 \mathrm{E}-01$ & $2.79 \mathrm{E}-03$ & $1.28 \mathrm{E}+02$ \\
\hline $241-U-103$ & Supernatant & $5.90 \mathrm{E}+00$ & $1.28 \mathrm{E}-02$ & $5.97 \mathrm{E}-05$ & $2.74 \mathrm{E}-04$ & $9.34 \mathrm{E}-06$ & $2.82 \mathrm{E}+02$ \\
\hline $241-U-103$ & Solid & $2.08 \mathrm{E}+01$ & $8.50 \mathrm{E}-02$ & $9.06 \mathrm{E}-03$ & $4.31 \mathrm{E}-02$ & $1.27 \mathrm{E}-03$ & $1.00 \mathrm{E}+02$ \\
\hline 241-U-104 & Supernatant & $0.00 \mathrm{E}+00$ & $0.00 \mathrm{E}+00$ & $0.00 \mathrm{E}+00$ & $0.00 \mathrm{E}+00$ & $0.00 \mathrm{E}+00$ & $0.00 \mathrm{E}+00$ \\
\hline 241-U-104 & Solid & $2.29 \mathrm{E}+00$ & $1.27 \mathrm{E}-03$ & $6.00 \mathrm{E}-04$ & $4.74 \mathrm{E}-03$ & $3.97 \mathrm{E}-05$ & $2.01 \mathrm{E}-01$ \\
\hline $241-U-105$ & Supernatant & $0.00 \mathrm{E}+00$ & $0.00 \mathrm{E}+00$ & $0.00 \mathrm{E}+00$ & $0.00 \mathrm{E}+00$ & $0.00 \mathrm{E}+00$ & $0.00 \mathrm{E}+00$ \\
\hline 241-U-105 & & $1.03 \mathrm{E}+01$ & $3.62 \mathrm{E}-01$ & $5.41 \mathrm{E}-02$ & $2.59 \mathrm{E}-01$ & $7.70 \mathrm{E}-03$ & $1.24 \mathrm{E}+02$ \\
\hline $241-\mathrm{U}-106$ & Supernatant & $3.73 \mathrm{E}+01$ & $2.14 \mathrm{E}-01$ & $9.19 \mathrm{E}-04$ & $4.22 \mathrm{E}-03$ & $1.44 \mathrm{E}-04$ & $1.92 \mathrm{E}+02$ \\
\hline $241-U-106$ & Solid & $5.40 \mathrm{E}+01$ & $6.83 \mathrm{E}-01$ & $4.35 \mathrm{E}-02$ & $2.00 \mathrm{E}-01$ & $6.80 \mathrm{E}-03$ & $1.32 \mathrm{E}+02$ \\
\hline $241-U-107$ & Supernatant & $0.00 \mathrm{E}+00$ & $0.00 \mathrm{E}+00$ & $0.00 \mathrm{E}+00$ & $0.00 \mathrm{E}+00$ & $0.00 \mathrm{E}+00$ & $0.00 \mathrm{E}+00$ \\
\hline 241-U-107 & Solid & $1.90 \mathrm{E}+00$ & $2.15 \mathrm{E}-01$ & $3.04 \mathrm{E}-02$ & $1.42 \mathrm{E}-01$ & $4.80 \mathrm{E}-03$ & $6.90 \mathrm{E}+01$ \\
\hline $241-U-108$ & Supernatant & $0.00 \mathrm{E}+00$ & $0.00 \mathrm{E}+00$ & $0.00 \mathrm{E}+00$ & $0.00 \mathrm{E}+00$ & $0.00 \mathrm{E}+00$ & $0.00 \mathrm{E}+00$ \\
\hline 241-U-108 & Solid & $6.87 \mathrm{E}+00$ & $3.45 \mathrm{E}-02$ & $1.43 \mathrm{E}-02$ & $6.53 \mathrm{E}-02$ & $1.71 \mathrm{E}-03$ & $1.12 \mathrm{E}+02$ \\
\hline $241-U-109$ & Supernatant & $0.00 \mathrm{E}+00$ & $0.00 \mathrm{E}+00$ & $0.00 \mathrm{E}+00$ & $0.00 \mathrm{E}+00$ & $0.00 \mathrm{E}+00$ & $0.00 \mathrm{E}+00$ \\
\hline 241-U-109 & Solid & $6.31 \mathrm{E}+00$ & $2.35 \mathrm{E}-02$ & $2.37 \mathrm{E}-03$ & $1.11 \mathrm{E}-02$ & $3.56 \mathrm{E}-04$ & $1.03 \mathrm{E}+02$ \\
\hline
\end{tabular}


Table A-2. Tank Waste Radionuclide Concentrations.

\begin{tabular}{|c|c|c|c|c|c|c|c|}
\hline Tank & Layer & $\begin{array}{c}{ }^{90} \mathrm{Sr} \\
(\mu \mathrm{Ci} / \mathrm{g})\end{array}$ & $\begin{array}{c}{ }^{241} \mathrm{Am} \\
(\mu \mathrm{Ci} / \mathrm{g})\end{array}$ & $\begin{array}{c}{ }^{240} \mathrm{Pu} \\
(\mu \mathrm{Ci} / \mathrm{g})\end{array}$ & $\begin{array}{c}{ }^{239} \mathrm{Pu} \\
(\mu \mathrm{Ci} / \mathrm{g})\end{array}$ & $\begin{array}{c}{ }^{238} \mathrm{Pu} \\
(\mu \mathrm{Ci} / \mathrm{g})\end{array}$ & $\begin{array}{c}{ }^{137} \mathrm{Cs} \\
(\mu \mathrm{Ci} / \mathrm{g})\end{array}$ \\
\hline $241-\mathrm{U}-110$ & Supernatant & $0.00 \mathrm{E}+00$ & $0.00 \mathrm{E}+00$ & $0.00 \mathrm{E}+00$ & $0.00 \mathrm{E}+00$ & $0.00 \mathrm{E}+00$ & $0.00 \mathrm{E}+00$ \\
\hline $241-\mathrm{U}-110$ & Solid & $1.47 \mathrm{E}+02$ & $7.36 \mathrm{E}-02$ & $3.16 \mathrm{E}-02$ & $1.84 \mathrm{E}-01$ & $2.74 \mathrm{E}-03$ & $1.65 \mathrm{E}+01$ \\
\hline $241-\mathrm{U}-111$ & Supernatant & $0.00 \mathrm{E}+00$ & $0.00 \mathrm{E}+00$ & $0.00 \mathrm{E}+00$ & $0.00 \mathrm{E}+00$ & $0.00 \mathrm{E}+00$ & $0.00 \mathrm{E}+00$ \\
\hline $241-\mathrm{U}-111$ & Solid & $3.41 \mathrm{E}+01$ & $1.12 \mathrm{E}-01$ & $1.85 \mathrm{E}-02$ & $9.12 \mathrm{E}-02$ & $2.48 \mathrm{E}-03$ & $1.14 \mathrm{E}+02$ \\
\hline $241-\mathrm{U}-112$ & Supernatant & $0.00 \mathrm{E}+00$ & $0.00 \mathrm{E}+00$ & $0.00 \mathrm{E}+00$ & $0.00 \mathrm{E}+00$ & $0.00 \mathrm{E}+00$ & $0.00 \mathrm{E}+00$ \\
\hline $241-\mathrm{U}-112$ & Solid & $1.50 \mathrm{E}+02$ & $3.36 \mathrm{E}-03$ & $2.11 \mathrm{E}-03$ & $1.91 \mathrm{E}-02$ & $1.34 \mathrm{E}-04$ & $2.74 \mathrm{E}+01$ \\
\hline $241-\mathrm{U}-201$ & Supernatant & $9.19 \mathrm{E}-03$ & $4.31 \mathrm{E}-04$ & $3.45 \mathrm{E}-05$ & $1.82 \mathrm{E}-04$ & $2.74 \mathrm{E}-06$ & $1.97 \mathrm{E}+01$ \\
\hline $241-\mathrm{U}-201$ & Solid & $1.31 \mathrm{E}-01$ & $1.53 \mathrm{E}-03$ & $3.52 \mathrm{E}-04$ & $1.86 \mathrm{E}-03$ & $2.80 \mathrm{E}-05$ & $4.31 \mathrm{E}+00$ \\
\hline $241-\mathrm{U}-202$ & Supernatant & $4.38 \mathrm{E}-04$ & $7.19 \mathrm{E}-06$ & $8.73 \mathrm{E}-07$ & $4.59 \mathrm{E}-06$ & $6.91 \mathrm{E}-08$ & $1.39 \mathrm{E}+01$ \\
\hline $241-\mathrm{U}-202$ & Solid & $5.60 \mathrm{E}-02$ & $1.89 \mathrm{E}-03$ & $4.93 \mathrm{E}-04$ & $2.59 \mathrm{E}-03$ & $3.91 \mathrm{E}-05$ & $3.77 \mathrm{E}+00$ \\
\hline $241-\mathrm{U}-203$ & Supernatant & $3.85 \mathrm{E}-04$ & $6.95 \mathrm{E}-06$ & $3.71 \mathrm{E}-06$ & $1.95 \mathrm{E}-05$ & $2.93 \mathrm{E}-07$ & $1.31 \mathrm{E}+01$ \\
\hline $241-\mathrm{U}-203$ & Solid & $1.15 \mathrm{E}-01$ & $1.54 \mathrm{E}-03$ & $2.88 \mathrm{E}-04$ & $1.52 \mathrm{E}-03$ & $2.29 \mathrm{E}-05$ & $3.55 \mathrm{E}+00$ \\
\hline $241-\mathrm{U}-204$ & Supernatant & $1.61 \mathrm{E}-04$ & $8.47 \mathrm{E}-06$ & $6.69 \mathrm{E}-07$ & $3.54 \mathrm{E}-06$ & $5.32 \mathrm{E}-08$ & $5.59 \mathrm{E}+00$ \\
\hline $241-\mathrm{U}-204$ & Solid & $3.46 \mathrm{E}+00$ & $2.22 \mathrm{E}-02$ & $1.21 \mathrm{E}-02$ & $6.34 \mathrm{E}-02$ & $9.56 \mathrm{E}-04$ & $4.54 \mathrm{E}+00$ \\
\hline
\end{tabular}

Table A-3. Tank Waste Physical Properties.

\begin{tabular}{|c|c|c|c|c|c|c|c|c|}
\hline Tank & Layer & $\begin{array}{l}\text { Bulk } \\
\text { SpG }\end{array}$ & $\begin{array}{l}\text { Liquid } \\
\text { SpG }\end{array}$ & \begin{tabular}{|c|} 
Non-RG \\
Waste Volume \\
(kL)
\end{tabular} & $\begin{array}{c}\text { Water in } \\
\text { Bulk } \\
(w t \%) \\
\end{array}$ & $\begin{array}{l}\text { Water in } \\
\text { Liquid } \\
\text { (wt\%) }\end{array}$ & $\begin{array}{c}\text { T }_{\text {Waste }} \\
\left({ }^{\circ} \mathrm{C}\right)\end{array}$ & $\begin{array}{c}\mathbf{T}_{\text {Headspace }} \\
\left({ }^{\circ} \mathrm{C}\right)\end{array}$ \\
\hline $241-\mathrm{AN}-101$ & Supernatant & 1.40 & 1.40 & 4208 & $52.1 \%$ & $52.1 \%$ & 26 & 23 \\
\hline 241-AN-101 & Solid & 1.55 & 1.45 & 118 & $40.3 \%$ & $49.1 \%$ & 26 & 23 \\
\hline $241-\mathrm{AN}-102$ & Supernatant & 1.41 & 1.41 & 3446 & $48.9 \%$ & $48.9 \%$ & 30 & 28 \\
\hline 241-AN-102 & Solid & 1.53 & 1.41 & 584 & $39.9 \%$ & $48.9 \%$ & 30 & 28 \\
\hline 241-AN-103 & Supernatant & 1.48 & 1.48 & 1769 & $46.7 \%$ & $46.7 \%$ & 38 & 31 \\
\hline 241-AN-103 & Solid & 1.52 & 1.48 & 1858 & $34.9 \%$ & $46.7 \%$ & 38 & 31 \\
\hline 241-AN-104 & Supernatant & 1.40 & 1.40 & 2298 & $51.1 \%$ & $51.1 \%$ & 37 & 31 \\
\hline 241-AN-104 & Solid & 1.48 & 1.40 & 1685 & $45.7 \%$ & $51.1 \%$ & 37 & 31 \\
\hline 241-AN-105 & Supernatant & 1.42 & 1.42 & 2227 & $49.9 \%$ & $49.9 \%$ & 34 & 29 \\
\hline 241-AN-105 & Solid & 1.49 & 1.42 & 2034 & $44.4 \%$ & $49.9 \%$ & 34 & 29 \\
\hline 241-AN-106 & Supernatant & 1.16 & 1.16 & 1430 & $80.1 \%$ & $80.1 \%$ & 48 & 29 \\
\hline 241-AN-106 & Solid & 1.50 & 1.16 & 1469 & $39.1 \%$ & $80.1 \%$ & 48 & 29 \\
\hline $241-\mathrm{AN}-107$ & Supernatant & 1.43 & 1.43 & 3297 & $51.5 \%$ & $51.5 \%$ & 32 & 27 \\
\hline 241-AN-107 & Solid & 1.48 & 1.43 & 872 & $44.3 \%$ & $51.5 \%$ & 32 & 27 \\
\hline 241-AP-101 & Supernatant & 1.24 & 1.24 & 4363 & $69.6 \%$ & $69.6 \%$ & 29 & 29 \\
\hline 241-AP-101 & Solid & 0.00 & 1.24 & 0 & $0.0 \%$ & $0.0 \%$ & 29 & 29 \\
\hline 241-AP-102 & Supernatant & 1.37 & 1.37 & 4004 & $55.0 \%$ & $55.0 \%$ & 24 & 22 \\
\hline 241-AP-102 & Solid & 1.73 & 1.37 & 105 & $36.7 \%$ & $55.0 \%$ & 24 & 22 \\
\hline 241-AP-103 & Supernatant & 1.38 & 1.38 & 4097 & $55.9 \%$ & $55.9 \%$ & 24 & 22 \\
\hline 241-AP-103 & Solid & 1.50 & 1.38 & 198 & $52.7 \%$ & $55.9 \%$ & 24 & 22 \\
\hline 241-AP-104 & Supernatant & 1.41 & 1.41 & 1789 & $54.1 \%$ & $54.1 \%$ & 47 & 30 \\
\hline 241-AP-104 & Solid & 1.61 & 1.41 & 68 & $43.1 \%$ & $54.1 \%$ & 47 & 30 \\
\hline 241-AP-105 & Supernatant & 1.23 & 1.23 & 3316 & $71.5 \%$ & $71.5 \%$ & 28 & 28 \\
\hline 241-AP-105 & Solid & 1.61 & 1.23 & 396 & $47.7 \%$ & $71.5 \%$ & 28 & 28 \\
\hline 241-AP-106 & Supernatant & 1.21 & 1.21 & 4306 & $72.4 \%$ & $72.4 \%$ & 29 & 27 \\
\hline 241-AP-106 & Solid & 0.00 & 1.21 & 0 & $0.0 \%$ & $72.4 \%$ & 29 & 27 \\
\hline 241-AP-107 & Supernatant & 1.24 & 1.24 & 4245 & $72.0 \%$ & $72.0 \%$ & 27 & 27 \\
\hline 241-AP-107 & Solid & 0.00 & 1.24 & 0 & $0.0 \%$ & $0.0 \%$ & 27 & 27 \\
\hline 241-AP-108 & Supernatant & 1.42 & 1.42 & 4290 & $51.9 \%$ & $51.9 \%$ & 27 & 27 \\
\hline 241-AP-108 & Solid & 1.57 & 1.42 & 425 & $44.6 \%$ & $51.9 \%$ & 27 & 27 \\
\hline
\end{tabular}


Table A-3. Tank Waste Physical Properties.

\begin{tabular}{|c|c|c|c|c|c|c|c|c|}
\hline Tank & Layer & $\begin{array}{l}\text { Bulk } \\
\text { SpG }\end{array}$ & $\begin{array}{l}\text { Liquid } \\
\text { SpG }\end{array}$ & $\begin{array}{c}\text { Non-RG } \\
\text { Waste Volume } \\
(\mathbf{k L})\end{array}$ & $\begin{array}{c}\text { Water in } \\
\text { Bulk } \\
\text { (wt\%) }\end{array}$ & $\begin{array}{l}\text { Water in } \\
\text { Liquid } \\
\text { (wt\%) }\end{array}$ & $\begin{array}{l}T_{\text {Waste }} \\
\left({ }^{\circ} \mathbf{C}\right)\end{array}$ & $\begin{array}{c}\mathbf{T}_{\text {Headspace }} \\
\left({ }^{\circ} \mathbf{C}\right)\end{array}$ \\
\hline 241-AW-101 & Supernatant & 1.47 & 1.47 & 2770 & $43.8 \%$ & $43.8 \%$ & 37 & 30 \\
\hline 241-AW-101 & Solid & 1.49 & 1.47 & 1498 & $43.9 \%$ & $43.8 \%$ & 37 & 30 \\
\hline 241-AW-102 & Supernatant & 1.23 & 1.23 & 3816 & $70.9 \%$ & $70.9 \%$ & 26 & 24 \\
\hline 241-AW-102 & Solid & 1.60 & 1.23 & 184 & $33.4 \%$ & $70.9 \%$ & 26 & 24 \\
\hline 241-AW-103 & Supernatant & 1.24 & 1.24 & 2930 & $69.1 \%$ & $69.1 \%$ & 24 & 22 \\
\hline $241-A W-103$ & Solid & 1.49 & 1.42 & 1211 & $54.5 \%$ & $50.5 \%$ & 24 & 22 \\
\hline 241-AW-104 & Supernatant & 1.36 & 1.36 & 3070 & $66.6 \%$ & $66.6 \%$ & 25 & 23 \\
\hline 241-AW-104 & Solid & 1.46 & 1.40 & 963 & $51.2 \%$ & $57.0 \%$ & 25 & 23 \\
\hline 241-AW-105 & Supernatant & 1.06 & 1.06 & 631 & $90.4 \%$ & $90.4 \%$ & 20 & 20 \\
\hline 241-AW-105 & Solid & 1.10 & 1.06 & 940 & $63.3 \%$ & $90.4 \%$ & 20 & 20 \\
\hline 241-AW-106 & Supernatant & 1.21 & 1.21 & 3240 & $72.9 \%$ & $72.9 \%$ & 33 & 25 \\
\hline 241-AW-106 & Solid & 1.77 & 1.21 & 1062 & $29.7 \%$ & $72.9 \%$ & 33 & 25 \\
\hline 241-AY-101 & Supernatant & 1.10 & 1.10 & 3244 & $83.8 \%$ & $83.8 \%$ & 46 & 29 \\
\hline 241-AY-101 & Solid & 1.78 & 1.29 & 398 & $30.0 \%$ & $83.8 \%$ & 46 & 29 \\
\hline 241-AY-102 & Supernatant & 1.30 & 1.30 & 3049 & $63.3 \%$ & $63.3 \%$ & 64 & 41 \\
\hline 241-AY-102 & Solid & 1.30 & 1.15 & 571 & $39.2 \%$ & $82.0 \%$ & 64 & 41 \\
\hline 241-AZ-101 & Supernatant & 1.23 & 1.23 & 3592 & $72.7 \%$ & $72.7 \%$ & 72 & 59 \\
\hline $241-\mathrm{AZ}-101$ & Solid & 1.59 & 1.23 & 197 & $35.6 \%$ & $72.7 \%$ & 72 & 59 \\
\hline 241-AZ-102 & Supernatant & 1.15 & 1.15 & 289 & $82.3 \%$ & $82.3 \%$ & 68 & 47 \\
\hline $241-\mathrm{AZ}-102$ & Solid & 1.41 & 1.15 & 396 & $54.8 \%$ & $82.3 \%$ & 68 & 47 \\
\hline 241-SY-101 & Supernatant & 1.13 & 1.13 & 3231 & $81.9 \%$ & $81.9 \%$ & 23 & 14 \\
\hline 241-SY-101 & Solid & 1.51 & 1.13 & 964 & $41.5 \%$ & $81.9 \%$ & 23 & 14 \\
\hline 241-SY-102 & Supernatant & 1.12 & 1.12 & 1384 & $82.1 \%$ & $82.1 \%$ & 23 & 21 \\
\hline 241-SY-102 & Solid & 1.30 & 1.65 & 769 & $47.1 \%$ & $66.1 \%$ & 23 & 21 \\
\hline 241-SY-103 & Supernatant & 1.49 & 1.49 & 1444 & $47.1 \%$ & $47.1 \%$ & 32 & 28 \\
\hline 241-SY-103 & Solid & 1.55 & 1.49 & 1353 & $36.9 \%$ & $47.1 \%$ & 32 & 28 \\
\hline $241-\mathrm{A}-101$ & Supernatant & 0.00 & 0.00 & 0 & $46.9 \%$ & $46.9 \%$ & 43 & 35 \\
\hline 241-A-101 & Solid & 1.46 & 1.49 & 1211 & $31.6 \%$ & $46.9 \%$ & 43 & 35 \\
\hline $241-\mathrm{A}-102$ & Supernatant & 1.57 & 1.57 & 12 & $46.2 \%$ & $46.2 \%$ & 36 & 34 \\
\hline $241-\mathrm{A}-102$ & Solid & 1.67 & 1.57 & 139 & $37.0 \%$ & $46.2 \%$ & 36 & 34 \\
\hline $241-A-103$ & Supernatant & 1.51 & 1.51 & 17 & $50.3 \%$ & $50.3 \%$ & 36 & 34 \\
\hline $241-A-103$ & Solid & 1.37 & 1.51 & 1417 & $41.3 \%$ & $50.3 \%$ & 36 & 34 \\
\hline 241-A-104 & Supernatant & 0.00 & 0.00 & 0 & $0.0 \%$ & $0.0 \%$ & 0 & 34 \\
\hline $241-A-104$ & Solid & 0.95 & 1.17 & 106 & $0.0 \%$ & $75.0 \%$ & 74 & 34 \\
\hline 241-A-105 & Supernatant & 0.00 & 0.00 & 0 & $0.0 \%$ & $0.0 \%$ & 54 & 54 \\
\hline 241-A-105 & Solid & 1.54 & 1.17 & 139 & $0.0 \%$ & $0.0 \%$ & 54 & 54 \\
\hline 241-A-106 & Supernatant & 0.00 & 0.00 & 0 & $0.0 \%$ & $0.0 \%$ & 58 & 58 \\
\hline $241-A-106$ & Solid & 1.70 & 1.17 & 299 & $34.3 \%$ & $75.0 \%$ & 58 & 58 \\
\hline 241-AX-101 & Supernatant & 0.00 & 0.00 & 0 & $43.1 \%$ & $43.1 \%$ & 42 & 28 \\
\hline 241-AX-101 & Solid & 1.70 & 1.53 & 1353 & $32.7 \%$ & $43.1 \%$ & 42 & 28 \\
\hline $241-\mathrm{AX}-102$ & Supernatant & 0.00 & 0.00 & 0 & $0.0 \%$ & $0.0 \%$ & 24 & 24 \\
\hline 241-AX-102 & Solid & 1.58 & 1.39 & 113 & $35.6 \%$ & $51.0 \%$ & 24 & 24 \\
\hline 241-AX-103 & Supernatant & 0.00 & 0.00 & 0 & $49.1 \%$ & $49.1 \%$ & 38 & 30 \\
\hline 241-AX-103 & Solid & 1.58 & 1.45 & 403 & $43.3 \%$ & $49.1 \%$ & 38 & 30 \\
\hline 241-AX-104 & Supernatant & 0.00 & 0.00 & 0 & $0.0 \%$ & $0.0 \%$ & 34 & 34 \\
\hline 241-AX-104 & Solid & 1.80 & 1.17 & 28 & $8.2 \%$ & $75.0 \%$ & 34 & 34 \\
\hline 241-B-101 & Supernatant & 0.00 & 0.00 & 0 & $50.0 \%$ & $50.0 \%$ & 37 & 31 \\
\hline 241-B-101 & Solid & 1.51 & 1.53 & 411 & $40.4 \%$ & $50.0 \%$ & 37 & 31 \\
\hline 241-B-102 & Supernatant & 1.26 & 1.26 & 15 & $69.6 \%$ & $69.6 \%$ & 20 & 20 \\
\hline $241-\mathrm{B}-102$ & Solid & 1.61 & 1.26 & 106 & $45.2 \%$ & $69.6 \%$ & 20 & 20 \\
\hline
\end{tabular}


Table A-3. Tank Waste Physical Properties.

\begin{tabular}{|c|c|c|c|c|c|c|c|c|}
\hline Tank & Layer & $\begin{array}{l}\text { Bulk } \\
\text { SpG }\end{array}$ & $\begin{array}{l}\text { Liquid } \\
\text { SpG }\end{array}$ & \begin{tabular}{|c|} 
Non-RG \\
Waste Volume \\
(kL)
\end{tabular} & $\begin{array}{c}\text { Water in } \\
\text { Bulk } \\
\text { (wt\%) }\end{array}$ & $\begin{array}{l}\text { Water in } \\
\text { Liquid } \\
\text { (wt\%) }\end{array}$ & $\begin{array}{l}\mathbf{T}_{\text {Waste }} \\
\left({ }^{\circ} \mathbf{C}\right)\end{array}$ & $\begin{array}{c}\mathbf{T}_{\text {Headspace }} \\
\left({ }^{\circ} \mathbf{C}\right)\end{array}$ \\
\hline 241-B-103 & Supernatant & 0.00 & 0.00 & 0 & $69.6 \%$ & $69.6 \%$ & 19 & 19 \\
\hline 241-B-103 & Solid & 1.61 & 1.26 & 211 & $49.5 \%$ & $69.6 \%$ & 19 & 19 \\
\hline 241-B-104 & Supernatant & 0.00 & 0.00 & 0 & $69.6 \%$ & $69.6 \%$ & 22 & 20 \\
\hline 241-B-104 & Solid & 1.38 & 1.26 & 1417 & $47.5 \%$ & $69.6 \%$ & 22 & 20 \\
\hline 241-B-105 & Supernatant & 0.00 & 0.00 & 0 & $69.6 \%$ & $69.6 \%$ & 25 & 25 \\
\hline 241-B-105 & Solid & 1.65 & 1.26 & 1098 & $43.3 \%$ & $69.6 \%$ & 25 & 25 \\
\hline 241-B-106 & Supernatant & 1.26 & 1.26 & 4 & $67.2 \%$ & $67.2 \%$ & 20 & 20 \\
\hline 241-B-106 & Solid & 1.38 & 1.26 & 460 & $59.9 \%$ & $67.2 \%$ & 20 & 20 \\
\hline $241-B-107$ & Supernatant & 0.00 & 0.00 & 0 & $57.5 \%$ & $57.5 \%$ & 24 & 24 \\
\hline $241-\mathrm{B}-107$ & Solid & 1.63 & 1.34 & 611 & $40.1 \%$ & $57.5 \%$ & 24 & 24 \\
\hline $241-\mathrm{B}-108$ & Supernatant & 0.00 & 0.00 & 0 & $69.6 \%$ & $69.6 \%$ & 20 & 20 \\
\hline 241-B-108 & Solid & 1.70 & 1.38 & 350 & $34.5 \%$ & $69.6 \%$ & 20 & 20 \\
\hline 241-B-109 & Supernatant & 0.00 & 0.00 & 0 & $69.6 \%$ & $69.6 \%$ & 19 & 19 \\
\hline 241-B-109 & Solid & 1.82 & 1.26 & 475 & $39.4 \%$ & $69.6 \%$ & 19 & 19 \\
\hline $241-B-110$ & Supernatant & 1.19 & 1.19 & 4 & $76.2 \%$ & $76.2 \%$ & 21 & 21 \\
\hline 241-B-110 & Solid & 1.36 & 1.19 & 925 & $58.3 \%$ & $76.2 \%$ & 21 & 21 \\
\hline 241-B-111 & Supernatant & 1.19 & 1.19 & 4 & $76.2 \%$ & $76.2 \%$ & 24 & 24 \\
\hline 241-B-111 & Solid & 1.27 & 1.19 & 910 & $63.1 \%$ & $76.2 \%$ & 24 & 24 \\
\hline $241-B-112$ & Supernatant & 1.51 & 1.51 & 11 & $46.2 \%$ & $46.2 \%$ & 20 & 20 \\
\hline $241-B-112$ & Solid & 1.49 & 1.51 & 121 & $41.0 \%$ & $46.2 \%$ & 20 & 20 \\
\hline $241-B-201$ & Supernatant & 0.00 & 0.00 & 0 & $0.0 \%$ & $0.0 \%$ & 18 & 18 \\
\hline 241-B-201 & Solid & 1.26 & 1.17 & 111 & $64.2 \%$ & $75.0 \%$ & 18 & 18 \\
\hline $241-\mathrm{B}-202$ & Supernatant & 0.00 & 0.00 & 0 & $0.0 \%$ & $0.0 \%$ & 18 & 18 \\
\hline 241-B-202 & Solid & 1.22 & 1.17 & 108 & $75.9 \%$ & $83.0 \%$ & 18 & 18 \\
\hline $241-B-203$ & Supernatant & 1.05 & 1.05 & 2 & $89.4 \%$ & $89.4 \%$ & 20 & 18 \\
\hline 241-B-203 & Solid & 1.19 & 1.05 & 188 & $75.7 \%$ & $89.4 \%$ & 20 & 18 \\
\hline 241-B-204 & Supernatant & 1.05 & 1.05 & 3 & $91.4 \%$ & $91.4 \%$ & 18 & 18 \\
\hline $241-\mathrm{B}-204$ & Solid & 1.19 & 1.05 & 184 & $77.3 \%$ & $91.4 \%$ & 18 & 18 \\
\hline $241-\mathrm{BX}-101$ & Supernatant & 0.00 & 0.00 & 0 & $0.0 \%$ & $0.0 \%$ & 22 & 22 \\
\hline $241-\mathrm{BX}-101$ & Solid & 1.68 & 1.28 & 180 & $15.5 \%$ & $75.0 \%$ & 22 & 22 \\
\hline $241-\mathrm{BX}-102$ & Supernatant & 0.00 & 0.00 & 0 & $0.0 \%$ & $0.0 \%$ & 22 & 22 \\
\hline $241-B X-102$ & Solid & 1.12 & 1.17 & 298 & $32.2 \%$ & $75.0 \%$ & 22 & 22 \\
\hline $241-B X-103$ & Supernatant & 1.07 & 1.07 & 50 & $77.8 \%$ & $77.8 \%$ & 23 & 23 \\
\hline $241-B X-103$ & Solid & 1.66 & 1.07 & 235 & $49.6 \%$ & $77.8 \%$ & 23 & 23 \\
\hline 241-BX-104 & Supernatant & 1.28 & 1.28 & 11 & $59.1 \%$ & $59.1 \%$ & 30 & 25 \\
\hline 241-BX-104 & Solid & 1.68 & 1.28 & 369 & $26.9 \%$ & $59.1 \%$ & 30 & 25 \\
\hline $241-B X-105$ & Supernatant & 1.29 & 1.29 & 18 & $68.7 \%$ & $68.7 \%$ & 20 & 20 \\
\hline 241-BX-105 & Solid & 1.69 & 1.29 & 254 & $13.8 \%$ & $68.7 \%$ & 20 & 20 \\
\hline 241-BX-106 & Supernatant & 0.00 & 0.00 & 0 & $46.2 \%$ & $46.2 \%$ & 20 & 20 \\
\hline 241-BX-106 & Solid & 1.62 & 1.51 & 143 & $39.9 \%$ & $46.2 \%$ & 20 & 20 \\
\hline $241-\mathrm{BX}-107$ & Supernatant & 0.00 & 0.00 & 0 & $0.0 \%$ & $0.0 \%$ & 20 & 20 \\
\hline $241-\mathrm{BX}-107$ & Solid & 1.44 & 1.17 & 1313 & $50.5 \%$ & $75.0 \%$ & 20 & 20 \\
\hline $241-\mathrm{BX}-108$ & Supernatant & 0.00 & 0.00 & 0 & $0.0 \%$ & $0.0 \%$ & 20 & 20 \\
\hline $241-B X-108$ & Solid & 1.46 & 1.17 & 119 & $17.2 \%$ & $75.0 \%$ & 20 & 20 \\
\hline 241-BX-109 & Supernatant & 0.00 & 0.00 & 0 & $0.0 \%$ & $0.0 \%$ & 22 & 22 \\
\hline 241-BX-109 & Solid & 1.52 & 1.17 & 730 & $50.6 \%$ & $72.0 \%$ & 22 & 22 \\
\hline 241-BX-110 & Supernatant & 1.44 & 1.44 & 5 & $53.1 \%$ & $53.1 \%$ & 20 & 20 \\
\hline $241-\mathrm{BX}-110$ & Solid & 1.67 & 1.44 & 807 & $41.7 \%$ & $53.1 \%$ & 20 & 20 \\
\hline 241-BX-111 & Supernatant & 0.00 & 0.00 & 0 & $51.2 \%$ & $51.2 \%$ & 19 & 18 \\
\hline 241-BX-111 & Solid & 1.45 & 1.45 & 713 & $24.8 \%$ & $51.2 \%$ & 19 & 18 \\
\hline
\end{tabular}


Table A-3. Tank Waste Physical Properties.

\begin{tabular}{|c|c|c|c|c|c|c|c|c|}
\hline Tank & Layer & $\begin{array}{l}\text { Bulk } \\
\text { SpG }\end{array}$ & $\begin{array}{c}\text { Liquid } \\
\text { SpG }\end{array}$ & \begin{tabular}{|c|} 
Non-RG \\
Waste Volume \\
$(\mathrm{kL})$
\end{tabular} & $\begin{array}{c}\text { Water in } \\
\text { Bulk } \\
\text { (wt\%) }\end{array}$ & $\begin{array}{l}\text { Water in } \\
\text { Liquid } \\
\text { (wt\%) }\end{array}$ & $\begin{array}{c}T_{\text {Waste }} \\
\left({ }^{\circ} \mathrm{C}\right)\end{array}$ & $\begin{array}{c}\mathbf{T}_{\text {Headspace }} \\
\left({ }^{\circ} \mathrm{C}\right)\end{array}$ \\
\hline $241-\mathrm{BX}-112$ & Supernatant & 1.18 & 1.18 & \begin{tabular}{|c|}
5 \\
\end{tabular} & $67.6 \%$ & $67.6 \%$ & 19 & 19 \\
\hline 241-BX-112 & Solid & 1.31 & 1.18 & 617 & $63.3 \%$ & $67.6 \%$ & 19 & 19 \\
\hline 241-BY-101 & Supernatant & 0.00 & 0.00 & 0 & $46.2 \%$ & $46.2 \%$ & 24 & 22 \\
\hline 241-BY-101 & Solid & 1.84 & 1.51 & 1400 & $22.3 \%$ & $46.2 \%$ & 24 & 22 \\
\hline 241-BY-102 & Supernatant & 0.00 & 0.00 & 0 & $50.8 \%$ & $50.8 \%$ & 0 & 22 \\
\hline 241-BY-102 & Solid & 1.57 & 1.46 & 1054 & $31.1 \%$ & $50.8 \%$ & 22 & 22 \\
\hline 241-BY-103 & Supernatant & 0.00 & 0.00 & 0 & $59.0 \%$ & $59.0 \%$ & 25 & 25 \\
\hline $241-\mathrm{BY}-103$ & Solid & 1.66 & 1.29 & 1564 & $27.8 \%$ & $59.0 \%$ & 25 & 25 \\
\hline 241-BY-104 & Supernatant & 0.00 & 0.00 & 0 & $48.9 \%$ & $48.9 \%$ & 44 & 25 \\
\hline 241-BY-104 & Solid & 1.71 & 1.51 & 1533 & $25.9 \%$ & $48.9 \%$ & 44 & 25 \\
\hline 241-BY-105 & Supernatant & 0.00 & 0.00 & 0 & $51.3 \%$ & $51.3 \%$ & 37 & 25 \\
\hline 241-BY-105 & Solid & 1.80 & 1.44 & 1821 & $17.9 \%$ & $51.3 \%$ & 37 & 25 \\
\hline 241-BY-106 & Supernatant & 0.00 & 0.00 & 0 & $59.4 \%$ & $59.4 \%$ & 40 & 33 \\
\hline 241-BY-106 & Solid & 1.65 & 1.31 & 1623 & $26.8 \%$ & $59.4 \%$ & 40 & 33 \\
\hline 241-BY-107 & Supernatant & 0.00 & 0.00 & 0 & $48.6 \%$ & $48.6 \%$ & 33 & 27 \\
\hline 241-BY-107 & Solid & 1.69 & 1.46 & 1028 & $37.8 \%$ & $48.6 \%$ & 33 & 27 \\
\hline 241-BY-108 & Supernatant & 0.00 & 0.00 & 0 & $32.7 \%$ & $32.7 \%$ & 37 & 25 \\
\hline 241-BY-108 & Solid & 1.48 & 1.33 & 841 & $27.3 \%$ & $32.7 \%$ & 37 & 25 \\
\hline 241-BY-109 & Supernatant & 0.00 & 0.00 & 0 & $53.0 \%$ & $53.0 \%$ & 0 & 17 \\
\hline 241-BY-109 & Solid & 1.71 & 1.50 & 1086 & $42.0 \%$ & $53.0 \%$ & 18 & 17 \\
\hline 241-BY-110 & Supernatant & 0.00 & 0.00 & 0 & $39.9 \%$ & $39.9 \%$ & 32 & 21 \\
\hline 241-BY-110 & Solid & 1.57 & 1.44 & 1384 & $28.3 \%$ & $39.9 \%$ & 32 & 21 \\
\hline 241-BY-111 & Supernatant & 0.00 & 0.00 & 0 & $53.8 \%$ & $53.8 \%$ & 26 & 24 \\
\hline 241-BY-111 & Solid & 1.67 & 1.42 & 1523 & $35.2 \%$ & $53.8 \%$ & 26 & 24 \\
\hline $241-\mathrm{BY}-112$ & Supernatant & 0.00 & 0.00 & 0 & $48.5 \%$ & $48.5 \%$ & 29 & 24 \\
\hline 241-BY-112 & Solid & 1.74 & 1.47 & 1083 & $29.6 \%$ & $48.5 \%$ & 29 & 24 \\
\hline $241-C-101$ & Supernatant & 0.00 & 0.00 & 0 & $0.0 \%$ & $0.0 \%$ & 32 & 32 \\
\hline $241-C-101$ & Solid & 1.78 & 1.17 & 333 & $23.4 \%$ & $74.0 \%$ & 32 & 32 \\
\hline $241-\mathrm{C}-102$ & Supernatant & 0.00 & 0.00 & $\mathbf{0}$ & $0.0 \%$ & $0.0 \%$ & 29 & 26 \\
\hline $241-\mathrm{C}-102$ & Solid & 1.68 & 1.17 & 1196 & $42.9 \%$ & $75.0 \%$ & 29 & 26 \\
\hline $241-C-103$ & Supernatant & 0.98 & 0.98 & 1 & $98.0 \%$ & $98.0 \%$ & 23 & 23 \\
\hline $241-C-103$ & Solid & 1.61 & 0.98 & 9 & $14.5 \%$ & $98.0 \%$ & 23 & 23 \\
\hline $241-C-104$ & Supernatant & 0.00 & 0.00 & 0 & $0.0 \%$ & $0.0 \%$ & 36 & 36 \\
\hline $241-C-104$ & Solid & 1.68 & 1.17 & 980 & $47.9 \%$ & $81.0 \%$ & 36 & 36 \\
\hline $241-C-105$ & Supernatant & 0.00 & 0.00 & 0 & $0.0 \%$ & $0.0 \%$ & 44 & 40 \\
\hline $241-\mathrm{C}-105$ & Solid & 1.55 & 1.23 & 500 & $28.3 \%$ & $67.0 \%$ & 44 & 40 \\
\hline $241-\mathrm{C}-106$ & Supernatant & 1.02 & 1.02 & 0 & $97.8 \%$ & $97.8 \%$ & 21 & 21 \\
\hline $241-\mathrm{C}-106$ & Solid & 1.56 & 1.02 & 10 & $41.9 \%$ & $97.8 \%$ & 21 & 21 \\
\hline $241-C-107$ & Supernatant & 0.00 & 0.00 & 0 & $0.0 \%$ & $0.0 \%$ & 43 & 39 \\
\hline $241-C-107$ & Solid & 1.55 & 1.17 & 935 & $47.5 \%$ & $79.0 \%$ & 43 & 39 \\
\hline $241-\mathrm{C}-108$ & Supernatant & 1.12 & 1.12 & 2 & $83.2 \%$ & $83.2 \%$ & 25 & 25 \\
\hline $241-C-108$ & Solid & 1.48 & 1.12 & 27 & $38.2 \%$ & $83.2 \%$ & 25 & 25 \\
\hline 241-C-109 & Supernatant & 0.00 & 0.00 & 0 & $0.0 \%$ & $0.0 \%$ & 23 & 23 \\
\hline $241-\mathrm{C}-109$ & Solid & 1.43 & 1.17 & 30 & $54.1 \%$ & $75.0 \%$ & 23 & 23 \\
\hline $241-\mathrm{C}-110$ & Supernatant & 0.00 & 0.00 & 0 & $82.9 \%$ & $82.9 \%$ & 22 & 22 \\
\hline $241-C-110$ & Solid & 1.44 & 1.10 & 97 & $60.2 \%$ & $82.9 \%$ & 22 & 22 \\
\hline $241-C-111$ & Supernatant & 0.00 & 0.00 & 0 & $0.0 \%$ & $0.0 \%$ & 28 & 24 \\
\hline $241-C-111$ & Solid & 1.55 & 1.17 & 217 & $36.1 \%$ & $75.0 \%$ & 28 & 24 \\
\hline $241-C-112$ & Supernatant & 0.00 & 0.00 & 0 & $0.0 \%$ & $0.0 \%$ & 28 & 28 \\
\hline $241-\mathrm{C}-112$ & Solid & 1.58 & 1.17 & 393 & $51.8 \%$ & $75.0 \%$ & 28 & 28 \\
\hline
\end{tabular}


Table A-3. Tank Waste Physical Properties.

\begin{tabular}{|c|c|c|c|c|c|c|c|c|}
\hline Tank & Layer & $\begin{array}{l}\text { Bulk } \\
\text { SpG }\end{array}$ & $\begin{array}{c}\text { Liquid } \\
\text { SpG }\end{array}$ & \begin{tabular}{|c|} 
Non-RG \\
Waste Volume \\
$(\mathrm{kL})$
\end{tabular} & $\begin{array}{c}\text { Water in } \\
\text { Bulk } \\
\text { (wt\%) }\end{array}$ & $\begin{array}{l}\text { Water in } \\
\text { Liquid } \\
\text { (wt\%) }\end{array}$ & $\begin{array}{l}T_{\text {Waste }} \\
\left({ }^{\circ} \mathbf{C}\right)\end{array}$ & $\begin{array}{c}\mathbf{T}_{\text {Headspace }} \\
\left({ }^{\circ} \mathrm{C}\right)\end{array}$ \\
\hline $241-\mathrm{C}-201$ & Supernatant & 1.00 & 1.00 & 0 & $100.0 \%$ & $100.0 \%$ & 16 & 20 \\
\hline 241-C-201 & Solid & 1.75 & 1.00 & 1 & $24.4 \%$ & $100.0 \%$ & 16 & 20 \\
\hline 241-C-202 & Supernatant & 1.00 & 1.00 & 0 & $100.0 \%$ & $100.0 \%$ & 16 & 19 \\
\hline $241-C-202$ & Solid & 1.75 & 1.00 & 1 & $24.4 \%$ & $100.0 \%$ & 16 & 19 \\
\hline $241-C-203$ & Supernatant & 1.00 & 1.00 & 0 & $100.0 \%$ & $100.0 \%$ & 19 & 19 \\
\hline $241-C-203$ & Solid & 1.93 & 1.00 & 0 & $35.5 \%$ & $100.0 \%$ & 15 & 19 \\
\hline 241-C-204 & Supernatant & 1.00 & 1.00 & 0 & $100.0 \%$ & $100.0 \%$ & 17 & 16 \\
\hline $241-C-204$ & Solid & 1.77 & 1.00 & 1 & $38.2 \%$ & $100.0 \%$ & 17 & 16 \\
\hline $241-S-101$ & Supernatant & 0.00 & 0.00 & 0 & $50.7 \%$ & $50.7 \%$ & 37 & 29 \\
\hline $241-S-101$ & Solid & 1.65 & 1.47 & 1332 & $39.2 \%$ & $50.7 \%$ & 37 & 29 \\
\hline $241-\mathrm{S}-102$ & Supernatant & 0.00 & 0.00 & 0 & $0.0 \%$ & $0.0 \%$ & 0 & 25 \\
\hline $241-S-102$ & Solid & 1.53 & 1.46 & 113 & $28.0 \%$ & $48.5 \%$ & 28 & 25 \\
\hline $241-S-103$ & Supernatant & 1.45 & 1.45 & 4 & $49.8 \%$ & $49.8 \%$ & 27 & 23 \\
\hline $241-S-103$ & Solid & 1.61 & 1.45 & 892 & $35.7 \%$ & $49.8 \%$ & 27 & 23 \\
\hline $241-S-104$ & Supernatant & 0.00 & 0.00 & 0 & $55.1 \%$ & $55.1 \%$ & 37 & 29 \\
\hline $241-S-104$ & Solid & 1.67 & 1.37 & 1090 & $38.8 \%$ & $55.1 \%$ & 37 & 29 \\
\hline $241-\mathrm{S}-105$ & Supernatant & 0.00 & 0.00 & 0 & $49.1 \%$ & $49.1 \%$ & 25 & 23 \\
\hline 241-S-105 & Solid & 1.66 & 1.45 & 1536 & $7.7 \%$ & $49.1 \%$ & 25 & 23 \\
\hline $241-S-106$ & Supernatant & 0.00 & 0.00 & 0 & $54.2 \%$ & $54.2 \%$ & 24 & 24 \\
\hline $241-S-106$ & Solid & 1.72 & 1.43 & 1723 & $34.5 \%$ & $54.2 \%$ & 24 & 24 \\
\hline 241-S-107 & Supernatant & 0.00 & 0.00 & 0 & $0.0 \%$ & $0.0 \%$ & 34 & 28 \\
\hline $241-S-107$ & Solid & 1.78 & 1.31 & 1356 & $34.1 \%$ & $64.0 \%$ & 34 & 28 \\
\hline $241-S-108$ & Supernatant & 0.00 & 0.00 & 0 & $49.1 \%$ & $49.1 \%$ & 28 & 24 \\
\hline 241-S-108 & Solid & 1.68 & 1.45 & 2082 & $30.4 \%$ & $49.1 \%$ & 28 & 24 \\
\hline 241-S-109 & Supernatant & 0.00 & 0.00 & 0 & $53.1 \%$ & $53.1 \%$ & 26 & 23 \\
\hline $241-S-109$ & Solid & 1.66 & 1.49 & 2017 & $10.7 \%$ & $53.1 \%$ & 26 & 23 \\
\hline $241-S-110$ & Supernatant & 0.00 & 0.00 & 0 & $49.3 \%$ & $49.3 \%$ & 42 & 26 \\
\hline $241-S-110$ & Solid & 1.23 & 1.43 & 1473 & $20.0 \%$ & $49.3 \%$ & 42 & 26 \\
\hline $241-S-111$ & Supernatant & 0.00 & 0.00 & 0 & $51.0 \%$ & $51.0 \%$ & 28 & 26 \\
\hline $241-S-111$ & Solid & 1.43 & 1.45 & 1517 & $29.0 \%$ & $51.0 \%$ & 28 & 26 \\
\hline $241-S-112$ & Supernatant & 1.27 & 1.27 & 0 & $73.3 \%$ & $73.3 \%$ & 21 & 21 \\
\hline $241-S-112$ & Solid & 1.90 & 1.27 & 9 & $22.8 \%$ & $73.3 \%$ & 21 & 21 \\
\hline $241-\mathrm{SX}-101$ & Supernatant & 0.00 & 0.00 & 0 & $49.1 \%$ & $49.1 \%$ & 51 & 37 \\
\hline 241-SX-101 & Solid & 1.68 & 1.48 & 1584 & $26.6 \%$ & $49.1 \%$ & 51 & 37 \\
\hline $241-\mathrm{SX}-102$ & Supernatant & 0.00 & 0.00 & 0 & $48.1 \%$ & $48.1 \%$ & 53 & 35 \\
\hline 241-SX-102 & Solid & 1.70 & 1.46 & 1292 & $39.3 \%$ & $48.1 \%$ & 53 & 35 \\
\hline 241-SX-103 & Supernatant & 0.00 & 0.00 & 0 & $46.6 \%$ & $46.6 \%$ & 60 & 31 \\
\hline 241-SX-103 & Solid & 1.73 & 1.47 & 1926 & $30.5 \%$ & $46.6 \%$ & 60 & 31 \\
\hline 241-SX-104 & Supernatant & 0.00 & 0.00 & 0 & $45.3 \%$ & $45.3 \%$ & 57 & 35 \\
\hline 241-SX-104 & Solid & 1.69 & 1.47 & 1688 & $28.2 \%$ & $45.3 \%$ & 57 & 35 \\
\hline $241-\mathrm{SX}-105$ & Supernatant & 0.00 & 0.00 & 0 & $42.9 \%$ & $42.9 \%$ & 60 & 33 \\
\hline 241-SX-105 & Solid & 1.63 & 1.47 & 1422 & $35.1 \%$ & $42.9 \%$ & 60 & 33 \\
\hline 241-SX-106 & Supernatant & 0.00 & 0.00 & 0 & $49.4 \%$ & $49.4 \%$ & 33 & 31 \\
\hline $241-S X-106$ & Solid & 1.58 & 1.29 & 1501 & $39.8 \%$ & $49.4 \%$ & 33 & 31 \\
\hline 241-SX-107 & Supernatant & 0.00 & 0.00 & 0 & $0.0 \%$ & $0.0 \%$ & 74 & 52 \\
\hline 241-SX-107 & Solid & 1.77 & 1.50 & 356 & $22.7 \%$ & $51.9 \%$ & 74 & 52 \\
\hline 241-SX-108 & Supernatant & 0.00 & 0.00 & 0 & $0.0 \%$ & $0.0 \%$ & 81 & 59 \\
\hline 241-SX-108 & Solid & 1.77 & 1.17 & 280 & $2.0 \%$ & $75.0 \%$ & 81 & 59 \\
\hline 241-SX-109 & Supernatant & 0.00 & 0.00 & 0 & $0.0 \%$ & $0.0 \%$ & 58 & 50 \\
\hline 241-SX-109 & Solid & 1.73 & 1.39 & 913 & $23.2 \%$ & $51.0 \%$ & 58 & 50 \\
\hline
\end{tabular}


Table A-3. Tank Waste Physical Properties.

\begin{tabular}{|c|c|c|c|c|c|c|c|c|}
\hline Tank & Layer & $\begin{array}{l}\text { Bulk } \\
\text { SpG }\end{array}$ & $\begin{array}{l}\text { Liquid } \\
\text { SpG }\end{array}$ & $\begin{array}{c}\text { Non-RG } \\
\text { Waste Volume } \\
\text { (kL) }\end{array}$ & $\begin{array}{c}\text { Water in } \\
\text { Bulk } \\
\text { (wt\%) }\end{array}$ & $\begin{array}{l}\text { Water in } \\
\text { Liquid } \\
\text { (wt\%) }\end{array}$ & $\begin{array}{c}T_{\text {Waste }} \\
\left({ }^{\circ} \mathbf{C}\right)\end{array}$ & $\begin{array}{c}\mathbf{T}_{\text {Headspace }} \\
\left({ }^{\circ} \mathbf{C}\right)\end{array}$ \\
\hline 241-SX-110 & Supernatant & 0.00 & 0.00 & 0 & $0.0 \%$ & $0.0 \%$ & 73 & 50 \\
\hline 241-SX-110 & Solid & 1.76 & 1.17 & 212 & $22.8 \%$ & $75.0 \%$ & 73 & 50 \\
\hline $241-S X-111$ & Supernatant & 0.00 & 0.00 & 0 & $0.0 \%$ & $0.0 \%$ & 82 & 59 \\
\hline $241-S X-111$ & Solid & 1.76 & 1.50 & 436 & $22.9 \%$ & $51.9 \%$ & 82 & 59 \\
\hline $241-\mathrm{SX}-112$ & Supernatant & 0.00 & 0.00 & 0 & $0.0 \%$ & $0.0 \%$ & 63 & 52 \\
\hline $241-\mathrm{SX}-112$ & Solid & 1.77 & 1.50 & 283 & $22.7 \%$ & $51.9 \%$ & 63 & 52 \\
\hline $241-S X-113$ & Supernatant & 0.00 & 0.00 & 0 & $0.0 \%$ & $0.0 \%$ & 29 & 29 \\
\hline 241-SX-113 & Solid & 1.43 & 1.17 & 72 & $46.0 \%$ & $75.0 \%$ & 29 & 29 \\
\hline $241-S X-114$ & Supernatant & 0.00 & 0.00 & 0 & $51.9 \%$ & $51.9 \%$ & 78 & 58 \\
\hline $241-S X-114$ & Solid & 1.75 & 1.50 & 588 & $23.9 \%$ & $51.9 \%$ & 78 & 58 \\
\hline 241-SX-115 & Supernatant & 0.00 & 0.00 & 0 & $0.0 \%$ & $0.0 \%$ & 0 & 25 \\
\hline 241-SX-115 & Solid & 1.77 & 1.17 & 16 & $10.1 \%$ & $75.0 \%$ & 25 & 25 \\
\hline $241-\mathrm{T}-101$ & Supernatant & 0.00 & 0.00 & 0 & $49.1 \%$ & $49.1 \%$ & 22 & 21 \\
\hline 241-T-101 & Solid & 1.54 & 1.45 & 376 & $39.5 \%$ & $49.1 \%$ & 22 & 21 \\
\hline 241-T-102 & Supernatant & 1.14 & 1.14 & 48 & $79.8 \%$ & $79.8 \%$ & 18 & 18 \\
\hline $241-\mathrm{T}-102$ & Solid & 1.80 & 1.14 & 72 & $29.6 \%$ & $79.8 \%$ & 18 & 18 \\
\hline $241-T-103$ & Supernatant & 1.19 & 1.19 & 15 & $71.6 \%$ & $71.6 \%$ & 21 & 21 \\
\hline $241-\mathrm{T}-103$ & Solid & 1.71 & 1.19 & 87 & $37.2 \%$ & $71.6 \%$ & 21 & 21 \\
\hline $241-T-104$ & Supernatant & 0.00 & 0.00 & 0 & $0.0 \%$ & $0.0 \%$ & 20 & 20 \\
\hline $241-T-104$ & Solid & 1.29 & 1.17 & 1199 & $70.5 \%$ & $84.0 \%$ & 20 & 20 \\
\hline $241-\mathrm{T}-105$ & Supernatant & 0.00 & 0.00 & 0 & $0.0 \%$ & $0.0 \%$ & 0 & 16 \\
\hline $241-\mathrm{T}-105$ & Solid & 1.46 & 1.18 & 371 & $53.0 \%$ & $80.0 \%$ & 17 & 16 \\
\hline $241-T-106$ & Supernatant & 0.00 & 0.00 & 0 & $0.0 \%$ & $0.0 \%$ & 20 & 20 \\
\hline $241-T-106$ & Solid & 1.59 & 1.17 & 82 & $16.7 \%$ & $75.0 \%$ & 20 & 20 \\
\hline $241-\mathrm{T}-107$ & Supernatant & 0.00 & 0.00 & 0 & $0.0 \%$ & $0.0 \%$ & 28 & 20 \\
\hline $241-T-107$ & Solid & 1.56 & 1.17 & 655 & $45.7 \%$ & $84.0 \%$ & 28 & 20 \\
\hline $241-T-108$ & Supernatant & 0.00 & 0.00 & 0 & $69.6 \%$ & $69.6 \%$ & 19 & 19 \\
\hline $241-T-108$ & Solid & 1.55 & 1.26 & 60 & $34.5 \%$ & $69.6 \%$ & 19 & 19 \\
\hline 241-T-109 & Supernatant & 0.00 & 0.00 & 0 & $69.6 \%$ & $69.6 \%$ & 19 & 19 \\
\hline $241-\mathrm{T}-109$ & Solid & 1.65 & 1.26 & 235 & $48.7 \%$ & $69.6 \%$ & 19 & 19 \\
\hline $241-\mathrm{T}-110$ & Supernatant & 1.05 & 1.05 & 3 & $86.8 \%$ & $86.8 \%$ & 22 & 22 \\
\hline $241-\mathrm{T}-110$ & Solid & 1.25 & 1.05 & 1397 & $75.5 \%$ & $86.8 \%$ & 22 & 22 \\
\hline 241-T-111 & Supernatant & 0.00 & 0.00 & 0 & $0.0 \%$ & $0.0 \%$ & 20 & 20 \\
\hline $241-\mathrm{T}-111$ & Solid & 0.00 & 1.17 & 1691 & $75.3 \%$ & $75.0 \%$ & 20 & 20 \\
\hline $241-T-112$ & Supernatant & 1.10 & 1.10 & 26 & $85.1 \%$ & $85.1 \%$ & 20 & 20 \\
\hline $241-T-112$ & Solid & 1.28 & 1.10 & 226 & $73.9 \%$ & $85.1 \%$ & 20 & 20 \\
\hline $241-\mathrm{T}-201$ & Supernatant & 1.06 & 1.06 & 8 & $91.2 \%$ & $91.2 \%$ & 19 & 19 \\
\hline $241-\mathrm{T}-201$ & Solid & 1.31 & 1.06 & 107 & $67.5 \%$ & $91.2 \%$ & 19 & 19 \\
\hline 241-T-202 & Supernatant & 0.00 & 0.00 & 0 & $0.0 \%$ & $0.0 \%$ & 20 & 19 \\
\hline 241-T-202 & Solid & 1.18 & 1.17 & 77 & $75.8 \%$ & $75.0 \%$ & 20 & 19 \\
\hline 241-T-203 & Supernatant & 0.00 & 0.00 & 0 & $0.0 \%$ & $0.0 \%$ & 19 & 19 \\
\hline 241-T-203 & Solid & 1.22 & 1.17 & 136 & $76.2 \%$ & $85.0 \%$ & 19 & 19 \\
\hline $241-T-204$ & Supernatant & 0.00 & 0.00 & 0 & $0.0 \%$ & $0.0 \%$ & 20 & 19 \\
\hline 241-T-204 & Solid & 1.18 & 1.17 & 136 & $75.0 \%$ & $75.0 \%$ & 20 & 19 \\
\hline $241-T X-101$ & Supernatant & 0.00 & 0.00 & 0 & $49.1 \%$ & $49.1 \%$ & 0 & 23 \\
\hline 241-TX-101 & Solid & 1.74 & 1.45 & 344 & $25.1 \%$ & $49.1 \%$ & 25 & 23 \\
\hline 241-TX-102 & Supernatant & 0.00 & 0.00 & 0 & $49.1 \%$ & $49.1 \%$ & 27 & 26 \\
\hline 241-TX-102 & Solid & 1.61 & 1.45 & 821 & $31.2 \%$ & $49.1 \%$ & 27 & 26 \\
\hline 241-TX-103 & Supernatant & 0.00 & 0.00 & 0 & $49.7 \%$ & $49.7 \%$ & 24 & 21 \\
\hline 241-TX-103 & Solid & 1.61 & 1.44 & 548 & $31.2 \%$ & $49.7 \%$ & 24 & 21 \\
\hline
\end{tabular}


Table A-3. Tank Waste Physical Properties.

\begin{tabular}{|c|c|c|c|c|c|c|c|c|}
\hline Tank & Layer & $\begin{array}{l}\text { Bulk } \\
\text { SpG }\end{array}$ & $\begin{array}{l}\text { Liquid } \\
\text { SpG }\end{array}$ & $\begin{array}{c}\text { Non-RG } \\
\text { Waste Volume } \\
(\mathbf{k L}) \\
\end{array}$ & $\begin{array}{c}\text { Water in } \\
\text { Bulk } \\
\text { (wt\%) } \\
\end{array}$ & $\begin{array}{l}\text { Water in } \\
\text { Liquid } \\
\text { (wt\%) }\end{array}$ & $\begin{array}{l}T_{\text {Waste }} \\
\left({ }^{\circ} \mathbf{C}\right)\end{array}$ & $\begin{array}{c}\mathbf{T}_{\text {Headspace }} \\
\left({ }^{\circ} \mathbf{C}\right)\end{array}$ \\
\hline $241-\mathrm{TX}-104$ & Supernatant & 1.44 & 1.44 & 9 & $51.9 \%$ & $51.9 \%$ & 22 & 22 \\
\hline $241-T X-104$ & Solid & 1.74 & 1.44 & 253 & $45.5 \%$ & $51.9 \%$ & 22 & 22 \\
\hline 241-TX-105 & Supernatant & 0.00 & 0.00 & 0 & $49.1 \%$ & $49.1 \%$ & 34 & 34 \\
\hline 241-TX-105 & Solid & 1.63 & 1.45 & 2181 & $29.4 \%$ & $49.1 \%$ & 34 & 34 \\
\hline $241-\mathrm{TX}-106$ & Supernatant & 0.00 & 0.00 & 0 & $49.1 \%$ & $49.1 \%$ & 26 & 26 \\
\hline $241-T X-106$ & Solid & 1.62 & 1.45 & 1318 & $30.4 \%$ & $49.1 \%$ & 26 & 26 \\
\hline 241-TX-107 & Supernatant & 0.00 & 0.00 & 0 & $49.9 \%$ & $49.9 \%$ & 19 & 19 \\
\hline $241-\mathrm{TX}-107$ & Solid & 1.78 & 1.46 & 112 & $27.7 \%$ & $49.9 \%$ & 19 & 19 \\
\hline 241-TX-108 & Supernatant & 0.00 & 0.00 & 0 & $49.1 \%$ & $49.1 \%$ & 21 & 21 \\
\hline 241-TX-108 & Solid & 1.62 & 1.45 & 478 & $30.7 \%$ & $49.1 \%$ & 21 & 21 \\
\hline 241-TX-109 & Supernatant & 0.00 & 0.00 & 0 & $0.0 \%$ & $0.0 \%$ & 41 & 41 \\
\hline 241-TX-109 & Solid & 1.43 & 1.17 & 1375 & $54.1 \%$ & $75.0 \%$ & 41 & 41 \\
\hline $241-\mathrm{TX}-110$ & Supernatant & 0.00 & 0.00 & 0 & $49.1 \%$ & $49.1 \%$ & 0 & 22 \\
\hline $241-\mathrm{TX}-110$ & Solid & 1.62 & 1.45 & 1769 & $30.6 \%$ & $49 . \overline{1 \%}$ & 28 & 22 \\
\hline 241-TX-111 & Supernatant & 0.00 & 0.00 & 0 & $49.1 \%$ & $49.1 \%$ & 27 & 25 \\
\hline 241-TX-111 & Solid & 1.61 & 1.45 & 1381 & $31.3 \%$ & $49.1 \%$ & 27 & 25 \\
\hline 241-TX-112 & Supernatant & 0.00 & 0.00 & 0 & $51.6 \%$ & $51.6 \%$ & 25 & 24 \\
\hline $241-\mathrm{TX}-112$ & Solid & 1.63 & 1.42 & 2398 & $29.1 \%$ & $51.6 \%$ & 25 & 24 \\
\hline $241-\mathrm{TX}-113$ & Supernatant & 0.00 & 0.00 & 0 & $49.1 \%$ & $49.1 \%$ & 24 & 22 \\
\hline $241-\mathrm{TX}-113$ & Solid & 1.61 & 1.45 & 2416 & $15.5 \%$ & $49.1 \%$ & 24 & 22 \\
\hline 241-TX-114 & Supernatant & 0.00 & 0.00 & 0 & $61.2 \%$ & $61.2 \%$ & 0 & 18 \\
\hline $241-\mathrm{TX}-114$ & Solid & 1.63 & 1.33 & 2012 & $29.0 \%$ & $61.2 \%$ & 21 & 18 \\
\hline $241-\mathrm{TX}-115$ & Supernatant & 0.00 & 0.00 & 0 & $49.1 \%$ & $49.1 \%$ & 23 & 22 \\
\hline $241-\mathrm{TX}-115$ & Solid & 1.63 & 1.45 & 2093 & $29.5 \%$ & $49.1 \%$ & 23 & 22 \\
\hline $241-\mathrm{TX}-116$ & Supernatant & 0.00 & 0.00 & 0 & $53.0 \%$ & $53.0 \%$ & 0 & 20 \\
\hline $241-\mathrm{TX}-116$ & Solid & 1.66 & 1.39 & 2264 & $21.2 \%$ & $53.0 \%$ & 21 & 20 \\
\hline $241-\mathrm{TX}-117$ & Supernatant & 0.00 & 0.00 & 0 & $69.6 \%$ & $69.6 \%$ & 0 & 20 \\
\hline 241-TX-117 & Solid & 1.58 & 1.26 & 1817 & $27.0 \%$ & $69.6 \%$ & 21 & 20 \\
\hline 241-TX-118 & Supernatant & 0.00 & 0.00 & 0 & $49.1 \%$ & $49.1 \%$ & 25 & 24 \\
\hline 241-TX-118 & Solid & 1.69 & 1.45 & 935 & $44.7 \%$ & $49.1 \%$ & 25 & 24 \\
\hline 241-TY-101 & Supernatant & 0.00 & 0.00 & 0 & $69.6 \%$ & $69.6 \%$ & 21 & 21 \\
\hline 241-TY-101 & Solid & 1.63 & 1.26 & 447 & $44.2 \%$ & $69.6 \%$ & 21 & 21 \\
\hline 241-TY-102 & Supernatant & 0.00 & 0.00 & 0 & $57.8 \%$ & $57.8 \%$ & 19 & 19 \\
\hline 241-TY-102 & Solid & 1.76 & 1.36 & 262 & $32.0 \%$ & $57.8 \%$ & 19 & 19 \\
\hline 241-TY-103 & Supernatant & 0.00 & 0.00 & 0 & $49.1 \%$ & $49.1 \%$ & 24 & 24 \\
\hline 241-TY-103 & Solid & 1.68 & 1.45 & 585 & $51.7 \%$ & $49.1 \%$ & 24 & 24 \\
\hline 241-TY-104 & Supernatant & 1.18 & 1.18 & 5 & $77.5 \%$ & $77.5 \%$ & 23 & 23 \\
\hline 241-TY-104 & Solid & 1.65 & 1.18 & 163 & $51.9 \%$ & $77.5 \%$ & 23 & 23 \\
\hline 241-TY-105 & Supernatant & 0.00 & 0.00 & 0 & $0.0 \%$ & $0.0 \%$ & 25 & 24 \\
\hline 241-TY-105 & Solid & 1.53 & 1.17 & 874 & $39.4 \%$ & $75.0 \%$ & 25 & 24 \\
\hline 241-TY-106 & Supernatant & 0.00 & 0.00 & 0 & $0.0 \%$ & $0.0 \%$ & 20 & 20 \\
\hline $241-\mathrm{TY}-107$ & Solid & 1.40 & 1.17 & 62 & $38.1 \%$ & $75.0 \%$ & 20 & 20 \\
\hline $241-U-101$ & Supernatant & 0.00 & 0.00 & 0 & $0.0 \%$ & $0.0 \%$ & 21 & 20 \\
\hline 241-U-101 & Solid & 1.77 & 1.17 & 87 & $29.8 \%$ & $76.0 \%$ & 21 & 20 \\
\hline 241-U-102 & Supernatant & 1.48 & 1.48 & 4 & $47.5 \%$ & $47.5 \%$ & 26 & 24 \\
\hline $241-\mathrm{U}-102$ & Solid & 1.67 & 1.48 & 1234 & $34.1 \%$ & $47.5 \%$ & 26 & 24 \\
\hline 241-U-103 & Supernatant & 1.44 & 1.44 & 2 & $49.9 \%$ & $49.9 \%$ & 27 & 26 \\
\hline 241-U-103 & Solid & 1.52 & 1.44 & 1576 & $39.5 \%$ & $49.9 \%$ & 27 & 26 \\
\hline $241-U-104$ & Supernatant & 0.00 & 0.00 & 0 & $0.0 \%$ & $0.0 \%$ & 0 & 24 \\
\hline $241-U-104$ & Solid & 1.12 & 1.40 & 205 & $71.4 \%$ & $55.0 \%$ & 28 & 24 \\
\hline
\end{tabular}


Table A-3. Tank Waste Physical Properties.

\begin{tabular}{|c|c|c|c|c|c|c|c|c|}
\hline Tank & Layer & $\begin{array}{c}\text { Bulk } \\
\text { SpG }\end{array}$ & $\begin{array}{c}\text { Liquid } \\
\text { SpG }\end{array}$ & $\begin{array}{c}\text { Non-RG } \\
\text { Waste Volume } \\
(\mathbf{k L})\end{array}$ & $\begin{array}{c}\text { Water in } \\
\text { Bulk } \\
(\mathbf{w t} \%)\end{array}$ & $\begin{array}{c}\text { Water in } \\
\text { Liquid } \\
(\mathbf{w t} \%)\end{array}$ & $\begin{array}{c}\mathbf{T}_{\text {Waste }} \\
\left({ }^{\circ} \mathbf{C}\right)\end{array}$ & $\begin{array}{c}\mathbf{T}_{\text {Headspace }} \\
\left({ }^{\circ} \mathbf{C}\right)\end{array}$ \\
\hline $241-U-105$ & Supernatant & 0.00 & 0.00 & $\mathbf{0}$ & $51.6 \%$ & $30.4 \%$ & 31 & 25 \\
\hline $241-U-105$ & Solid & 1.67 & 1.46 & 1336 & $30.4 \%$ & $30.4 \%$ & 31 & 25 \\
\hline $241-U-106$ & Supernatant & 1.34 & 1.34 & 6 & $48.6 \%$ & $48.6 \%$ & 26 & 24 \\
\hline $241-U-106$ & Solid & 1.55 & 1.34 & 638 & $41.6 \%$ & $48.6 \%$ & 26 & 24 \\
\hline $241-U-107$ & Supernatant & 0.00 & 0.00 & 0 & $49.6 \%$ & $49.6 \%$ & 23 & 22 \\
\hline $241-U-107$ & Solid & 1.74 & 1.43 & 1113 & $32.6 \%$ & $49.6 \%$ & 23 & 22 \\
\hline $241-U-108$ & Supernatant & 0.00 & 0.00 & 0 & $49.9 \%$ & $49.9 \%$ & 27 & 24 \\
\hline $241-U-108$ & Solid & 1.68 & 1.40 & 1641 & $36.9 \%$ & $49.9 \%$ & 27 & 24 \\
\hline $241-U-109$ & Supernatant & 0.00 & 0.00 & 0 & $50.7 \%$ & $50.7 \%$ & 27 & 26 \\
\hline $241-U-109$ & Solid & 1.47 & 1.47 & 1518 & $28.8 \%$ & $50.7 \%$ & 27 & 26 \\
\hline $241-U-110$ & Supernatant & 0.00 & 0.00 & 0 & $0.0 \%$ & $0.0 \%$ & 25 & 24 \\
\hline $241-U-110$ & Solid & 1.72 & 1.17 & 665 & $30.5 \%$ & $75.0 \%$ & 25 & 24 \\
\hline $241-U-111$ & Supernatant & 0.00 & 0.00 & 0 & $52.6 \%$ & $52.6 \%$ & 25 & 23 \\
\hline $241-U-111$ & Solid & 1.61 & 1.40 & 840 & $35.9 \%$ & $52.6 \%$ & 25 & 23 \\
\hline $241-U-112$ & Supernatant & 0.00 & 0.00 & 0 & $0.0 \%$ & $0.0 \%$ & 21 & 21 \\
\hline $241-U-112$ & Solid & 1.74 & 1.17 & 172 & $32.3 \%$ & $75.0 \%$ & 21 & 21 \\
\hline $241-U-201$ & Supernatant & 1.26 & 1.26 & 4 & $69.3 \%$ & $69.3 \%$ & 11 & 10 \\
\hline $241-U-201$ & Solid & 1.63 & 1.26 & 11 & $27.1 \%$ & $69.3 \%$ & 11 & 10 \\
\hline $241-U-202$ & Supernatant & 1.28 & 1.28 & 4 & $69.1 \%$ & $69.1 \%$ & 20 & 20 \\
\hline $241-U-202$ & Solid & 1.51 & 1.28 & 10 & $27.8 \%$ & $69.1 \%$ & 20 & 20 \\
\hline $241-U-203$ & Supernatant & 1.28 & 1.28 & 4 & $68.7 \%$ & $68.7 \%$ & 21 & 21 \\
\hline $241-U-203$ & Solid & 1.59 & 1.28 & 9 & $40.2 \%$ & $68.7 \%$ & 21 & 21 \\
\hline $241-U-204$ & Supernatant & 1.11 & 1.11 & 4 & $87.0 \%$ & $87.0 \%$ & 19 & 19 \\
\hline $241-U-204$ & Solid & 1.47 & 1.11 & 7 & $26.2 \%$ & $87.0 \%$ & 19 & 19 \\
\hline
\end{tabular}


RPP-5926 REV 9

APPENDIX B

CALCULATIONS OF THE GAS DIFFUSION COEFFICIENTS FOR THE CONCRETE DOME OF SINGLE SHELL TANKS 


\section{B1.0 APPROACH}

Hydrogen (and other decomposition gases) would begin to build in concentration in the headspace under zero ventilation. Headspace concentration would increase until the system reached steady state and the diffusion outflow equaled the gas generation rate.

The approach used to estimate hydrogen diffusion through a single-shell tank dome is documented in RPP-18491 and is summarized as follows:

1. Estimate the effective diffusivity for hydrogen through concrete, asphaltic membrane, mortar/gunite, and soil (as appropriate).

2. Calculate a diffusion resistance term for each of the barriers listed in 1 above.

3. Sum the resistances for the respective diffusion barriers.

4. Calculate the amount of hydrogen that could diffuse through the dome.

\section{B2.0 DOME DIFFUSION INPUTS}

Table B-1 discusses diffusion barriers and coefficients taken from RPP-18491. Table B-2 shows diffusion resistance and diffusion rates through all 149 single-shell tank domes.

\section{B3.0 REFERENCES}

RPP-18491, 2003, Flammable Gas Diffusion Through Single-Shell Tank Domes, Rev. 0, CH2M HILL Hanford Group, Inc., Richland, Washington. 
Table B-1. The Thickness of Dome Coatings for 149 Single-Shell Tanks and Diffusion Coefficients for Hydrogen and Methane.

\begin{tabular}{|c|c|c|c|c|c|c|c|c|c|c|c|c|c|c|}
\hline Tank & $\begin{array}{c}\text { Dome } \\
\text { Area } \\
\left(\mathrm{cm}^{2}\right) \\
\end{array}$ & Tank Dome Coatings & $\begin{array}{c}\text { Concrete } \\
\text { Thick } \\
\text { (cm) } \\
\end{array}$ & $\begin{array}{c}\text { Asphaltic } \\
\text { Thick } \\
\text { (cm) }\end{array}$ & $\begin{array}{l}\text { Gunite } \\
\text { Thick } \\
\text { (cm) }\end{array}$ & $\begin{array}{l}\text { Soil } \\
\text { Thick } \\
\text { (cm) }\end{array}$ & $\begin{array}{l}\text { Concrete } \\
\text { De of } \mathrm{H}_{2} \\
\left(\mathrm{~cm}^{2} / \mathrm{sec}\right)\end{array}$ & $\begin{array}{l}\text { Asphaltic } \\
\text { De of } \mathrm{H}_{2} \\
\left(\mathrm{~cm}^{2} / \mathrm{sec}\right)\end{array}$ & $\begin{array}{c}\text { Gunite } \\
\text { De of } \mathrm{H}_{2} \\
\left(\mathrm{~cm}^{2} / \mathrm{sec}\right)\end{array}$ & $\begin{array}{c}\text { Soil } \\
\text { De of } \mathrm{H}_{2} \\
\left(\mathrm{~cm}^{2} / \mathrm{sec}\right)\end{array}$ & $\begin{array}{c}\text { Concrete De } \\
\text { of CH} \\
\left(\mathrm{cm}^{2} / \mathrm{sec}\right)\end{array}$ & $\begin{array}{c}\text { Asphaltic } \\
\text { De of CH4 } \\
\left(\mathrm{cm}^{2} / \mathrm{sec}\right) \\
\end{array}$ & $\begin{array}{c}\text { Gunite De } \\
\text { of CH} \\
\left(\mathrm{cm}^{2} / \mathrm{sec}\right)\end{array}$ & $\begin{array}{c}\text { Soil } \\
\mathrm{De}^{\text {of } \mathrm{CH}_{4}} \\
\left(\mathrm{~cm}^{2} / \mathrm{sec}\right)\end{array}$ \\
\hline A-101 & $4.39 \mathrm{E}+06$ & 2-Ply & 38.1 & 0.6350 & 0.000 & 366 & $3.80 \mathrm{E}-03$ & $5.00 \mathrm{E}-07$ & $3.80 \mathrm{E}-03$ & 0.12 & $1.20 \mathrm{E}-03$ & $5.00 \mathrm{E}-07$ & $1.20 \mathrm{E}-03$ & 0.037 \\
\hline$A-102$ & $4.39 \mathrm{E}+06$ & 2-Ply & 38.1 & 0.6350 & 0.000 & 366 & $3.80 \mathrm{E}-03$ & \begin{tabular}{|l|}
$5.00 \mathrm{E}-07$ \\
\end{tabular} & $3.80 \mathrm{E}-03$ & 0.12 & $1.20 \mathrm{E}-03$ & $5.00 \mathrm{E}-07$ & $1.20 \mathrm{E}-03$ & 0.037 \\
\hline A-103 & $4.39 \mathrm{E}+06$ & 2-Ply & 38.1 & 0.6350 & 0.000 & 366 & $3.80 \mathrm{E}-03$ & $5.00 \mathrm{E}-07$ & $3.80 \mathrm{E}-03$ & 0.12 & $1.20 \mathrm{E}-03$ & $5.00 \mathrm{E}-07$ & $1.20 \mathrm{E}-03$ & 0.037 \\
\hline A-104 & $4.39 \mathrm{E}+06$ & 2-Ply & 38.1 & 0.6350 & 0.000 & 366 & $3.80 \mathrm{E}-03$ & $5.00 \mathrm{E}-07$ & $3.80 \mathrm{E}-03$ & 0.12 & $1.20 \mathrm{E}-03$ & $5.00 \mathrm{E}-07$ & $1.20 \mathrm{E}-03$ & 0.037 \\
\hline A-105 & $4.39 \mathrm{E}+06$ & 2-Ply & 38.1 & 0.6350 & 0.000 & 366 & $3.80 \mathrm{E}-03$ & $5.00 \mathrm{E}-07$ & $3.80 \mathrm{E}-03$ & 0.12 & $1.20 \mathrm{E}-03$ & $5.00 \mathrm{E}-07$ & $1.20 \mathrm{E}-03$ & 0.037 \\
\hline A-106 & $4.39 \mathrm{E}+06$ & 2-Ply & 38.1 & 0.6350 & 0.000 & 366 & $3.80 \mathrm{E}-03$ & $5.00 \mathrm{E}-07$ & $3.80 \mathrm{E}-03$ & 0.12 & $1.20 \mathrm{E}-03$ & $5.00 \mathrm{E}-07$ & $1.20 \mathrm{E}-03$ & 0.037 \\
\hline $\mathrm{AX}-101$ & $4.39 \mathrm{E}+06$ & No Coatings & 38.1 & 0.0000 & 0.000 & 366 & $3.80 \mathrm{E}-03$ & \begin{tabular}{|l|}
$5.00 \mathrm{E}-07$ \\
\end{tabular} & $3.80 \mathrm{E}-03$ & 0.12 & $1.20 \mathrm{E}-03$ & $5.00 \mathrm{E}-07$ & $1.20 \mathrm{E}-03$ & 0.037 \\
\hline $\mathrm{AX}-102$ & $4.39 \mathrm{E}+06$ & No Coatings & 38.1 & 0.0000 & 0.000 & 366 & $3.80 \mathrm{E}-03$ & \begin{tabular}{|l|}
$5.00 \mathrm{E}-07$ \\
\end{tabular} & $3.80 \mathrm{E}-03$ & 0.12 & $1.20 \mathrm{E}-03$ & $5.00 \mathrm{E}-07$ & $1.20 \mathrm{E}-03$ & 0.037 \\
\hline $\mathrm{AX}-103$ & $4.39 \mathrm{E}+06$ & No Coatings & 38.1 & 0.0000 & 0.000 & 366 & $3.80 \mathrm{E}-03$ & $5.00 \mathrm{E}-07$ & $3.80 \mathrm{E}-03$ & 0.12 & $1.20 \mathrm{E}-03$ & $5.00 \mathrm{E}-07$ & $1.20 \mathrm{E}-03$ & 0.037 \\
\hline AX-104 & $4.39 \mathrm{E}+06$ & No Coatings & 38.1 & 0.0000 & 0.000 & 366 & $3.80 \mathrm{E}-03$ & \begin{tabular}{|l|}
$5.00 \mathrm{E}-07$ \\
\end{tabular} & $3.80 \mathrm{E}-03$ & 0.12 & $1.20 \mathrm{E}-03$ & $5.00 \mathrm{E}-07$ & $1.20 \mathrm{E}-03$ & 0.037 \\
\hline B-101 & $4.43 E+06$ & $3-\mathrm{Ply}+3 / 4$ Mortar & 38.1 & 0.9525 & 1.905 & 404 & $3.80 \mathrm{E}-03$ & $5.00 \mathrm{E}-07$ & $3.80 \mathrm{E}-03$ & 0.12 & $1.20 \mathrm{E}-03$ & $5.00 \mathrm{E}-07$ & $1.20 \mathrm{E}-03$ & 0.037 \\
\hline B-102 & $4.43 \mathrm{E}+06$ & $3-P l y+3 / 4$ Mortar & 38.1 & 0.9525 & 1.905 & 404 & $3.80 \mathrm{E}-03$ & $5.00 \mathrm{E}-07$ & $3.80 \mathrm{E}-03$ & 0.12 & $1.20 \mathrm{E}-03$ & $5.00 \mathrm{E}-07$ & $1.20 \mathrm{E}-03$ & 0.037 \\
\hline B-103 & $4.43 \mathrm{E}+06$ & 3-Ply + 3/4 Mortar & 38.1 & 0.9525 & 1.905 & 404 & $3.80 \mathrm{E}-03$ & $5.00 \mathrm{E}-07$ & $3.80 \mathrm{E}-03$ & 0.12 & $1.20 \mathrm{E}-03$ & $5.00 \mathrm{E}-07$ & $1.20 \mathrm{E}-03$ & 0.037 \\
\hline B-104 & $4.43 \mathrm{E}+06$ & 3-Ply + 3/4 Mortar & 38.1 & 0.9525 & 1.905 & 404 & $3.80 \mathrm{E}-03$ & $5.00 \mathrm{E}-07$ & $3.80 \mathrm{E}-03$ & 0.12 & $1.20 \mathrm{E}-03$ & $5.00 \mathrm{E}-07$ & $1.20 \mathrm{E}-03$ & 0.037 \\
\hline B-105 & $4.43 \mathrm{E}+06$ & 3-Ply + 3/4 Mortar & 38.1 & 0.9525 & 1.905 & 404 & $3.80 \mathrm{E}-03$ & $5.00 \mathrm{E}-07$ & $3.80 \mathrm{E}-03$ & 0.12 & $1.20 \mathrm{E}-03$ & $5.00 \mathrm{E}-07$ & $1.20 \mathrm{E}-03$ & 0.037 \\
\hline B-106 & $4.43 \mathrm{E}+06$ & 3-Ply + 3/4 Mortar & 38.1 & 0.9525 & 1.905 & 404 & $3.80 \mathrm{E}-03$ & $5.00 \mathrm{E}-07$ & $3.80 \mathrm{E}-03$ & 0.12 & $1.20 \mathrm{E}-03$ & $5.00 \mathrm{E}-07$ & $1.20 \mathrm{E}-03$ & 0.037 \\
\hline B-107 & $4.43 \mathrm{E}+06$ & 3-Ply + 3/4 Mortar & 38.1 & 0.9525 & 1.905 & 404 & $3.80 \mathrm{E}-03$ & $5.00 \mathrm{E}-07$ & $3.80 \mathrm{E}-03$ & 0.12 & $1.20 \mathrm{E}-03$ & $5.00 \mathrm{E}-07$ & $1.20 \mathrm{E}-03$ & 0.037 \\
\hline B-108 & $4.43 \mathrm{E}+06$ & 3-Ply + 3/4 Mortar & 38.1 & 0.9525 & 1.905 & 404 & $3.80 \mathrm{E}-03$ & $5.00 \mathrm{E}-07$ & $3.80 \mathrm{E}-03$ & 0.12 & $1.20 \mathrm{E}-03$ & $5.00 \mathrm{E}-07$ & $1.20 \mathrm{E}-03$ & 0.037 \\
\hline B-109 & $4.43 \mathrm{E}+06$ & 3-Ply + 3/4 Mortar & 38.1 & 0.9525 & 1.905 & 404 & $3.80 \mathrm{E}-03$ & $5.00 \mathrm{E}-07$ & $3.80 \mathrm{E}-03$ & 0.12 & $1.20 \mathrm{E}-03$ & $5.00 \mathrm{E}-07$ & $1.20 \mathrm{E}-03$ & 0.037 \\
\hline B-110 & $4.43 \mathrm{E}+06$ & 3-Ply + 3/4 Mortar & 38.1 & 0.9525 & 1.905 & 404 & $3.80 \mathrm{E}-03$ & $5.00 \mathrm{E}-07$ & $3.80 \mathrm{E}-03$ & 0.12 & $1.20 \mathrm{E}-03$ & $5.00 \mathrm{E}-07$ & $1.20 \mathrm{E}-03$ & 0.037 \\
\hline B-111 & $4.43 \mathrm{E}+06$ & 3-Ply + 3/4 Mortar & 38.1 & 0.9525 & 1.905 & 404 & $3.80 \mathrm{E}-03$ & $5.00 \mathrm{E}-07$ & $3.80 \mathrm{E}-03$ & 0.12 & $1.20 \mathrm{E}-03$ & $5.00 \mathrm{E}-07$ & $1.20 \mathrm{E}-03$ & 0.037 \\
\hline B-112 & $4.43 \mathrm{E}+06$ & 3-Ply + 3/4 Mortar & 38.1 & 0.9525 & 1.905 & 404 & $3.80 \mathrm{E}-03$ & $5.00 \mathrm{E}-07$ & $3.80 \mathrm{E}-03$ & 0.12 & $1.20 \mathrm{E}-03$ & $5.00 \mathrm{E}-07$ & $1.20 \mathrm{E}-03$ & 0.037 \\
\hline B-201 & $1.46 \mathrm{E}+05$ & Hardener + 3-Ply & 30.48 & 0.9525 & 0.000 & 335 & $3.80 \mathrm{E}-03$ & $5.00 \mathrm{E}-07$ & $3.80 \mathrm{E}-03$ & 0.12 & $1.20 \mathrm{E}-03$ & $5.00 \mathrm{E}-07$ & $1.20 \mathrm{E}-03$ & 0.037 \\
\hline B-202 & $1.46 \mathrm{E}+05$ & Hardener + 3-Ply & 30.48 & 0.9525 & 0.000 & 335 & $3.80 \mathrm{E}-03$ & $5.00 \mathrm{E}-07$ & $3.80 \mathrm{E}-03$ & 0.12 & $1.20 \mathrm{E}-03$ & $5.00 \mathrm{E}-07$ & $1.20 \mathrm{E}-03$ & 0.037 \\
\hline B-203 & $1.46 \mathrm{E}+05$ & Hardener + 3-Ply & 30.48 & 0.9525 & 0.000 & 335 & $3.80 \mathrm{E}-03$ & $5.00 \mathrm{E}-07$ & $3.80 \mathrm{E}-03$ & 0.12 & $1.20 \mathrm{E}-03$ & $5.00 \mathrm{E}-07$ & $1.20 \mathrm{E}-03$ & 0.037 \\
\hline B-204 & $1.46 \mathrm{E}+05$ & Hardener + 3-Ply & 30.48 & 0.9525 & 0.000 & 335 & $3.80 \mathrm{E}-03$ & $5.00 \mathrm{E}-07$ & $3.80 \mathrm{E}-03$ & 0.12 & $1.20 \mathrm{E}-03$ & $5.00 \mathrm{E}-07$ & $1.20 \mathrm{E}-03$ & 0.037 \\
\hline BX-101 & $4.43 \mathrm{E}+06$ & 3-Ply + 3/4 Mortar & 38.1 & 0.9525 & 1.905 & 404 & $3.80 \mathrm{E}-03$ & $5.00 \mathrm{E}-07$ & $3.80 \mathrm{E}-03$ & 0.12 & $1.20 \mathrm{E}-03$ & $5.00 \mathrm{E}-07$ & $1.20 \mathrm{E}-03$ & 0.037 \\
\hline BX-102 & $4.43 \mathrm{E}+06$ & 3-Ply + 3/4 Mortar & 38.1 & 0.9525 & 1.905 & 404 & $3.80 \mathrm{E}-03$ & $5.00 \mathrm{E}-07$ & $3.80 \mathrm{E}-03$ & 0.12 & $1.20 \mathrm{E}-03$ & $5.00 \mathrm{E}-07$ & $1.20 \mathrm{E}-03$ & 0.037 \\
\hline BX-103 & $4.43 \mathrm{E}+06$ & 3-Ply + 3/4 Mortar & 38.1 & 0.9525 & 1.905 & 404 & $3.80 \mathrm{E}-03$ & $5.00 \mathrm{E}-07$ & $3.80 \mathrm{E}-03$ & 0.12 & $1.20 \mathrm{E}-03$ & $5.00 \mathrm{E}-07$ & $1.20 \mathrm{E}-03$ & 0.037 \\
\hline BX-104 & $4.43 \mathrm{E}+06$ & 3-Ply + 3/4 Mortar & 38.1 & 0.9525 & 1.905 & 404 & $3.80 \mathrm{E}-03$ & $5.00 \mathrm{E}-07$ & $3.80 \mathrm{E}-03$ & 0.12 & $1.20 \mathrm{E}-03$ & $5.00 \mathrm{E}-07$ & $1.20 \mathrm{E}-03$ & 0.037 \\
\hline BX-105 & $4.43 \mathrm{E}+06$ & 3-Ply + 3/4 Mortar & 38.1 & 0.9525 & 1.905 & 404 & $3.80 \mathrm{E}-03$ & $5.00 \mathrm{E}-07$ & $3.80 \mathrm{E}-03$ & 0.12 & $1.20 \mathrm{E}-03$ & $5.00 \mathrm{E}-07$ & $1.20 \mathrm{E}-03$ & 0.037 \\
\hline BX-106 & $4.43 E+06$ & 3-Ply + 3/4 Mortar & 38.1 & 0.9525 & 1.905 & 404 & $3.80 \mathrm{E}-03$ & $5.00 \mathrm{E}-07$ & $3.80 \mathrm{E}-03$ & 0.12 & $1.20 \mathrm{E}-03$ & $5.00 \mathrm{E}-07$ & $1.20 \mathrm{E}-03$ & 0.037 \\
\hline BX-107 & $4.43 \mathrm{E}+06$ & 3-Ply + 3/4 Mortar & 38.1 & 0.9525 & 1.905 & 404 & $3.80 \mathrm{E}-03$ & $5.00 \mathrm{E}-07$ & $3.80 \mathrm{E}-03$ & 0.12 & $1.20 \mathrm{E}-03$ & $5.00 \mathrm{E}-07$ & $1.20 \mathrm{E}-03$ & 0.037 \\
\hline BX-108 & $4.43 \mathrm{E}+06$ & 3-Ply + 3/4 Mortar & 38.1 & 0.9525 & 1.905 & 404 & $3.80 \mathrm{E}-03$ & $5.00 \mathrm{E}-07$ & $3.80 \mathrm{E}-03$ & 0.12 & $1.20 \mathrm{E}-03$ & $5.00 \mathrm{E}-07$ & $1.20 \mathrm{E}-03$ & 0.037 \\
\hline BX-109 & $4.43 E+06$ & 3-Ply + 3/4 Mortar & 38.1 & 0.9525 & 1.905 & 404 & $3.80 \mathrm{E}-03$ & \begin{tabular}{|l|}
$5.00 \mathrm{E}-07$ \\
\end{tabular} & $3.80 \mathrm{E}-03$ & 0.12 & $1.20 \mathrm{E}-03$ & $5.00 \mathrm{E}-07$ & $1.20 \mathrm{E}-03$ & 0.037 \\
\hline $\mathrm{BX}-110$ & $4.43 \mathrm{E}+06$ & 3-Ply + 3/4 Mortar & 38.1 & 0.9525 & 1.905 & 404 & $3.80 \mathrm{E}-03$ & $5.00 \mathrm{E}-07$ & $3.80 \mathrm{E}-03$ & 0.12 & $1.20 \mathrm{E}-03$ & $5.00 \mathrm{E}-07$ & $1.20 \mathrm{E}-03$ & 0.037 \\
\hline BX-111 & $4.43 \mathrm{E}+06$ & 3-Ply + 3/4 Mortar & 38.1 & 0.9525 & 1.905 & 404 & $3.80 \mathrm{E}-03$ & \begin{tabular}{|l|}
$5.00 \mathrm{E}-07$ \\
\end{tabular} & $3.80 \mathrm{E}-03$ & 0.12 & $1.20 \mathrm{E}-03$ & $5.00 \mathrm{E}-07$ & $1.20 \mathrm{E}-03$ & 0.037 \\
\hline BX-112 & $4.43 \mathrm{E}+06$ & 3-Ply + 3/4 Mortar & 38.1 & 0.9525 & 1.905 & 404 & $3.80 \mathrm{E}-03$ & $5.00 \mathrm{E}-07$ & $3.80 \mathrm{E}-03$ & 0.12 & $1.20 \mathrm{E}-03$ & $5.00 \mathrm{E}-07$ & $1.20 \mathrm{E}-03$ & 0.037 \\
\hline BY-101 & $4.38 \mathrm{E}+06$ & Hardener + 3-Ply $+3 / 4$ Gunite & 38.1 & 0.9525 & 1.905 & 427 & $3.80 \mathrm{E}-03$ & $5.00 \mathrm{E}-07$ & $3.80 \mathrm{E}-03$ & 0.12 & $1.20 \mathrm{E}-03$ & $5.00 \mathrm{E}-07$ & $1.20 \mathrm{E}-03$ & 0.037 \\
\hline BY-102 & $4.38 \mathrm{E}+06$ & Hardener + 3-Ply + 3/4 Gunite & 38.1 & 0.9525 & 1.905 & 427 & $3.80 \mathrm{E}-03$ & \begin{tabular}{|l|}
$5.00 \mathrm{E}-07$ \\
\end{tabular} & $3.80 \mathrm{E}-03$ & 0.12 & $1.20 \mathrm{E}-03$ & $5.00 \mathrm{E}-07$ & $1.20 \mathrm{E}-03$ & 0.037 \\
\hline BY-103 & $4.38 \mathrm{E}+06$ & Hardener +3 -Ply $+3 / 4$ Gunite & 38.1 & 0.9525 & 1.905 & 427 & $3.80 \mathrm{E}-03$ & $5.00 \mathrm{E}-07$ & $3.80 \mathrm{E}-03$ & 0.12 & $1.20 \mathrm{E}-03$ & $5.00 \mathrm{E}-07$ & $1.20 \mathrm{E}-03$ & 0.037 \\
\hline
\end{tabular}


Table B-1. The Thickness of Dome Coatings for 149 Single-Shell Tanks and Diffusion Coefficients for Hydrogen and Methane.

\begin{tabular}{|c|c|c|c|c|c|c|c|c|c|c|c|c|c|c|}
\hline Tank & $\begin{array}{c}\text { Dome } \\
\text { Area } \\
\left(\mathrm{cm}^{2}\right) \\
\end{array}$ & Tank Dome Coatings & $\begin{array}{c}\text { Concrete } \\
\text { Thick } \\
\text { (cm) }\end{array}$ & $\begin{array}{c}\text { Asphaltic } \\
\text { Thick } \\
\text { (cm) }\end{array}$ & $\begin{array}{l}\text { Gunite } \\
\text { Thick } \\
\text { (cm) } \\
\end{array}$ & $\begin{array}{c}\text { Soil } \\
\text { Thick } \\
\text { (cm) }\end{array}$ & $\begin{array}{l}\text { Concrete } \\
\text { De of } \mathrm{H}_{2} \\
\left(\mathrm{~cm}^{2} / \mathrm{sec}\right)\end{array}$ & $\begin{array}{c}\text { Asphaltic } \\
\text { De of } \mathrm{H}_{2} \\
\left(\mathrm{~cm}^{2} / \mathrm{sec}\right)\end{array}$ & $\begin{array}{c}\text { Gunite } \\
\text { De of } \mathrm{H}_{2} \\
\left(\mathrm{~cm}^{2} / \mathrm{sec}\right)\end{array}$ & $\begin{array}{c}\text { Soil } \\
\text { De of } \mathrm{H}_{2} \\
\left(\mathrm{~cm}^{2} / \mathrm{sec}\right)\end{array}$ & $\begin{array}{c}\text { Concrete De } \\
\text { of } \mathrm{CH}_{4} \\
\left(\mathrm{~cm}^{2} / \mathrm{sec}\right) \\
\end{array}$ & $\begin{array}{c}\text { Asphaltic } \\
\text { De of CH } \\
\left(\mathrm{cm}^{2} / \mathrm{sec}\right) \\
\end{array}$ & $\begin{array}{c}\text { Gunite De } \\
\text { of } \mathrm{CH}_{4} \\
\left(\mathrm{~cm}^{2} / \mathrm{sec}\right)\end{array}$ & $\begin{array}{c}\begin{array}{c}\text { Soil } \\
\mathrm{De} \mathrm{of}^{\mathrm{CH}} \mathrm{H}_{4} \\
\left(\mathrm{~cm}^{2} / \mathrm{sec}\right)\end{array} \\
\end{array}$ \\
\hline BY-104 & $4.38 \mathrm{E}+06$ & Hardener + 3-Ply + 3/4 Gunite & 38.1 & 0.9525 & 1.905 & 427 & $3.80 \mathrm{E}-03$ & $5.00 \mathrm{E}-07$ & $3.80 \mathrm{E}-03$ & 0.12 & $1.20 \mathrm{E}-03$ & $5.00 \mathrm{E}-07$ & $1.20 \mathrm{E}-03$ & 0.037 \\
\hline BY-105 & $4.38 \mathrm{E}+06$ & Hardener + 3-Ply + 3/4 Gunite & 38.1 & 0.9525 & 1.905 & 427 & $3.80 \mathrm{E}-03$ & $5.00 \mathrm{E}-07$ & $3.80 \mathrm{E}-03$ & 0.12 & $1.20 \mathrm{E}-03$ & $5.00 \mathrm{E}-07$ & $1.20 \mathrm{E}-03$ & 0.037 \\
\hline BY-106 & $4.38 \mathrm{E}+06$ & Hardener + 3-Ply + 3/4 Gunite & 38.1 & 0.9525 & 1.905 & 427 & $3.80 \mathrm{E}-03$ & $5.00 \mathrm{E}-07$ & $3.80 \mathrm{E}-03$ & 0.12 & $1.20 \mathrm{E}-03$ & $5.00 \mathrm{E}-07$ & $1.20 \mathrm{E}-03$ & 0.037 \\
\hline BY-107 & $4.38 \mathrm{E}+06$ & Hardener + 3-Ply + 3/4 Gunite & 38.1 & 0.9525 & 1.905 & 427 & $3.80 \mathrm{E}-03$ & $5.00 \mathrm{E}-07$ & $3.80 \mathrm{E}-03$ & 0.12 & $1.20 \mathrm{E}-03$ & $5.00 \mathrm{E}-07$ & $1.20 \mathrm{E}-03$ & 0.037 \\
\hline BY-108 & $4.38 \mathrm{E}+06$ & Hardener + 3-Ply + 3/4 Gunite & 38.1 & 0.9525 & 1.905 & 427 & $3.80 \mathrm{E}-03$ & $5.00 \mathrm{E}-07$ & $3.80 \mathrm{E}-03$ & 0.12 & $1.20 \mathrm{E}-03$ & $5.00 \mathrm{E}-07$ & $1.20 \mathrm{E}-03$ & 0.037 \\
\hline BY-109 & $4.38 \mathrm{E}+06$ & Hardener + 3-Ply + 3/4 Gunite & 38.1 & 0.9525 & 1.905 & 427 & $3.80 \mathrm{E}-03$ & $5.00 \mathrm{E}-07$ & $3.80 \mathrm{E}-03$ & 0.12 & $1.20 \mathrm{E}-03$ & $5.00 \mathrm{E}-07$ & $1.20 \mathrm{E}-03$ & 0.037 \\
\hline BY-110 & $4.38 \mathrm{E}+06$ & Hardener + 3-Ply + 3/4 Gunite & 38.1 & 0.9525 & 1.905 & 427 & $3.80 \mathrm{E}-03$ & $5.00 \mathrm{E}-07$ & $3.80 \mathrm{E}-03$ & 0.12 & $1.20 \mathrm{E}-03$ & $5.00 \mathrm{E}-07$ & $1.20 \mathrm{E}-03$ & 0.037 \\
\hline BY-111 & $4.38 \mathrm{E}+06$ & Hardener + 3-Ply $+3 / 4$ Gunite & 38.1 & 0.9525 & 1.905 & 427 & $3.80 \mathrm{E}-03$ & $5.00 \mathrm{E}-07$ & $3.80 \mathrm{E}-03$ & 0.12 & $1.20 \mathrm{E}-03$ & $5.00 \mathrm{E}-07$ & $1.20 \mathrm{E}-03$ & 0.037 \\
\hline BY-112 & $4.38 \mathrm{E}+06$ & Hardener + 3-Ply $+3 / 4$ Gunite & 38.1 & 0.9525 & 1.905 & 427 & $3.80 \mathrm{E}-03$ & $5.00 \mathrm{E}-07$ & $3.80 \mathrm{E}-03$ & 0.12 & $1.20 \mathrm{E}-03$ & $5.00 \mathrm{E}-07$ & $1.20 \mathrm{E}-03$ & 0.037 \\
\hline $\mathrm{C}-101$ & $4.43 \mathrm{E}+06$ & 3-Ply $+3 / 4$ Mortar & 38.1 & 0.9525 & 1.905 & 404 & $3.80 \mathrm{E}-03$ & $5.00 \mathrm{E}-07$ & $3.80 \mathrm{E}-03$ & 0.12 & $1.20 \mathrm{E}-03$ & $5.00 \mathrm{E}-07$ & $1.20 \mathrm{E}-03$ & 0.037 \\
\hline $\mathrm{C}-102$ & $4.43 \mathrm{E}+06$ & 3-Ply + 3/4 Mortar & 38.1 & 0.9525 & 1.905 & 404 & $3.80 \mathrm{E}-03$ & $5.00 \mathrm{E}-07$ & $3.80 \mathrm{E}-03$ & 0.12 & $1.20 \mathrm{E}-03$ & $5.00 \mathrm{E}-07$ & $1.20 \mathrm{E}-03$ & 0.037 \\
\hline$C-103$ & $4.43 \mathrm{E}+06$ & 3-Ply + 3/4 Mortar & 38.1 & 0.9525 & 1.905 & 404 & $3.80 \mathrm{E}-03$ & $5.00 \mathrm{E}-07$ & $3.80 \mathrm{E}-03$ & 0.12 & $1.20 \mathrm{E}-03$ & $5.00 \mathrm{E}-07$ & $1.20 \mathrm{E}-03$ & 0.037 \\
\hline $\mathrm{C}-104$ & $4.43 \mathrm{E}+06$ & 3-Ply $+3 / 4$ Mortar & 38.1 & 0.9525 & 1.905 & 404 & $3.80 \mathrm{E}-03$ & $5.00 \mathrm{E}-07$ & $3.80 \mathrm{E}-03$ & 0.12 & $1.20 \mathrm{E}-03$ & 5.00E-07 & $1.20 \mathrm{E}-03$ & 0.037 \\
\hline$C-105$ & $4.43 \mathrm{E}+06$ & 3-Ply + 3/4 Mortar & 38.1 & 0.9525 & 1.905 & 404 & $3.80 \mathrm{E}-03$ & $5.00 \mathrm{E}-07$ & $3.80 \mathrm{E}-03$ & 0.12 & $1.20 \mathrm{E}-03$ & $5.00 \mathrm{E}-07$ & $1.20 \mathrm{E}-03$ & 0.037 \\
\hline C-106 & $4.43 \mathrm{E}+06$ & 3-Ply + 3/4 Mortar & 38.1 & 0.9525 & 1.905 & 404 & $3.80 \mathrm{E}-03$ & $5.00 \mathrm{E}-07$ & $3.80 \mathrm{E}-03$ & 0.12 & $1.20 \mathrm{E}-03$ & $5.00 \mathrm{E}-07$ & $1.20 \mathrm{E}-03$ & 0.037 \\
\hline C-107 & $4.43 \mathrm{E}+06$ & 3-Ply + 3/4 Mortar & 38.1 & 0.9525 & 1.905 & 404 & $3.80 \mathrm{E}-03$ & $5.00 \mathrm{E}-07$ & $3.80 \mathrm{E}-03$ & 0.12 & $1.20 \mathrm{E}-03$ & $5.00 \mathrm{E}-07$ & $1.20 \mathrm{E}-03$ & 0.037 \\
\hline C-108 & $4.43 \mathrm{E}+06$ & 3-Ply + 3/4 Mortar & 38.1 & 0.9525 & 1.905 & 404 & $3.80 \mathrm{E}-03$ & $5.00 \mathrm{E}-07$ & $3.80 \mathrm{E}-03$ & 0.12 & $1.20 \mathrm{E}-03$ & $5.00 \mathrm{E}-07$ & $1.20 \mathrm{E}-03$ & 0.037 \\
\hline C-109 & $4.43 \mathrm{E}+06$ & 3-Ply + 3/4 Mortar & 38.1 & 0.9525 & 1.905 & 404 & $3.80 \mathrm{E}-03$ & $5.00 \mathrm{E}-07$ & $3.80 \mathrm{E}-03$ & 0.12 & $1.20 \mathrm{E}-03$ & $5.00 \mathrm{E}-07$ & $1.20 \mathrm{E}-03$ & 0.037 \\
\hline$C-110$ & $4.43 \mathrm{E}+06$ & 3-Ply + 3/4 Mortar & 38.1 & 0.9525 & 1.905 & 404 & $3.80 \mathrm{E}-03$ & $5.00 \mathrm{E}-07$ & $3.80 \mathrm{E}-03$ & 0.12 & $1.20 \mathrm{E}-03$ & $5.00 \mathrm{E}-07$ & $1.20 \mathrm{E}-03$ & 0.037 \\
\hline C-111 & $4.43 \mathrm{E}+06$ & 3-Ply + 3/4 Mortar & 38.1 & 0.9525 & 1.905 & 404 & $3.80 \mathrm{E}-03$ & $5.00 \mathrm{E}-07$ & $3.80 \mathrm{E}-03$ & 0.12 & $1.20 \mathrm{E}-03$ & $5.00 \mathrm{E}-07$ & $1.20 \mathrm{E}-03$ & 0.037 \\
\hline $\mathrm{C}-112$ & $4.43 \mathrm{E}+06$ & 3-Ply + 3/4 Mortar & 38.1 & 0.9525 & 1.905 & 404 & $3.80 \mathrm{E}-03$ & $5.00 \mathrm{E}-07$ & $3.80 \mathrm{E}-03$ & 0.12 & $1.20 \mathrm{E}-03$ & $5.00 \mathrm{E}-07$ & $1.20 \mathrm{E}-03$ & 0.037 \\
\hline $\mathrm{C}-201$ & $1.46 \mathrm{E}+05$ & Hardener + 3-Ply & 30.48 & 0.9525 & 0.000 & 335 & $3.80 \mathrm{E}-03$ & $5.00 \mathrm{E}-07$ & $3.80 \mathrm{E}-03$ & 0.12 & $1.20 \mathrm{E}-03$ & $5.00 \mathrm{E}-07$ & \begin{tabular}{|l|}
$1.20 \mathrm{E}-03$ \\
\end{tabular} & 0.037 \\
\hline $\mathrm{C}-202$ & $1.46 \mathrm{E}+05$ & Hardener + 3-Ply & 30.48 & 0.9525 & 0.000 & 335 & $3.80 \mathrm{E}-03$ & $5.00 \mathrm{E}-07$ & $3.80 \mathrm{E}-03$ & 0.12 & $1.20 \mathrm{E}-03$ & $5.00 \mathrm{E}-07$ & $1.20 \mathrm{E}-03$ & 0.037 \\
\hline $\mathrm{C}-203$ & $1.46 \mathrm{E}+05$ & Hardener + 3-Ply & 30.48 & 0.9525 & 0.000 & 335 & $3.80 \mathrm{E}-03$ & $5.00 \mathrm{E}-07$ & $3.80 \mathrm{E}-03$ & 0.12 & $1.20 \mathrm{E}-03$ & $5.00 \mathrm{E}-07$ & $1.20 \mathrm{E}-03$ & 0.037 \\
\hline C-204 & $1.46 \mathrm{E}+05$ & Hardener + 3-Ply & 30.48 & 0.9525 & 0.000 & 335 & $3.80 \mathrm{E}-03$ & $5.00 \mathrm{E}-07$ & $3.80 \mathrm{E}-03$ & 0.12 & $1.20 \mathrm{E}-03$ & $5.00 \mathrm{E}-07$ & $1.20 \mathrm{E}-03$ & 0.037 \\
\hline S-101 & $4.38 \mathrm{E}+06$ & Hardener + 3-Ply + 3/4 Gunite & 38.1 & 0.9525 & 1.905 & 427 & $3.80 \mathrm{E}-03$ & $5.00 \mathrm{E}-07$ & $3.80 \mathrm{E}-03$ & 0.12 & $1.20 \mathrm{E}-03$ & $5.00 \mathrm{E}-07$ & $1.20 \mathrm{E}-03$ & 0.037 \\
\hline S-102 & $4.38 \mathrm{E}+06$ & Hardener + 3-Ply $+3 / 4$ Gunite & 38.1 & 0.9525 & 1.905 & 427 & $3.80 \mathrm{E}-03$ & $5.00 \mathrm{E}-07$ & $3.80 \mathrm{E}-03$ & 0.12 & $1.20 \mathrm{E}-03$ & $5.00 \mathrm{E}-07$ & $1.20 \mathrm{E}-03$ & 0.037 \\
\hline S-103 & $4.38 \mathrm{E}+06$ & Hardener + 3-Ply $+3 / 4$ Gunite & 38.1 & 0.9525 & 1.905 & 427 & $3.80 \mathrm{E}-03$ & $5.00 \mathrm{E}-07$ & $3.80 \mathrm{E}-03$ & 0.12 & $1.20 \mathrm{E}-03$ & $5.00 \mathrm{E}-07$ & $1.20 \mathrm{E}-03$ & 0.037 \\
\hline S-104 & $4.38 \mathrm{E}+06$ & Hardener + 3-Ply $+3 / 4$ Gunite & 38.1 & 0.9525 & 1.905 & 427 & $3.80 \mathrm{E}-03$ & $5.00 \mathrm{E}-07$ & $3.80 \mathrm{E}-03$ & 0.12 & $1.20 \mathrm{E}-03$ & $5.00 \mathrm{E}-07$ & $1.20 \mathrm{E}-03$ & 0.037 \\
\hline S-105 & $4.38 \mathrm{E}+06$ & Hardener + 3-Ply + 3/4 Gunite & 38.1 & 0.9525 & 1.905 & 427 & $3.80 \mathrm{E}-03$ & $5.00 \mathrm{E}-07$ & $3.80 \mathrm{E}-03$ & 0.12 & $1.20 \mathrm{E}-03$ & $5.00 \mathrm{E}-07$ & $1.20 \mathrm{E}-03$ & 0.037 \\
\hline S-106 & $4.38 \mathrm{E}+06$ & Hardener + 3-Ply + 3/4 Gunite & 38.1 & 0.9525 & 1.905 & 427 & $3.80 \mathrm{E}-03$ & $5.00 \mathrm{E}-07$ & $3.80 \mathrm{E}-03$ & 0.12 & $1.20 \mathrm{E}-03$ & $5.00 \mathrm{E}-07$ & $1.20 \mathrm{E}-03$ & 0.037 \\
\hline S-107 & $4.38 \mathrm{E}+06$ & Hardener + 3-Ply $+3 / 4$ Gunite & 38.1 & 0.9525 & 1.905 & 427 & $3.80 \mathrm{E}-03$ & $5.00 \mathrm{E}-07$ & $3.80 \mathrm{E}-03$ & 0.12 & $1,20 \mathrm{E}-03$ & $5.00 \mathrm{E}-07$ & $1.20 \mathrm{E}-03$ & 0.037 \\
\hline S-108 & $4.38 \mathrm{E}+06$ & Hardener + 3-Ply + 3/4 Gunite & 38.1 & 0.9525 & 1.905 & 427 & $3.80 \mathrm{E}-03$ & $5.00 \mathrm{E}-07$ & $3.80 \mathrm{E}-03$ & 0.12 & $1.20 \mathrm{E}-03$ & $5.00 \mathrm{E}-07$ & $1.20 \mathrm{E}-03$ & 0.037 \\
\hline S-109 & $4.38 \mathrm{E}+06$ & Hardener $+3-\mathrm{Ply}+3 / 4$ Gunite & 38.1 & 0.9525 & 1.905 & 427 & $3.80 \mathrm{E}-03$ & $5.00 \mathrm{E}-07$ & $3.80 \mathrm{E}-03$ & 0.12 & $1.20 \mathrm{E}-03$ & $5.00 \mathrm{E}-07$ & $1.20 \mathrm{E}-03$ & 0.037 \\
\hline$S-110$ & $4.38 \mathrm{E}+06$ & Hardener + 3-Ply + 3/4 Gunite & 38.1 & 0.9525 & 1.905 & 427 & $3.80 \mathrm{E}-03$ & $5.00 \mathrm{E}-07$ & $3.80 \mathrm{E}-03$ & 0.12 & $1.20 \mathrm{E}-03$ & $5.00 \mathrm{E}-07$ & $1,20 \mathrm{E}-03$ & 0.037 \\
\hline $\mathrm{S}-111$ & $4.38 \mathrm{E}+06$ & Hardener + 3-Ply + 3/4 Gunite & 38.1 & 0.9525 & 1.905 & 427 & $3.80 \mathrm{E}-03$ & $5.00 \mathrm{E}-07$ & $3.80 \mathrm{E}-03$ & 0.12 & $1.20 \mathrm{E}-03$ & $5.00 \mathrm{E}-07$ & $1.20 \mathrm{E}-03$ & 0.037 \\
\hline $\mathrm{S}-112$ & $4.38 \mathrm{E}+06$ & Hardener + 3-Ply + 3/4 Gunite & 38.1 & 0.9525 & 1.905 & 427 & $3.80 \mathrm{E}-03$ & $5.00 \mathrm{E}-07$ & $3.80 \mathrm{E}-03$ & 0.12 & $1.20 \mathrm{E}-03$ & 5.00E-07 & $1.20 \mathrm{E}-03$ & 0.037 \\
\hline SX-101 & $4.39 \mathrm{E}+06$ & Hardener & 38.1 & 0.0000 & 0.000 & 366 & $3.80 \mathrm{E}-03$ & $5.00 \mathrm{E}-07$ & 3.80E-03 & 0.12 & $1.20 \mathrm{E}-03$ & $5.00 \mathrm{E}-07$ & $1.20 \mathrm{E}-03$ & 0.037 \\
\hline SX-102 & $4.39 \mathrm{E}+06$ & Hardener & 38.1 & 0.0000 & 0.000 & 366 & $3.80 \mathrm{E}-03$ & $5.00 \mathrm{E}-07$ & $3.80 \mathrm{E}-03$ & 0.12 & $1.20 \mathrm{E}-03$ & $5.00 \mathrm{E}-07$ & $1.20 \mathrm{E}-03$ & 0.037 \\
\hline SX-103 & $4.39 \mathrm{E}+06$ & Hardener & 38.1 & 0.0000 & 0.000 & 366 & $3.80 \mathrm{E}-03$ & $5.00 \mathrm{E}-07$ & $3.80 \mathrm{E}-03$ & 0.12 & $1.20 \mathrm{E}-03$ & $5.00 \mathrm{E}-07$ & $1.20 \mathrm{E}-03$ & 0.037 \\
\hline SX-104 & $4.39 \mathrm{E}+06$ & Hardener & 38.1 & 0.0000 & 0.000 & 366 & $3.80 \mathrm{E}-03$ & $5.00 \mathrm{E}-07$ & $3.80 \mathrm{E}-03$ & 0.12 & $1.20 \mathrm{E}-03$ & $5.00 \mathrm{E}-07$ & $1.20 \mathrm{E}-03$ & 0.037 \\
\hline SX-105 & $4.39 \mathrm{E}+06$ & Hardener & 38.1 & 0.0000 & 0.000 & 366 & $3.80 \mathrm{E}-03$ & $5.00 \mathrm{E}-07$ & $3.80 \mathrm{E}-03$ & 0.12 & $1.20 \mathrm{E}-03$ & $5.00 \mathrm{E}-07$ & $1.20 \mathrm{E}-03$ & 0.037 \\
\hline
\end{tabular}


Table B-1. The Thickness of Dome Coatings for 149 Single-Shell Tanks and Diffusion Coefficients for Hydrogen and Methane.

\begin{tabular}{|c|c|c|c|c|c|c|c|c|c|c|c|c|c|c|}
\hline Tank & $\begin{array}{l}\text { Dome } \\
\text { Area } \\
\left(\mathrm{cm}^{2}\right)\end{array}$ & Tank Dome Coatings & $\begin{array}{l}\text { Concrete } \\
\text { Thick } \\
\text { (cm) }\end{array}$ & $\begin{array}{c}\text { Asphaltic } \\
\text { Thick } \\
\text { (cm) }\end{array}$ & $\begin{array}{l}\text { Gunite } \\
\text { Thick } \\
\text { (cm) }\end{array}$ & $\begin{array}{l}\text { Soil } \\
\text { Thick } \\
\text { (cm) }\end{array}$ & $\begin{array}{l}\text { Concrete } \\
\mathrm{De} \text { of } \mathrm{H}_{2} \\
\left(\mathrm{~cm}^{2} / \mathrm{sec}\right)\end{array}$ & $\begin{array}{l}\text { Asphaltic } \\
\text { De of } \mathrm{H}_{2} \\
\left(\mathrm{~cm}^{2} / \mathrm{sec}\right)\end{array}$ & $\begin{array}{c}\text { Gunite } \\
\text { De of } \mathrm{H}_{2} \\
\left(\mathrm{~cm}^{2} / \mathrm{sec}\right)\end{array}$ & $\begin{array}{c}\text { Soil } \\
\text { De of } \mathrm{H}_{2} \\
\left(\mathrm{~cm}^{2} / \mathrm{sec}\right)\end{array}$ & $\begin{array}{c}\text { Concrete De } \\
\text { of } \mathrm{CH}_{4} \\
\left(\mathrm{~cm}^{2} / \mathrm{sec}\right)\end{array}$ & $\begin{array}{l}\text { Asphaltic } \\
\mathrm{De} \text { of } \mathrm{CH}_{4} \\
\left(\mathrm{~cm}^{2} / \mathrm{sec}\right)\end{array}$ & $\left.\begin{array}{c}\text { Gunite De } \\
\text { of } \mathrm{CH}_{4} \\
\left(\mathrm{~cm}^{2} / \mathrm{sec}\right)\end{array}\right)$ & $\begin{array}{c}\text { Soil } \\
\mathrm{De}^{\text {of } \mathrm{CH}_{4}} \\
\left(\mathrm{~cm}^{2} / \mathrm{sec}\right)\end{array}$ \\
\hline SX-106 & $4.39 \mathrm{E}+06$ & Hardener & 38.1 & 0.0000 & 0.000 & 366 & $3.80 \mathrm{E}-03$ & $5.00 \mathrm{E}-07$ & $3.80 \mathrm{E}-03$ & 0.12 & $1.20 \mathrm{E}-03$ & $5.00 \mathrm{E}-07$ & $1.20 \mathrm{E}-03$ & 0.037 \\
\hline SX-107 & $4.39 \mathrm{E}+06$ & Hardener & 38.1 & 0.0000 & 0.000 & 366 & $3.80 \mathrm{E}-03$ & \begin{tabular}{|l|}
$5.00 \mathrm{E}-07$ \\
\end{tabular} & $3.80 \mathrm{E}-03$ & 0.12 & $1.20 \mathrm{E}-03$ & $5.00 \mathrm{E}-07$ & \begin{tabular}{|l|}
$1.20 \mathrm{E}-03$ \\
\end{tabular} & 0.037 \\
\hline SX-108 & $4.39 \mathrm{E}+06$ & Hardener & 38.1 & 0.0000 & 0.000 & 366 & $3.80 \mathrm{E}-03$ & $5.00 \mathrm{E}-07$ & $3.80 \mathrm{E}-03$ & 0.12 & $1.20 \mathrm{E}-03$ & $5.00 \mathrm{E}-07$ & $1.20 \mathrm{E}-03$ & 0.037 \\
\hline SX-109 & $4.39 E+06$ & Hardener & 38.1 & 0.0000 & 0.000 & 366 & $3.80 \mathrm{E}-03$ & $5.00 \mathrm{E}-07$ & $3.80 \mathrm{E}-03$ & 0.12 & $1.20 \mathrm{E}-03$ & $5.00 \mathrm{E}-07$ & $1.20 \mathrm{E}-03$ & 0.037 \\
\hline SX-110 & $4.39 \mathrm{E}+06$ & Hardener & 38.1 & 0.0000 & 0.000 & 366 & $3.80 \mathrm{E}-03$ & $5.00 \mathrm{E}-07$ & $3.80 \mathrm{E}-03$ & 0.12 & $1.20 \mathrm{E}-03$ & $5.00 \mathrm{E}-07$ & $1.20 \mathrm{E}-03$ & 0.037 \\
\hline SX-111 & $4.39 \mathrm{E}+06$ & Hardener & 38.1 & 0.0000 & 0.000 & 366 & $3.80 \mathrm{E}-03$ & $5.00 \mathrm{E}-07$ & $3.80 \mathrm{E}-03$ & 0.12 & $1.20 \mathrm{E}-03$ & $5.00 \mathrm{E}-07$ & $1.20 \mathrm{E}-03$ & 0.037 \\
\hline $\mathrm{SX}-112$ & $4.39 \mathrm{E}+06$ & Hardener & 38.1 & 0.0000 & 0.000 & 366 & $3.80 \mathrm{E}-03$ & $5.00 \mathrm{E}-07$ & $3.80 \mathrm{E}-03$ & 0.12 & $1.20 \mathrm{E}-03$ & $5.00 \mathrm{E}-07$ & $1.20 \mathrm{E}-03$ & 0.037 \\
\hline SX-113 & $4.39 \mathrm{E}+06$ & Hardener & 38.1 & 0.0000 & 0.000 & 366 & $3.80 \mathrm{E}-03$ & $5.00 \mathrm{E}-07$ & $3.80 \mathrm{E}-03$ & 0.12 & $1.20 \mathrm{E}-03$ & $5.00 \mathrm{E}-07$ & $1.20 \mathrm{E}-03$ & 0.037 \\
\hline SX-114 & $4.39 \mathrm{E}+06$ & Hardener & 38.1 & 0.0000 & 0.000 & 366 & $3.80 \mathrm{E}-03$ & $5.00 \mathrm{E}-07$ & $3.80 \mathrm{E}-03$ & 0.12 & $1.20 \mathrm{E}-03$ & $5.00 \mathrm{E}-07$ & \begin{tabular}{|l|}
$1.20 \mathrm{E}-03$ \\
\end{tabular} & 0.037 \\
\hline SX-115 & $4.39 \mathrm{E}+06$ & Hardener & 38.1 & 0.0000 & 0.000 & 366 & $3.80 \mathrm{E}-03$ & $5.00 \mathrm{E}-07$ & $3.80 \mathrm{E}-03$ & 0.12 & $1.20 \mathrm{E}-03$ & $5.00 \mathrm{E}-07$ & $1.20 \mathrm{E}-03$ & 0.037 \\
\hline $\mathrm{T}-101$ & $4.43 \mathrm{E}+06$ & 3-Ply +3/4 Mortar & 38.1 & 0.9525 & 1.905 & 404 & $3.80 \mathrm{E}-03$ & $5.00 \mathrm{E}-07$ & $3.80 \mathrm{E}-03$ & 0.12 & $1.20 \mathrm{E}-03$ & $5.00 \mathrm{E}-07$ & \begin{tabular}{|l|}
$1.20 \mathrm{E}-03$ \\
\end{tabular} & 0.037 \\
\hline $\mathrm{T}-102$ & $4.43 E+06$ & 3-Ply + 3/4 Mortar & 38.1 & 0.9525 & 1.905 & 404 & $3.80 \mathrm{E}-03$ & $5.00 \mathrm{E}-07$ & $3.80 \mathrm{E}-03$ & 0.12 & $1.20 \mathrm{E}-03$ & $5.00 \mathrm{E}-07$ & $1.20 \mathrm{E}-03$ & 0.037 \\
\hline $\mathrm{T}-103$ & $4.43 E+06$ & $3-\mathrm{Ply}+3 / 4$ Mortar & 38.1 & 0.9525 & 1.905 & 404 & $3.80 \mathrm{E}-03$ & $5.00 \mathrm{E}-07$ & $3.80 \mathrm{E}-03$ & 0.12 & $1.20 \mathrm{E}-03$ & $5.00 \mathrm{E}-07$ & $1.20 \mathrm{E}-03$ & 0.037 \\
\hline $\mathrm{T}-104$ & $4.43 E+06$ & 3-Ply + 3/4 Mortar & 38.1 & 0.9525 & 1.905 & 404 & $3.80 \mathrm{E}-03$ & $5.00 \mathrm{E}-07$ & $3.80 \mathrm{E}-03$ & 0.12 & $1.20 \mathrm{E}-03$ & $5.00 \mathrm{E}-07$ & $1.20 \mathrm{E}-03$ & 0.037 \\
\hline $\mathrm{T}-105$ & $4.43 \mathrm{E}+06$ & 3-Ply + 3/4 Mortar & 38.1 & 0.9525 & 1.905 & 404 & $3.80 \mathrm{E}-03$ & $5.00 \mathrm{E}-07$ & $3.80 \mathrm{E}-03$ & 0.12 & $1.20 \mathrm{E}-03$ & $5.00 \mathrm{E}-07$ & $1.20 \mathrm{E}-03$ & 0.037 \\
\hline $\mathrm{T}-106$ & $4.43 \mathrm{E}+06$ & 3-Ply + 3/4 Mortar & 38.1 & 0.9525 & 1.905 & 404 & $3.80 \mathrm{E}-03$ & $5.00 \mathrm{E}-07$ & $3.80 \mathrm{E}-03$ & 0.12 & $1.20 \mathrm{E}-03$ & $5.00 \mathrm{E}-07$ & $1.20 \mathrm{E}-03$ & 0.037 \\
\hline $\mathrm{T}-107$ & $4.43 \mathrm{E}+06$ & 3-Ply + 3/4 Mortar & 38.1 & 0.9525 & 1.905 & 404 & $3.80 \mathrm{E}-03$ & $5.00 \mathrm{E}-07$ & $3.80 \mathrm{E}-03$ & 0.12 & $1.20 \mathrm{E}-03$ & $5.00 \mathrm{E}-07$ & $1.20 \mathrm{E}-03$ & 0.037 \\
\hline $\mathrm{T}-108$ & $4.43 \mathrm{E}+06$ & 3-Ply + 3/4 Mortar & 38.1 & 0.9525 & 1.905 & 404 & $3.80 \mathrm{E}-03$ & $5.00 \mathrm{E}-07$ & $3.80 \mathrm{E}-03$ & 0.12 & $1.20 \mathrm{E}-03$ & $5.00 \mathrm{E}-07$ & $1.20 \mathrm{E}-03$ & 0.037 \\
\hline T-109 & $4.43 E+06$ & 3-Ply + 3/4 Mortar & 38.1 & 0.9525 & 1.905 & 404 & $3.80 \mathrm{E}-03$ & $5.00 \mathrm{E}-07$ & $3.80 \mathrm{E}-03$ & 0.12 & $1.20 \mathrm{E}-03$ & $5.00 \mathrm{E}-07$ & $1.20 \mathrm{E}-03$ & 0.037 \\
\hline$T-110$ & $4.43 E+06$ & 3-Ply + 3/4 Mortar & 38.1 & 0.9525 & 1.905 & 404 & $3.80 \mathrm{E}-03$ & $5.00 \mathrm{E}-07$ & $3.80 \mathrm{E}-03$ & 0.12 & $1.20 \mathrm{E}-03$ & $5.00 \mathrm{E}-07$ & $1.20 \mathrm{E}-03$ & 0.037 \\
\hline$T=111$ & $4.43 \mathrm{E}+06$ & 3-Ply + 3/4 Mortar & 38.1 & 0.9525 & 1.905 & 404 & $3.80 \mathrm{E}-03$ & $5.00 \mathrm{E}-07$ & $3.80 \mathrm{E}-03$ & 0.12 & $1.20 \mathrm{E}-03$ & $5.00 \mathrm{E}-07$ & $1.20 \mathrm{E}-03$ & 0.037 \\
\hline $\mathrm{T}-112$ & $4.43 E+06$ & 3-Ply + 3/4 Mortar & 38.1 & 0.9525 & 1.905 & 404 & $3.80 \mathrm{E}-03$ & $5.00 \mathrm{E}-07$ & $3.80 \mathrm{E}-03$ & 0.12 & $1.20 \mathrm{E}-03$ & $5.00 \mathrm{E}-07$ & $1.20 \mathrm{E}-03$ & 0.037 \\
\hline T-201 & $1.46 \mathrm{E}+05$ & Hardener + 3-Ply & 30.48 & 0.9525 & 0.000 & 335 & $3.80 \mathrm{E}-03$ & $5.00 \mathrm{E}-07$ & $3.80 \mathrm{E}-03$ & 0.12 & $1.20 \mathrm{E}-03$ & $5.00 \mathrm{E}-07$ & $1.20 \mathrm{E}-03$ & 0.037 \\
\hline T-202 & $1.46 \mathrm{E}+05$ & Hardener + 3-Ply & 30.48 & 0.9525 & 0.000 & 335 & $3.80 \mathrm{E}-03$ & $5.00 \mathrm{E}-07$ & $3.80 \mathrm{E}-03$ & 0.12 & $1.20 \mathrm{E}-03$ & $5.00 \mathrm{E}-07$ & $1.20 \mathrm{E}-03$ & 0.037 \\
\hline T-203 & $1.46 \mathrm{E}+05$ & Hardener + 3-Ply & 30.48 & 0.9525 & 0.000 & 335 & $3.80 \mathrm{E}-03$ & $5.00 \mathrm{E}-07$ & $3.80 \mathrm{E}-03$ & 0.12 & $1.20 \mathrm{E}-03$ & $5.00 \mathrm{E}-07$ & $1.20 \mathrm{E}-03$ & 0.037 \\
\hline $\mathrm{T}-204$ & $1.46 \mathrm{E}+05$ & Hardener + 3-Ply & 30.48 & 0.9525 & 0.000 & 335 & $3.80 \mathrm{E}-03$ & $5.00 \mathrm{E}-07$ & $3.80 \mathrm{E}-03$ & 0.12 & $1.20 \mathrm{E}-03$ & $5.00 \mathrm{E}-07$ & $1.20 \mathrm{E}-03$ & 0.037 \\
\hline TX-101 & $4.38 \mathrm{E}+06$ & Hardener + 3-Ply $+3 / 4$ Gunite & 38.1 & 0.9525 & 1.905 & 427 & $3.80 \mathrm{E}-03$ & $5.00 \mathrm{E}-07$ & $3.80 \mathrm{E}-03$ & 0.12 & $1.20 \mathrm{E}-03$ & $5.00 \mathrm{E}-07$ & $1.20 \mathrm{E}-03$ & 0.037 \\
\hline TX-102 & $4.38 \mathrm{E}+06$ & Hardener + 3-Ply + 3/4 Gunite & 38.1 & 0.9525 & 1.905 & 427 & $3.80 \mathrm{E}-03$ & $5.00 \mathrm{E}-07$ & $3.80 \mathrm{E}-03$ & 0.12 & $1.20 \mathrm{E}-03$ & $5.00 \mathrm{E}-07$ & $1.20 \mathrm{E}-03$ & 0.037 \\
\hline TX-103 & $4.38 \mathrm{E}+06$ & Hardener + 3-Ply + $3 / 4$ Gunite & 38.1 & 0.9525 & 1.905 & 427 & $3.80 \mathrm{E}-03$ & $5.00 \mathrm{E}-07$ & $3.80 \mathrm{E}-03$ & 0.12 & $1.20 \mathrm{E}-03$ & $5.00 \mathrm{E}-07$ & $1.20 \mathrm{E}-03$ & 0.037 \\
\hline TX-104 & $4.38 \mathrm{E}+06$ & Hardener + 3-Ply $+3 / 4$ Gunite & 38.1 & 0.9525 & 1.905 & 427 & $3.80 \mathrm{E}-03$ & $5.00 \mathrm{E}-07$ & $3.80 \mathrm{E}-03$ & 0.12 & $1.20 \mathrm{E}-03$ & $5.00 \mathrm{E}-07$ & $1.20 \mathrm{E}-03$ & 0.037 \\
\hline TX-105 & $4.38 \mathrm{E}+06$ & Hardener + 3-Ply + $3 / 4$ Gunite & 38.1 & 0.9525 & 1.905 & 427 & $3.80 \mathrm{E}-03$ & $5.00 \mathrm{E}-07$ & $3.80 \mathrm{E}-03$ & 0.12 & $1.20 \mathrm{E}-03$ & $5.00 \mathrm{E}-07$ & $1.20 \mathrm{E}-03$ & 0.037 \\
\hline TX-106 & $4.38 \mathrm{E}+06$ & Hardener + 3-Ply $+3 / 4$ Gunite & 38.1 & 0.9525 & 1.905 & 427 & $3.80 \mathrm{E}-03$ & $5.00 \mathrm{E}-07$ & $3.80 \mathrm{E}-03$ & 0.12 & $1.20 \mathrm{E}-03$ & $5.00 \mathrm{E}-07$ & $1.20 \mathrm{E}-03$ & 0.037 \\
\hline TX-107 & $4.38 \mathrm{E}+06$ & Hardener + 3-Ply + 3/4 Gunite & 38.1 & 0.9525 & 1.905 & 427 & $3.80 \mathrm{E}-03$ & $5.00 \mathrm{E}-07$ & $3.80 \mathrm{E}-03$ & 0.12 & $1.20 \mathrm{E}-03$ & $5.00 \mathrm{E}-07$ & $1.20 \mathrm{E}-03$ & 0.037 \\
\hline TX-108 & $4.38 \mathrm{E}+06$ & Hardener + 3-Ply + $3 / 4$ Gunite & 38.1 & 0.9525 & 1.905 & 427 & $3.80 \mathrm{E}-03$ & $5.00 \mathrm{E}-07$ & $3.80 \mathrm{E}-03$ & 0.12 & $1.20 \mathrm{E}-03$ & $5.00 \mathrm{E}-07$ & $1.20 \mathrm{E}-03$ & 0.037 \\
\hline TX-109 & $4.38 \mathrm{E}+06$ & Hardener + 3-Ply + 3/4 Gunite & 38.1 & 0.9525 & 1.905 & 427 & $3.80 \mathrm{E}-03$ & $5.00 \mathrm{E}-07$ & $3.80 \mathrm{E}-03$ & 0.12 & $1.20 \mathrm{E}-03$ & $5.00 \mathrm{E}-07$ & $1.20 \mathrm{E}-03$ & 0.037 \\
\hline TX-110 & $4.38 \mathrm{E}+06$ & Hardener + 3-Ply + $3 / 4$ Gunite & 38.1 & 0.9525 & 1.905 & 427 & $3.80 \mathrm{E}-03$ & $5.00 \mathrm{E}-07$ & $3.80 \mathrm{E}-03$ & 0.12 & $1.20 \mathrm{E}-03$ & $5.00 \mathrm{E}-07$ & $1.20 \mathrm{E}-03$ & 0.037 \\
\hline TX-111 & $4.38 \mathrm{E}+06$ & Hardener + 3-Ply + 3/4 Gunite & 38.1 & 0.9525 & 1.905 & 427 & $3.80 \mathrm{E}-03$ & $5.00 \mathrm{E}-07$ & $3.80 \mathrm{E}-03$ & 0.12 & $1.20 \mathrm{E}-03$ & $5.00 \mathrm{E}-07$ & $1.20 \mathrm{E}-03$ & 0.037 \\
\hline TX-112 & $4.38 \mathrm{E}+06$ & Hardener + 3-Ply $+3 / 4$ Gunite & 38.1 & 0.9525 & 1.905 & 427 & $3.80 \mathrm{E}-03$ & $5.00 \mathrm{E}-07$ & $3.80 \mathrm{E}-03$ & 0.12 & $1.20 \mathrm{E}-03$ & $5.00 \mathrm{E}-07$ & $1.20 \mathrm{E}-03$ & 0.037 \\
\hline TX-113 & $4.38 \mathrm{E}+06$ & Hardener + 3-Ply + 3/4 Gunite & 38.1 & 0.9525 & 1.905 & 427 & $3.80 \mathrm{E}-03$ & $5.00 \mathrm{E}-07$ & $3.80 \mathrm{E}-03$ & 0.12 & $1.20 \mathrm{E}-03$ & $5.00 \mathrm{E}-07$ & $1.20 \mathrm{E}-03$ & 0.037 \\
\hline TX-114 & $4.38 \mathrm{E}+06$ & Hardener + 3-Ply + 3/4 Gunite & 38.1 & 0.9525 & 1.905 & 427 & $3.80 \mathrm{E}-03$ & $5.00 \mathrm{E}-07$ & $3.80 \mathrm{E}-03$ & 0.12 & $1.20 \mathrm{E}-03$ & $5.00 \mathrm{E}-07$ & $1.20 \mathrm{E}-03$ & 0.037 \\
\hline TX-115 & $4.38 \mathrm{E}+06$ & Hardener + 3-Ply + 3/4 Gunite & 38.1 & 0.9525 & 1.905 & 427 & $3.80 \mathrm{E}-03$ & $5.00 \mathrm{E}-07$ & $3.80 \mathrm{E}-03$ & 0.12 & $1.20 \mathrm{E}-03$ & $5.00 \mathrm{E}-07$ & $1.20 \mathrm{E}-03$ & 0.037 \\
\hline TX-116 & $4.38 \mathrm{E}+06$ & Hardener + 3-Ply + 3/4 Gunite & 38.1 & 0.9525 & 1.905 & 427 & $3.80 \mathrm{E}-03$ & $5.00 \mathrm{E}-07$ & $3.80 \mathrm{E}-03$ & 0.12 & $1.20 \mathrm{E}-03$ & $5.00 \mathrm{E}-07$ & $1.20 \mathrm{E}-03$ & 0.037 \\
\hline
\end{tabular}




\section{RPP-5926 REV 9}

Table B-1. The Thickness of Dome Coatings for 149 Single-Shell Tanks and Diffusion Coefficients for Hydrogen and Methane.

\begin{tabular}{|c|c|c|c|c|c|c|c|c|c|c|c|c|c|c|}
\hline Tank & $\begin{array}{l}\text { Dome } \\
\text { Area } \\
\left(\mathrm{cm}^{2}\right)\end{array}$ & Tank Dome Coatings & $\begin{array}{c}\text { Concrete } \\
\text { Thick } \\
\text { (cm) }\end{array}$ & $\begin{array}{c}\text { Asphaltic } \\
\text { Thick } \\
\text { (cm) }\end{array}$ & $\begin{array}{l}\text { Gunite } \\
\text { Thick } \\
\text { (cm) }\end{array}$ & $\begin{array}{l}\text { Soil } \\
\text { Thick } \\
\text { (cm) }\end{array}$ & $\begin{array}{l}\text { Concrete } \\
\text { De of } \mathrm{H}_{2} \\
\left(\mathrm{~cm}^{2} / \mathrm{sec}\right)\end{array}$ & $\begin{array}{l}\text { Asphaltic } \\
\text { De of } \mathrm{H}_{2} \\
\left(\mathrm{~cm}^{2} / \mathrm{sec}\right)\end{array}$ & $\begin{array}{c}\text { Gunite } \\
\text { De of } \mathrm{H}_{2} \\
\left(\mathrm{~cm}^{2} / \mathrm{sec}\right)\end{array}$ & \begin{tabular}{|c|} 
Soil \\
De of $\mathrm{H}_{2}$ \\
$\left(\mathrm{~cm}^{2} / \mathrm{sec}\right)$
\end{tabular} & $\begin{array}{c}\text { Concrete De } \\
\text { of CH. } \\
\left(\mathrm{cm}^{2} / \mathrm{sec}\right)\end{array}$ & $\begin{array}{c}\text { Asphaltic } \\
\text { De of CH4 } \\
\left(\mathrm{cm}^{2} / \mathrm{sec}\right)\end{array}$ & $\begin{array}{c}\text { Gunite De } \\
\text { of } \mathrm{CH}_{4} \\
\left(\mathrm{~cm}^{2} / \mathrm{sec}\right)\end{array}$ & $\begin{array}{c}\text { Soil } \\
\text { De of } \mathrm{CH}_{4} \\
\left(\mathrm{~cm}^{2} / \mathrm{sec}\right)\end{array}$ \\
\hline TX-117 & $4.38 \mathrm{E}+06$ & Hardener + 3-Ply + 3/4 Gunite & 38.1 & 0.9525 & 1.905 & 427 & $3.80 \mathrm{E}-03$ & $5.00 \mathrm{E}-07$ & $3.80 \mathrm{E}-03$ & 0.12 & $1.20 \mathrm{E}-03$ & $5.00 \mathrm{E}-07$ & \begin{tabular}{|l|}
$1.20 \mathrm{E}-03$ \\
\end{tabular} & 0.037 \\
\hline $\mathrm{TX}-118$ & $4.38 \mathrm{E}+06$ & Hardener + 3-Ply + 3/4 Gunite & 38.1 & 0.9525 & 1.905 & 427 & $3.80 \mathrm{E}-03$ & $5.00 \mathrm{E}-07$ & $3.80 \mathrm{E}-03$ & 0.12 & $1.20 \mathrm{E}-03$ & $5.00 \mathrm{E}-07$ & $1.20 \mathrm{E}-03$ & 0.037 \\
\hline TY-101 & $4.38 \mathrm{E}+06$ & Hardener + 3-Ply $+3 / 4$ Gunite & 38.1 & 0.9525 & 1.905 & 427 & $3.80 \mathrm{E}-03$ & $5.00 \mathrm{E}-07$ & $3.80 \mathrm{E}-03$ & 0.12 & $1.20 \mathrm{E}-03$ & $5.00 \mathrm{E}-07$ & $1.20 \mathrm{E}-03$ & 0.037 \\
\hline TY-102 & $4.38 \mathrm{E}+06$ & Hardener $+3-\mathrm{Ply}+3 / 4$ Gunite & 38.1 & 0.9525 & 1.905 & 427 & $3.80 \mathrm{E}-03$ & $5.00 \mathrm{E}-07$ & $3.80 \mathrm{E}-03$ & 0.12 & $1.20 \mathrm{E}-03$ & $5.00 \mathrm{E}-07$ & $1.20 \mathrm{E}-03$ & 0.037 \\
\hline TY-103 & $4.38 \mathrm{E}+06$ & Hardener $+3-\mathrm{Ply}+3 / 4$ Gunite & 38.1 & 0.9525 & 1.905 & 427 & $3.80 \mathrm{E}-03$ & $5.00 \mathrm{E}-07$ & $3.80 \mathrm{E}-03$ & 0.12 & $1.20 \mathrm{E}-03$ & $5.00 \mathrm{E}-07$ & $1.20 \mathrm{E}-03$ & 0.037 \\
\hline TY-104 & $4.38 \mathrm{E}+06$ & Hardener + 3-Ply + 3/4 Gunite & 38.1 & 0.9525 & 1.905 & 427 & $3.80 \mathrm{E}-03$ & $5.00 \mathrm{E}-07$ & $3.80 \mathrm{E}-03$ & 0.12 & $1.20 \mathrm{E}-03$ & $5.00 \mathrm{E}-07$ & $1.20 \mathrm{E}-03$ & 0.037 \\
\hline TY-105 & $4.38 \mathrm{E}+06$ & Hardener + 3-Ply + 3/4 Gunite & 38.1 & 0.9525 & 1.905 & 427 & $3.80 \mathrm{E}-03$ & $5.00 \mathrm{E}-07$ & $3.80 \mathrm{E}-03$ & 0.12 & $1.20 \mathrm{E}-03$ & $5.00 \mathrm{E}-07$ & $1.20 \mathrm{E}-03$ & 0.037 \\
\hline TY-106 & $4.38 \mathrm{E}+06$ & Hardener + 3-Ply + 3/4 Gunite & 38.1 & 0.9525 & 1.905 & 427 & $3.80 \mathrm{E}-03$ & $5.00 \mathrm{E}-07$ & $3.80 \mathrm{E}-03$ & 0.12 & $1.20 \mathrm{E}-03$ & $5.00 \mathrm{E}-07$ & $1.20 \mathrm{E}-03$ & 0.037 \\
\hline U-101 & $4.43 \mathrm{E}+06$ & $3-$ Ply $+3 / 4$ Mortar & 38.1 & 0.9525 & 1.905 & 404 & $3.80 \mathrm{E}-03$ & $5.00 \mathrm{E}-07$ & $3.80 \mathrm{E}-03$ & 0.12 & $1.20 \mathrm{E}-03$ & $5.00 \mathrm{E}-07$ & $1.20 \mathrm{E}-03$ & 0.037 \\
\hline U-102 & $4.43 \mathrm{E}+06$ & 3-Ply + 3/4 Mortar & 38.1 & 0.9525 & 1.905 & 404 & $3.80 \mathrm{E}-03$ & $5.00 \mathrm{E}-07$ & $3.80 \mathrm{E}-03$ & 0.12 & $1.20 \mathrm{E}-03$ & $5.00 \mathrm{E}-07$ & $1.20 \mathrm{E}-03$ & 0.037 \\
\hline U-103 & $4.43 \mathrm{E}+06$ & 3-Ply + 3/4 Mortar & 38.1 & 0.9525 & 1.905 & 404 & $3.80 \mathrm{E}-03$ & $5.00 \mathrm{E}-07$ & $3.80 \mathrm{E}-03$ & 0.12 & $1.20 \mathrm{E}-03$ & $5.00 \mathrm{E}-07$ & $1.20 \mathrm{E}-03$ & 0.037 \\
\hline U-104 & $4.43 \mathrm{E}+06$ & 3-Ply + 3/4 Mortar & 38.1 & 0.9525 & 1.905 & 404 & $3.80 \mathrm{E}-03$ & $5.00 \mathrm{E}-07$ & $3.80 \mathrm{E}-03$ & 0.12 & $1.20 \mathrm{E}-03$ & $5.00 \mathrm{E}-07$ & $1.20 \mathrm{E}-03$ & 0.037 \\
\hline U-105 & $4.43 E+06$ & 3-Ply $+3 / 4$ Mortar & 38.1 & 0.9525 & 1.905 & 404 & $3.80 \mathrm{E}-03$ & $5.00 \mathrm{E}-07$ & $3.80 \mathrm{E}-03$ & 0.12 & $1.20 \mathrm{E}-03$ & $5.00 \mathrm{E}-07$ & $1.20 \mathrm{E}-03$ & 0.037 \\
\hline U-106 & $4.43 \mathrm{E}+06$ & 3-Ply + 3/4 Mortar & 38.1 & 0.9525 & 1.905 & 404 & $3.80 \mathrm{E}-03$ & $5.00 \mathrm{E}-07$ & $3.80 \mathrm{E}-03$ & 0.12 & $1.20 \mathrm{E}-03$ & $5.00 \mathrm{E}-07$ & $1.20 \mathrm{E}-03$ & 0.037 \\
\hline U-107 & $4.43 E+06$ & 3-Ply + 3/4 Mortar & 38.1 & 0.9525 & 1.905 & 404 & $3.80 \mathrm{E}-03$ & $5.00 \mathrm{E}-07$ & $3.80 \mathrm{E}-03$ & 0.12 & $1.20 \mathrm{E}-03$ & $5.00 \mathrm{E}-07$ & $1.20 \mathrm{E}-03$ & 0.037 \\
\hline U-108 & $4.43 E+06$ & 3-Ply + 3/4 Mortar & 38.1 & 0.9525 & 1.905 & 404 & $3.80 \mathrm{E}-03$ & $5.00 \mathrm{E}-07$ & $3.80 \mathrm{E}-03$ & 0.12 & $1.20 \mathrm{E}-03$ & $5.00 \mathrm{E}-07$ & $1.20 \mathrm{E}-03$ & 0.037 \\
\hline U-109 & $4.43 \mathrm{E}+06$ & 3-Ply + 3/4 Mortar & 38.1 & 0.9525 & 1.905 & 404 & $3.80 \mathrm{E}-03$ & $5.00 \mathrm{E}-07$ & $3.80 \mathrm{E}-03$ & 0.12 & $1.20 \mathrm{E}-03$ & $5.00 \mathrm{E}-07$ & $1.20 \mathrm{E}-03$ & 0.037 \\
\hline U-110 & $4.43 \mathrm{E}+06$ & 3-Ply + 3/4 Mortar & 38.1 & 0.9525 & 1.905 & 404 & $3.80 \mathrm{E}-03$ & \begin{tabular}{|l|}
$5.00 \mathrm{E}-07$ \\
\end{tabular} & $3.80 \mathrm{E}-03$ & 0.12 & $1.20 \mathrm{E}-03$ & $5.00 \mathrm{E}-07$ & $1.20 \mathrm{E}-03$ & 0.037 \\
\hline U-111 & $4.43 \mathrm{E}+06$ & 3-Ply + 3/4 Mortar & 38.1 & 0.9525 & 1.905 & 404 & $3.80 \mathrm{E}-03$ & $5.00 \mathrm{E}-07$ & $3.80 \mathrm{E}-03$ & 0.12 & $1.20 \mathrm{E}-03$ & $5.00 \mathrm{E}-07$ & $1.20 \mathrm{E}-03$ & 0.037 \\
\hline U-112 & $4.43 E+06$ & 3-Ply + 3/4 Mortar & 38.1 & 0.9525 & 1.905 & 404 & $3.80 \mathrm{E}-03$ & $5.00 \mathrm{E}-07$ & $3.80 \mathrm{E}-03$ & 0.12 & $1.20 \mathrm{E}-03$ & $5.00 \mathrm{E}-07$ & $1.20 \mathrm{E}-03$ & 0.037 \\
\hline U-201 & $1.46 \mathrm{E}+05$ & Hardener + 3-Ply & 30.48 & 0.9525 & 0.000 & 335 & $3.80 \mathrm{E}-03$ & $5.00 \mathrm{E}-07$ & $3.80 \mathrm{E}-03$ & 0.12 & $1.20 \mathrm{E}-03$ & $5.00 \mathrm{E}-07$ & $1.20 \mathrm{E}-03$ & 0.037 \\
\hline U-202 & $1.46 \mathrm{E}+05$ & Hardener + 3-Ply & 30.48 & 0.9525 & 0.000 & 335 & $3.80 \mathrm{E}-03$ & $5.00 \mathrm{E}-07$ & $3.80 \mathrm{E}-03$ & 0.12 & $1.20 \mathrm{E}-03$ & $5.00 \mathrm{E}-07$ & \begin{tabular}{|l|}
$1.20 \mathrm{E}-03$ \\
\end{tabular} & 0.037 \\
\hline U-203 & $1.46 \mathrm{E}+05$ & Hardener + 3-Ply & 30.48 & 0.9525 & 0.000 & 335 & $3.80 \mathrm{E}-03$ & $5.00 \mathrm{E}-07$ & $3.80 \mathrm{E}-03$ & 0.12 & $1.20 \mathrm{E}-03$ & $5.00 \mathrm{E}-07$ & $1.20 \mathrm{E}-03$ & 0.037 \\
\hline U-204 & $1.46 \mathrm{E}+05$ & Hardener + 3-Ply & 30.48 & 0.9525 & 0.000 & 335 & $3.80 \mathrm{E}-03$ & $5.00 \mathrm{E}-07$ & $3.80 \mathrm{E}-03$ & 0.12 & $1.20 \mathrm{E}-03$ & $5.00 \mathrm{E}-07$ & $1.20 \mathrm{E}-03$ & 0.037 \\
\hline
\end{tabular}


Table B-2. The Diffusion Resistance and Gas Diffusion Rates of Hydrogen and Methane for 149 Single-Shell Tanks.

\begin{tabular}{|c|c|c|c|c|c|c|c|c|c|c|c|c|}
\hline Tank & $\begin{array}{c}\text { Concrete } \\
\text { Resist of } \mathbf{H}_{2} \\
\text { (sec/cm) }\end{array}$ & $\begin{array}{l}\text { Asphaltic } \\
\text { Resist of } \mathrm{H}_{2} \\
(\mathrm{sec} / \mathrm{cm})\end{array}$ & $\begin{array}{c}\text { Gunite } \\
\text { Resist of } \mathrm{H}_{2} \\
(\mathrm{sec} / \mathrm{cm})\end{array}$ & $\begin{array}{c}\text { Soil } \\
\text { Resist of } \mathbf{H}_{2} \\
\text { (sec/cm) }\end{array}$ & $\begin{array}{c}\text { Concrete } \\
\text { Resist of CH } \\
\text { (sec/cm) }\end{array}$ & $\begin{array}{c}\text { Asphaltic } \\
\text { Resist of } \mathrm{CH}_{4} \\
\text { (sec/cm) }\end{array}$ & $\begin{array}{c}\text { Gunite } \\
\text { Resist of } \mathrm{CH}_{4} \\
\text { (sec/cm) }\end{array}$ & $\begin{array}{c}\text { Soil } \\
\text { Resist of CH } \\
(\mathrm{sec} / \mathrm{cm})\end{array}$ & $\begin{array}{c}\text { Total } \\
\text { Resist of } \mathbf{H}_{2} \\
(\mathrm{sec} / \mathrm{cm})\end{array}$ & \begin{tabular}{|c|}
$\begin{array}{c}\text { Total } \\
\text { Resist of } \mathrm{CH}_{4} \\
\text { (sec/cm) }\end{array}$ \\
\end{tabular} & \begin{tabular}{|c|}
$\mathbf{H}_{2}$ Diff Rate \\
$\begin{array}{c}\mathbf{A i} / \mathbf{R i} \\
(\mathbf{c f m})\end{array}$ \\
\end{tabular} & $\begin{array}{c}\mathbf{C H}_{4} \text { Diff Rate } \\
\mathbf{A i} / \mathbf{R i} \\
(\mathrm{cfm})\end{array}$ \\
\hline A-101 & $1.00 \mathrm{E}+04$ & $1.27 \mathrm{E}+06$ & $0.00 \mathrm{E}+00$ & $3.05 \mathrm{E}+03$ & $3.18 \mathrm{E}+04$ & $1.27 \mathrm{E}+06$ & $0.00 \mathrm{E}+00$ & $9.89 \mathrm{E}+03$ & $1.28 \mathrm{E}+06$ & $1.31 \mathrm{E}+06$ & $7.25 \mathrm{E}-03$ & $7.09 \mathrm{E}-03$ \\
\hline A-102 & $1.00 \mathrm{E}+04$ & $1.27 \mathrm{E}+06$ & $0.00 \mathrm{E}+00$ & $3.05 E+03$ & $3.18 \mathrm{E}+04$ & $1.27 \mathrm{E}+06$ & $0.00 \mathrm{E}+00$ & $9.89 \mathrm{E}+03$ & $1.28 \mathrm{E}+06$ & $1.31 \mathrm{E}+06$ & $7.25 \mathrm{E}-03$ & $7.09 \mathrm{E}-03$ \\
\hline A-103 & $1.00 \mathrm{E}+04$ & $1.27 \mathrm{E}+06$ & $0.00 \mathrm{E}+00$ & $3.05 \mathrm{E}+03$ & $3.18 \mathrm{E}+04$ & $1.27 \mathrm{E}+06$ & $0.00 \mathrm{E}+00$ & $9.89 \mathrm{E}+03$ & $1.28 \mathrm{E}+06$ & $1.31 \mathrm{E}+06$ & $7.25 \mathrm{E}-03$ & $7.09 \mathrm{E}-03$ \\
\hline A-104 & $1.00 \mathrm{E}+04$ & $1.27 \mathrm{E}+06$ & $0.00 \mathrm{E}+00$ & $3.05 \mathrm{E}+03$ & $3.18 \mathrm{E}+04$ & $1.27 \mathrm{E}+06$ & $0.00 \mathrm{E}+00$ & $9.89 \mathrm{E}+03$ & $1.28 \mathrm{E}+06$ & $1.31 \mathrm{E}+06$ & $7.25 \mathrm{E}-03$ & $7.09 \mathrm{E}-03$ \\
\hline A-105 & $1.00 \mathrm{E}+04$ & $1.27 \mathrm{E}+06$ & $0.00 \mathrm{E}+00$ & $3.05 \mathrm{E}+03$ & $3.18 \mathrm{E}+04$ & $1.27 \mathrm{E}+06$ & $0.00 \mathrm{E}+00$ & $9.89 \mathrm{E}+03$ & $1.28 \mathrm{E}+06$ & $1.31 \mathrm{E}+06$ & $7.25 \mathrm{E}-03$ & $7.09 \mathrm{E}-03$ \\
\hline A-106 & $1.00 \mathrm{E}+04$ & $1.27 \mathrm{E}+06$ & $0.00 \mathrm{E}+00$ & $3.05 \mathrm{E}+03$ & $3.18 \mathrm{E}+04$ & $1.27 \mathrm{E}+06$ & $0.00 \mathrm{E}+00$ & $9.89 \mathrm{E}+03$ & $1.28 \mathrm{E}+06$ & $1.31 \mathrm{E}+06$ & $7.25 \mathrm{E}-03$ & $7.09 \mathrm{E}-03$ \\
\hline $\mathrm{AX}-101$ & $1.00 \mathrm{E}+04$ & $0.00 \mathrm{E}+00$ & $0.00 \mathrm{E}+00$ & $3.05 \mathrm{E}+03$ & $3.18 \mathrm{E}+04$ & $0.00 \mathrm{E}+00$ & $0.00 \mathrm{E}+00$ & $9.89 \mathrm{E}+03$ & $1.31 \mathrm{E}+04$ & $4.16 \mathrm{E}+04$ & $7.11 \mathrm{E}-01$ & $2.23 \mathrm{E}-01$ \\
\hline $\mathrm{AX}-102$ & $1.00 \mathrm{E}+04$ & $0.00 \mathrm{E}+00$ & $0.00 \mathrm{E}+00$ & $3.05 \mathrm{E}+03$ & $3.18 \mathrm{E}+04$ & $0.00 \mathrm{E}+00$ & $0.00 \mathrm{E}+00$ & $9.89 \mathrm{E}+03$ & $1.31 \mathrm{E}+04$ & $4.16 \mathrm{E}+04$ & $7.11 \mathrm{E}-01$ & $2.23 \mathrm{E}-01$ \\
\hline AX-103 & $1.00 \mathrm{E}+04$ & $0.00 \mathrm{E}+00$ & $0.00 \mathrm{E}+00$ & $3.05 \mathrm{E}+03$ & $3.18 \mathrm{E}+04$ & $0.00 \mathrm{E}+00$ & $0.00 \mathrm{E}+00$ & $9.89 \mathrm{E}+03$ & $1.31 \mathrm{E}+04$ & $4.16 \mathrm{E}+04$ & $7.11 \mathrm{E}-01$ & $2.23 \mathrm{E}-01$ \\
\hline $\mathrm{AX}-104$ & $1.00 \mathrm{E}+04$ & $0.00 \mathrm{E}+00$ & $0.00 \mathrm{E}+00$ & $3.05 \mathrm{E}+03$ & $3.18 \mathrm{E}+04$ & $0.00 \mathrm{E}+00$ & $0.00 \mathrm{E}+00$ & $9.89 \mathrm{E}+03$ & $1.31 \mathrm{E}+04$ & $4.16 \mathrm{E}+04$ & $7.11 \mathrm{E}-01$ & $2.23 \mathrm{E}-01$ \\
\hline B-101 & $1.00 \mathrm{E}+04$ & $1.91 \mathrm{E}+06$ & $5.01 E+02$ & $3.37 \mathrm{E}+03$ & $3.18 \mathrm{E}+04$ & $1.91 \mathrm{E}+06$ & $1.59 \mathrm{E}+03$ & $1.09 \mathrm{E}+04$ & $1.92 \mathrm{E}+06$ & $1.95 \mathrm{E}+06$ & $4.89 \mathrm{E}-03$ & $4.82 \mathrm{E}-03$ \\
\hline B-102 & $1.00 \mathrm{E}+04$ & $1.91 E+06$ & $5.01 E+02$ & $3.37 \mathrm{E}+03$ & $3.18 \mathrm{E}+04$ & $1.91 \mathrm{E}+06$ & $1.59 \mathrm{E}+03$ & $1.09 \mathrm{E}+04$ & $1.92 \mathrm{E}+06$ & $1.95 \mathrm{E}+06$ & $4.89 \mathrm{E}-03$ & $4.82 \mathrm{E}-03$ \\
\hline B-103 & $1.00 \mathrm{E}+04$ & $1.91 E+06$ & $5.01 E+02$ & $3.37 \mathrm{E}+03$ & $3.18 \mathrm{E}+04$ & $1.91 \mathrm{E}+06$ & $1.59 \mathrm{E}+03$ & $1.09 \mathrm{E}+04$ & $1.92 \mathrm{E}+06$ & $1.95 \mathrm{E}+06$ & $4.89 \mathrm{E}-03$ & $4.82 \mathrm{E}-03$ \\
\hline B-104 & $1.00 \mathrm{E}+04$ & $1.91 \mathrm{E}+06$ & $5.01 \mathrm{E}+02$ & $3.37 \mathrm{E}+03$ & $3.18 \mathrm{E}+04$ & $1.91 \mathrm{E}+06$ & $1.59 \mathrm{E}+03$ & $1.09 \mathrm{E}+04$ & $1.92 \mathrm{E}+06$ & $1.95 \mathrm{E}+06$ & $4.89 \mathrm{E}-03$ & $4.82 \mathrm{E}-03$ \\
\hline B-105 & $1.00 \mathrm{E}+04$ & $1.91 \mathrm{E}+06$ & $5.01 \mathrm{E}+02$ & $3.37 \mathrm{E}+03$ & $3.18 \mathrm{E}+04$ & $1.91 \mathrm{E}+06$ & $1.59 \mathrm{E}+03$ & $1.09 \mathrm{E}+04$ & $1.92 \mathrm{E}+06$ & $1.95 \mathrm{E}+06$ & $4.89 \mathrm{E}-03$ & $4.82 \mathrm{E}-03$ \\
\hline B-106 & $1.00 \mathrm{E}+04$ & $1.91 \mathrm{E}+06$ & $5.01 \mathrm{E}+02$ & $3.37 \mathrm{E}+03$ & $3.18 \mathrm{E}+04$ & $1.91 \mathrm{E}+06$ & $1.59 \mathrm{E}+03$ & $1.09 \mathrm{E}+04$ & $1.92 \mathrm{E}+06$ & $1.95 \mathrm{E}+06$ & $4.89 \mathrm{E}-03$ & $4.82 \mathrm{E}-03$ \\
\hline B-107 & $1.00 \mathrm{E}+04$ & $1.91 \mathrm{E}+06$ & $5.01 \mathrm{E}+02$ & $3.37 \mathrm{E}+03$ & $3.18 \mathrm{E}+04$ & $1.91 \mathrm{E}+06$ & $1.59 \mathrm{E}+03$ & $1.09 \mathrm{E}+04$ & $1.92 \mathrm{E}+06$ & $1.95 \mathrm{E}+06$ & $4.89 \mathrm{E}-03$ & $4.82 \mathrm{E}-03$ \\
\hline B-108 & $1.00 \mathrm{E}+04$ & $1.91 \mathrm{E}+06$ & $5.01 \mathrm{E}+02$ & $3.37 \mathrm{E}+03$ & $3.18 \mathrm{E}+04$ & $1.91 E+06$ & $1.59 \mathrm{E}+03$ & $1.09 \mathrm{E}+04$ & $1.92 \mathrm{E}+06$ & $1.95 \mathrm{E}+06$ & $4.89 \mathrm{E}-03$ & $4.82 \mathrm{E}-03$ \\
\hline B-109 & $1.00 \mathrm{E}+04$ & $1.91 \mathrm{E}+06$ & $5.01 \mathrm{E}+02$ & $3.37 \mathrm{E}+03$ & $3.18 \mathrm{E}+04$ & $1.91 \mathrm{E}+06$ & $1.59 \mathrm{E}+03$ & $1.09 \mathrm{E}+04$ & $1.92 \mathrm{E}+06$ & $1.95 \mathrm{E}+06$ & $4.89 \mathrm{E}-03$ & $4.82 \mathrm{E}-03$ \\
\hline B-110 & $1.00 \mathrm{E}+04$ & $1.91 \mathrm{E}+06$ & $5.01 \mathrm{E}+02$ & $3.37 \mathrm{E}+03$ & $3.18 \mathrm{E}+04$ & $1.91 \mathrm{E}+06$ & $1.59 \mathrm{E}+03$ & $1.09 \mathrm{E}+04$ & $1.92 \mathrm{E}+06$ & $1.95 \mathrm{E}+06$ & $4.89 \mathrm{E}-03$ & $4.82 \mathrm{E}-03$ \\
\hline B-111 & $1.00 \mathrm{E}+04$ & $1.91 \mathrm{E}+06$ & $5.01 \mathrm{E}+02$ & $3.37 \mathrm{E}+03$ & $3.18 \mathrm{E}+04$ & $1.91 \mathrm{E}+06$ & $1.59 \mathrm{E}+03$ & $1.09 \mathrm{E}+04$ & $1.92 \mathrm{E}+06$ & $1.95 \mathrm{E}+06$ & $4.89 \mathrm{E}-03$ & $4.82 \mathrm{E}-03$ \\
\hline B-112 & $1.00 \mathrm{E}+04$ & $1.91 \mathrm{E}+06$ & $5.01 \mathrm{E}+02$ & $3.37 \mathrm{E}+03$ & $3.18 \mathrm{E}+04$ & $1.91 \mathrm{E}+06$ & $1.59 \mathrm{E}+03$ & $1.09 \mathrm{E}+04$ & $1.92 \mathrm{E}+06$ & $1.95 \mathrm{E}+06$ & $4.89 \mathrm{E}-03$ & $4.82 \mathrm{E}-03$ \\
\hline B-201 & $8.02 \mathrm{E}+03$ & $1.91 \mathrm{E}+06$ & $0.00 \mathrm{E}+00$ & $2.79 \mathrm{E}+03$ & $2.54 \mathrm{E}+04$ & $1.91 \mathrm{E}+06$ & $0.00 \mathrm{E}+00$ & $9.05 \mathrm{E}+03$ & $1.92 \mathrm{E}+06$ & $1.94 \mathrm{E}+06$ & $1.61 \mathrm{E}-04$ & $1.60 \mathrm{E}-04$ \\
\hline B-202 & $8.02 \mathrm{E}+03$ & $1.91 \mathrm{E}+06$ & $0.00 \mathrm{E}+00$ & $2.79 \mathrm{E}+03$ & $2.54 \mathrm{E}+04$ & $1.91 \mathrm{E}+06$ & $0.00 \mathrm{E}+00$ & $9.05 \mathrm{E}+03$ & $1.92 \mathrm{E}+06$ & $1.94 \mathrm{E}+06$ & $1.61 \mathrm{E}-04$ & $1.60 \mathrm{E}-04$ \\
\hline B-203 & $8.02 \mathrm{E}+03$ & $1.91 \mathrm{E}+06$ & $0.00 \mathrm{E}+00$ & $2.79 \mathrm{E}+03$ & $2.54 \mathrm{E}+04$ & $1.91 \mathrm{E}+06$ & $0.00 \mathrm{E}+00$ & $9.05 \mathrm{E}+03$ & $1.92 \mathrm{E}+06$ & $1.94 \mathrm{E}+06$ & $1.61 \mathrm{E}-04$ & $1.60 \mathrm{E}-04$ \\
\hline B-204 & $8.02 \mathrm{E}+03$ & $1.91 \mathrm{E}+06$ & $0.00 \mathrm{E}+00$ & $2.79 \mathrm{E}+03$ & $2.54 \mathrm{E}+04$ & $1.91 \mathrm{E}+06$ & $0.00 \mathrm{E}+00$ & $9.05 \mathrm{E}+03$ & $1.92 \mathrm{E}+06$ & $1.94 \mathrm{E}+06$ & $1.61 \mathrm{E}-04$ & $1.60 \mathrm{E}-04$ \\
\hline BX-101 & $1.00 \mathrm{E}+04$ & $1.91 \mathrm{E}+06$ & $5.01 \mathrm{E}+02$ & $3.37 \mathrm{E}+03$ & $3.18 \mathrm{E}+04$ & $1.91 \mathrm{E}+06$ & $1.59 \mathrm{E}+03$ & $1.09 \mathrm{E}+04$ & $1.92 \mathrm{E}+06$ & $1.95 \mathrm{E}+06$ & $4.89 \mathrm{E}-03$ & $4.82 \mathrm{E}-03$ \\
\hline BX-102 & $1.00 \mathrm{E}+04$ & $1.91 \mathrm{E}+06$ & $5.01 E+02$ & $3.37 \mathrm{E}+03$ & $3.18 \mathrm{E}+04$ & $1.91 \mathrm{E}+06$ & $1.59 \mathrm{E}+03$ & $1.09 \mathrm{E}+04$ & $1.92 \mathrm{E}+06$ & $1.95 \mathrm{E}+06$ & $4.89 \mathrm{E}-03$ & $4.82 \mathrm{E}-03$ \\
\hline BX-103 & $1.00 \mathrm{E}+04$ & $1.91 \mathrm{E}+06$ & $5.01 \mathrm{E}+02$ & $3.37 \mathrm{E}+03$ & $3.18 \mathrm{E}+04$ & $1.91 \mathrm{E}+06$ & $1.59 \mathrm{E}+03$ & $1.09 \mathrm{E}+04$ & $1.92 \mathrm{E}+06$ & $1.95 \mathrm{E}+06$ & $4.89 \mathrm{E}-03$ & $4.82 \mathrm{E}-03$ \\
\hline BX-104 & $1.00 \mathrm{E}+04$ & $1.91 \mathrm{E}+06$ & $5.01 \mathrm{E}+02$ & $3.37 \mathrm{E}+03$ & $3.18 \mathrm{E}+04$ & $1.91 \mathrm{E}+06$ & $1.59 \mathrm{E}+03$ & $1.09 \mathrm{E}+04$ & $1.92 \mathrm{E}+06$ & $1.95 \mathrm{E}+06$ & $4.89 \mathrm{E}-03$ & $4.82 \mathrm{E}-03$ \\
\hline BX-105 & $1.00 \mathrm{E}+04$ & $1.91 \mathrm{E}+06$ & $5.01 \mathrm{E}+02$ & $3.37 \mathrm{E}+03$ & $3.18 \mathrm{E}+04$ & $1.91 \mathrm{E}+06$ & $1.59 \mathrm{E}+03$ & $1.09 \mathrm{E}+04$ & $1.92 \mathrm{E}+06$ & $1.95 \mathrm{E}+06$ & $4.89 \mathrm{E}-03$ & $4.82 \mathrm{E}-03$ \\
\hline BX-106 & $1.00 \mathrm{E}+04$ & $1.91 \mathrm{E}+06$ & $5.01 \mathrm{E}+02$ & $3.37 \mathrm{E}+03$ & $3.18 \mathrm{E}+04$ & $1.91 \mathrm{E}+06$ & $1.59 \mathrm{E}+03$ & $1.09 \mathrm{E}+04$ & $1.92 \mathrm{E}+06$ & $1.95 \mathrm{E}+06$ & $4.89 \mathrm{E}-03$ & $4.82 \mathrm{E}-03$ \\
\hline BX-107 & $1.00 \mathrm{E}+04$ & $1.91 \mathrm{E}+06$ & $5.01 \mathrm{E}+02$ & $3.37 \mathrm{E}+03$ & $3.18 \mathrm{E}+04$ & $1.91 \mathrm{E}+06$ & $1.59 \mathrm{E}+03$ & $1.09 \mathrm{E}+04$ & $1.92 \mathrm{E}+06$ & $1.95 \mathrm{E}+06$ & $4.89 \mathrm{E}-03$ & $4.82 \mathrm{E}-03$ \\
\hline BX-108 & $1.00 \mathrm{E}+04$ & $1.91 \mathrm{E}+06$ & $5.01 \mathrm{E}+02$ & $3.37 \mathrm{E}+03$ & $3.18 \mathrm{E}+04$ & $1.91 \mathrm{E}+06$ & $1.59 \mathrm{E}+03$ & $1.09 \mathrm{E}+04$ & $1.92 \mathrm{E}+06$ & $1.95 \mathrm{E}+06$ & $4.89 \mathrm{E}-03$ & $4.82 \mathrm{E}-03$ \\
\hline BX-109 & $1.00 \mathrm{E}+04$ & $1.91 \mathrm{E}+06$ & $5.01 \mathrm{E}+02$ & $3.37 \mathrm{E}+03$ & $3.18 \mathrm{E}+04$ & $1.91 \mathrm{E}+06$ & $1.59 \mathrm{E}+03$ & $1.09 \mathrm{E}+04$ & $1.92 \mathrm{E}+06$ & $1.95 \mathrm{E}+06$ & $4.89 \mathrm{E}-03$ & $4.82 \mathrm{E}-03$ \\
\hline BX-110 & $1.00 \mathrm{E}+04$ & $1.91 \mathrm{E}+06$ & $5.01 \mathrm{E}+02$ & $3.37 \mathrm{E}+03$ & $3.18 \mathrm{E}+04$ & $1.91 \mathrm{E}+06$ & $1.59 \mathrm{E}+03$ & $1.09 \mathrm{E}+04$ & $1.92 \mathrm{E}+06$ & $1.95 \mathrm{E}+06$ & $4.89 \mathrm{E}-03$ & $4.82 \mathrm{E}-03$ \\
\hline BX-111 & $1.00 \mathrm{E}+04$ & $1.91 \mathrm{E}+06$ & $5.01 \mathrm{E}+02$ & $3.37 \mathrm{E}+03$ & $3.18 \mathrm{E}+04$ & $1.91 \mathrm{E}+06$ & $1.59 \mathrm{E}+03$ & $1.09 \mathrm{E}+04$ & $1.92 \mathrm{E}+06$ & $1.95 \mathrm{E}+06$ & $4.89 \mathrm{E}-03$ & $4.82 \mathrm{E}-03$ \\
\hline BX-112 & $1.00 \mathrm{E}+04$ & $1.91 \mathrm{E}+06$ & $5.01 \mathrm{E}+02$ & $3.37 \mathrm{E}+03$ & $3.18 \mathrm{E}+04$ & $1.91 \mathrm{E}+06$ & $1.59 \mathrm{E}+03$ & $1.09 \mathrm{E}+04$ & $1.92 \mathrm{E}+06$ & $1.95 \mathrm{E}+06$ & $4.89 \mathrm{E}-03$ & $4.82 \mathrm{E}-03$ \\
\hline BY-101 & $1.00 \mathrm{E}+04$ & $1.91 \mathrm{E}+06$ & $5.01 \mathrm{E}+02$ & $3.56 \mathrm{E}+03$ & $3.18 \mathrm{E}+04$ & $1.91 \mathrm{E}+06$ & $1.59 \mathrm{E}+03$ & $1.15 \mathrm{E}+04$ & $1.92 \mathrm{E}+06$ & $1.95 \mathrm{E}+06$ & $4.84 \mathrm{E}-03$ & $4.76 \mathrm{E}-03$ \\
\hline BY-102 & $1.00 \mathrm{E}+04$ & $1.91 \mathrm{E}+06$ & $5.01 \mathrm{E}+02$ & $3.56 \mathrm{E}+03$ & $3.18 \mathrm{E}+04$ & $1.91 \mathrm{E}+06$ & $1.59 \mathrm{E}+03$ & $1.15 \mathrm{E}+04$ & $1.92 \mathrm{E}+06$ & $1.95 \mathrm{E}+06$ & $4.84 \mathrm{E}-03$ & $4.76 \mathrm{E}-03$ \\
\hline BY-103 & $1.00 \mathrm{E}+04$ & $1.91 \mathrm{E}+06$ & $5.01 \mathrm{E}+02$ & $3.56 \mathrm{E}+03$ & $3.18 \mathrm{E}+04$ & $1.91 \mathrm{E}+06$ & $1.59 \mathrm{E}+03$ & $1.15 \mathrm{E}+04$ & $1.92 \mathrm{E}+06$ & $1.95 \mathrm{E}+06$ & $4.84 \mathrm{E}-03$ & $4.76 \mathrm{E}-03$ \\
\hline
\end{tabular}


Table B-2. The Diffusion Resistance and Gas Diffusion Rates of Hydrogen and Methane for 149 Single-Shell Tanks.

\begin{tabular}{|c|c|c|c|c|c|c|c|c|c|c|c|c|}
\hline Tank & $\begin{array}{c}\text { Concrete } \\
\text { Resist of } \mathrm{H}_{2} \\
\text { (sec/cm) }\end{array}$ & $\begin{array}{l}\text { Asphaltic } \\
\text { Resist of } \mathbf{H}_{2} \\
\text { (sec/cm) }\end{array}$ & $\begin{array}{c}\text { Gunite } \\
\text { Resist of } \mathrm{H}_{2} \\
\text { (sec/cm) }\end{array}$ & $\begin{array}{c}\text { Soil } \\
\text { Resist of } \mathrm{H}_{2} \\
\text { (sec/cm) }\end{array}$ & $\begin{array}{c}\text { Concrete } \\
\text { Resist of CH } \\
\text { (sec/cm) }\end{array}$ & $\begin{array}{c}\text { Asphaltic } \\
\text { Resist of CH } \\
\text { (sec/cm) }\end{array}$ & $\begin{array}{c}\text { Gunite } \\
\text { Resist of CH } \\
\text { (sec/cm) }\end{array}$ & $\begin{array}{c}\text { Soil } \\
\text { Resist of } \mathrm{CH}_{4} \\
\text { (sec/cm) }\end{array}$ & $\begin{array}{c}\text { Total } \\
\text { Resist of } \mathrm{H}_{2} \\
\text { (sec/cm) }\end{array}$ & $\begin{array}{c}\begin{array}{c}\text { Total } \\
\text { Resist of CH } \\
\text { (sec/cm) }\end{array} \\
\end{array}$ & $\begin{array}{c}\mathbf{H}_{2} \text { Diff Rate } \\
\mathbf{A i} / \mathbf{R i} \\
(\mathrm{cfm})\end{array}$ & \begin{tabular}{|c} 
CH. Diff Rate \\
$\begin{array}{c}\mathbf{A i} / \mathbf{R i} \\
(\mathrm{cfm})\end{array}$ \\
\end{tabular} \\
\hline BY-104 & $1.00 \mathrm{E}+04$ & $1.91 \mathrm{E}+06$ & $5.01 \mathrm{E}+02$ & $3.56 \mathrm{E}+03$ & $3.18 \mathrm{E}+04$ & $1.91 \mathrm{E}+06$ & $1.59 \mathrm{E}+03$ & $1.15 \mathrm{E}+04$ & $1.92 \mathrm{E}+06$ & $1.95 \mathrm{E}+06$ & $4.84 \mathrm{E}-03$ & $4.76 \mathrm{E}-03$ \\
\hline BY-105 & $1.00 \mathrm{E}+04$ & $1.91 \mathrm{E}+06$ & $5.01 \mathrm{E}+02$ & $3.56 \mathrm{E}+03$ & $3.18 \mathrm{E}+04$ & $1.91 \mathrm{E}+06$ & $1.59 \mathrm{E}+03$ & $1.15 E+04$ & $1.92 \mathrm{E}+06$ & $1.95 \mathrm{E}+06$ & $4.84 \mathrm{E}-03$ & $4.76 \mathrm{E}-03$ \\
\hline BY-106 & $1.00 \mathrm{E}+04$ & $1.91 \mathrm{E}+06$ & $5.01 \mathrm{E}+02$ & $3.56 \mathrm{E}+03$ & $3.18 \mathrm{E}+04$ & $1.91 \mathrm{E}+06$ & $1.59 \mathrm{E}+03$ & $1.15 \mathrm{E}+04$ & $1.92 \mathrm{E}+06$ & $1.95 \mathrm{E}+06$ & $4.84 \mathrm{E}-03$ & $4.76 \mathrm{E}-03$ \\
\hline BY-107 & $1.00 \mathrm{E}+04$ & $1.91 \mathrm{E}+06$ & $5.01 E+02$ & $3.56 \mathrm{E}+03$ & $3.18 \mathrm{E}+04$ & $1.91 \mathrm{E}+06$ & $1.59 \mathrm{E}+03$ & $1.15 E+04$ & $1.92 \mathrm{E}+06$ & $1.95 \mathrm{E}+06$ & $4.84 \mathrm{E}-03$ & $4.76 \mathrm{E}-03$ \\
\hline BY-108 & $1.00 \mathrm{E}+04$ & $1.91 \mathrm{E}+06$ & $5.01 E+02$ & $3.56 \mathrm{E}+03$ & $3.18 \mathrm{E}+04$ & $1.91 \mathrm{E}+06$ & $1.59 \mathrm{E}+03$ & $1.15 \mathrm{E}+04$ & $1.92 \mathrm{E}+06$ & $1.95 \mathrm{E}+06$ & $4.84 \mathrm{E}-03$ & $4.76 \mathrm{E}-03$ \\
\hline BY-109 & $1.00 \mathrm{E}+04$ & $1.91 \mathrm{E}+06$ & $5.01 E+02$ & $3.56 \mathrm{E}+03$ & $3.18 \mathrm{E}+04$ & $1.91 \mathrm{E}+06$ & $1.59 \mathrm{E}+03$ & $1.15 \mathrm{E}+04$ & $1.92 \mathrm{E}+06$ & $1.95 \mathrm{E}+06$ & $4.84 \mathrm{E}-03$ & $4.76 \mathrm{E}-03$ \\
\hline BY-110 & $1.00 \mathrm{E}+04$ & $1.91 \mathrm{E}+06$ & $5.01 \mathrm{E}+02$ & $3.56 \mathrm{E}+03$ & $3.18 \mathrm{E}+04$ & $1.91 \mathrm{E}+06$ & $1.59 \mathrm{E}+03$ & $1.15 \mathrm{E}+04$ & $1.92 \mathrm{E}+06$ & $1.95 \mathrm{E}+06$ & $4.84 \mathrm{E}-03$ & $4.76 \mathrm{E}-03$ \\
\hline BY-111 & $1.00 \mathrm{E}+04$ & $1.91 \mathrm{E}+06$ & $5.01 \mathrm{E}+02$ & $3.56 \mathrm{E}+03$ & $3.18 \mathrm{E}+04$ & $1.91 \mathrm{E}+06$ & $1.59 \mathrm{E}+03$ & $1.15 \mathrm{E}+04$ & $1.92 \mathrm{E}+06$ & $1.95 \mathrm{E}+06$ & $4.84 \mathrm{E}-03$ & $4.76 \mathrm{E}-03$ \\
\hline BY-112 & $1.00 \mathrm{E}+04$ & $1.91 \mathrm{E}+06$ & $5.01 \mathrm{E}+02$ & $3.56 \mathrm{E}+03$ & $3.18 \mathrm{E}+04$ & $1.91 \mathrm{E}+06$ & $1.59 \mathrm{E}+03$ & $1.15 \mathrm{E}+04$ & $1.92 \mathrm{E}+06$ & $1.95 \mathrm{E}+06$ & $4.84 \mathrm{E}-03$ & $4.76 \mathrm{E}-03$ \\
\hline $\mathrm{C}-101$ & $1.00 \mathrm{E}+04$ & $1.91 \mathrm{E}+06$ & $5.01 \mathrm{E}+02$ & $3.37 \mathrm{E}+03$ & $3.18 \mathrm{E}+04$ & $1.91 \mathrm{E}+06$ & $1.59 \mathrm{E}+03$ & $1.09 \mathrm{E}+04$ & $1.92 \mathrm{E}+06$ & $1.95 \mathrm{E}+06$ & $4.89 \mathrm{E}-03$ & $4.82 \mathrm{E}-03$ \\
\hline C-102 & $1.00 \mathrm{E}+04$ & $1.91 \mathrm{E}+06$ & $5.01 \mathrm{E}+02$ & $3.37 \mathrm{E}+03$ & $3.18 \mathrm{E}+04$ & $1.91 \mathrm{E}+06$ & $1.59 \mathrm{E}+03$ & $1.09 \mathrm{E}+04$ & $1.92 \mathrm{E}+06$ & $1.95 \mathrm{E}+06$ & $4.89 \mathrm{E}-03$ & $4.82 \mathrm{E}-03$ \\
\hline C-103 & $1.00 \mathrm{E}+04$ & $1.91 \mathrm{E}+06$ & $5.01 \mathrm{E}+02$ & $3.37 \mathrm{E}+03$ & $3.18 \mathrm{E}+04$ & $1.91 \mathrm{E}+06$ & $1.59 \mathrm{E}+03$ & $1.09 \mathrm{E}+04$ & $1.92 \mathrm{E}+06$ & $1.95 \mathrm{E}+06$ & $4.89 \mathrm{E}-03$ & $4.82 \mathrm{E}-03$ \\
\hline C- 104 & $1.00 \mathrm{E}+04$ & $1.91 \mathrm{E}+06$ & $5.01 E+02$ & $3.37 \mathrm{E}+03$ & $3.18 \mathrm{E}+04$ & $1.91 E+06$ & $1.59 \mathrm{E}+03$ & $1.09 \mathrm{E}+04$ & $1.92 \mathrm{E}+06$ & $1.95 \mathrm{E}+06$ & $4.89 \mathrm{E}-03$ & $4.82 \mathrm{E}-03$ \\
\hline C-105 & $1.00 \mathrm{E}+04$ & $1.91 \mathrm{E}+06$ & $5.01 \mathrm{E}+02$ & $3.37 \mathrm{E}+03$ & $3.18 \mathrm{E}+04$ & $1.91 \mathrm{E}+06$ & $1.59 \mathrm{E}+03$ & $1.09 \mathrm{E}+04$ & $1.92 \mathrm{E}+06$ & $1.95 \mathrm{E}+06$ & $4.89 \mathrm{E}-03$ & $4.82 \mathrm{E}-03$ \\
\hline C-106 & $1.00 \mathrm{E}+04$ & $1.91 \mathrm{E}+06$ & $5.01 \mathrm{E}+02$ & $3.37 \mathrm{E}+03$ & $3.18 \mathrm{E}+04$ & $1.91 \mathrm{E}+06$ & $1.59 \mathrm{E}+03$ & $1.09 \mathrm{E}+04$ & $1.92 \mathrm{E}+06$ & $1.95 \mathrm{E}+06$ & $4.89 \mathrm{E}-03$ & $4.82 \mathrm{E}-03$ \\
\hline$C-107$ & $1.00 \mathrm{E}+04$ & $1.91 \mathrm{E}+06$ & $5.01 \mathrm{E}+02$ & $3.37 \mathrm{E}+03$ & $3.18 \mathrm{E}+04$ & $1.91 \mathrm{E}+06$ & $1.59 \mathrm{E}+03$ & $1.09 \mathrm{E}+04$ & $1.92 \mathrm{E}+06$ & $1.95 \mathrm{E}+06$ & $4.89 \mathrm{E}-03$ & $4.82 \mathrm{E}-03$ \\
\hline C-108 & $1.00 \mathrm{E}+04$ & $1.91 \mathrm{E}+06$ & $5.01 E+02$ & $3.37 \mathrm{E}+03$ & $3.18 \mathrm{E}+04$ & $1.91 E+06$ & $1.59 \mathrm{E}+03$ & $1.09 \mathrm{E}+04$ & $1.92 \mathrm{E}+06$ & $1.95 \mathrm{E}+06$ & $4.89 \mathrm{E}-03$ & $4.82 \mathrm{E}-03$ \\
\hline C-109 & $1.00 \mathrm{E}+04$ & $1.91 \mathrm{E}+06$ & $5.01 \mathrm{E}+02$ & $3.37 \mathrm{E}+03$ & $3.18 \mathrm{E}+04$ & $1.91 \mathrm{E}+06$ & $1.59 \mathrm{E}+03$ & $1.09 \mathrm{E}+04$ & $1.92 \mathrm{E}+06$ & $1.95 \mathrm{E}+06$ & $4.89 \mathrm{E}-03$ & $4.82 \mathrm{E}-03$ \\
\hline C-110 & $1.00 \mathrm{E}+04$ & $1.91 \mathrm{E}+06$ & $5.01 \mathrm{E}+02$ & $3.37 \mathrm{E}+03$ & $3.18 \mathrm{E}+04$ & $1.91 \mathrm{E}+06$ & $1.59 \mathrm{E}+03$ & $1.09 \mathrm{E}+04$ & $1.92 \mathrm{E}+06$ & $1.95 E+06$ & $4.89 \mathrm{E}-03$ & $4.82 \mathrm{E}-03$ \\
\hline $\mathrm{C}-111$ & $1.00 \mathrm{E}+04$ & $1.91 \mathrm{E}+06$ & $5.01 \mathrm{E}+02$ & $3.37 \mathrm{E}+03$ & $3.18 \mathrm{E}+04$ & $1.91 \mathrm{E}+06$ & $1.59 \mathrm{E}+03$ & $1.09 \mathrm{E}+04$ & $1.92 \mathrm{E}+06$ & $1.95 \mathrm{E}+06$ & $4.89 \mathrm{E}-03$ & $4.82 \mathrm{E}-03$ \\
\hline C-112 & $1.00 \mathrm{E}+04$ & $1.91 \mathrm{E}+06$ & $5.01 \mathrm{E}+02$ & $3.37 \mathrm{E}+03$ & $3.18 \mathrm{E}+04$ & $1.91 \mathrm{E}+06$ & $1.59 \mathrm{E}+03$ & $1.09 \mathrm{E}+04$ & $1.92 \mathrm{E}+06$ & $1.95 \mathrm{E}+06$ & $4.89 \mathrm{E}-03$ & $4.82 \mathrm{E}-03$ \\
\hline $\mathrm{C}-201$ & $8.02 \mathrm{E}+03$ & $1.91 \mathrm{E}+06$ & $0.00 \mathrm{E}+00$ & $2.79 \mathrm{E}+03$ & $2.54 \mathrm{E}+04$ & $1.91 \mathrm{E}+06$ & $0.00 \mathrm{E}+00$ & $9.05 \mathrm{E}+03$ & $1.92 \mathrm{E}+06$ & $1.94 \mathrm{E}+06$ & $1.61 \mathrm{E}-04$ & $1.60 \mathrm{E}-04$ \\
\hline C-202 & $8.02 \mathrm{E}+03$ & $1.91 \mathrm{E}+06$ & $0.00 \mathrm{E}+00$ & $2.79 \mathrm{E}+03$ & $2.54 \mathrm{E}+04$ & $1.91 \mathrm{E}+06$ & $0.00 \mathrm{E}+00$ & $9.05 \mathrm{E}+03$ & $1.92 \mathrm{E}+06$ & $1.94 \mathrm{E}+06$ & $1.61 \mathrm{E}-04$ & $1.60 \mathrm{E}-04$ \\
\hline C-203 & $8.02 \mathrm{E}+03$ & $1.91 \mathrm{E}+06$ & $0.00 \mathrm{E}+00$ & $2.79 \mathrm{E}+03$ & $2.54 \mathrm{E}+04$ & $1.91 \mathrm{E}+06$ & $0.00 \mathrm{E}+00$ & $9.05 \mathrm{E}+03$ & $1.92 \mathrm{E}+06$ & $1.94 \mathrm{E}+06$ & $1.61 \mathrm{E}-04$ & $1.60 \mathrm{E}-04$ \\
\hline C-204 & $8.02 \mathrm{E}+03$ & $1.91 \mathrm{E}+06$ & $0.00 \mathrm{E}+00$ & $2.79 \mathrm{E}+03$ & $2.54 \mathrm{E}+04$ & $1.91 \mathrm{E}+06$ & $0.00 \mathrm{E}+00$ & $9.05 \mathrm{E}+03$ & $1.92 \mathrm{E}+06$ & $1.94 \mathrm{E}+06$ & $1.61 \mathrm{E}-04$ & $1.60 \mathrm{E}-04$ \\
\hline S-101 & $1.00 \mathrm{E}+04$ & $1.91 \mathrm{E}+06$ & $5.01 E+02$ & $3.56 \mathrm{E}+03$ & $3.18 \mathrm{E}+04$ & $1.91 \mathrm{E}+06$ & $1.59 \mathrm{E}+03$ & $1.15 \mathrm{E}+04$ & $1.92 \mathrm{E}+06$ & $1.95 \mathrm{E}+06$ & $4.84 \mathrm{E}-03$ & $4.76 \mathrm{E}-03$ \\
\hline S-102 & $1.00 \mathrm{E}+04$ & $1.91 \mathrm{E}+06$ & $5.01 \mathrm{E}+02$ & $3.56 \mathrm{E}+03$ & $3.18 \mathrm{E}+04$ & $1.91 \mathrm{E}+06$ & $1.59 \mathrm{E}+03$ & $1.15 \mathrm{E}+04$ & $1.92 \mathrm{E}+06$ & $1.95 E+06$ & $4.84 \mathrm{E}-03$ & $4.76 \mathrm{E}-03$ \\
\hline S-103 & $1.00 \mathrm{E}+04$ & $1.91 E+06$ & $5.01 \mathrm{E}+02$ & $3.56 \mathrm{E}+03$ & $3.18 \mathrm{E}+04$ & $1.91 \mathrm{E}+06$ & $1.59 \mathrm{E}+03$ & $1.15 \mathrm{E}+04$ & $1.92 \mathrm{E}+06$ & $1.95 \mathrm{E}+06$ & $4.84 \mathrm{E}-03$ & $4.76 \mathrm{E}-03$ \\
\hline S-104 & $1.00 \mathrm{E}+04$ & $1.91 \mathrm{E}+06$ & $5.01 \mathrm{E}+02$ & $3.56 \mathrm{E}+03$ & $3.18 \mathrm{E}+04$ & $1.91 \mathrm{E}+06$ & $1.59 \mathrm{E}+03$ & $1.15 \mathrm{E}+04$ & $1.92 \mathrm{E}+06$ & $1.95 \mathrm{E}+06$ & $4.84 \mathrm{E}-03$ & $4.76 \mathrm{E}-03$ \\
\hline S-105 & $1.00 \mathrm{E}+04$ & $1.91 \mathrm{E}+06$ & $5.01 \mathrm{E}+02$ & $3.56 \mathrm{E}+03$ & $3.18 \mathrm{E}+04$ & $1.91 \mathrm{E}+06$ & $1.59 \mathrm{E}+03$ & $1.15 \mathrm{E}+04$ & $1.92 \mathrm{E}+06$ & $1.95 \mathrm{E}+06$ & $4.84 \mathrm{E}-03$ & $4.76 \mathrm{E}-03$ \\
\hline S-106 & $1.00 \mathrm{E}+04$ & $1.91 \mathrm{E}+06$ & $5.01 \mathrm{E}+02$ & $3.56 \mathrm{E}+03$ & $3.18 \mathrm{E}+04$ & $1.91 \mathrm{E}+06$ & $1.59 \mathrm{E}+03$ & $1.15 \mathrm{E}+04$ & $1.92 \mathrm{E}+06$ & $1.95 \mathrm{E}+06$ & $4.84 \mathrm{E}-03$ & $4.76 \mathrm{E}-03$ \\
\hline S-107 & $1.00 \mathrm{E}+04$ & $1.91 \mathrm{E}+06$ & $5.01 \mathrm{E}+02$ & $3.56 \mathrm{E}+03$ & $3.18 \mathrm{E}+04$ & $1.91 \mathrm{E}+06$ & $1.59 \mathrm{E}+03$ & $1.15 \mathrm{E}+04$ & $1.92 \mathrm{E}+06$ & $1.95 \mathrm{E}+06$ & $4.84 \mathrm{E}-03$ & $4.76 \mathrm{E}-03$ \\
\hline S-108 & $1.00 \mathrm{E}+04$ & $1.91 \mathrm{E}+06$ & $5.01 \mathrm{E}+02$ & $3.56 \mathrm{E}+03$ & $3.18 \mathrm{E}+04$ & $1.91 \mathrm{E}+06$ & $1.59 \mathrm{E}+03$ & $1.15 \mathrm{E}+04$ & $1.92 \mathrm{E}+06$ & $1.95 \mathrm{E}+06$ & $4.84 \mathrm{E}-03$ & $4.76 \mathrm{E}-03$ \\
\hline S-109 & $1.00 \mathrm{E}+04$ & $1.91 \mathrm{E}+06$ & $5.01 \mathrm{E}+02$ & $3.56 \mathrm{E}+03$ & $3.18 \mathrm{E}+04$ & $1.91 \mathrm{E}+06$ & $1.59 \mathrm{E}+03$ & $1.15 \mathrm{E}+04$ & $1.92 \mathrm{E}+06$ & $1.95 \mathrm{E}+06$ & $4.84 \mathrm{E}-03$ & $4.76 \mathrm{E}-03$ \\
\hline S-110 & $1.00 \mathrm{E}+04$ & $1.91 \mathrm{E}+06$ & $5.01 \mathrm{E}+02$ & $3.56 \mathrm{E}+03$ & $3.18 \mathrm{E}+04$ & $1.91 \mathrm{E}+06$ & $1.59 \mathrm{E}+03$ & $1.15 \mathrm{E}+04$ & $1.92 \mathrm{E}+06$ & $1.95 \mathrm{E}+06$ & $4.84 \mathrm{E}-03$ & $4.76 \mathrm{E}-03$ \\
\hline $\mathrm{S}-111$ & $1.00 \mathrm{E}+04$ & $1.91 \mathrm{E}+06$ & $5.01 E+02$ & $3.56 \mathrm{E}+03$ & $3.18 \mathrm{E}+04$ & $1.91 \mathrm{E}+06$ & $1.59 \mathrm{E}+03$ & $1.15 \mathrm{E}+04$ & $1.92 \mathrm{E}+06$ & $1.95 \mathrm{E}+06$ & $4.84 \mathrm{E}-03$ & $4.76 \mathrm{E}-03$ \\
\hline $\mathrm{S}-112$ & $1.00 \mathrm{E}+04$ & $1.91 \mathrm{E}+06$ & $5.01 \mathrm{E}+02$ & $3.56 \mathrm{E}+03$ & $3.18 \mathrm{E}+04$ & $1.91 \mathrm{E}+06$ & $1.59 \mathrm{E}+03$ & $1.15 \mathrm{E}+04$ & $1.92 \mathrm{E}+06$ & $1.95 \mathrm{E}+06$ & $4.84 \mathrm{E}-03$ & $4.76 \mathrm{E}-03$ \\
\hline SX-101 & $1.00 \mathrm{E}+04$ & $0.00 \mathrm{E}+00$ & $0.00 \mathrm{E}+00$ & $3.05 \mathrm{E}+03$ & $3.18 \mathrm{E}+04$ & $0.00 \mathrm{E}+00$ & $0.00 \mathrm{E}+00$ & $9.89 \mathrm{E}+03$ & $1.31 \mathrm{E}+04$ & $4.16 \mathrm{E}+04$ & $7.11 \mathrm{E}-01$ & $2.23 \mathrm{E}-01$ \\
\hline SX-102 & $1.00 \mathrm{E}+04$ & $0.00 \mathrm{E}+00$ & $0.00 \mathrm{E}+00$ & $3.05 \mathrm{E}+03$ & $3.18 \mathrm{E}+04$ & $0.00 \mathrm{E}+00$ & $0.00 \mathrm{E}+00$ & $9.89 \mathrm{E}+03$ & $1.31 E+04$ & $4.16 \mathrm{E}+04$ & $7.11 \mathrm{E}-01$ & $2.23 \mathrm{E}-01$ \\
\hline SX-103 & $1.00 \mathrm{E}+04$ & $0.00 \mathrm{E}+00$ & $0.00 \mathrm{E}+00$ & $3.05 \mathrm{E}+03$ & $3.18 \mathrm{E}+04$ & $0.00 \mathrm{E}+00$ & $0.00 \mathrm{E}+00$ & $9.89 \mathrm{E}+03$ & $1.31 \mathrm{E}+04$ & $4.16 \mathrm{E}+04$ & $7.11 \mathrm{E}-01$ & $2.23 \mathrm{E}-01$ \\
\hline SX-104 & $1.00 \mathrm{E}+04$ & $0.00 \mathrm{E}+00$ & $0.00 \mathrm{E}+00$ & $3.05 \mathrm{E}+03$ & $3.18 \mathrm{E}+04$ & $0.00 \mathrm{E}+00$ & $0.00 \mathrm{E}+00$ & $9.89 \mathrm{E}+03$ & $1.31 \mathrm{E}+04$ & $4.16 \mathrm{E}+04$ & $7.11 \mathrm{E}-01$ & $2.23 \mathrm{E}-01$ \\
\hline SX-105 & $1.00 \mathrm{E}+04$ & $0.00 \mathrm{E}+00$ & $0.00 \mathrm{E}+00$ & $3.05 E+03$ & $3.18 \mathrm{E}+04$ & $0.00 \mathrm{E}+00$ & $0.00 \mathrm{E}+00$ & $9.89 \mathrm{E}+03$ & $1.31 \mathrm{E}+04$ & $4.16 \mathrm{E}+04$ & $7.11 \mathrm{E}-01$ & $2.23 \mathrm{E}-01$ \\
\hline SX-106 & $1.00 \mathrm{E}+04$ & $0.00 \mathrm{E}+00$ & $0.00 \mathrm{E}+00$ & $3.05 \mathrm{E}+03$ & $3.18 \mathrm{E}+04$ & $0.00 \mathrm{E}+00$ & $0.00 \mathrm{E}+00$ & $9.89 \mathrm{E}+03$ & $1.31 \mathrm{E}+04$ & $4.16 \mathrm{E}+04$ & $7.11 \mathrm{E}-01$ & $2.23 \mathrm{E}-01$ \\
\hline
\end{tabular}


Table B-2. The Diffusion Resistance and Gas Diffusion Rates of Hydrogen and Methane for 149 Single-Shell Tanks.

\begin{tabular}{|c|c|c|c|c|c|c|c|c|c|c|c|c|}
\hline Tank & $\begin{array}{c}\text { Concrete } \\
\text { Resist of } \mathbf{H}_{2} \\
(\mathrm{sec} / \mathrm{cm})\end{array}$ & $\begin{array}{c}\text { Asphaltic } \\
\text { Resist of } \mathbf{H}_{2} \\
\text { (sec/cm) }\end{array}$ & $\begin{array}{c}\text { Gunite } \\
\text { Resist of } \mathrm{H}_{2} \\
(\mathrm{sec} / \mathrm{cm})\end{array}$ & $\begin{array}{c}\text { Soil } \\
\text { Resist of } \mathbf{H}_{2} \\
(\mathrm{sec} / \mathrm{cm})\end{array}$ & $\begin{array}{c}\text { Concrete } \\
\text { Resist of CH} \\
(\mathrm{sec} / \mathrm{cm})\end{array}$ & $\begin{array}{c}\text { Asphaltic } \\
\text { Resist of CH } \\
\text { (sec/cm) }\end{array}$ & $\begin{array}{c}\begin{array}{c}\text { Gunite } \\
\text { Resist of } \mathrm{CH}_{4} \\
\text { (sec/cm) }\end{array} \\
\end{array}$ & $\begin{array}{c}\begin{array}{c}\text { Soil } \\
\text { Resist of CH } \\
\text { (sec/cm) }\end{array} \\
\end{array}$ & $\begin{array}{c}\text { Total } \\
\text { Resist of } \mathbf{H}_{2} \\
\text { (sec/cm) }\end{array}$ & \begin{tabular}{|c|}
$\begin{array}{c}\text { Total } \\
\text { Resist of CH} \\
\text { (sec/cm) }\end{array}$ \\
\end{tabular} & $\begin{array}{c}\mathbf{H}_{2} \text { Diff Rate } \\
\mathbf{A i} / \mathbf{R i} \\
(\mathrm{cfm}) \\
\end{array}$ & $\begin{array}{c}\mathrm{CH}_{\mathbf{4}} \text { Diff Rate } \\
\mathbf{A i} / \mathbf{R i} \\
(\mathrm{cfm})\end{array}$ \\
\hline SX-107 & $1.00 \mathrm{E}+04$ & $0.00 \mathrm{E}+00$ & $0.00 \mathrm{E}+00$ & $3.05 \mathrm{E}+03$ & $3.18 \mathrm{E}+04$ & $0.00 \mathrm{E}+00$ & $0.00 \mathrm{E}+00$ & $9.89 \mathrm{E}+03$ & $1.31 \mathrm{E}+04$ & $4.16 \mathrm{E}+04$ & $7.11 \mathrm{E}-01$ & $2.23 \mathrm{E}-01$ \\
\hline SX-108 & $1.00 \mathrm{E}+04$ & $0.00 \mathrm{E}+00$ & $0.00 \mathrm{E}+00$ & $3.05 E+03$ & $3.18 \mathrm{E}+04$ & $0.00 \mathrm{E}+00$ & $0.00 \mathrm{E}+00$ & $9.89 \mathrm{E}+03$ & $1.31 \mathrm{E}+04$ & $4.16 \mathrm{E}+04$ & $7.11 \mathrm{E}-01$ & $2.23 \mathrm{E}-01$ \\
\hline SX-109 & $1.00 \mathrm{E}+04$ & $0.00 \mathrm{E}+00$ & $0.00 \mathrm{E}+00$ & $3.05 \mathrm{E}+03$ & $3.18 \mathrm{E}+04$ & $0.00 \mathrm{E}+00$ & $0.00 \mathrm{E}+00$ & $9.89 \mathrm{E}+03$ & $1.31 \mathrm{E}+04$ & $4.16 \mathrm{E}+04$ & $7.11 \mathrm{E}-01$ & $2.23 \mathrm{E}-01$ \\
\hline SX-110 & $1.00 \mathrm{E}+04$ & $0.00 \mathrm{E}+00$ & $0.00 \mathrm{E}+00$ & $3.05 \mathrm{E}+03$ & $3.18 \mathrm{E}+04$ & $0.00 \mathrm{E}+00$ & $0.00 \mathrm{E}+00$ & $9.89 \mathrm{E}+03$ & $1.31 E+04$ & $4.16 \mathrm{E}+04$ & $7.11 \mathrm{E}-01$ & $2.23 \mathrm{E}-01$ \\
\hline SX-111 & $1.00 \mathrm{E}+04$ & $0.00 \mathrm{E}+00$ & $0.00 \mathrm{E}+00$ & $3.05 \mathrm{E}+03$ & $3.18 \mathrm{E}+04$ & $0.00 \mathrm{E}+00$ & $0.00 \mathrm{E}+00$ & $9.89 \mathrm{E}+03$ & $1.31 \mathrm{E}+04$ & $4.16 \mathrm{E}+04$ & $7.11 \mathrm{E}-01$ & $2.23 \mathrm{E}-01$ \\
\hline SX-112 & $1.00 \mathrm{E}+04$ & $0.00 \mathrm{E}+00$ & $0.00 \mathrm{E}+00$ & $3.05 \mathrm{E}+03$ & $3.18 \mathrm{E}+04$ & $0.00 \mathrm{E}+00$ & $0.00 \mathrm{E}+00$ & $9.89 \mathrm{E}+03$ & $1.31 \mathrm{E}+04$ & $4.16 \mathrm{E}+04$ & $7.11 \mathrm{E}-01$ & $2.23 \mathrm{E}-01$ \\
\hline SX-113 & $1.00 \mathrm{E}+04$ & $0.00 \mathrm{E}+00$ & $0.00 \mathrm{E}+00$ & $3.05 \mathrm{E}+03$ & $3.18 \mathrm{E}+04$ & $0.00 \mathrm{E}+00$ & $0.00 \mathrm{E}+00$ & $9.89 \mathrm{E}+03$ & $1.31 \mathrm{E}+04$ & $4.16 \mathrm{E}+04$ & $7.11 \mathrm{E}-01$ & $2.23 \mathrm{E}-01$ \\
\hline SX-114 & $1.00 \mathrm{E}+04$ & $0.00 \mathrm{E}+00$ & $0.00 \mathrm{E}+00$ & $3.05 \mathrm{E}+03$ & $3.18 \mathrm{E}+04$ & $0.00 \mathrm{E}+00$ & $0.00 \mathrm{E}+00$ & $9.89 \mathrm{E}+03$ & $1.31 \mathrm{E}+04$ & $4.16 \mathrm{E}+04$ & $7.11 \mathrm{E}-01$ & $2.23 \mathrm{E}-01$ \\
\hline SX-115 & $1.00 \mathrm{E}+04$ & $0.00 \mathrm{E}+00$ & $0.00 \mathrm{E}+00$ & $3.05 \mathrm{E}+03$ & $3.18 \mathrm{E}+04$ & $0.00 \mathrm{E}+00$ & $0.00 \mathrm{E}+00$ & $9.89 \mathrm{E}+03$ & $1.31 \mathrm{E}+04$ & $4.16 \mathrm{E}+04$ & $7.11 \mathrm{E}-01$ & $2.23 \mathrm{E}-01$ \\
\hline $\mathrm{T}-101$ & $1.00 \mathrm{E}+04$ & $1.91 \mathrm{E}+06$ & $5.01 \mathrm{E}+02$ & $3.37 \mathrm{E}+03$ & $3.18 \mathrm{E}+04$ & $1.91 \mathrm{E}+06$ & $1.59 \mathrm{E}+03$ & $1.09 \mathrm{E}+04$ & $1.92 \mathrm{E}+06$ & $1.95 \mathrm{E}+06$ & $4.89 \mathrm{E}-03$ & $4.82 \mathrm{E}-03$ \\
\hline$T-102$ & $1.00 \mathrm{E}+04$ & $1.91 \mathrm{E}+06$ & $5.01 \mathrm{E}+02$ & $3.37 \mathrm{E}+03$ & $3.18 \mathrm{E}+04$ & $1.91 \mathrm{E}+06$ & $1.59 \mathrm{E}+03$ & $1.09 \mathrm{E}+04$ & $1.92 \mathrm{E}+06$ & $1.95 \mathrm{E}+06$ & $4.89 \mathrm{E}-03$ & 4.82E-03 \\
\hline$T-103$ & $1.00 \mathrm{E}+04$ & $1.91 \mathrm{E}+06$ & $5.01 \mathrm{E}+02$ & $3.37 \mathrm{E}+03$ & $3.18 \mathrm{E}+04$ & $1.91 \mathrm{E}+06$ & $1.59 \mathrm{E}+03$ & $1.09 \mathrm{E}+04$ & $1.92 \mathrm{E}+06$ & $1.95 \mathrm{E}+06$ & $4.89 \mathrm{E}-03$ & $4.82 \mathrm{E}-03$ \\
\hline $\mathrm{T}-104$ & $1.00 \mathrm{E}+04$ & $1.91 \mathrm{E}+06$ & $5.01 \mathrm{E}+02$ & $3.37 \mathrm{E}+03$ & $3.18 \mathrm{E}+04$ & $1.91 \mathrm{E}+06$ & $1.59 \mathrm{E}+03$ & $1.09 \mathrm{E}+04$ & $1.92 \mathrm{E}+06$ & $1.95 \mathrm{E}+06$ & $4.89 \mathrm{E}-03$ & $4.82 \mathrm{E}-03$ \\
\hline $\mathrm{T}-105$ & $1.00 \mathrm{E}+04$ & $1.91 \mathrm{E}+06$ & $5.01 \mathrm{E}+02$ & $3.37 \mathrm{E}+03$ & $3.18 \mathrm{E}+04$ & $1.91 \mathrm{E}+06$ & $1.59 \mathrm{E}+03$ & $1.09 \mathrm{E}+04$ & $1.92 \mathrm{E}+06$ & $1.95 E+06$ & $4.89 \mathrm{E}-03$ & $4.82 \mathrm{E}-03$ \\
\hline T-106 & $1.00 \mathrm{E}+04$ & $1.91 \mathrm{E}+06$ & $5.01 \mathrm{E}+02$ & $3.37 \mathrm{E}+03$ & $3.18 \mathrm{E}+04$ & $1.91 \mathrm{E}+06$ & $1.59 \mathrm{E}+03$ & $1.09 \mathrm{E}+04$ & $1.92 \mathrm{E}+06$ & $1.95 \mathrm{E}+06$ & $4.89 \mathrm{E}-03$ & $4.82 \mathrm{E}-03$ \\
\hline$T-107$ & $1.00 \mathrm{E}+04$ & $1.91 \mathrm{E}+06$ & $5.01 \mathrm{E}+02$ & $3.37 \mathrm{E}+03$ & $3.18 \mathrm{E}+04$ & $1.91 \mathrm{E}+06$ & $1.59 \mathrm{E}+03$ & $1.09 \mathrm{E}+04$ & $1.92 \mathrm{E}+06$ & $1.95 \mathrm{E}+06$ & $4.89 \mathrm{E}-03$ & 4.82E-03 \\
\hline T-108 & $1.00 \mathrm{E}+04$ & $1.91 \mathrm{E}+06$ & $5.01 \mathrm{E}+02$ & $3.37 \mathrm{E}+03$ & $3.18 \mathrm{E}+04$ & $1.91 \mathrm{E}+06$ & $1.59 \mathrm{E}+03$ & $1.09 \mathrm{E}+04$ & $1.92 \mathrm{E}+06$ & $1.95 \mathrm{E}+06$ & $4.89 \mathrm{E}-03$ & $4.82 \mathrm{E}-03$ \\
\hline $\mathrm{T}-109$ & $1.00 \mathrm{E}+04$ & $1.91 \mathrm{E}+06$ & $5.01 \mathrm{E}+02$ & $3.37 \mathrm{E}+03$ & $3.18 \mathrm{E}+04$ & $1.91 \mathrm{E}+06$ & $1.59 \mathrm{E}+03$ & $1.09 \mathrm{E}+04$ & $1.92 \mathrm{E}+06$ & $1.95 \mathrm{E}+06$ & $4.89 \mathrm{E}-03$ & $4.82 \mathrm{E}-03$ \\
\hline $\mathrm{T}-110$ & $1.00 \mathrm{E}+04$ & $1.91 \mathrm{E}+06$ & $5.01 \mathrm{E}+02$ & $3.37 \mathrm{E}+03$ & $3.18 \mathrm{E}+04$ & $1.91 \mathrm{E}+06$ & $1.59 \mathrm{E}+03$ & $1.09 \mathrm{E}+04$ & $1.92 \mathrm{E}+06$ & $1.95 \mathrm{E}+06$ & $4.89 \mathrm{E}-03$ & $4.82 \mathrm{E}-03$ \\
\hline $\mathrm{T}-111$ & $1.00 \mathrm{E}+04$ & $1.91 \mathrm{E}+06$ & $5.01 \mathrm{E}+02$ & $3.37 \mathrm{E}+03$ & $3.18 \mathrm{E}+04$ & $1.91 \mathrm{E}+06$ & $1.59 \mathrm{E}+03$ & $1.09 \mathrm{E}+04$ & $1.92 \mathrm{E}+06$ & $1.95 \mathrm{E}+06$ & $4.89 \mathrm{E}-03$ & $4.82 \mathrm{E}-03$ \\
\hline$T-112$ & $1.00 \mathrm{E}+04$ & $1.91 \mathrm{E}+06$ & $5.01 E+02$ & $3.37 \mathrm{E}+03$ & $3.18 \mathrm{E}+04$ & $1.91 \mathrm{E}+06$ & $1.59 \mathrm{E}+03$ & $1.09 \mathrm{E}+04$ & $1.92 \mathrm{E}+06$ & $1.95 \mathrm{E}+06$ & $4.89 \mathrm{E}-03$ & $4.82 \mathrm{E}-03$ \\
\hline T-201 & $8.02 \mathrm{E}+03$ & $1.91 \mathrm{E}+06$ & $0.00 \mathrm{E}+00$ & $2.79 \mathrm{E}+03$ & $2.54 \mathrm{E}+04$ & $1.91 \mathrm{E}+06$ & $0.00 \mathrm{E}+00$ & $9.05 \mathrm{E}+03$ & $1.92 \mathrm{E}+06$ & $1.94 \mathrm{E}+06$ & $1.61 \mathrm{E}-04$ & $1.60 \mathrm{E}-04$ \\
\hline T-202 & $8.02 \mathrm{E}+03$ & $1.91 \mathrm{E}+06$ & $0.00 \mathrm{E}+00$ & $2.79 \mathrm{E}+03$ & $2.54 \mathrm{E}+04$ & $1.91 \mathrm{E}+06$ & $0.00 \mathrm{E}+00$ & $9.05 \mathrm{E}+03$ & $1.92 \mathrm{E}+06$ & $1.94 \mathrm{E}+06$ & $1.61 \mathrm{E}-04$ & $1.60 \mathrm{E}-04$ \\
\hline $\mathrm{T}-203$ & $8.02 \mathrm{E}+03$ & $1.91 \mathrm{E}+06$ & $0.00 \mathrm{E}+00$ & $2.79 \mathrm{E}+03$ & $2.54 \mathrm{E}+04$ & $1.91 \mathrm{E}+06$ & $0.00 \mathrm{E}+00$ & $9.05 \mathrm{E}+03$ & $1.92 \mathrm{E}+06$ & $1.94 \mathrm{E}+06$ & $1.61 \mathrm{E}-04$ & $1.60 \mathrm{E}-04$ \\
\hline $\mathrm{T}-204$ & $8.02 \mathrm{E}+03$ & $1.91 \mathrm{E}+06$ & $0.00 \mathrm{E}+00$ & $2.79 \mathrm{E}+03$ & $2.54 \mathrm{E}+04$ & $1.91 \mathrm{E}+06$ & $0.00 \mathrm{E}+00$ & $9.05 \mathrm{E}+03$ & $1.92 \mathrm{E}+06$ & $1.94 \mathrm{E}+06$ & $1.61 \mathrm{E}-04$ & $1.60 \mathrm{E}-04$ \\
\hline TX-101 & $1.00 \mathrm{E}+04$ & $1.91 \mathrm{E}+06$ & $5.01 \mathrm{E}+02$ & $3.56 \mathrm{E}+03$ & $3.18 \mathrm{E}+04$ & $1.91 \mathrm{E}+06$ & $1.59 \mathrm{E}+03$ & $1.15 \mathrm{E}+04$ & $1.92 \mathrm{E}+06$ & $1.95 \mathrm{E}+06$ & 4.84E-03 & $4.76 \mathrm{E}-03$ \\
\hline TX-102 & $1.00 \mathrm{E}+04$ & $1.91 E+06$ & $5.01 \mathrm{E}+02$ & $3.56 \mathrm{E}+03$ & $3.18 \mathrm{E}+04$ & $1.91 \mathrm{E}+06$ & $1.59 \mathrm{E}+03$ & $1.15 \mathrm{E}+04$ & $1.92 \mathrm{E}+06$ & $1.95 \mathrm{E}+06$ & $4.84 \mathrm{E}-03$ & $4.76 \mathrm{E}-03$ \\
\hline TX-103 & $1.00 \mathrm{E}+04$ & $1.91 \mathrm{E}+06$ & $5.01 \mathrm{E}+02$ & $3.56 \mathrm{E}+03$ & $3.18 \mathrm{E}+04$ & $1.91 \mathrm{E}+06$ & $1.59 \mathrm{E}+03$ & $1.15 \mathrm{E}+04$ & $1.92 \mathrm{E}+06$ & $1.95 \mathrm{E}+06$ & $4.84 \mathrm{E}-03$ & $4.76 \mathrm{E}-03$ \\
\hline TX-104 & $1.00 \mathrm{E}+04$ & $1.91 \mathrm{E}+06$ & $5.01 \mathrm{E}+02$ & $3.56 \mathrm{E}+03$ & $3.18 \mathrm{E}+04$ & $1.91 \mathrm{E}+06$ & $1.59 \mathrm{E}+03$ & $1.15 \mathrm{E}+04$ & $1.92 E+06$ & $1.95 \mathrm{E}+06$ & $4.84 \mathrm{E}-03$ & 4.76E-03 \\
\hline TX-105 & $1.00 \mathrm{E}+04$ & $1.91 \mathrm{E}+06$ & $5.01 \mathrm{E}+02$ & $3.56 \mathrm{E}+03$ & $3.18 \mathrm{E}+04$ & $1.91 \mathrm{E}+06$ & $1.59 \mathrm{E}+03$ & $1.15 \mathrm{E}+04$ & $1.92 \mathrm{E}+06$ & $1.95 \mathrm{E}+06$ & $4.84 \mathrm{E}-03$ & $4.76 \mathrm{E}-03$ \\
\hline TX-106 & $1.00 \mathrm{E}+04$ & $1.91 E+06$ & $5.01 \mathrm{E}+02$ & $3.56 \mathrm{E}+03$ & $3.18 E+04$ & $1.91 E+06$ & $1.59 \mathrm{E}+03$ & $1.15 E+04$ & $1.92 \mathrm{E}+06$ & $1.95 \mathrm{E}+06$ & $4.84 \mathrm{E}-03$ & $4.76 \mathrm{E}-03$ \\
\hline TX-107 & $1.00 \mathrm{E}+04$ & $1.91 \mathrm{E}+06$ & $5.01 \mathrm{E}+02$ & $3.56 \mathrm{E}+03$ & $3.18 \mathrm{E}+04$ & $1.91 \mathrm{E}+06$ & $1.59 \mathrm{E}+03$ & $1.15 \mathrm{E}+04$ & $1.92 \mathrm{E}+06$ & $1.95 \mathrm{E}+06$ & $4.84 \mathrm{E}-03$ & $4.76 \mathrm{E}-03$ \\
\hline TX-108 & $1.00 \mathrm{E}+04$ & $1.91 E+06$ & $5.01 \mathrm{E}+02$ & $3.56 \mathrm{E}+03$ & $3.18 \mathrm{E}+04$ & $1.91 \mathrm{E}+06$ & $1.59 \mathrm{E}+03$ & $1.15 \mathrm{E}+04$ & $1.92 \mathrm{E}+06$ & $1.95 \mathrm{E}+06$ & $4.84 \mathrm{E}-03$ & $4.76 \mathrm{E}-03$ \\
\hline TX-109 & $1.00 \mathrm{E}+04$ & $1.91 \mathrm{E}+06$ & $5.01 \mathrm{E}+02$ & $3.56 \mathrm{E}+03$ & $3.18 \mathrm{E}+04$ & $1.91 \mathrm{E}+06$ & $1.59 \mathrm{E}+03$ & $1.15 \mathrm{E}+04$ & $1.92 \mathrm{E}+06$ & $1.95 \mathrm{E}+06$ & $4.84 \mathrm{E}-03$ & $4.76 \mathrm{E}-03$ \\
\hline TX-110 & $1.00 \mathrm{E}+04$ & $1.91 \mathrm{E}+06$ & $5.01 \mathrm{E}+02$ & $3.56 \mathrm{E}+03$ & $3.18 \mathrm{E}+04$ & $1.91 \mathrm{E}+06$ & $1.59 \mathrm{E}+03$ & $1.15 \mathrm{E}+04$ & $1.92 \mathrm{E}+06$ & $1.95 \mathrm{E}+06$ & $4.84 \mathrm{E}-03$ & $4.76 \mathrm{E}-03$ \\
\hline TX-111 & $1.00 \mathrm{E}+04$ & $1.91 \mathrm{E}+06$ & $5.01 E+02$ & $3.56 \mathrm{E}+03$ & $3.18 \mathrm{E}+04$ & $1.91 \mathrm{E}+06$ & $1.59 \mathrm{E}+03$ & $1.15 \mathrm{E}+04$ & $1.92 \mathrm{E}+06$ & $1.95 \mathrm{E}+06$ & $4.84 \mathrm{E}-03$ & 4.76E-03 \\
\hline $\mathrm{TX}-112$ & $1.00 \mathrm{E}+04$ & $1.91 \mathrm{E}+06$ & $5.01 \mathrm{E}+02$ & $3.56 \mathrm{E}+03$ & $3.18 \mathrm{E}+04$ & $1.91 \mathrm{E}+06$ & $1.59 \mathrm{E}+03$ & $1.15 E+04$ & $1.92 \mathrm{E}+06$ & $1.95 \mathrm{E}+06$ & $4.84 \mathrm{E}-03$ & $4.76 \mathrm{E}-03$ \\
\hline TX-113 & $1.00 \mathrm{E}+04$ & $1.91 \mathrm{E}+06$ & $5.01 \mathrm{E}+02$ & $3.56 \mathrm{E}+03$ & $3.18 E+04$ & $1.91 \mathrm{E}+06$ & $1.59 \mathrm{E}+03$ & $1.15 \mathrm{E}+04$ & $1.92 \mathrm{E}+06$ & $1.95 \mathrm{E}+06$ & 4.84E-03 & $4.76 \mathrm{E}-03$ \\
\hline TX-114 & $1.00 \mathrm{E}+04$ & $1.91 E+06$ & $5.01 \mathrm{E}+02$ & $3.56 \mathrm{E}+03$ & $3.18 \mathrm{E}+04$ & $1.91 \mathrm{E}+06$ & $1.59 \mathrm{E}+03$ & $1.15 \mathrm{E}+04$ & $1.92 \mathrm{E}+06$ & $1.95 \mathrm{E}+06$ & $4.84 \mathrm{E}-03$ & $4.76 \mathrm{E}-03$ \\
\hline $\mathrm{TX}-115$ & $1.00 \mathrm{E}+04$ & $1.91 \mathrm{E}+06$ & $5.01 \mathrm{E}+02$ & $3.56 \mathrm{E}+03$ & $3.18 \mathrm{E}+04$ & $1.91 \mathrm{E}+06$ & $1.59 \mathrm{E}+03$ & $1.15 \mathrm{E}+04$ & $1.92 \mathrm{E}+06$ & $1.95 \mathrm{E}+06$ & $4.84 \mathrm{E}-03$ & $4.76 \mathrm{E}-03$ \\
\hline TX-116 & $1.00 \mathrm{E}+04$ & $1.91 E+06$ & $5.01 \mathrm{E}+02$ & $3.56 \mathrm{E}+03$ & $3.18 \mathrm{E}+04$ & $1.91 \mathrm{E}+06$ & $1.59 \mathrm{E}+03$ & $1.15 \mathrm{E}+04$ & $1.92 \mathrm{E}+06$ & $1.95 \mathrm{E}+06$ & 4.84E-03 & $4.76 \mathrm{E}-03$ \\
\hline TX-117 & $1.00 \mathrm{E}+04$ & $1.91 \mathrm{E}+06$ & $5.01 E+02$ & $3.56 \mathrm{E}+03$ & $3.18 \mathrm{E}+04$ & $1.91 \mathrm{E}+06$ & $1.59 \mathrm{E}+03$ & $1.15 \mathrm{E}+04$ & $1.92 \mathrm{E}+06$ & $1.95 \mathrm{E}+06$ & $4.84 \mathrm{E}-03$ & $4.76 \mathrm{E}-03$ \\
\hline TX-118 & $1.00 \mathrm{E}+04$ & $1.91 \mathrm{E}+06$ & $5.01 \mathrm{E}+02$ & $3.56 \mathrm{E}+03$ & $3.18 \mathrm{E}+04$ & $1.91 \mathrm{E}+06$ & $1.59 \mathrm{E}+03$ & $1.15 \mathrm{E}+04$ & $1.92 \mathrm{E}+06$ & $1.95 \mathrm{E}+06$ & 4.84E-03 & $4.76 \mathrm{E}-03$ \\
\hline
\end{tabular}




\section{RPP-5926 REV 9}

Table B-2. The Diffusion Resistance and Gas Diffusion Rates of Hydrogen and Methane for 149 Single-Shell Tanks.

\begin{tabular}{|c|c|c|c|c|c|c|c|c|c|c|c|c|}
\hline Tank & $\begin{array}{c}\text { Concrete } \\
\text { Resist of } \mathbf{H}_{2} \\
(\mathrm{sec} / \mathrm{cm})\end{array}$ & $\begin{array}{c}\text { Asphaltic } \\
\text { Resist of } \mathbf{H}_{2} \\
\text { (sec/cm) }\end{array}$ & $\begin{array}{c}\text { Gunite } \\
\text { Resist of } \mathrm{H}_{2} \\
(\mathrm{sec} / \mathrm{cm})\end{array}$ & $\begin{array}{c}\text { Soil } \\
\text { Resist of } \mathrm{H}_{2} \\
(\mathrm{sec} / \mathrm{cm})\end{array}$ & $\begin{array}{c}\text { Concrete } \\
\text { Resist of CH } \\
(\mathrm{sec} / \mathrm{cm})\end{array}$ & $\begin{array}{c}\text { Asphaltic } \\
\text { Resist of CH } \\
\text { (sec/cm) }\end{array}$ & \begin{tabular}{|c|} 
Gunite \\
Resist of $\mathrm{CH}_{4}$ \\
(sec/cm)
\end{tabular} & $\begin{array}{c}\text { Soil } \\
\text { Resist of CH } \\
\text { (sec/cm) }\end{array}$ & $\begin{array}{c}\text { Total } \\
\text { Resist of } \mathrm{H}_{2} \\
(\mathrm{sec} / \mathrm{cm})\end{array}$ & $\begin{array}{c}\text { Total } \\
\text { Resist of } \mathrm{CH}_{4} \\
(\mathrm{sec} / \mathrm{cm})\end{array}$ & \begin{tabular}{|c|}
$\mathbf{H}_{2}$ Diff Rate \\
$\mathbf{A i} / \mathbf{R i}$ \\
$(\mathrm{cfm})$
\end{tabular} & $\begin{array}{c}\mathrm{CH}_{4} \text { Diff Rate } \\
\mathbf{A i} / \mathbf{R i} \\
(\mathbf{c f m})\end{array}$ \\
\hline TY-101 & $1.00 \mathrm{E}+04$ & $1.91 \mathrm{E}+06$ & $5.01 \mathrm{E}+02$ & $3.56 \mathrm{E}+03$ & $3.18 \mathrm{E}+04$ & $1.91 \mathrm{E}+06$ & $1.59 \mathrm{E}+03$ & $1.15 \mathrm{E}+04$ & $1.92 \mathrm{E}+06$ & $1.95 \mathrm{E}+06$ & $4.84 \mathrm{E}-03$ & $4.76 \mathrm{E}-03$ \\
\hline TY-102 & $1.00 \mathrm{E}+04$ & $1.91 \mathrm{E}+06$ & $5.01 \mathrm{E}+02$ & $3.56 \mathrm{E}+03$ & $3.18 \mathrm{E}+04$ & $1.91 \mathrm{E}+06$ & $1.59 \mathrm{E}+03$ & $1.15 \mathrm{E}+04$ & $1.92 \mathrm{E}+06$ & $1.95 \mathrm{E}+06$ & $4.84 \mathrm{E}-03$ & $4.76 \mathrm{E}-03$ \\
\hline TY-103 & $1.00 \mathrm{E}+04$ & $1.91 \mathrm{E}+06$ & $5.01 \mathrm{E}+02$ & $3.56 \mathrm{E}+03$ & $3.18 \mathrm{E}+04$ & $1.91 \mathrm{E}+06$ & $1.59 \mathrm{E}+03$ & $1.15 \mathrm{E}+04$ & $1.92 E+06$ & $1.95 \mathrm{E}+06$ & $4.84 \mathrm{E}-03$ & $4.76 \mathrm{E}-03$ \\
\hline TY-104 & $1.00 \mathrm{E}+04$ & $1.91 \mathrm{E}+06$ & $5.01 \mathrm{E}+02$ & $3.56 \mathrm{E}+03$ & $3.18 \mathrm{E}+04$ & $1.91 \mathrm{E}+06$ & $1.59 \mathrm{E}+03$ & $1.15 \mathrm{E}+04$ & $1.92 \mathrm{E}+06$ & $1.95 \mathrm{E}+06$ & $4.84 \mathrm{E}-03$ & $4.76 \mathrm{E}-03$ \\
\hline TY-105 & $1.00 \mathrm{E}+04$ & $1.91 \mathrm{E}+06$ & $5.01 \mathrm{E}+02$ & $3.56 \mathrm{E}+03$ & $3.18 \mathrm{E}+04$ & $1.91 \mathrm{E}+06$ & $1.59 \mathrm{E}+03$ & $1.15 \mathrm{E}+04$ & $1.92 \mathrm{E}+06$ & $1.95 E+06$ & $4.84 \mathrm{E}-03$ & $4.76 \mathrm{E}-03$ \\
\hline TY-106 & $1.00 \mathrm{E}+04$ & $1.91 \mathrm{E}+06$ & $5.01 \mathrm{E}+02$ & $3.56 \mathrm{E}+03$ & $3.18 \mathrm{E}+04$ & $1.91 \mathrm{E}+06$ & $1.59 \mathrm{E}+03$ & $1.15 \mathrm{E}+04$ & $1.92 \mathrm{E}+06$ & $1.95 \mathrm{E}+06$ & $4.84 \mathrm{E}-03$ & $4.76 \mathrm{E}-03$ \\
\hline U-101 & $1.00 \mathrm{E}+04$ & $1.91 \mathrm{E}+06$ & $5.01 \mathrm{E}+02$ & $3.37 \mathrm{E}+03$ & $3.18 \mathrm{E}+04$ & $1.91 \mathrm{E}+06$ & $1.59 \mathrm{E}+03$ & $1.09 \mathrm{E}+04$ & $1.92 \mathrm{E}+06$ & $1.95 \mathrm{E}+06$ & $4.89 \mathrm{E}-03$ & $4.82 \mathrm{E}-03$ \\
\hline U-102 & $1.00 \mathrm{E}+04$ & $1.91 \mathrm{E}+06$ & $5.01 \mathrm{E}+02$ & $3.37 \mathrm{E}+03$ & $3.18 \mathrm{E}+04$ & $1.91 \mathrm{E}+06$ & $1.59 \mathrm{E}+03$ & $1.09 \mathrm{E}+04$ & $1.92 \mathrm{E}+06$ & $1.95 \mathrm{E}+06$ & $4.89 \mathrm{E}-03$ & $4.82 \mathrm{E}-03$ \\
\hline U-103 & $1.00 \mathrm{E}+04$ & $1.91 \mathrm{E}+06$ & $5.01 \mathrm{E}+02$ & $3.37 \mathrm{E}+03$ & $3.18 \mathrm{E}+04$ & $1.91 \mathrm{E}+06$ & $1.59 \mathrm{E}+03$ & $1.09 \mathrm{E}+04$ & $1.92 \mathrm{E}+06$ & $1.95 \mathrm{E}+06$ & $4.89 \mathrm{E}-03$ & $4.82 \mathrm{E}-03$ \\
\hline U-104 & $1.00 \mathrm{E}+04$ & $1.91 \mathrm{E}+06$ & $5.01 \mathrm{E}+02$ & $3.37 \mathrm{E}+03$ & $3.18 \mathrm{E}+04$ & $1.91 \mathrm{E}+06$ & $1.59 \mathrm{E}+03$ & $1.09 \mathrm{E}+04$ & $1.92 \mathrm{E}+06$ & $1.95 \mathrm{E}+06$ & $4.89 \mathrm{E}-03$ & $4.82 \mathrm{E}-03$ \\
\hline U-105 & $1.00 \mathrm{E}+04$ & $1.91 \mathrm{E}+06$ & $5.01 \mathrm{E}+02$ & $3.37 \mathrm{E}+03$ & $3.18 \mathrm{E}+04$ & $1.91 \mathrm{E}+06$ & $1.59 \mathrm{E}+03$ & $1.09 \mathrm{E}+04$ & $1.92 \mathrm{E}+06$ & $1.95 \mathrm{E}+06$ & $4.89 \mathrm{E}-03$ & $4.82 \mathrm{E}-03$ \\
\hline U-106 & $1.00 \mathrm{E}+04$ & $1.91 \mathrm{E}+06$ & $5.01 \mathrm{E}+02$ & $3.37 \mathrm{E}+03$ & $3.18 \mathrm{E}+04$ & $1.91 \mathrm{E}+06$ & $1.59 \mathrm{E}+03$ & $1.09 \mathrm{E}+04$ & $1.92 \mathrm{E}+06$ & $1.95 \mathrm{E}+06$ & $4.89 \mathrm{E}-03$ & $4.82 \mathrm{E}-03$ \\
\hline U-107 & $1.00 \mathrm{E}+04$ & $1.91 \mathrm{E}+06$ & $5.01 \mathrm{E}+02$ & $3.37 \mathrm{E}+03$ & $3.18 \mathrm{E}+04$ & $1.91 \mathrm{E}+06$ & $1.59 \mathrm{E}+03$ & $1.09 \mathrm{E}+04$ & $1.92 \mathrm{E}+06$ & $1.95 \mathrm{E}+06$ & $4.89 \mathrm{E}-03$ & $4.82 \mathrm{E}-03$ \\
\hline U-108 & $1.00 \mathrm{E}+04$ & $1.91 E+06$ & $5.01 \mathrm{E}+02$ & $3.37 \mathrm{E}+03$ & $3.18 \mathrm{E}+04$ & $1.91 \mathrm{E}+06$ & $1.59 \mathrm{E}+03$ & $1.09 \mathrm{E}+04$ & $1.92 \mathrm{E}+06$ & $1.95 \mathrm{E}+06$ & $4.89 \mathrm{E}-03$ & $4.82 \mathrm{E}-03$ \\
\hline U-109 & $1.00 \mathrm{E}+04$ & $1.91 \mathrm{E}+06$ & $5.01 \mathrm{E}+02$ & $3.37 \mathrm{E}+03$ & $3.18 \mathrm{E}+04$ & $1.91 \mathrm{E}+06$ & $1.59 \mathrm{E}+03$ & $1.09 \mathrm{E}+04$ & $1.92 \mathrm{E}+06$ & $1.95 \mathrm{E}+06$ & $4.89 \mathrm{E}-03$ & $4.82 \mathrm{E}-03$ \\
\hline U-110 & $1.00 \mathrm{E}+04$ & $1.91 \mathrm{E}+06$ & $5.01 \mathrm{E}+02$ & $3.37 \mathrm{E}+03$ & $3.18 \mathrm{E}+04$ & $1.91 \mathrm{E}+06$ & $1.59 \mathrm{E}+03$ & $1.09 \mathrm{E}+04$ & $1.92 \mathrm{E}+06$ & $1.95 \mathrm{E}+06$ & $4.89 \mathrm{E}-03$ & $4.82 \mathrm{E}-03$ \\
\hline U-111 & $1.00 \mathrm{E}+04$ & $1.91 \mathrm{E}+06$ & $5.01 \mathrm{E}+02$ & $3.37 \mathrm{E}+03$ & $3.18 \mathrm{E}+04$ & $1.91 E+06$ & $1.59 \mathrm{E}+03$ & $1.09 \mathrm{E}+04$ & $1.92 \mathrm{E}+06$ & $1.95 \mathrm{E}+06$ & $4.89 \mathrm{E}-03$ & $4.82 \mathrm{E}-03$ \\
\hline U-112 & $1.00 \mathrm{E}+04$ & $1.91 \mathrm{E}+06$ & $5.01 \mathrm{E}+02$ & $3.37 \mathrm{E}+03$ & $3.18 \mathrm{E}+04$ & $1.91 \mathrm{E}+06$ & $1.59 \mathrm{E}+03$ & $1.09 \mathrm{E}+04$ & $1.92 \mathrm{E}+06$ & $1.95 \mathrm{E}+06$ & $4.89 \mathrm{E}-03$ & $4.82 \mathrm{E}-03$ \\
\hline $\mathrm{U}-201$ & $8.02 \mathrm{E}+03$ & $1.91 \mathrm{E}+06$ & $0.00 \mathrm{E}+00$ & $2.79 \mathrm{E}+03$ & $2.54 \mathrm{E}+04$ & $1.91 \mathrm{E}+06$ & $0.00 \mathrm{E}+00$ & $9.05 \mathrm{E}+03$ & $1.92 \mathrm{E}+06$ & $1.94 \mathrm{E}+06$ & $1.61 \mathrm{E}-04$ & $1.60 \mathrm{E}-04$ \\
\hline U-202 & $8.02 \mathrm{E}+03$ & $1.91 \mathrm{E}+06$ & $0.00 \mathrm{E}+00$ & $2.79 \mathrm{E}+03$ & $2.54 E+04$ & $1.91 E+06$ & $0.00 \mathrm{E}+00$ & $9.05 \mathrm{E}+03$ & $1.92 \mathrm{E}+06$ & $1.94 \mathrm{E}+06$ & $1.61 \mathrm{E}-04$ & $1.60 \mathrm{E}-04$ \\
\hline U-203 & $8.02 \mathrm{E}+03$ & $1.91 \mathrm{E}+06$ & $0.00 \mathrm{E}+00$ & $2.79 \mathrm{E}+03$ & $2.54 \mathrm{E}+04$ & $1.91 \mathrm{E}+06$ & $0.00 \mathrm{E}+00$ & $9.05 \mathrm{E}+03$ & $1.92 \mathrm{E}+06$ & $1.94 \mathrm{E}+06$ & $1.61 \mathrm{E}-04$ & $1.60 \mathrm{E}-04$ \\
\hline U-204 & $8.02 E+03$ & $1.91 \mathrm{E}+06$ & $0.00 \mathrm{E}+00$ & $2.79 \mathrm{E}+03$ & $2.54 \mathrm{E}+04$ & $1.91 \mathrm{E}+06$ & $0.00 \mathrm{E}+00$ & $9.05 \mathrm{E}+03$ & $1.92 \mathrm{E}+06$ & $1.94 \mathrm{E}+06$ & $1.61 \mathrm{E}-04$ & $1.60 \mathrm{E}-04$ \\
\hline
\end{tabular}


RPP-5926 REV 9

APPENDIX C

AMMONIA CONTRIBUTION TO FLAMMABILITY

C-1 


\section{C1.0 PURPOSE}

This appendix establishes a reasonably conservative ammonia contribution to flammability for double-shell tank (DST) and single-shell tank (SST) time to the lower flammability limit (TTLFL) calculations.

\section{C2.0 BACKGROUND}

Headspace flammability is dominated by hydrogen, and although a gas mixture of several fuel components (e.g., methane, hydrocarbons, ammonia) can exist, the mixture behaves like a hydrogen-air system with respect to flammability (RPP-7771). The previous approach for ammonia contribution to flammability was to assume a constant release rate (as determined by Equation C-1) and include this contribution along with hydrogen and methane generation.

Where

$$
R_{g}=h \cdot A \cdot\left(C_{l}-K_{H} \cdot P_{g}\right)
$$

$\mathrm{R}_{\mathrm{g}}=$ ammonia release rate, $\mathrm{kg}$-mole/s

$\mathrm{h}=$ overall mass transport coefficient from liquid to vapor, $\mathrm{m} / \mathrm{s}$

$\mathrm{A}=$ effective transport area, $\mathrm{m}^{2}$

$\mathrm{C}_{\mathrm{l}}=$ current ammonia concentration in liquid, $\mathrm{kg}-\mathrm{mole} / \mathrm{m}^{3}$

$\mathrm{K}_{\mathrm{H}}=$ Henry's Law constant, $\mathrm{kg}-\mathrm{mole} / \mathrm{m}^{3}$-atm

$\mathrm{P}_{\mathrm{g}}=$ ammonia partial pressure, atm

The product $\mathrm{h} \cdot \mathrm{A}$ was estimated through Equation $\mathrm{C}-2$.

Where

$$
h \cdot A=\left[\frac{C_{g}^{s s} \cdot V_{r}}{\left(C_{l}-K_{H} \cdot P_{g}\right)}\right]
$$

$$
\begin{aligned}
\mathrm{C}_{\mathrm{g}}^{\mathrm{ss}} & =\text { steady-state headspace ammonia concentration, } \mathrm{kg}-\mathrm{mole} / \mathrm{m}^{3} \\
\mathrm{~V}_{\mathrm{r}} & =\text { ventilation rate, } \mathrm{m}^{3} / \mathrm{s}
\end{aligned}
$$

Steady-state ammonia concentration $\left(\mathrm{C}_{\mathrm{g}}^{\mathrm{ss}}\right)$, headspace ventilation rate $\left(\mathrm{V}_{\mathrm{r}}\right)$, and ammonia liquid concentration $\left(\mathrm{C}_{\mathrm{l}}\right)$ must be known simultaneously for current conditions to accurately estimate the product $h \cdot A$. Estimates of $h \cdot A$ were based on the best available information; however, these data are not routinely collected and the available data in some cases do not accurately reflect current waste tank conditions. To remedy this, a reasonably conservative ammonia flammability contribution for use in TTLFL calculations is developed here. 


\section{C3.0 AMMONIA DATA}

Ammonia is of interest in Tank Farms as a flammable gas hazard and noxious vapor hazard so a significant amount of data exists. This section reviews data collected over more than 15 years and examines mass transfer characteristics that will help determine a reasonably conservative ammonia contribution to flammability.

\section{C3.1 MEASURED AMMONIA CONCENTRATIONS}

Ammonia concentrations have been measured at the source (i.e., tank headspace or ventilation stack) more than 1,500 times. Most measurements were made under quiescent waste conditions, but data have also been collected during waste disturbing activities.

\section{C3.1.1 Quiescent Waste Conditions}

Table C-1 shows the maximum ammonia concentration measured for each source and when this maximum occurred. Slightly more than $70 \%$ of the tank headspaces (127 of 177) have been analyzed for ammonia. Sampling was targeted at those tanks that were most likely to contain noxious vapors, so ammonia concentrations in the 50 non-sampled tanks are expected to be lower.

Table C-1 data are sorted from the highest to lowest measured concentration. Tank 241-U-103 has the highest measured concentration, $2502 \mathrm{ppm}$ or $1.67 \%$ of the LFL.

Table C-1. Maximum Measured Concentrations.*

\begin{tabular}{|c|c|c|c|c|}
\hline $\begin{array}{c}\text { Tank } \\
\text { Name }\end{array}$ & $\begin{array}{c}\text { Sample } \\
\text { Location }\end{array}$ & $\begin{array}{c}\text { Maximum Measured } \\
\text { Concentration } \\
\text { (ppmv) }\end{array}$ & $\begin{array}{c}\text { Maximum Measured } \\
\text { Concentration } \\
\text { (\% of LFL) }\end{array}$ & $\begin{array}{c}\text { Sample } \\
\text { Date }\end{array}$ \\
\hline $241-U-103$ & Headspace & 2502 & $1.67 \%$ & $12 / 15 / 2004$ \\
\hline $241-U-105$ & Headspace & 2383 & $1.59 \%$ & $12 / 27 / 2004$ \\
\hline $241-U-102$ & Headspace & 2046 & $1.36 \%$ & $11 / 29 / 2004$ \\
\hline $241-U-108$ & Headspace & 1216 & $0.81 \%$ & $11 / 28 / 2004$ \\
\hline $241-$ BY-108 & Headspace & 1140 & $0.76 \%$ & $10 / 27 / 1994$ \\
\hline $241-U-106$ & Headspace & 1013 & $0.68 \%$ & $3 / 7 / 1995$ \\
\hline $241-$ TX-110 & Headspace & 1000 & $0.67 \%$ & $9 / 23 / 1997$ \\
\hline $241-$ BY-107 & Headspace & 978 & $0.65 \%$ & $10 / 26 / 1994$ \\
\hline $241-U-101$ & Headspace & 949 & $0.63 \%$ & $1 / 26 / 2005$ \\
\hline $241-S-101$ & Headspace & 940 & $0.63 \%$ & $2 / 1 / 2005$ \\
\hline $241-$ S-102 & Headspace & 805 & $0.54 \%$ & $9 / 19 / 1996$ \\
\hline $241-$ A-101 & Headspace & 800 & $0.53 \%$ & $6 / 8 / 1995$ \\
\hline $241-U-110$ & Headspace & 728 & $0.49 \%$ & $12 / 20 / 2004$ \\
\hline $241-U-111$ & Headspace & 682 & $0.45 \%$ & $2 / 28 / 1995$ \\
\hline $241-$ TX-111 & Headspace & 638 & $0.43 \%$ & $10 / 12 / 1995$ \\
\hline $241-U-109$ & Headspace & 608 & $0.41 \%$ & $8 / 10 / 1995$ \\
\hline $241-\mathrm{C}-104$ & Headspace & 561 & $0.37 \%$ & $6 / 15 / 2004$ \\
\hline
\end{tabular}


Table C-1. Maximum Measured Concentrations.*

\begin{tabular}{|c|c|c|c|c|}
\hline $\begin{array}{c}\text { Tank } \\
\text { Name }\end{array}$ & $\begin{array}{c}\text { Sample } \\
\text { Location }\end{array}$ & $\begin{array}{c}\text { Maximum Measured } \\
\text { Concentration } \\
\text { (ppmv) }\end{array}$ & $\begin{array}{c}\text { Maximum Measured } \\
\text { Concentration } \\
\text { (\% of LFL) }\end{array}$ & $\begin{array}{c}\text { Sample } \\
\text { Date }\end{array}$ \\
\hline 241-SY-102 & Headspace & $551 \uparrow$ & $0.37 \%$ & $9 / 12 / 2000$ \\
\hline 241-U-107 & Headspace & 474 & $0.32 \%$ & $2 / 17 / 1995$ \\
\hline SY Ventilation & Vent Stack & $442 \uparrow$ & $0.29 \%$ & $8 / 30 / 2000$ \\
\hline $241-\mathrm{BY}-110$ & Headspace & 426 & $0.28 \%$ & $11 / 11 / 1994$ \\
\hline 241-C-103 & Headspace & 349 & $0.23 \%$ & $5 / 20 / 1994$ \\
\hline $241-U-112$ & Headspace & 326 & $0.22 \%$ & $7 / 9 / 1996$ \\
\hline $241-C-102$ & Headspace & 317 & $0.21 \%$ & $6 / 24 / 2004$ \\
\hline 241-BY-104 & Headspace & 286 & $0.19 \%$ & $4 / 22 / 1994$ \\
\hline 241-A-106 & Headspace & 280 & $0.19 \%$ & $1 / 16 / 1997$ \\
\hline 241-A-103 & Headspace & 273 & $0.18 \%$ & $11 / 9 / 1995$ \\
\hline $241-S-107$ & Headspace & 270 & $0.18 \%$ & $2 / 7 / 2005$ \\
\hline 241-BX-104 & Headspace & 269 & $0.18 \%$ & $8 / 22 / 1996$ \\
\hline $241-A-102$ & Headspace & 263 & $0.18 \%$ & $11 / 10 / 1995$ \\
\hline 241-AP-104 & Headspace & 260 & $0.17 \%$ & $11 / 28 / 2000$ \\
\hline 241-TX-106 & Headspace & 260 & $0.17 \%$ & $3 / 5 / 1997$ \\
\hline 241-AP-102 & Headspace & 234 & $0.16 \%$ & $3 / 28 / 2001$ \\
\hline $241-\mathrm{T}-111$ & Headspace & 227 & $0.15 \%$ & $1 / 20 / 1995$ \\
\hline 241-AN-101 & Headspace & 219 & $0.15 \%$ & $4 / 24 / 2001$ \\
\hline 241-AP-106 & Headspace & 197 & $0.13 \%$ & $5 / 8 / 2001$ \\
\hline 241-SX-106 & Headspace & 188 & $0.13 \%$ & $3 / 24 / 1995$ \\
\hline 241-BY-102 & Headspace & 180 & $0.12 \%$ & $11 / 21 / 1995$ \\
\hline 241-TX-102 & Headspace & 170 & $0.11 \%$ & $10 / 13 / 1997$ \\
\hline 241-TX-114 & Headspace & 170 & $0.11 \%$ & $3 / 25 / 1997$ \\
\hline 241-BX-105 & Headspace & 155 & $0.10 \%$ & $4 / 24 / 1996$ \\
\hline $241-C-110$ & Headspace & 154 & $0.10 \%$ & $6 / 9 / 2004$ \\
\hline $241-S-103$ & Headspace & 153 & $0.10 \%$ & $6 / 12 / 1996$ \\
\hline $241-S-110$ & Headspace & 153 & $0.10 \%$ & $12 / 5 / 1995$ \\
\hline 241-T-107 & Headspace & 127 & $0.08 \%$ & $1 / 18 / 1995$ \\
\hline $241-S-111$ & Headspace & 124 & $0.08 \%$ & $3 / 21 / 1995$ \\
\hline 241-TX-112 & Headspace & 120 & $0.08 \%$ & $12 / 9 / 1997$ \\
\hline 241-T-104 & Headspace & 110 & $0.07 \%$ & $2 / 7 / 1996$ \\
\hline 241-TX-103 & Headspace & 110 & $0.07 \%$ & $10 / 24 / 1997$ \\
\hline 241-T-110 & Headspace & 109 & $0.07 \%$ & $8 / 31 / 1995$ \\
\hline $241-C-101$ & Headspace & 99 & $0.07 \%$ & $9 / 1 / 1994$ \\
\hline 241-BX-103 & Headspace & 96 & $0.06 \%$ & $8 / 1 / 1996$ \\
\hline 241-BY-106 & Headspace & 92 & $0.06 \%$ & $5 / 4 / 1994$ \\
\hline 241-S-112 & Headspace & 90 & $0.06 \%$ & $7 / 11 / 1995$ \\
\hline 241-BX-107 & Headspace & 89 & $0.06 \%$ & $11 / 17 / 1995$ \\
\hline $241-C-107$ & Headspace & 87 & $0.06 \%$ & $9 / 29 / 1994$ \\
\hline 241-SX-103 & Headspace & 80 & $0.05 \%$ & $3 / 23 / 1995$ \\
\hline 241-AP-101 & Headspace & 72 & $0.05 \%$ & $7 / 10 / 2001$ \\
\hline 241-BY-112 & Headspace & 71 & $0.05 \%$ & $11 / 18 / 1994$ \\
\hline 241-BX-110 & Headspace & 66 & $0.04 \%$ & $4 / 30 / 1996$ \\
\hline 241-BY-101 & Headspace & 65 & $0.04 \%$ & $8 / 29 / 1996$ \\
\hline
\end{tabular}


Table C-1. Maximum Measured Concentrations.*

\begin{tabular}{|c|c|c|c|c|}
\hline $\begin{array}{c}\text { Tank } \\
\text { Name }\end{array}$ & $\begin{array}{c}\text { Sample } \\
\text { Location }\end{array}$ & $\begin{array}{c}\text { Maximum Measured } \\
\text { Concentration } \\
\text { (ppmv) }\end{array}$ & $\begin{array}{c}\text { Maximum Measured } \\
\text { Concentration } \\
\text { (\% of LFL) }\end{array}$ & $\begin{array}{c}\text { Sample } \\
\text { Date }\end{array}$ \\
\hline 241-BX-111 & Headspace & 64 & $0.04 \%$ & $8 / 27 / 1996$ \\
\hline 241-TY-104 & Headspace & 62 & $0.04 \%$ & $4 / 27 / 1995$ \\
\hline 241-BY-105 & Headspace & 61 & $0.04 \%$ & $5 / 9 / 1994$ \\
\hline 241-BY-111 & Headspace & 61 & $0.04 \%$ & $11 / 16 / 1994$ \\
\hline 241-TY-103 & Headspace & 54 & $0.04 \%$ & $11 / 22 / 1996$ \\
\hline $241-\mathrm{A}-105$ & Headspace & 54 & $0.04 \%$ & $3 / 6 / 2005$ \\
\hline 241-BX-106 & Headspace & 51 & $0.03 \%$ & $8 / 15 / 1996$ \\
\hline 241-S-109 & Headspace & 48 & $0.03 \%$ & $6 / 4 / 1996$ \\
\hline 241-BY-109 & Headspace & 46 & $0.03 \%$ & $9 / 22 / 1994$ \\
\hline 241-AX-101 & Headspace & 44 & $0.03 \%$ & $6 / 15 / 1995$ \\
\hline 241-AX-103 & Headspace & 44 & $0.03 \%$ & $6 / 21 / 1995$ \\
\hline 241-TX-118 & Headspace & 43 & $0.03 \%$ & $9 / 7 / 1994$ \\
\hline $241-S-106$ & Headspace & 39 & $0.03 \%$ & $6 / 13 / 1996$ \\
\hline $241-\mathrm{S}-105$ & Headspace & 38 & $0.03 \%$ & $12 / 7 / 1995$ \\
\hline 241-AX-102 & Headspace & 37 & $0.02 \%$ & $6 / 27 / 1995$ \\
\hline 241-BY-103 & Headspace & 35 & $0.02 \%$ & $5 / 5 / 1994$ \\
\hline 241-SX-105 & Headspace & 30 & $0.02 \%$ & $7 / 26 / 1995$ \\
\hline 241-TX-104 & Headspace & 30 & $0.02 \%$ & $5 / 5 / 1997$ \\
\hline $241-S-108$ & Headspace & 27 & $0.02 \%$ & $12 / 6 / 1995$ \\
\hline 241-TY-103 & Headspace & 27 & $0.02 \%$ & $8 / 5 / 1994$ \\
\hline $241-\mathrm{C}-112$ & Headspace & 26 & $0.02 \%$ & $7 / 1 / 2004$ \\
\hline 241-SX-104 & Headspace & 26 & $0.02 \%$ & $7 / 25 / 1995$ \\
\hline 241-TX-108 & Headspace & 26 & $0.02 \%$ & $12 / 5 / 1997$ \\
\hline 241-B-107 & Headspace & 26 & $0.02 \%$ & $7 / 23 / 1996$ \\
\hline 241-TX-113 & Headspace & 24 & $0.02 \%$ & $8 / 6 / 1997$ \\
\hline $241-\mathrm{T}-101$ & Headspace & 23 & $0.02 \%$ & $12 / 1 / 1997$ \\
\hline 241-TX-105 & Headspace & 21 & $0.01 \%$ & $12 / 20 / 1994$ \\
\hline 241-SX-109 & Headspace & 18 & $0.01 \%$ & $8 / 1 / 1995$ \\
\hline 241-TY-101 & Headspace & 18 & $0.01 \%$ & $8 / 5 / 1994$ \\
\hline 241-SX-102 & Headspace & 16 & $0.01 \%$ & $7 / 19 / 1995$ \\
\hline 241-TX-116 & Headspace & 15 & $0.01 \%$ & $9 / 18 / 1997$ \\
\hline 241-C-109 & Headspace & 11 & $0.01 \%$ & $8 / 10 / 1994$ \\
\hline 241-B-103 & Headspace & 10 & $0.01 \%$ & $2 / 8 / 1995$ \\
\hline $241-C-106$ & Headspace & 8.8 & $0.01 \%$ & $2 / 15 / 1994$ \\
\hline 241-C-111 & Headspace & 6.8 & $0.00 \%$ & $9 / 13 / 1994$ \\
\hline 241-B-105 & Headspace & 5.9 & $0.00 \%$ & $7 / 30 / 1996$ \\
\hline 241-B-102 & Headspace & 5.5 & $0.00 \%$ & $4 / 18 / 1996$ \\
\hline 241-SX-101 & Headspace & 4.2 & $0.00 \%$ & $7 / 21 / 1995$ \\
\hline 241-TY-102 & Headspace & 4.1 & $0.00 \%$ & $4 / 12 / 1996$ \\
\hline 241-C-108 & Headspace & 3.3 & $0.00 \%$ & $8 / 5 / 1994$ \\
\hline 241-B-202 & Headspace & 3.0 & $0.00 \%$ & $7 / 18 / 1996$ \\
\hline $241-\mathrm{BX}-102$ & Headspace & 3.0 & $0.00 \%$ & $7 / 31 / 1996$ \\
\hline 241-C-105 & Headspace & 2.7 & $0.00 \%$ & $2 / 16 / 1994$ \\
\hline $241-U-201$ & Headspace & 1.8 & $0.00 \%$ & $11 / 19 / 2004$ \\
\hline
\end{tabular}


Table C-1. Maximum Measured Concentrations.*

\begin{tabular}{|c|c|c|c|c|}
\hline $\begin{array}{c}\text { Tank } \\
\text { Name } \\
\end{array}$ & $\begin{array}{c}\text { Sample } \\
\text { Location }\end{array}$ & $\begin{array}{c}\text { Maximum Measured } \\
\text { Concentration } \\
\text { (ppmv) }\end{array}$ & $\begin{array}{c}\text { Maximum Measured } \\
\text { Concentration } \\
(\% \text { of LFL) }\end{array}$ & $\begin{array}{c}\text { Sample } \\
\text { Date }\end{array}$ \\
\hline 241-U-203 & Headspace & 1.2 & $0.00 \%$ & $11 / 23 / 2004$ \\
\hline 241-AY-102 & Headspace & 1.0 & $0.00 \%$ & $3 / 18 / 2004$ \\
\hline 241-C-204 & Headspace & 0.8 & $0.00 \%$ & $7 / 2 / 1996$ \\
\hline 241-A-104 & Headspace & 0.7 & $0.00 \%$ & $3 / 2 / 2005$ \\
\hline 241-S-104 & Headspace & 0.7 & $0.00 \%$ & $2 / 14 / 2005$ \\
\hline 241-C-203 & Headspace & 0.6 & $0.00 \%$ & $5 / 12 / 2004$ \\
\hline $241-U-202$ & Headspace & 0.6 & $0.00 \%$ & $11 / 19 / 2004$ \\
\hline 241-U-204 & Headspace & 0.2 & $0.00 \%$ & $8 / 8 / 1995$ \\
\hline 241-AN-106 & Headspace & $<14$ & NA & $6 / 21 / 2001$ \\
\hline 241-AX-104 & Headspace & $<14$ & NA & $1 / 23 / 1997$ \\
\hline $241-C-201$ & Headspace & $<1.4$ & NA & $6 / 19 / 1996$ \\
\hline 241-C-202 & Headspace & $<0.7$ & NA & $6 / 25 / 1996$ \\
\hline 241-SX-107 & Headspace & $<14$ & NA & $5 / 19 / 1997$ \\
\hline 241-SX-108 & Headspace & $<14$ & NA & $7 / 16 / 1997$ \\
\hline 241-SX-110 & Headspace & $<14$ & NA & $7 / 30 / 1997$ \\
\hline 241-SX-111 & Headspace & $<14$ & NA & $5 / 22 / 1997$ \\
\hline 241-SX-112 & Headspace & $<14$ & NA & $6 / 18 / 1997$ \\
\hline 241-SX-114 & Headspace & $<14$ & NA & $6 / 25 / 1997$ \\
\hline 241-TX-101 & Headspace & $<14$ & NA & $10 / 28 / 1997$ \\
\hline 241-TX-115 & Headspace & $<14$ & NA & $11 / 20 / 1997$ \\
\hline 241-TX-117 & Headspace & $<14$ & NA & $12 / 11 / 1997$ \\
\hline 241-TY-105 & Headspace & $<14$ & NA & $10 / 20 / 1997$ \\
\hline 241-U-104 & Headspace & $<0.71$ & NA & $7 / 16 / 1996$ \\
\hline AN Ventilation & Vent Stack & $<14$ & NA & $7 / 27 / 2000$ \\
\hline AP Ventilation & Vent Stack & $<14$ & NA & $6 / 22 / 2000$ \\
\hline AW Ventilation & Vent Stack & $<14$ & NA & $6 / 14 / 2000$ \\
\hline AY/AZ Vent Sys & Vent Stack & $<14$ & NA & $8 / 14 / 2000$ \\
\hline
\end{tabular}

Note: * Data from Tank Waste Information Network (TWINS), Queried 08/14/2008, [Vapor, Headspace Sample Analysis, Analysis Results], http://twins.pnl.gov/twins.htm.

$\dagger$ Data represent transient peak from remediation of 241-SY-101.

NA $=$ Not Available

In addition to the data shown in Table C-1, 241-SY-101 ammonia concentrations were monitored by an E+ Standard Hydrogen Monitoring System. Headspace concentrations ranged between 200 to $2000 \mathrm{ppm}$ and reached a peak of $2700 \mathrm{ppm}$ during a ventilation outage in May 2001. Tank 241-SY-101 still had a significant crust layer covering $75 \%$ of the waste surface at this time and the supernatant obviously still contained significant ammonia. Since then, 241-SY-101 was returned to active service and many waste transfers have passed through the tank. The 241-SY-101 liquid waste no longer contains the high ammonia concentrations it held before and immediately after remediation.

Ammonia contribution to flammability under normal conditions is small with all maximums being less than $2 \%$ of the LFL. Statistical analysis of tank headspace vapor data (RPP-21972) showed ammonia temporal variances (i.e., the variability in headspace concentrations measured 
in samples collected on different dates) are low. Average temporal variance was only $36 \%$ and the maximum observed variance in tanks under passive ventilation was 106\% (Tank 241-S-103). Temporal variabilities were similar regardless whether samples were taken six months or more than five years apart. Results suggest that the maximums shown in Table C-1 are reasonably conservative and are only modestly influenced by the slowly changing tank conditions.

\section{C3.1.2 Waste Disturbing Activities}

In addition to the data shown in Table C-1, direct reading instruments have been used to measure ammonia during waste disturbing activities. The highest ammonia concentration ever measured was $7,400 \mathrm{ppm}(\sim 4.9 \%$ of the LFL) in the $241-\mathrm{SY}-102$ headspace during a transfer from 241-SY-101 in February 2000 (PNNL-13933). Liquid waste containing entrained air (from the 241-SY-102 drop leg siphon break) was injected into the 241-SY-102 supernatant layer. This relatively high ammonia release was a result of mixing and stripping by entrained air bubbles. Ammonia concentration quickly dropped from this peak measurement and was about 3,500 ppm $(\sim 2.3 \%$ of the LFL) after 24 hours. Ammonia concentration in 241-SY-101 peaked at $2,000 \mathrm{ppm}(\sim 1.3 \%$ of the LFL) during this transfer and quickly dropped to about $100 \mathrm{ppm}$ $(<0.1 \%$ of the LFL) after 24 hours.

The next highest measurement was 4,000 ppm ( $2.7 \%$ of the LFL) during saltwell pumping of tank 241-U-105 (PNNL-13933). Ammonia headspace concentrations slowly increased from about 2,000 ppm to about 4,000 ppm from January 21 to April 3, 2000, as the higher concentration ammonia solution towards the bottom of the tank was exposed to the headspace. From Table C-1, Tank 241-U-105 ammonia headspace concentration was 2,583 ppm on December 27, 2004, a slightly higher quiescent concentration than before saltwell pumping.

Negligible ammonia was released during Tank 241-S-106 saltwell pumping and headspace measurements failed to exceed detection limits. Tank 241-A-101 headspace ammonia concentrations were about 800 ppm before saltwell pumping began, and stayed a relatively constant $1,500 \mathrm{ppm}$ during saltwell pumping in late 2002. No headspace measurements have been made since saltwell pumping was completed.

The available ammonia data taken during waste disturbing activities suggest that ammonia concentrations can spike temporarily, before returning to concentrations near steady-state quiescent conditions. Concentrations are always limited to equilibrium with the maximum concentration found in the waste liquid (see next Section C3.2).

\section{C3.2 THEORETICAL MAXIMUM (EQUILIBRIUM) CONCENTRATIONS}

The partial pressure of ammonia over a dilute solution at equilibrium can be approximated through Henry's Law (see Equation C-3). Liquid ammonia concentrations have been measured in 56 tanks and Henry's law constants can be estimated from values in the open literature and simulant testing performed at the Hanford Site. 
Where

$$
P_{g}=\frac{C_{l}}{K_{H}}
$$

$\mathrm{P}_{\mathrm{g}}=$ ammonia partial pressure, atm

$\mathrm{C}_{\mathrm{l}}=$ ammonia concentration in the liquid, $\mathrm{kg}-\mathrm{mole} / \mathrm{m}^{3}$

$\mathrm{K}_{\mathrm{H}}=$ Henry's Law constant, $\mathrm{kg}$-mole $/ \mathrm{m}^{3}$-atm

\section{C3.2.1 Estimating Henry's Law Constants for Ammonia}

The Henry's Law constant is a function of temperature in pure water. In mixed salt solutions, Henry's Law constants are functions of both temperature and the concentrations of the ions in the solution. "The Estimation of Gas Solubility in Salt Solutions at Temperature from $273 \mathrm{~K}$ to $363 \mathrm{~K}$ " (Weisenberger and Schumpe 1996) provides a formula to calculate Henry's Law constants (shown in Equation C-4).

Where

$$
\mathrm{K}_{\mathrm{H}}=\mathrm{K}_{\mathrm{H}}^{0} \times 10^{-\Sigma_{\mathrm{i}}\left(\mathrm{h}_{\mathrm{i}}+\mathrm{h}_{\mathrm{g}}\right) \mathrm{C}_{\mathbf{i}}}
$$

$$
\begin{aligned}
\mathrm{K}_{\mathrm{H}} & =\text { Henry's Law constant in salt solution, } \mathrm{kg}-\mathrm{mole} / \mathrm{m}^{3} \text {-atm } \\
\mathrm{K}_{\mathrm{H}}^{0} & =\text { Henry's Law constant in the pure water, } \mathrm{kg}-\mathrm{mole} / \mathrm{m}^{3} \text {-atm } \\
\mathrm{h}_{\mathrm{i}} & =\text { ionic dependent coefficient, } \mathrm{m}^{3} / \mathrm{kg}-\mathrm{mole} \\
\mathrm{h}_{\mathrm{g}} & =\text { gas specific constant for ammonia, } \mathrm{m}^{3} / \mathrm{kg}-\mathrm{mole} \\
\mathrm{C}_{\mathrm{i}} & =\text { ion concentration in the solution, } \mathrm{kg}-\mathrm{mole} / \mathrm{m}^{3}
\end{aligned}
$$

An expression for $\mathrm{K}_{\mathrm{H}}^{0}$ as a function of temperature is given in Clegg and Brimblecombe (1989) and is shown in Equation C-5. Equation C-5 results are for ammonia dissolved in pure water and have units of mole $/ \mathrm{kg}$ of water-atm, which is approximately equal to $\mathrm{kg}-\mathrm{mole} / \mathrm{m}^{3}$-atm over the temperature range and ammonia concentrations of interest.

Where

$$
\mathrm{K}_{\mathrm{H}}^{0}=\mathrm{e}^{-8.0964+3917.50 / \mathrm{T}-0.00314 \mathrm{~T}}
$$

$$
\begin{aligned}
\mathrm{K}_{\mathrm{H}}^{0} & =\text { Henry's Law constant in the pure water, mole } / \mathrm{kg} \text { of water-atm } \\
\mathrm{T} & =\text { temperature, } \mathrm{K}
\end{aligned}
$$

\section{C3.2.2 Correction Factors for High Ionic Strength Solutions}

The Weisenberger and Schumpe (1996) formula (Equation C-4) is accurate for solutions up to 5 molar, and is known to under-predict ammonia solubility at higher concentrations. Simulant experiments performed at Hanford (RPP-4941) examined Henry's Law constants for higher ionic strengths. The study covered solution concentrations ranging from 5 to 20 molar at temperatures of $21^{\circ} \mathrm{C}$ to $70^{\circ} \mathrm{C}$. Henry's Law correction factors as a function of temperature and ionic strength are given in Table C-2 and plotted in Figure C-1 (RPP-5926 Figure C-1.xls, SVF-1549). 
Table C-2. Ratio of Measured Henry's Law Constant to Calculated Henry's Law Constant at Various Ionic Strengths and Temperatures.*

\begin{tabular}{|c|c|c|c|c|c|c|}
\hline $\begin{array}{c}\text { Ionic Strength } \\
\left(\mathrm{kg}-\mathrm{mole} / \mathrm{m}^{3}\right)\end{array}$ & $\begin{array}{l}\mathrm{K}_{\text {meas }} / \mathrm{K}_{\text {calc }} \\
\text { at } 21.5^{\circ} \mathrm{C}\end{array}$ & $\begin{array}{c}\mathrm{K}_{\text {meas }} / \mathrm{K}_{\text {calc }} \\
\text { at } 30^{\circ} \mathrm{C}\end{array}$ & $\begin{array}{c}\mathrm{K}_{\text {meas }} / \mathrm{K}_{\text {calc }} \\
\text { at } 40^{\circ} \mathrm{C}\end{array}$ & $\begin{array}{c}\mathrm{K}_{\text {meas }} / \mathrm{K}_{\text {calc }} \\
\text { at } 50^{\circ} \mathrm{C}\end{array}$ & $\begin{array}{c}\mathrm{K}_{\text {meas }} / \mathrm{K}_{\text {calc }} \\
\text { at } 60^{\circ} \mathrm{C}\end{array}$ & $\begin{array}{c}\mathbf{K}_{\text {meas }} / K_{\text {calc }} \\
\text { at } 70^{\circ} \mathbf{C}\end{array}$ \\
\hline 20 & 2.17 & 2.38 & 2.42 & 2.85 & 3.68 & 4.96 \\
\hline 15 & 1.52 & 1.68 & 2.17 & 2.41 & 2.67 & 3.34 \\
\hline 10 & 1.33 & 1.46 & 1.53 & 1.76 & 1.94 & 2.01 \\
\hline 5 & 1.10 & 1.33 & 1.13 & 0.97 & 1.61 & 1.55 \\
\hline
\end{tabular}

Notes: * Data from RPP-4941, Appendix H.

Figure C-1. Henry's Law Correction Factors

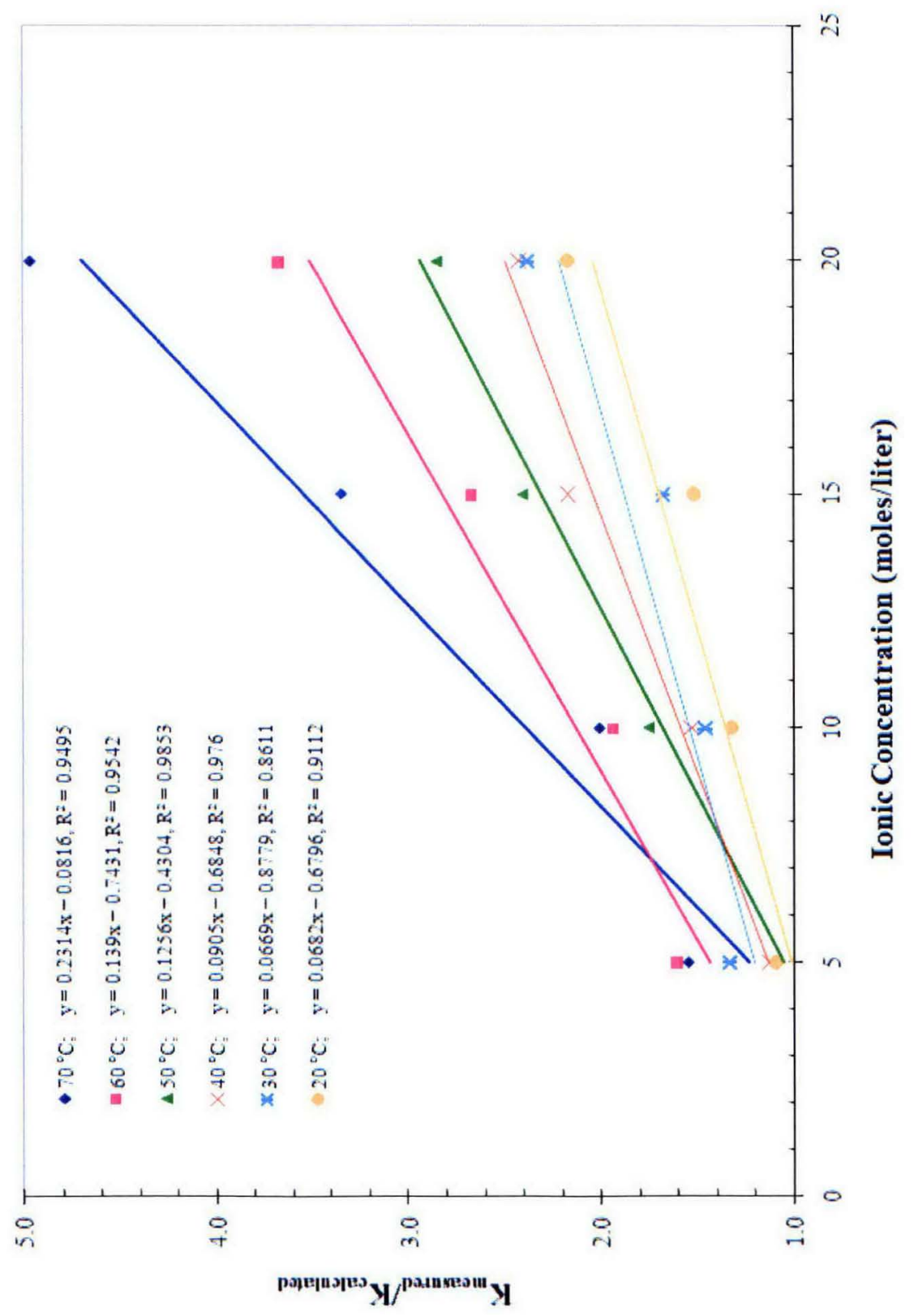

C-9 
Examining Figure C-1, the ratio between measured and calculated Henry's Law constants vary approximately linearly with increasing ionic strength. Increasing temperature appears also to increase the ratio. Linear fit equations in $10^{\circ} \mathrm{C}$ intervals are shown on the Figure $\mathrm{C}-1$ legend. Knowing waste temperatures, liquid ammonia concentrations, and ion concentrations coupled with the ionic and gas specific dependent coefficients $\left(h_{i}\right.$ and $\left.h_{g}\right)$, maximum theoretical ammonia concentrations can be calculated.

\section{C.3.2.3 Calculated Maximum Headspace Concentrations}

The linear equations shown on Figure C-1 provide correction factors to the calculated Henry's Law constant (Equation C-6).

$$
\mathrm{K}_{\mathrm{H} \text { in solution }}=\mathrm{K}_{\mathrm{H}}^{0} \times 10^{-\sum_{\mathrm{i}}\left(\mathrm{h}_{\mathrm{i}}+\mathrm{h}_{\mathrm{g}}\right) \mathrm{C}_{\mathrm{i}}} \times(\text { Correction Factor })
$$

Waste temperatures were rounded to the nearest $10{ }^{\circ} \mathrm{C}$ for selecting temperature/ionic strength correction factors shown in Figure $\mathrm{C}-1$. This was assumed to be sufficiently accurate for temperatures that fall between the $10^{\circ} \mathrm{C}$ intervals. Waste temperatures lower than $20^{\circ} \mathrm{C}$ used the temperature/ionic strength correction factors determine for $20^{\circ} \mathrm{C}$. Equilibrium ammonia concentrations were calculated in a separate spreadsheet (RPP-5926 Ammonia Evaluation.xls, SVF-1549) and results are shown in Table C-3. TTLFL results under hypothetical zero ventilation (see main text) are included in last column of Table C-3.

Table C-3. Maximum Theoretical Headspace Ammonia Concentrations.

\begin{tabular}{|c|c|c|c|c|c|}
\hline Tank & $\begin{array}{c}\mathbf{C}_{\mathbf{l}}{ }^{1} \\
\left(\mathrm{~kg}-\mathrm{mole} / \mathrm{m}^{3}\right)\end{array}$ & $\begin{array}{c}\mathrm{K}_{\mathrm{H} \text { in solution }}{ }^{1} \\
\text { (kg-mole/m } / \mathrm{m}^{3} \text {-atm) }\end{array}$ & $\begin{array}{c}\mathbf{P}_{\mathbf{g}}{ }^{1} \\
(\mathbf{a t m})\end{array}$ & $\begin{array}{l}\text { LFL }^{1} \\
(\%)\end{array}$ & $\begin{array}{c}\text { TTLFL }^{2} \\
\text { (days) }\end{array}$ \\
\hline 241-SX-103 & 0.106 & 3.5 & $3.01 \mathrm{E}-02$ & 20 & NA \\
\hline 241-SX-105 & 0.071 & 3.7 & $1.94 \mathrm{E}-02$ & 13 & NA \\
\hline 241-SX-102 & 0.080 & 4.9 & $1.66 \mathrm{E}-02$ & 11 & NA \\
\hline 241-SY-101 & $0.271^{3}$ & 18.2 & $1.49 \mathrm{E}-02$ & 10 & 1193 \\
\hline 241-SX-106 & 0.147 & 10.1 & $1.46 \mathrm{E}-02$ & 9.7 & NA \\
\hline 241-SX-108 & 0.076 & 5.3 & $1.44 \mathrm{E}-02$ & 9.6 & NA \\
\hline $241-S-102$ & 0.100 & 7.1 & $1.41 \mathrm{E}-02$ & 9.4 & $>1826$ \\
\hline $241-\mathrm{A}-101$ & 0.031 & 3.3 & $9.36 \mathrm{E}-03$ & 6.2 & $>1826$ \\
\hline 241-BY-106 & 0.060 & 7.4 & $8.13 \mathrm{E}-03$ & 5.4 & $>1826$ \\
\hline $241-S-101$ & 0.071 & 8.9 & $7.94 \mathrm{E}-03$ & 5.3 & $>1826$ \\
\hline $241-U-103$ & 0.082 & 10.8 & $7.62 \mathrm{E}-03$ & 5.1 & 1082 \\
\hline $241-U-105$ & 0.094 & 12.4 & $7.59 \mathrm{E}-03$ & 5.1 & 1093 \\
\hline 241-SY-103 & 0.076 & 10.3 & $7.38 \mathrm{E}-03$ & 4.9 & 659 \\
\hline 241-AX-101 & 0.032 & 4.7 & $6.81 \mathrm{E}-03$ & 4.5 & NA \\
\hline $241-S-111$ & 0.088 & 14.1 & $6.25 \mathrm{E}-03$ & 4.2 & $>1826$ \\
\hline $241-A-103$ & 0.031 & 5.0 & $6.19 \mathrm{E}-03$ & 4.1 & $>1826$ \\
\hline $241-S-103$ & 0.083 & 13.4 & $6.16 \mathrm{E}-03$ & 4.1 & $>1826$ \\
\hline 241-SX-104 & 0.047 & 7.9 & $5.91 \mathrm{E}-03$ & 3.9 & NA \\
\hline 241-U-108 & 0.062 & 10.9 & $5.66 \mathrm{E}-03$ & 3.8 & $>1826$ \\
\hline $241-U-109$ & 0.065 & 14.2 & $4.54 \mathrm{E}-03$ & 3.0 & $>1826$ \\
\hline $241-C-103$ & 0.071 & 17.1 & $4.16 \mathrm{E}-03$ & 2.8 & $>1826$ \\
\hline 241-AN-103 & 0.020 & 5.7 & $3.55 \mathrm{E}-03$ & 2.4 & 408 \\
\hline 241-SX-101 & 0.008 & 2.6 & $3.01 \mathrm{E}-03$ & 2.0 & NA \\
\hline $241-U-102$ & 0.044 & 15.3 & $2.86 \mathrm{E}-03$ & 1.9 & $>1826$ \\
\hline 241-U-106 & 0.062 & 21.7 & $2.85 \mathrm{E}-03$ & 1.9 & $>1826$ \\
\hline
\end{tabular}


Table C-3. Maximum Theoretical Headspace Ammonia Concentrations.

\begin{tabular}{|c|c|c|c|c|c|}
\hline Tank & $\begin{array}{c}\mathrm{C}_{\mathrm{l}}^{1} \\
\left(\mathrm{~kg}-\mathrm{mole} / \mathrm{m}^{3}\right)\end{array}$ & $\begin{array}{c}\bar{K}_{\mathrm{H} \text { in solution }}{ }^{1} \\
\text { (kg-mole/m } / \text { m }^{3} \text {-atm) }\end{array}$ & $\begin{array}{c}\mathbf{P}_{\mathbf{q}}{ }^{1} \\
\text { (atm) }\end{array}$ & $\begin{array}{l}\mathbf{L F L}^{1} \\
(\%)\end{array}$ & $\begin{array}{c}\text { TTLFL }^{2} \\
\text { (days) }\end{array}$ \\
\hline 241-SY-102 & $0.068^{4}$ & 33.6 & $2.01 \mathrm{E}-03$ & 1.3 & $>1826$ \\
\hline $241-\mathrm{AN}-104$ & 0.014 & 7.4 & $1.95 \mathrm{E}-03$ & 1.3 & 255 \\
\hline 241-AZ-102 & 0.026 & 13.3 & $1.94 \mathrm{E}-03$ & 1.3 & 53 \\
\hline $241-\mathrm{AN}-105$ & 0.012 & 7.4 & $1.68 \mathrm{E}-03$ & 1.1 & 409 \\
\hline $241-T-110$ & 0.088 & 53.3 & $1.65 \mathrm{E}-03$ & 1.1 & $>1826$ \\
\hline $241-U-107$ & 0.024 & 16.8 & $1.41 \mathrm{E}-03$ & 0.94 & $>1826$ \\
\hline $241-\mathrm{AW}-106$ & 0.021 & 18.5 & $1.12 \mathrm{E}-03$ & 0.75 & 655 \\
\hline 241-S-106 & 0.014 & 12.6 & 1.12E-03 & 0.75 & $>1826$ \\
\hline 241-AP-107 & 0.062 & 60.1 & $1.04 \mathrm{E}-03$ & 0.69 & 1488 \\
\hline 241-AP-108 & 0.047 & 49.5 & $9.49 \mathrm{E}-04$ & 0.63 & 382 \\
\hline 241-AP-105 & 0.019 & 20.5 & $9.27 \mathrm{E}-04$ & 0.62 & $>1826$ \\
\hline 241-AN-102 & 0.012 & 14.5 & $8.51 \mathrm{E}-04$ & 0.57 & 88 \\
\hline 241-AN-107 & 0.012 & 16.7 & $7.41 \mathrm{E}-04$ & 0.49 & 102 \\
\hline 241-AP-101 & 0.026 & 35.0 & $7.36 \mathrm{E}-04$ & 0.49 & 1680 \\
\hline 241-AP-102 & 0.026 & 35.3 & $7.31 \mathrm{E}-04$ & 0.49 & 806 \\
\hline $241-\mathrm{AW}-105$ & 0.059 & 83.8 & $7.08 \mathrm{E}-04$ & $0 . \overline{47}$ & $>1826$ \\
\hline 241-AZ-101 & 0.007 & 10.5 & $7.00 \mathrm{E}-04$ & 0.47 & 132 \\
\hline 241-AP-103 & 0.015 & 30.2 & $4.86 \mathrm{E}-04$ & 0.32 & 200 \\
\hline $241-\mathrm{AW}-103$ & 0.030 & 62.2 & $4.83 \mathrm{E}-04$ & 0.32 & 1101 \\
\hline 241-AY-101 & 0.007 & 20.5 & $3.24 \mathrm{E}-04$ & 0.22 & 667 \\
\hline 241-AN-106 & 0.012 & 42.9 & $2.87 \mathrm{E}-04$ & 0.19 & 459 \\
\hline 241-AY-102 & 0.004 & 19.4 & $2.24 \mathrm{E}-04$ & 0.15 & 118 \\
\hline 241-AP-104 & 0.008 & 40.0 & $1.95 \mathrm{E}-04$ & 0.13 & 797 \\
\hline $241-\mathrm{AW}-104$ & 0.010 & 62.2 & $1.60 \mathrm{E}-04$ & 0.11 & 544 \\
\hline 241-AP-106 & 0.004 & 35.8 & $1.11 \mathrm{E}-04$ & 0.07 & 558 \\
\hline $241-T-203$ & 0.006 & 54.7 & 1.07E-04 & 0.07 & 1278 \\
\hline $241-\mathrm{AW}-102$ & 0.004 & 47.3 & $9.21 \mathrm{E}-05$ & 0.06 & $>1826$ \\
\hline $241-\mathrm{AW}-101$ & 0.001 & 7.1 & $8.26 \mathrm{E}-05$ & 0.06 & 238 \\
\hline 241-B-204 & 0.006 & 72.6 & $8.08 \mathrm{E}-05$ & 0.05 & 160 \\
\hline 241-B-203 & 0.006 & 79.4 & $7.39 \mathrm{E}-05$ & 0.05 & 145 \\
\hline 241-AN-101 & 0.002 & 42.6 & $5.51 \mathrm{E}-05$ & 0.04 & 364 \\
\hline
\end{tabular}

Note: ${ }^{1}$ Data from RPP-5926 Ammonia Evaluation.xls, SVF-1549.

${ }^{2}$ TTLFL data for hypothetical zero ventilation case from main text (i.e., Revision 8 of this document).

${ }^{3}$ 241-SY-101 ammonia liquid data taken before remediation by back-flush and dilution (PNNL-13933).

${ }^{4}$ 241-SY-102 high ammonia concentration measured soon after 241-SY-101 transfer. Previous maximum measured in 1997 was only $0.005 \mathrm{~kg}-\mathrm{mole} / \mathrm{m}^{3}$.

$\mathrm{NA}=$ Not Applicable, steady-state flammable gas concentrations remain low through tank dome diffusion.

Data in Table C-3 are sorted from highest to lowest theoretical ammonia concentration. Tanks with equilibrium ammonia concentrations greater than $2 \%$ of the LFL have long TTLFLs. Highest theoretical concentrations are estimated for SX Farm; however, steady-state flammable gas concentrations remain low for SX and AX tanks by gaseous molecular diffusion through the tank domes. Ammonia data for 241-S-102 predates retrieval, and current concentrations would be only a fraction of the concentration shown in Table C-3. Ammonia data for 241-SY-101 predates remediation by flush and dilution, and current concentrations would be significantly lower.

Tanks 241-S-102, 241-A-101, 241-BY-106 and 241-S-101 have TTLFLs longer than 5 years. Tanks 241-U-103 and 241-U-105 have calculated ammonia equilibrium concentrations of $5.1 \%$ 
of the LFL, but take more than 3 years to reach the LFL under hypothetical zero ventilation conditions. The tank with the shortest TTLFL with an equilibrium concentration higher than $2 \%$ of the LFL is 241-AN-103. However, it still takes a year to reach the LFL under hypothetical zero ventilation conditions, and has a calculated equilibrium ammonia concentration of only $2.4 \%$ of the LFL.

\section{C3.3 AMMONIA MASS TRANSFER}

This section examines limitations in reaching some of the peak concentrations shown in Table C-3.

\section{C.3.3.1 Molecular Diffusion Through Waste Liquid}

In the absence of mechanical agitation or convective movement, mass transfer is limited to molecular diffusion. Ammonia is produced predominantly in the waste liquid through degradation of organic complexants (RPP-21854). Ammonia transport is not possible through the tank shell, so there must be a bulk gradient within the non-convective waste layers with higher concentrations towards the tank bottom and lower concentrations towards the top.

It takes years for ammonia to molecularly diffuse through the liquid waste. This can be illustrated using tank 241-U-103. PNNL-13000 contains liquid ammonia measurements for several SST wastes. The data have considerable uncertainty and are heavily caveated but are sufficient to determine a time scale for molecular diffusion through non-convective waste layers. From PNNL-13000, ammonia concentration near the waste surface (segment 2) of tank $241-\mathrm{U}-103$ was about $0.0026 \mathrm{~kg}-\mathrm{mole} / \mathrm{m}^{3}$ and was about $0.084 \mathrm{~kg}-\mathrm{mole} / \mathrm{m}^{3} 57$ inches $(145 \mathrm{~cm})$ deeper at segment 5 .

Equation C-7 governs one-dimensional diffusion (assuming constant D, and negligible net flow):

Where

$$
\mathrm{D} \frac{\partial^{2} \mathrm{C}}{\partial \mathrm{x}^{2}}=\frac{\partial \mathrm{C}}{\partial \mathrm{t}}
$$

$$
\begin{aligned}
\mathrm{D} & =\text { diffusivity } \\
\mathrm{C} & =\text { concentration } \\
\mathrm{x} & =\text { length } \\
\mathrm{t} & =\text { time }
\end{aligned}
$$

Diffusion is analogous to flow of heat in a solid and is expressed by the same partial differential equation [Conduction of Heat in Solids (Carslaw and Jaeger 2003)] with a few appropriate term substitutions: 
Where

$$
K \frac{\partial^{2} v}{\partial \mathrm{x}^{2}}=\frac{\partial v}{\partial \mathrm{t}}
$$

$$
\begin{aligned}
K & =\text { thermal conductivity } \\
v & =\text { temperature } \\
\mathrm{x} & =\text { length } \\
\mathrm{t} & =\text { time }
\end{aligned}
$$

Figure C-2 shows the temperature profile in Tank 241-U-103. Heat is generated throughout the waste layer and temperature profiles indicate conduction through the waste solids (nonconvective layer). To simplify the analysis, temperature at the bottom is assumed to remain constant in the lower 100 inches of waste and the top of the waste layer is assumed to be an adiabatic boundary.

Figure C-2. Temperature Profile in Tank 241-U-103

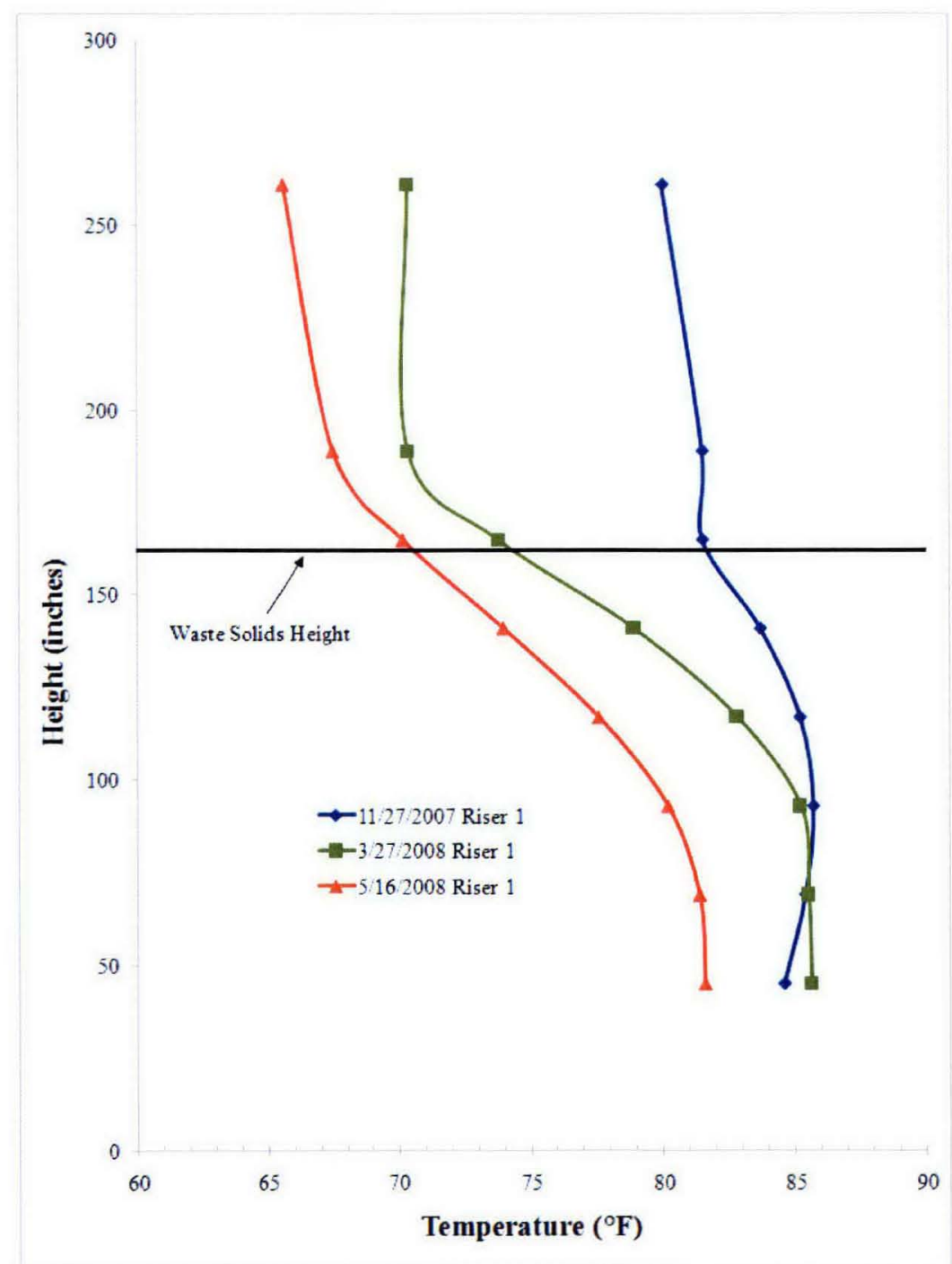




\section{RPP-5926 REV 9}

Ammonia is also generated throughout the waste liquid, and generation is most likely highest towards the bottom where temperature is highest. For this analysis, ammonia concentration is assumed to remain constant in the lower 100 inches (through continued slow organic degradation) of the waste and mass transfer out of the top of the waste layer is assumed to stop.

This is a simplification of the scenario, because ammonia production would continue wherever liquid waste resides (including the top 59 inches). However, ammonia generation and release are slow relative to hydrogen generation and release (see Section C3.3.3).

Diffusion is analogous to heat flow in a solid of thickness $l$ bounded by two parallel planes where one end is at a constant temperature and the other end is insulated (see Figure C-3).

Figure C-3. One-Dimensional Heat Conduction Analogy.

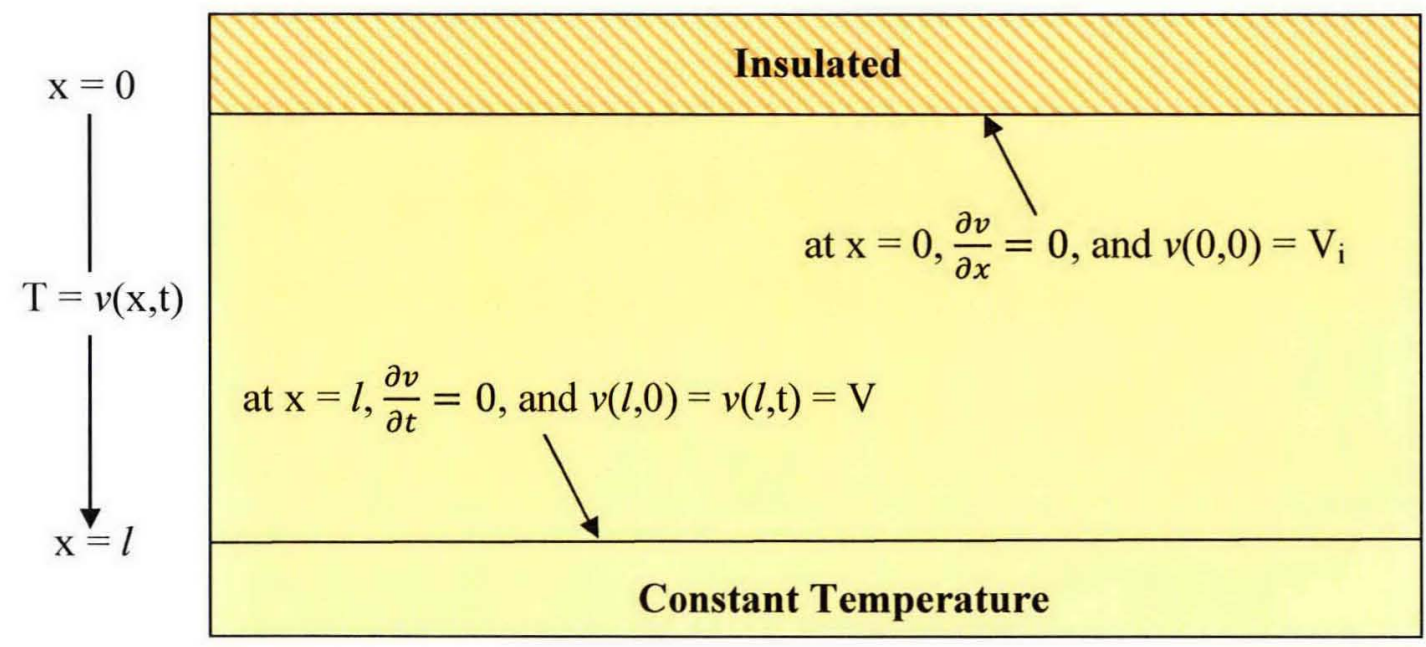

Carslaw and Jaeger (2003) give the solution shown in Equation C-9 for the boundary conditions where the end $\mathrm{x}=0$ is insulated and the end $\mathrm{x}=l$ is kept at constant temperature $\mathrm{V}$. The initial temperature in the slab is $f\left(x^{\prime}\right)$.

$v=V+\frac{2}{l} \sum_{\mathrm{n}=0}^{\infty} \mathrm{e}^{-\mathrm{K}(2 \mathrm{n}+1)^{2} \pi^{2} \mathrm{t} / 4 l^{2}} \cos \frac{(2 \mathrm{n}+1) \pi x}{2 l}\left\{\frac{2 l(-1)^{\mathrm{n}+1} \mathrm{~V}}{(2 \mathrm{n}+1) \pi}+\int_{0}^{l} f\left(x^{\prime}\right) \cos \frac{(2 \mathrm{n}+1) \pi x^{\prime}}{2 l} d x^{\prime}\right\}$

The initial ammonia concentration in the waste liquid is assumed to vary linearly from $\mathrm{V}$ at segment 5 (i.e., $\mathrm{x}=l$ ) to $\mathrm{V}_{\mathrm{i}}$ at segment 2 (i.e., at $\mathrm{x}=0$ ), thus

$$
f\left(x^{\prime}\right)=\frac{\left(\mathrm{V}-\mathrm{V}_{\mathrm{i}}\right)}{l} x^{\prime}+\mathrm{V}_{\mathrm{i}}
$$


and the integration interval becomes

$$
\frac{\left(\mathrm{V}-\mathrm{V}_{\mathrm{i}}\right)}{l} \int_{0}^{l} x^{\prime} \cos \frac{(2 n+1) \pi x^{\prime}}{2 l} d x^{\prime}+\mathrm{V}_{\mathrm{i}} \int_{0}^{l} \cos \frac{(2 n+1) \pi x^{\prime}}{2 l} d x^{\prime}
$$

Integrating results in

$$
\begin{aligned}
& \frac{\left(\mathrm{V}-\mathrm{V}_{\mathrm{i}}\right)}{l}\left|\frac{4 l^{2}}{[(2 n+1) \pi]^{2}} \cos \frac{(2 n+1) \pi x^{\prime}}{2 l}+\frac{2 l x}{(2 n+1) \pi} \sin \frac{(2 n+1) \pi x^{\prime}}{2 l}\right|_{0}^{l} \\
& +\mathrm{V}_{\mathrm{i}}\left|\frac{2 l}{(2 n+1) \pi} \sin \frac{(2 n+1) \pi x^{\prime}}{2 l}\right|_{0}^{1}
\end{aligned}
$$

Evaluating the integral, $\sin (0)=0, \cos (0)=1, \sin \frac{(2 n+1) \pi}{2}=(-1)^{n}$, and $\cos \frac{(2 n+1) \pi}{2}=0$, and Equation C-9 now becomes

$$
\begin{aligned}
& \mathrm{v}=\mathrm{V}+\frac{2}{l} \sum_{\mathrm{n}=0}^{\infty} \mathrm{e}^{-\mathrm{K}(2 \mathrm{n}+1)^{2} \pi^{2} \mathrm{t} / 4 l^{2}}\left[\cos \frac{(2 \mathrm{n}+1) \pi x}{2 l}\right]\left\{\frac{2 \mathrm{l}(-1)^{\mathrm{n}+1} \mathrm{~V}}{(2 \mathrm{n}+1) \pi}\right. \\
& \left.+\frac{\left(\mathrm{V}-\mathrm{V}_{\mathrm{i}}\right)}{l}\left[\frac{2 l^{2}(-1)^{\mathrm{n}}}{(2 \mathrm{n}+1) \pi}-\frac{4 l^{2}}{[(2 \mathrm{n}+1) \pi]^{2}}\right]+\mathrm{V}_{\mathrm{i}}\left[\frac{2 l(-1)^{n}}{(2 \mathrm{n}+1) \pi}\right]\right\}
\end{aligned}
$$

The point of interest is at the insulated end (i.e., where $\mathrm{x}=0$ ), thus the cosine term is always equal to 1 . With the cosine term equal to $1, l$ can be factored out and Equation $\mathrm{C}-10$ simplifies to

$$
\begin{aligned}
v=V+ & 4 \sum_{n=0}^{\infty} e^{-K(2 n+1)^{2} \pi^{2} t / 4 l^{2}}\left\{\frac{(-1)^{n+1} V}{(2 n+1) \pi}+\left(V-V_{i}\right)\left[\frac{(-1)^{n}}{(2 n+1) \pi}-\frac{2}{[(2 n+1) \pi]^{2}}\right]\right. \\
& \left.+V_{i}\left[\frac{(-1)^{n}}{(2 n+1) \pi}\right]\right\}
\end{aligned}
$$

Dividing both sides by $\mathrm{V}$, and recognizing that $\mathrm{C}=v, \mathrm{C}_{0}=\mathrm{V}, \mathrm{V}_{\mathrm{i}}=\mathrm{C}_{\mathrm{i}}$ and $\mathrm{K}=\mathrm{D}$; Equation C-11after simplification becomes

$$
\frac{C}{C_{0}}=1-8 \sum_{n=0}^{\infty} \frac{\left(1-C_{i} / C_{0}\right)}{(2 n+1) \pi} e^{-D(2 n+1)^{2} \pi^{2} t / 4 l^{2}}
$$


Table C-4 shows the liquid waste levels for tanks with hypothetical equilibrium ammonia headspace concentrations above $2 \%$ of the LFL (excluding SX and AX Tanks that allow substantial flammable gas diffusion through the domes). Tank 241-U-103 has the shallowest liquid height at 60 inches $(\sim 152 \mathrm{~cm})$. This tank will be used for illustration purposes because it has the shortest diffusion path length. Using Equation C-12 and the 241-U-103 data described earlier (i.e., $\mathrm{C}_{\mathrm{i}}=0.0026 \mathrm{~kg}$-moles $/ \mathrm{m}^{3}, \mathrm{C}_{0}=0.084 \mathrm{~kg}-\mathrm{moles} / \mathrm{m}^{3}$, and $l=145 \mathrm{~cm}$ ) and a diffusivity of $0.00002 \mathrm{~cm}^{2} / \mathrm{s}$ for ammonia in water (Perry's 1984) results in the plot shown in Figure C-4 (SVF-1560).

Table C-4. Waste Levels.*

\begin{tabular}{|c|c|c|}
\hline Tank & $\begin{array}{c}\text { Total Waste Level } \\
\text { (inches) }\end{array}$ & $\begin{array}{c}\text { Waste Liquid Level } \\
\text { (inches) }\end{array}$ \\
\hline $241-\mathrm{A}-101$ & 127 & $127 \dagger$ \\
\hline $241-\mathrm{S}-101$ & 137 & $125 \dagger$ \\
\hline $241-\mathrm{U}-105$ & 137 & $101 \dagger$ \\
\hline $241-\mathrm{BY}-106$ & 168 & $84 \dagger$ \\
\hline $241-\mathrm{S}-111$ & 153 & $99 \dagger$ \\
\hline $241-\mathrm{SY}-103 \dagger$ & $\sim 268$ & $\sim 268$ \\
\hline $241-\mathrm{U}-103$ & 162 & $60 \dagger$ \\
\hline $241-\mathrm{S}-103$ & 93 & $92 \dagger$ \\
\hline $241-\mathrm{U}-108$ & 152 & $116 \dagger$ \\
\hline $241-\mathrm{A}-103$ & 140 & $140 \dagger$ \\
\hline $241-\mathrm{U}-109$ & 155 & $78 \dagger$ \\
\hline $241-\mathrm{U}-106$ & 76 & $72 \dagger$ \\
\hline $241-\mathrm{U}-102$ & 138 & $82 \dagger$ \\
\hline $241-\mathrm{SY}-101$ & 401 & 401 \\
\hline $241-\mathrm{AN}-103 \dagger$ & $\sim 350$ & $\sim 348$ \\
\hline
\end{tabular}

Notes: * Data from PCSACS accessed 08/25/2008.

$\dagger$ Liquid level from neutron probe measurements.

$\$$ Tanks 241-SY-103 and 241-AN-103 waste levels fluctuate as buoyant displacement gas release events occur.

Examining Figure C-4, when mass transfer into the tank headspace is hypothetically stopped, ammonia concentration near the top of the liquid waste layer asymptotically approaches $\mathrm{C}_{0}($ the concentration at a depth of $145 \mathrm{~cm}$ ). After one year, concentration at the top boundary $(\mathrm{C})$ is only about $22 \%$ of $\mathrm{C}_{0}$ and is only $45 \%$ of $\mathrm{C}_{0}$ after five years. This helps explain the relatively low ammonia temporal variability observed in headspace measurements.

TTLFL results show that hydrogen and methane generation will bring headspace concentration to the LFL in the bounding tank (241-AZ-102) in about 52 days. From Table C-3, 14 tank headspaces reach the LFL in a year or less, with only 241-AN-104 having a calculated equilibrium ammonia concentration more than $2 \%$ of the LFL. Because the TTLFL interval of interest ranges from weeks to months, using maximum equilibrium ammonia concentrations in TTLFL calculations results in gross over-conservatism. 


\section{RPP-5926 REV 9}

Figure C-4. Ammonia Concentration Change as a Function of Time

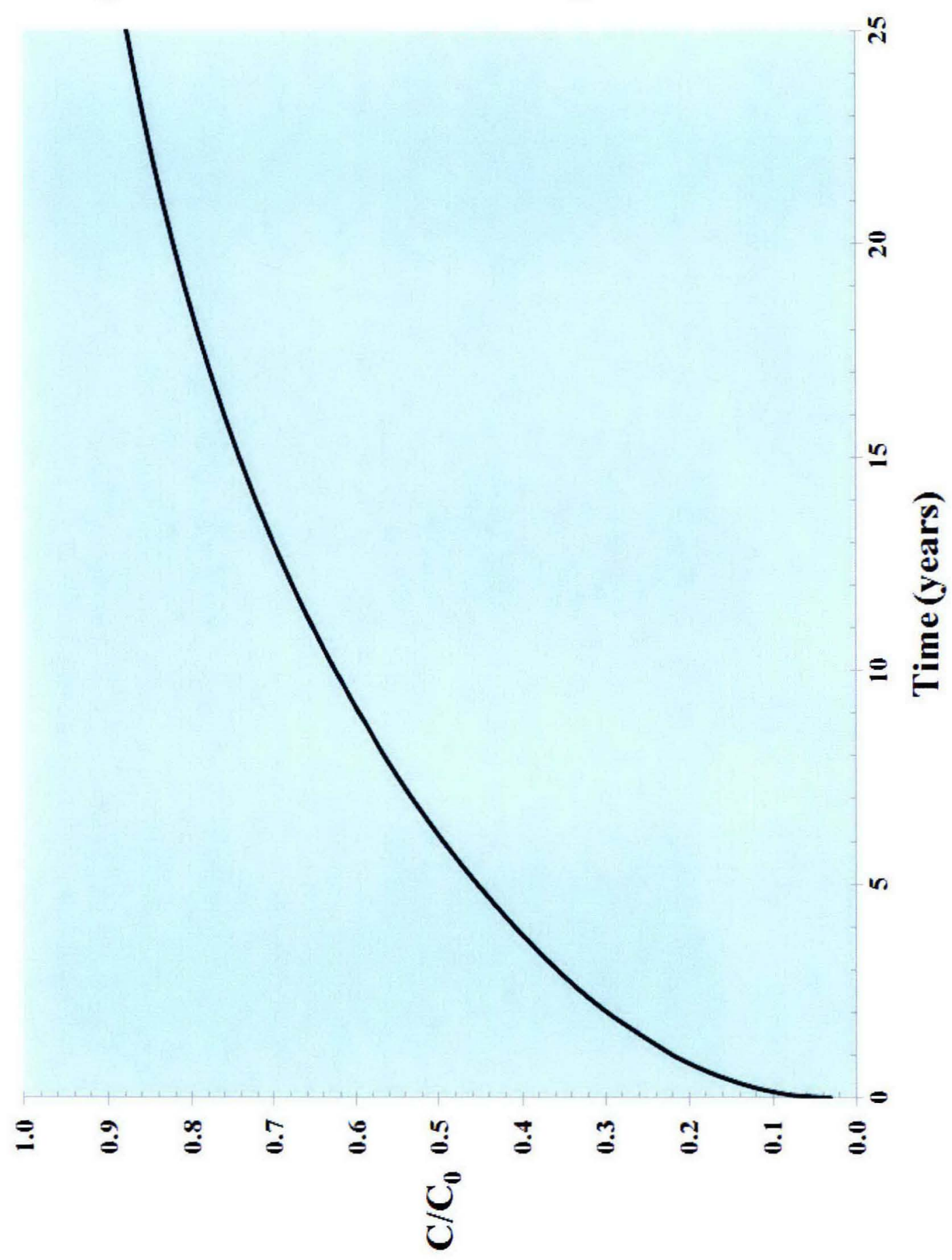




\section{C3.3.2 Natural Convection}

Waste configurations differ significantly between DSTs and SSTs. While SSTs have no or negligible supernatant layers, most DSTs have some waste solids at the bottom covered by a significant liquid waste layer. This leads to a temperature gradient between the bottom and top as observed in the two thermocouple trees for tank 241-SY-103 (Figure C-5). A simple check shows that natural convective heat transfer through the liquid waste layer exceeds conduction by several orders of magnitude.

The Nusselt number $(\mathrm{Nu})$ is the ratio of convective to conductive heat transfer and can be calculated for a characteristic length. The average Nusselt number can be related to the Grashof and Prandtl numbers by an equation of the form (Kreith and Black 1980)

Where

$$
\overline{\mathrm{Nu}_{f}}=\mathrm{C}\left(\mathrm{Gr}_{f} \mathrm{Pr}_{f}\right)^{\mathrm{n}}
$$

$$
\begin{aligned}
f & =\text { subscript indicating physical properties at the average temperature } \\
\mathrm{C} & =\text { empirical constant } \\
\mathrm{n} & =\text { empirical exponential term }
\end{aligned}
$$

The Grashof number is given by (Kreith and Black 1980)

Where

$$
\mathrm{Gr}=\frac{\beta g \rho^{2}\left(\mathrm{~T}_{\mathrm{s}}-\mathrm{T}_{\infty}\right) \mathrm{L}^{3}}{\mu^{2}}
$$

$$
\begin{aligned}
\beta & =\text { volumetric thermal expansion coefficient, } \mathrm{K}^{-1} \\
\mathrm{~g} & =\text { gravitational constant, } 9.8 \mathrm{~m} / \mathrm{s}^{2} \\
\rho & =\text { density, } \mathrm{kg} / \mathrm{m}^{3} \\
\mathrm{~T}_{\mathrm{S}}-\mathrm{T}_{\infty} & =\text { temperature difference, } \mathrm{K} \\
\mathrm{L} & =\text { characteristic length, } \mathrm{m} \\
\mu & =\text { viscosity, } \mathrm{Pa}-\mathrm{s}(\mathrm{kg} / \mathrm{m}-\mathrm{s})
\end{aligned}
$$


Figure C-5. Temperature Profile in Tank 241-SY-103
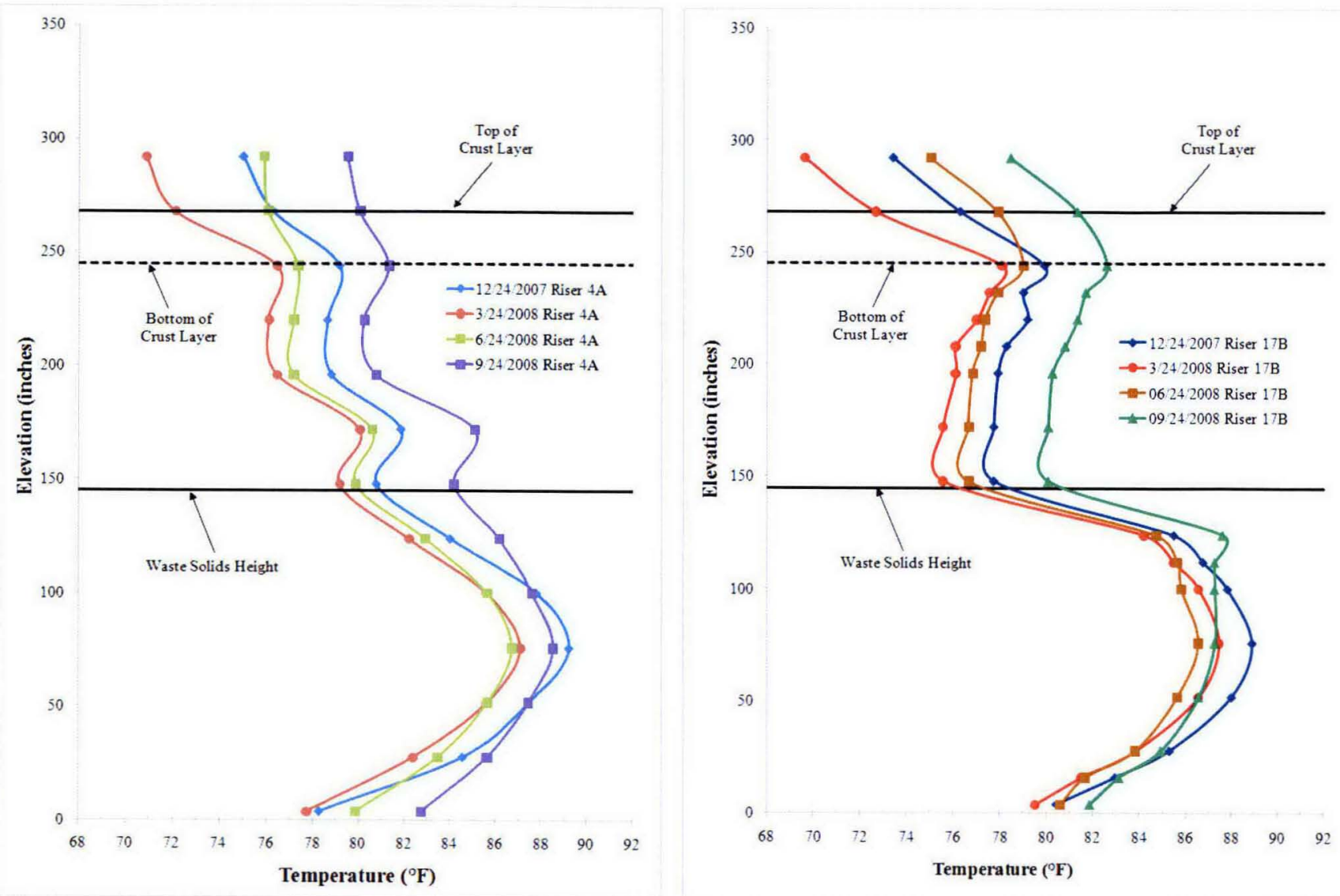

The Prandtl number is given by (Kreith and Black 1980)

$$
\operatorname{Pr}=\frac{C_{\mathrm{P}} \mu}{K}
$$

Where

$\mathrm{C}_{\mathrm{P}}=$ specific heat, $\mathrm{j} / \mathrm{kg}-\mathrm{K}$

$K=$ thermal conductivity, watt $/ \mathrm{m}-\mathrm{K}$

The average temperature within the convective layer is about $80^{\circ} \mathrm{F}(300 \mathrm{~K})$. Specific heat for water at $300 \mathrm{~K}$ is about $4180 \mathrm{j} / \mathrm{kg}-\mathrm{K}$ (CRC 1980). Viscosity for the saturated liquid waste was estimated to be 1 to $10 \mathrm{cp}$ (PNNL-11296). Thermal conductivity of water at $300 \mathrm{~K}$ is about 0.609 watt $/ \mathrm{m}-\mathrm{K}$ (CRC 1980). The Prandtl number is

$$
\operatorname{Pr}=\frac{\left(4180 \frac{\mathrm{j}}{\mathrm{kg}-\mathrm{K}}\right)\left(0.005 \frac{\mathrm{kg}}{\mathrm{m}-\mathrm{s}}\right)}{\left(0.609 \frac{\mathrm{j}}{\mathrm{s}-\mathrm{m}-\mathrm{K}}\right)} \approx 34
$$


The volumetric thermal expansion coefficient for water at $300 \mathrm{~K}$ is $0.0002761 \mathrm{~K}^{-1}$ (CRC 1980). Density of the 241-SY-103 convective layer is about $1490 \mathrm{~kg} / \mathrm{m}^{3}$ (TWINS 2008). From

Figure C-5, there is about a $7^{\circ} \mathrm{F}(4 \mathrm{~K})$ temperature drop between the waste solids layer and the crust layer. The length from the top of the waste solids to the bottom of the crust layer is about 123 inches $(3.12 \mathrm{~m})$. Using these values, the Grashof number is

$$
\mathrm{Gr}=\frac{(0.0002761)(9.8)(1490)^{2}(4)(3.12)^{3}}{(0.005)^{2}} \approx 2.92 \times 10^{10}
$$

The product GrPr is $9.9 \times 10^{11}$. For a geometry of a lower warm plate and an upper cool plate, $\mathrm{C}$ is 0.58 and $n=0.2$ (Kreith and Black 1980). The Nusselt number is then

$$
\overline{\mathrm{Nu}_{f}}=0.58\left(1.0 \times 10^{12}\right)^{0.2} \approx 145
$$

Heat transfer through convection is about two orders of magnitude higher than conductive heat transfer suggesting the convective layer is well mixed. This is corroborated by other studies that examined DSTs (e.g., RPP-16841) and the temperature measurements which are fairly uniform through the liquid waste layer.

Convective mixing coupled with active ventilation results in the relatively low ammonia partial pressures shown in Table C-3 for DST wastes. Considerable time would be required to bring liquid ammonia concentrations up to the levels necessary to exert an ammonia partial pressure that contributed $2 \%$ to the LFL. For example, from Table C-1 the headspace concentration in the convectively mixed tank 241-AP-104 was 260 ppm in November 2000. Ventilation through this tank was maybe as high as $150 \mathrm{ft}^{3} / \mathrm{min}$ and there were approximately 403 inches of waste. Assuming steady-state had been achieved, the ammonia generation rate to maintain this steadystate would have been approximately

$$
\frac{\left(150 \frac{\mathrm{ft}^{3}}{\min }\right)\left(\frac{1 \mathrm{~min}}{60 \mathrm{~s}}\right)\left(\frac{1 \mathrm{~m}^{3}}{35.3 \mathrm{ft}^{3}}\right)\left(\frac{0.00026 \mathrm{~m}^{3} \text { of } \mathrm{NH}_{3}}{1 \mathrm{~m}^{3}}\right)\left(\frac{1 \mathrm{~kg}-\mathrm{mole} \mathrm{of} \mathrm{NH}_{3}}{22.4 \mathrm{~m}^{3} \mathrm{of} \mathrm{NH}_{3}}\right)}{(403 \text { inches })\left(\frac{2750 \text { gallons }}{1 \text { inch }}\right)\left(\frac{1 \mathrm{~m}^{3}}{264 \text { gallons }}\right)} \approx 1.96 \times 10^{-10} \frac{\mathrm{kg}-\mathrm{moles} \text { of } \mathrm{NH}_{3}}{\mathrm{~m}^{3}-\mathrm{s}}
$$

Using the data from Table C-3 and the estimated ammonia generation rate, it is possible to calculate how long it would take for ammonia to contribute $2 \%$ of the LFL (i.e., reach a partial pressure of $0.003 \mathrm{~atm}$ in the headspace) assuming zero ventilation. From Equation C-3, ammonia liquid waste concentration would need to increase from $0.008 \mathrm{~kg}-\mathrm{mole} / \mathrm{m}^{3}$ to

$$
\left(40.0 \frac{\mathrm{kg}-\mathrm{mole}}{\mathrm{m}^{3}-\mathrm{atm}}\right)(0.003 \mathrm{~atm})=0.120 \frac{\mathrm{kg}-\mathrm{mole}}{\mathrm{m}^{3}} .
$$

Total waste volume in 241-AP-104 was about $4198 \mathrm{~m}^{3}$ in November 2000 . The kg-moles of ammonia accumulated in the waste would need to be

$$
\left(0.120 \frac{\mathrm{kg}-\mathrm{mole}}{\mathrm{m}^{3}}-0.008 \frac{\mathrm{kg}-\mathrm{mole}}{\mathrm{m}^{3}}\right)\left(4198 \mathrm{~m}^{3}\right) \approx 470 \mathrm{~kg}-\text { moles }
$$


The time required to generate the ammonia to reach a contribution of $2 \% \mathrm{LFL}$ would be

$$
\frac{470 \mathrm{~kg}-\text { moles }}{\left(1.96 \times 10^{-10} \frac{\mathrm{kg}-\mathrm{moles}_{\mathrm{NH}}}{\mathrm{m}^{3} \mathrm{~s}}\right)\left(4198 \mathrm{~m}^{3}\right)} \approx 5.7 \times 10^{8} \text { s or } 18 \text { years. }
$$

Even if the ammonia generation rate in 241-AP-104 were an order of magnitude higher, it would still take close to two years for ammonia to contribute $2 \%$ of the LFL.

The only convectively mixed tanks with relatively high ammonia partial pressures are (in decreasing order) 241-SY-103, 241-SY-101, 241-AN-103 and 241-AN-104. Examining these tanks more closely, the reason for these exceptions becomes apparent; all have thick contiguous floating crust layers $(23,35,35$ and 16 inches, respectively). Tank 241-SY-101 had a floating crust layer when the liquid ammonia concentration was measured, but has since been remediated.

Convective mixing keeps temperatures and ammonia concentrations fairly uniform through the waste liquid, however, temperature trends show a linear decrease through the crust layer. Although mass transfer through the liquid layer would be relatively fast, the floating crust provides a barrier to convective mass transfer. About $96 \%$ of the crust (RPP-6655) is below the liquid surface so heat (and mass) transfer through this region is by conduction (diffusion for mass).

Crusts are a mixture of solids, liquid and gas. Ammonia must diffuse through the connected porosity which would have to be considerably more tortuous than the simple diffusion presented in Section C3.3.1. Effective diffusivity through the brine laden crust (or non-convective waste for that matter) would likely be more than an order of magnitude lower than the diffusivity of ammonia through water. It would take years for headspace concentrations to reach equilibrium concentrations in the tanks with floating crust layers.

\section{C3.3.3 Mechanical Mixing}

Mechanical mixing does occur to a limited extent, with the most significant mechanism being buoyant displacement gas release events (BDGREs) in a few DSTs. BDGREs occur in tanks 241-SY-103, 241-AN-103, 241-AN-104, 241-AN-105 and 241-AW-101. In a BDGRE, a portion, or "gob," of the non-convective layer accumulates gas until it becomes sufficiently buoyant to overcome the weight and strength of material restraining it. At that point, it suddenly breaks away and rises through the convective layer. The trapped gas bubbles expand as the gob rises, failing the surrounding material and disturbing the crust layer, so a portion of the gas can escape from the gob into the headspace.

BDGREs mechanically mix a portion of the crust layer; however, they occur infrequently (currently about every six months for 241-SY-103) and are relatively small (releasing just a few cubic meters of gas). Before remediation, Tank 241-SY-101 use to have vigorous BDGREs that would involve six to eight gobs that significantly mixed the crust layer. BDGREs in the other tanks disturb only a fraction of the floating crust layer and are short duration lasting hours to a couple of days. Therefore, BDGREs would not significantly decrease the time required to reach ammonia equilibrium concentration. 
Tanks not subject to BDGREs also generate non-condensable gasses (predominately hydrogen), but the gas percolates through waste solids at about the same rate it is generated. Gas percolation could slightly increase mixing through liquid saturated solid layers, but gas generation is slow and this effect would be small.

Some DSTs (e.g., 241-AY-102) have had caustic additions which might increase supernatant densities above that of the solid's interstitial liquid. Analysis (RPP-29464) suggests that such density gradients might lead to porous media convection. Ammonia is retained in the waste liquid the bulk of which is supernatant in DSTs. Porous media convection would have a negligible effect on ammonia release from DST wastes.

\section{C4.0 ANALYSIS}

Data in Section C.3.1.1 show that ammonia headspace concentrations under normal operating conditions are low with only 3 of the 127 tanks measured reaching more than $1 \%$ of the LFL, and all were below $2 \%$ of the LFL. Statistical analysis of ammonia headspace data indicate that concentrations are only modestly influenced by the slowly changing tank conditions.

Only during waste disturbing activities discussed in Section C.3.1.2 did headspace concentrations exceed $2 \%$ of the LFL, and then only in a limited number of tanks that contained high liquid ammonia concentrations. Elevated ammonia concentrations during waste disturbing activities are only temporary and relatively short compared to calculated TTLFLs and the mass transport limitations discussed in Section C3.3.

Liquid ammonia concentrations can be considerable in the wastes, and calculations performed in Section C3.2.3 show that hypothetical headspace maximums might be significant. Once tanks that have low flammability ensured by gaseous diffusion through tank domes (i.e., the AX and SX tanks) and 241-S-102 and 241-SY-101 are eliminated, then only 16 tanks had theoretical maximum concentrations greater than $2 \%$ of the LFL. Analyses in Section C3.3 suggest limits on how quickly maximum concentration could be reached. Molecular diffusion requires years for ammonia to move through non-convective waste layers.

Most DST liquid wastes exert low ammonia vapor pressures because mass transport through the convective layers is relatively fast. If ventilation were stopped, it would take many years to accumulate sufficient ammonia in the waste liquid to significantly increase headspace flammability. DSTs with ammonia equilibrium vapor pressures greater than $2 \%$ of the LFL all have floating crust layers. Although convective mass transfer through the liquid layer is fast, floating crusts provide a barrier to convection. Ammonia must diffuse through the connected porosity within the floating crusts. Time spans to reach these higher equilibrium concentrations are beyond the weeks to months of interest in TTLFL calculations. The use of maximum equilibrium ammonia concentrations in TTLFL calculations results in gross over-conservatism. 
A reasonably conservative ammonia flammability contribution would be a value slightly higher than the maximum concentration measured under quiescent conditions. The maximum measured under quiescent conditions was $1.67 \%$ of the LFL in Tank 241-U-103. Starting TTLFL calculations at $2 \%$ of the LFL would be a reasonably conservative representation of ammonia flammability. This remains a reasonably conservative assumption even as wastes are moved from SSTs to DSTs and among the DSTs. Interim stabilization already moved most of the high ammonia producing liquid waste into the DSTs and ammonia generation should continue to decrease as wastes are mixed and decay power decreases.

\section{C5.0 CONCLUSIONS}

The previous ammonia flammability methodology required steady-state ammonia concentrations, headspace ventilation rates, and ammonia liquid concentrations to be known simultaneously for current conditions. These data are not routinely collected and the available data in some cases do not accurately reflect current waste tank conditions. This appendix demonstrates that a reasonably conservative single value can be added to TTLFL calculations to account for ammonia contribution to total flammability. Starting TTLFL calculations at $2 \%$ of the LFL is a reasonably conservative representation of ammonia contribution to flammability.

\section{C6.0 REFERENCES}

Carslaw, H. S., and J. C. Jaeger, 2003, Conduction of Heat in Solids, Second Edition, Oxford University Press, Oxford, United Kingdom.

Clegg, S. L., and P. Brimblecombe, 1989, Journal of Physical Chemistry, Vol. 93, pg. 7237.

CRC, 1980, Handbook of Chemistry and Physics, $61^{\text {st }}$ Edition, CRC Press, Inc., Boca Raton, Florida.

Kreith, F. and W. Z. Black, 1980, Basic Heat Transfer, Harper \& Row, Publishers, Inc., New York, New York.

Perry, R. H. and D. W. Green, 1984, Perry's Chemical Engineers' Handbook, Sixth Edition, McGraw-Hill Book Company, New York, New York.

PNNL-13000, 1999, Retained Gas Sampling Results for the Flammable Gas Program, Rev. 0, Pacific Northwest National Laboratory, Richland, Washington.

PNNL-13933, 2002, Review of the Technical Basis of the Hydrogen Control Limit for Operations in Hanford Tank Farms, Rev. 1, Pacific Northwest National Laboratory, Richland, Washington.

RPP-4941, 2000, Methodology for Predicting Flammable Gas Mixtures in Double-Contained Receiver Tanks, Rev. 0-B, CH2M HILL Hanford Group, Inc., Richland, Washington. 


\section{RPP-5926 REV 9}

RPP-6655, 2000, Data Observations on Double-Shell Flammable Gas Watch List Tank

Behavior, Rev. 0, CH2MHILL Hanford Group, Inc., Richland, Washington.

RPP-7771, 2001, Flammable Gas Safety Issue Resolution, Rev. 0A, CH2M HILL Hanford Group, Inc., Richland, Washington.

RPP-16841, 2003, Engineering Evaluation of 241-C-106 Waste Transfer into Tank 241-AN-106, Rev. 0, CH2M HILL Hanford Group, Inc., Richland, Washington.

RPP-21854, 2004, Occurrence and Chemistry of Organic Compounds in Hanford Site Waste Tanks, Rev. 0, CH2M HILL Hanford Group, Inc., Richland, Washington.

RPP-21972, 2006, Statistical Analysis of Tank Headspace Vapor Data, Rev. 1, CH2M HILL Hanford Group, Inc., Richland, Washington.

RPP-29464, 2006, Caustic Dynamic Mixing Analysis for Tank 241-AY-102, Rev. 0, CH2M HILL Hanford Group, Inc., Richland, Washington.

SVF-1549, 2008, RPP-5926 Ammonia Evaluation, v1.0, Washington River Protection Solutions, Richland, Washington.

SVF-1560, 2008, RPP-5926 Figure C-4, v1.0, Washington River Protection Solutions, Richland, Washington.

Tank Waste Information Network (TWINS), Queried 08/14/2008, http://twins.pnl.gov/twins.htm.

Weisenberger, S., and A. Schumpe, 1996, "The Estimation of Gas Solubilities in Salt Solutions at Temperature from $273 \mathrm{~K}$ to $363 \mathrm{~K}$," AIChE Journal, Vol. 42, pg. 299. 
RPP-5926 REV 9

\section{APPENDIX D}

\section{EVALUATION OF SST TEMPERATURE TRENDS}

D-1 


\section{D1.0 INTRODUCTION}

Periodic monitoring of DST temperatures will be conducted to ensure that transfers or storage conditions have not resulted in unanticipated increases in DST temperatures. The following discussion evaluates temperature trends in the SSTs to determine whether monitoring is required to ensure that temperatures do not increase over current levels.

HNF-3588, Organic Complexant Topical Report, discussed SST temperature trends as a means to evaluate the potential for runaway chemical reactions. Because of changes in the status of a number of SSTs (all tanks are now passively ventilated, and the high-heat sludge in tank 241-C-106 has been removed), the evaluation must be updated.

\section{D2.0 HNF-3588 EVALUATION}

Section 3.3.4.1 of HNF-3588 discusses temperatures in the passively ventilated SSTs (at the time of evaluation). The analysis states that

"... temperatures in the tank waste will continue to decline, even as the waste slowly dries and the thermal conductivity decreases. This is true even if the tank is interim stabilized.

The conclusion that the time-averaged annual temperatures in the passively ventilated tanks are continuing to decline is also corroborated by the tank temperature trends over the past fifteen years. The historical temperature data show that the time-averaged temperatures within the solid waste are slowly decreasing ... Examination of the tank headspace temperatures also indicate waste temperatures and heat loads are decreasing ... Waste temperatures after interim stabilization have also continued to decline."

\section{D3.0 ADDITIONAL EVALUATION FOR TANK 241-C-106, 241-A-101, AND 241-SX TANKS}

No radioactive waste has been added to the SSTs since 1980. The major radioactive heat sources in the SSTs are Cs-137and Sr-90. These nuclides have half-lives of approximately 30 years, so the heat load in the SSTs has decreased by approximately half since 1980 .

Tanks that have had waste removed via saltwell pumping or retrieval have even lower heat loads. Saltwell pumping removed liquid from a number of SSTs and also decreased the overall heat load (Cs-137 is typically soluble). Saltwell pumping activities were completed by 2003. Retrieval activities have removed waste from a number of SSTs, including 241-C-106, which had been a high heat load tank, but now has a small residual waste heel of about $3 \mathrm{kgal}$.

Tank temperature and active ventilation (exhauster) history is available in the Surveillance Analysis Computer System (SACS) (WRPS 2009). The exhausters in SX Farm were run intermittently for many years, then turned off in 2004 (infrequent filter checks continued for two more years). 
Tank 241-C-106 has undergone retrieval activities since 1999, including sluicing and oxalic acid dissolution. The tank currently contains $3 \mathrm{kgal}$ of waste (HNF-EP-0182, Waste Tank Summary Report for Month Ending January 31, 2009). None of the tank thermocouples are in the small remaining heel of waste. Temperatures inside the tank vapor space vary seasonally in accordance with ambient temperature changes. There are no concerns with increasing tank temperatures with the small heel of waste remaining (approximately 1 inch if distributed evenly across the tank).

Tank 241-A-101 warranted a special discussion in HNF-3588 because although the bulk temperature was decreasing, the temperature in the floating crust and dome space increased slightly, possibly due to changing heat transfer conditions in the crust as a result of trapped gas. Since then, saltwell pumping was performed, removing the liquid layer below the crust and some of the interstitial liquid. The tank level dropped by over 200 inches as a result of saltwell pumping in the $2002-2003$ timeframe. Since then, the peak temperature in the waste dropped from $128^{\circ} \mathrm{F}$ (late 2003) to $109^{\circ} \mathrm{F}$ (August 2009).

Temperature data in SACS (WRPS 2009) indicate that many of the SX Farm tanks exhibit a decreasing temperature trend since 2004 when the exhausters were last operated extensively. The SX North exhauster was run an additional 12 times through 2006, but for periods of only 3 hours or less (for filter testing). The maximum temperature currently in SX farm is less than $190^{\circ} \mathrm{F}$. High temperatures since 2004 range from $82^{\circ} \mathrm{F}$ for $241-\mathrm{SX}-113$ to $190^{\circ} \mathrm{F}$ for 241-SX-111. Tanks 241-SX-102, $-103,-105,-107,-108$, and -114 have a decreasing trend since 2004. A number of other tanks (241-SX-101, -104, -106, -110, -111, -112, and -113) have a flat temperature trend with no discernible increase or decrease. Tank 241-SX-115 does not have temperature readings, but contains only $4 \mathrm{kgal}$ of waste (HNF-EP-0182). Tank 241-SX-109 shows a decreasing trend up until 2004, but an increasing trend from 2004 to about 2007, and a flat trend since then. Additional evaluation is required for this tank.

One of the two SX Farm exhausters was connected to tank 241-SX-109. Air flow from tanks 241-SX-101 through 241-SX-106 passed through 241-SX-109 (RPP-5515, Leak Assessment for Tanks 241-SX-102 and 241-SX-109). Examining the exhauster operating history (WRPS 2009) shows that the SX North exhauster (ventilating 241-SX-109) was operated for only 30 days from January 2000 to March 2003. From March 2003 until March 2004, the exhauster was operated for 273 out of 379 days ( $72 \%$ of the time). After 2004, the exhauster ran for just over 1 day of total run time in the next couple of years (for filter testing). It is reasonable to conclude that the increase in 241-SX-109 temperatures (after the exhauster operation was curtailed) is due to equilibration to passive ventilation conditions. The cooling effect of forced ventilation was magnified in this tank because the exhauster was attached directly to 241-SX-109. The temperature trend since 2007 is flat for $241-\mathrm{SX}-109$. 


\section{D4.0 CONCLUSION}

The HNF-3588 evaluation remains valid for the SSTs with the exception of 241-A-101, 241-C-106 and the SX Farm tanks. Upon reevaluation in 2009, tank 241-A-101 now exhibits a decreasing temperature trend at all elevations in the waste.

The high-heat generating waste in tank $241-\mathrm{C}-106$ was removed by retrieval into the DST system. The tank contains only $3 \mathrm{kgal}$ of waste (HNF-EP-0182). There are no concerns about temperature increases in the waste of this tank.

SX Farm tanks were actively exhausted periodically up until 2004. The maximum temperature currently in SX farm is less than $190^{\circ} \mathrm{F}$. If the tank temperatures were above the boiling point, significant interstitial water loss could be occurring, changing the thermal properties of the waste. Since the active ventilation was turned off, the SX tank temperature trends are either flat or declining, except for tank 241-SX-109. This tank exhibited a temperature increase from 2004 to 2007, which is considered to be equilibration following extensive exhauster operation in the 12 months prior to curtailing exhauster operation. Since 2007, the temperature trend is flat, indicating that temperatures have equilibrated to the passive ventilation conditions.

With the updated evaluation, all SSTs are now passively ventilated (except that active ventilation may be used to support some retrieval activities). All SSTs have flat or decreasing temperature trends. Temperature increases are not possible without significant changes to the waste. Any waste addition or significant addition of water and chemicals (e.g., retrieval activities) will be evaluated via HNF-SD-WM-OCD-015 (Tank Farm Waste Transfer Compatibility Program).

\section{D5.0 REFERENCES}

HNF-3588, 2003, Organic Complexant Topical Report, Rev. 1, CH2M HILL Hanford Group, Inc., Richland, Washington.

HNF-EP-0182, 2009, Waste Tank Summary Report for Month Ending January 31, 2009, Rev. 250, Washington River Protection Solutions, Richland, Washington.

HNF-SD-WM-OCD-015, 2009, Tank Farm Waste Transfer Compatibility Program, Rev. 18G, Washington River Protection Solutions, Richland, Washington.

RPP-5515, 1999, Leak Assessment for Tanks 241-SX-102 and 241-SX-109, Rev. 0, Lockheed Martin Hanford Corporation, Richland, Washington.

Surveillance Analysis Computer System (SACS), HISI ID No. 242, accessed August 11, 2009 ["Discrete," "SX Vent" (SX exhausters)]; ["Temperature," "241-SX-xxx"], http://www7.rl.gov/hisiconsol/Form/AppUpdate.cfm?AppID=242\&WasSearch=Y. 
RPP-5926 REV 9

APPENDIX E

PEER REVIEW CHECKLIST

E-1 


\section{Calculation Review Checklist}

Calculation Reviewed: RPP-5926 Revision 9

Scope of Review: Entire document

(e.g., document section or portion of calculation)

Engineer/Analyst: _. E. Meacham

Organizational Manager:

N.W. Kirch

nWkinl

Date

$10 / 26 / 2009$

Date: $10 / 26 / 2009$

This document consists of $\underline{93}$ pages and no attachments:

Yes No NA*

[X] [ ] [ ] 1. Analytical and technical approaches and results are reasonable and appropriate.

[X] [ ] [ ] 2. Necessary assumptions are reasonable, explicitly stated, and supported.

[X] [ ] [ ] 3. Ensure calculations that use software include a paper printout, microfiche, CDROM, or other electronic file of the input data and identification to the computer codes and versions used, or provide alternate documentation to uniquely and clearly identify the exact coding and execution process.

[X] [ ] [ ] 4. Input data were checked for consistency with original source information.

[ ] [ ] [X] 5. Key input data (e.g., dimensions, performance characteristics) that may affect equipment design is identified.

[X] [ ] [ ] 6. For both qualitative and quantitative data, uncertainties are recognized and discussed and the data is presented in a manner to minimize design interpretations.

[X] [ ] [ ] 7. Mathematical derivations were checked, including dimensional consistency of results.

[X] [ ] [ ] 8. Calculations are sufficiently detailed such that a technically qualified person can understand the analysis without requiring outside information.

[X] [ ] [ ] 9. Software verification and validation are addressed adequately.

[X] [ ] [ ] 10. Limits/criteria/guidelines applied to the analysis results are appropriate and referenced. Limits/criteria/guidelines were checked against references.

[X] [ ] [ ] 11. Conclusions are consistent with analytical results and applicable limits.

[X] [ ] [ ] 12. Results and conclusions address all points in the purpose.

[X] [ ] [ ] 13. Referenced documents are retrievable or otherwise available.

[X] [ ] [ ] 14. The version or revision of each reference is cited.

[X] [ ] [ ] 15. The document was prepared in accordance with TFC-ENG-DESIGN-C-10 Attachment A, "Calculation Format and Preparation Instructions."

[X] [ ] [ ] 16. Impacts on requirements have been assessed and change documentation initiated to incorporate revisions to affected documents, as appropriate.

[X] [ ] [ ] 17. All checker comments have been dispositioned and the design media matches the calculations.

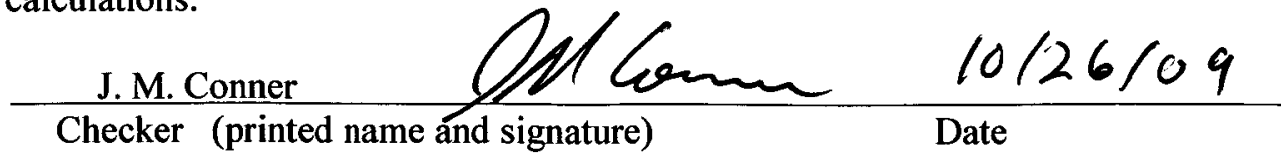

5. Calculations do not involve equipment design. 